Universidade de São Paulo

Instituto de Física

\title{
Mudança de Opinião em Redes Complexas: Aproximação de Campo Médio para o Modelo Sznajd
}

\author{
Maycon de Sousa Araújo \\ Orientadora: Profa. Dra. Carmen Pimentel Cintra do Prado
}

Dissertação apresentada ao Instituto de Física para a obtenção do título de Mestre em Ciências.

Banca Examinadora:

Profa. Dra. Carmen Pimentel Cintra do Prado (IF-USP)

Profa. Dra. Tânia Tomé Martins de Castro (IF-USP)

Prof. Dr. José Roberto Iglesias (IF-UFRGS)

São Paulo 


\section{FICHA CATALOGRÁFICA}

Preparada pelo Serviço de Biblioteca e Informação do Instituto de Física da Universidade de São Paulo

Araújo, Maycon de Sousa

Mudança de opinião em redes complexas: aproximação de campo médio para o modelo Sznajd. - São Paulo, 2011.

Dissertação (Mestrado) - Universidade de São Paulo. Instituto de Física - Depto. de Física Geral

Orientador: Profa. Dra. Carmen Pimentel Cintra do Prado

Área de Concentração: Física

Unitermos: 1. Mecânica Estatística; 2. Física Computacional; 3.Física da Matéria Condensada. 
À minha mãe, Maria Amélia de Sousa Araújo. À minha esposa, Francisca Maria Alves Silva. In memoriam, Raimundo Pereira de Araújo. 
"Toda a nossa ciência, comparada com a realidade, é primitiva e infantil, e, no entanto, é a coisa mais preciosa que temos."

Albert Einstein 


\section{Agradecimentos}

A realização deste trabalho foi conseguida com a generosidade, companheirismo e boa vontade de muitos. Agradeço a todos que contribuíram para sua concretização, em especial:

À professora Carmen Prado pela orientação e liderança do grupo.

Aos professores Sílvio Salinas, Marina Nielsen, Josif Frenkel, Émerson Passos, Nestor Caticha e Alberto Petri pelos cursos ministrados.

Ao amigo e colega de sala, Antônio Mario Ramos, e ao estudante de doutorado David Rodrigues pelas sugestões e discussões.

Ao estudante de doutorado André Timpanaro por sua importante colaboração no desenvolvimento computacional do trabalho.

Ao Departamento de Física Geral pelas condições de trabalho.

Ao Conselho Nacional de Desenvolvimento Científico e Tecnológico e a Universidade de São Paulo pelo suporte financeiro deste trabalho.

À minha mãe, Maria Amélia Araújo, e ao meu irmão, Benedito Silvestre Neto, pelo apoio, compreenção e incentivo em todos os momentos.

Ao meu pai, Raimundo Araújo, in memoriam.

À minha esposa, Francisca Maria Silva, pelos momentos de ternura, sacrifícios e superação.

Aos meus amigos, em particular, a Glauco Campêlo e sua família.

À sra. Maria Helena e sua família, aos quais tenho muito carinho e admiração. Por me estenderem a mão em momentos difíceis. Entre 
seus familiares, menciono destacadamente os meus agradecimentos à Marina Nóbrega. 


\section{Resumo}

Esta dissertação discutirá, com uma abordagem predominantemente analítica, aspectos em aberto do Modelo Sznajd e de algumas de suas variantes. Apresentaremos uma equação mestra que descreve a evolução de opiniões para o modelo e estudaremos seus estados estacionários numa aproximação de campo médio. Mostraremos que esta simples abordagem é suficientemente para descrever seu comportamento qualitativo. A introdução de ruído à dinâmica do modelo também é analisada. Observa-se, neste caso, a existência de uma transição de fase entre um estado onde há um candidato majoritário (estado ordenado) e um estado onde todas as opiniões coexistem com aproximadamente o mesmo número de eleitores (estado desordenado), dependendo da intensidade desse ruído. Resultados de simulações de Monte Carlo numa rede de Barabási-Albert apresentam boa concordância quando confrontadas com resultados analíticos.

Palavras-chaves: opinião, consenso, Modelo Sznajd Complexo, equação mestra, rede de Barabási-Albert. 


\section{Abstract}

This work discusses, mainly with an analytical approach, the Sznajd Model and some of its variants. We propose a master equation that describes the evolution of opinions in the model, studying its possible steady states in a mean-field approximation. We show that this approach, although very simple, is enough to describe the qualitative behavior of the model. The introduction of noise in the dynamics is also studied in detail. In this case we show that there is a phase transition between an state in which a single candidate has the majority of the votes (ordered phase) and another one where the votes are well distributed among all the candidates (disordered phase), depending on the level of noise. Monte Carlo simulations in a Barabási-Albert network show good agreement with the analytical results.

Keywords: opinion, consensus, Complex Sznajd Model, master equation, Barabási-Albert network. 


\section{Sumário}

$\begin{array}{ll}\text { Agradecimentos } & 5\end{array}$

$\begin{array}{ll}\text { Resumo } & 7\end{array}$

$\begin{array}{ll}\text { Abstract } & 8\end{array}$

$\begin{array}{ll}\text { Lista de Figuras } & 23\end{array}$

$\begin{array}{lr}\text { Lista de Tabelas } & 26\end{array}$

$\begin{array}{lll}1 & \text { Introdução } & 27\end{array}$

2 Modelagem de Opiniões: Panorama Geral 31

2.1 Redes Complexas . . . . . . . . . . . . . . . . . . . 32

2.1.1 Definição e Propriedades dos Grafos . . . . . . . 32

2.2 Principais Modelos Envolvendo Grafos . . . . . . . . 35

2.2 .1 Grafos Aleatórios . . . . . . . . . . . . . 35

2.2 .2 Grafo Mundo Pequeno . . . . . . . . . . . . 37

2.2 .3 Rede de Barabási-Albert . . . . . . . . . . . . . 39

2.3 Propagação de Opiniões e a Estatística de Eleições Proporcionais . . . . . . . . . . . . . . 42

2.3.1 Comportamento Estatístico de Eleições Propor-

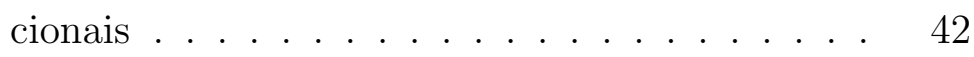


2.3.2 Modelo de Ising com Dinâmica de Glauber . . . 43

2.3.3 Modelo da Regra da Maioria . . . . . . . . . . . 47

2.3.4 Modelo do Votante Majoritário . . . . . . . . . 49

2.3.5 Modelo do Votante . . . . . . . . . . . . . . . . 50

2.4 O Modelo Sznajd e suas Variantes . . . . . . . . . . . 51

2.4.1 O Modelo Sznajd . . . . . . . . . . . . . . . . 51

2.4.2 Modificações do Modelo Sznajd . . . . . . . . . 53

2.4.3 O Modelo Sznajd Complexo . . . . . . . . . . . 55

2.4.4 Regras de Confiança Limitada - O Modelo Sznajd Generalizado. . . . . . . . . . . 58

2.4.5 Aproximação de Campo Médio em Sistemas Dinâmicos . . . . . . . . . . . . . . . 61

3 Aproximação de Campo Médio $\quad 67$

3.1 Aproximação de Campo Médio na Ausência de Ruído . 67

3.1.1 Construindo a Equação Mestra ... . . . . . . 67

3.1.2 Hipóteses para a Aproximação de Campo Médio 75

3.1.3 Determinando os Estados Estacionários . . . . . 77

3.2 Aproximação de Campo Médio com Ruído . . . . . . . 79

3.2.1 Construindo a Equação Mestra . . . . . . . . . 79

3.2.2 Determinando os Estados Estacionários . . . . . 81

3.3 Análise de Casos Limites . . . . . . . . . . . . . . . . . 87

3.3 .1 Campo Nulo. . . . . . . . . . . . . . . . . . . . 87

3.3 .2 Campo Máximo . . . . . . . . . . . . . . . . 88

3.3 .3 Campo Fraco . . . . . . . . . . . . . . . . . . . 88

3.3.4 Campo Forte . . . . . . . . . . . . . . . . . . . . . 89

3.4 Interpretação Física de $\tilde{w}$ e $w_{t} \ldots \ldots \ldots$. . . . . . . 90

3.5 Reconhecendo a Possibilidade de uma Transição de Fases de Segunda Ordem . . . . . . . . . . . . . . . . . . . . 92

3.6 Resultados para um Grafo Completo . . . . . . . . . 93 
3.7 Resultados para Redes Regulares _ . . . . . . . . . . . 97

3.7.1 Cadeia de Eleitores . . . . . . . . . . . . . . . 98

3.7 .2 Rede Quadrada . . . . . . . . . . . . . . . . . . 102

3.7.3 Rede Cúbica . . . . . . . . . . . . . . . . . . 106

3.7.4 Limite de Altas Dimensionalidades . . . . . . . 110

4 Simulações de Monte Carlo 113

4.1 Procedimento Numérico . . . . . . . . . . . . . . . . 114

4.1.1 Representações Numéricas da Rede . . . . . . . 114

4.1.2 Implementando a Dinâmica . . . . . . . . . . . 116

4.1.3 Cálculo dos Parâmetros Médios da Rede . . . . 118

4.1.4 Análise Numérica de Outras Redes . . . . . . . 127

4.2 Resultados Numéricos para o Modelo sem Ruído . . . . 136

4.2.1 Séries Temporais na Rede de Barabási-Albert . 136

4.2.2 Séries Temporais para Redes Regulares . . . . . 140

4.2.3 Séries Temporais em Grafos Completos . . . . . 146

4.2.4 Comportamento dos Indecisos _. . . . . . . . 148

4.3 Resultados Numéricos para o Modelo com Ruído . . . . 150

4.3.1 Séries Temporais na Rede de Barabási-Albert . 150

4.3.2 Diagrama de Bifurcação e Transição de Fases na Rede de Barabási-Albert . . . . . . . . . . . 157

5 Conclusões e Perspectivas 165

A Construindo a Rede de Barabási-Albert 169

B Ponteiro de Opiniões 173

$\begin{array}{ll}\text { C Representação de Vizinhos } & 174\end{array}$

$\begin{array}{ll}\text { D Representação de Número de Votos } & 177\end{array}$ 
E Acompanhando a Dinâmica do Modelo Sznajd Complexo

F Algoritmo Utilizado no Cálculo dos Parâmetros Médios da Rede

G Algoritmo Utilizado para a Realização da Dinâmica do Modelo Sznajd Complexo 


\section{Lista de Figuras}

2.1 Representação gráfica de um grafo com 5 vértices e 6 arestas. . . . . . . . . . . . . . . . 33

2.2 Padrões típicos para redes Mundo Pequeno considerando $k=2$ e diferentes valores de $\phi . \ldots \ldots \ldots . . \ldots 38$

2.3 Coeficiente de aglomeração A e distância média típica $L$ normalizados em função da probabilidade de reorientação $\phi$. Num certo intervalo nas vizinhanças de $\phi=$ 0,01 reproduz-se o regime mundo pequeno. . . . . . .

3.1 O sítio para o qual estamos escrevendo o termo da equação mestra está marcado em azul (e representado pelo índice $i$ ), sua primeira vizinhaça em verde (representados pelo índice $j$ ) e sua segunda vizinhaça em vermelho (representados pelo índice $j^{\prime}$ ). Eventualmente, alguns sítios que pertencem a segunda vizinhaça de $i$ não estão representados na figura. . . . . . . . . . . 68

3.2 Confronto direto entre $i$ e um de seus vizinhos $j$. (a) Primeiramente, um sítio $j \in \Gamma_{i}$ é escolhido. (b) Em seguida, o sítio sorteado assedia seus vizinhos. (c) O sítio $j$ escolhe o sítio $i$ fixado. (d) Finalmente, $j$ consegue convencer o sítio fixado a concordar consigo. . . . . . . 
3.3 Convencimento através da interação de vizinhos de primeira espécie. (a) Um sítio $j \in \Gamma_{i}$ é sorteado e, por sua vez, escolhe um de seus vizinhos $j^{\prime} \in \gamma_{i}$ de mesma opinião. (b) Ambos os sítios assediam suas respectivas vizinhanças. (c) O sítio $j$ escolhe o sítio $i$ fixado. (d) j consegue convencer o sítio fixado a concordar com a opinião do par. . . . . . . . . . . . . . . . 70

3.4 Convencimento através da interação de vizinhos de segunda espécie. (a) Primeiramente, o sítio $j^{\prime} \in \gamma_{i}$ é sorteado. (b) O sítio $j^{\prime}$, após assediar sua vizinhança, escolhe um de seus vizinhos $j \in \Gamma_{i}$. (c) $j$ escolhe o sítio $i$ fixado. (d) $j$ consegue convencer o sítio fixado a concordar com a opinião do par. . . . . . . . . . . . .

3.5 Principal diferença entre as concordâncias de primeira e segunda espécie. (a) Primeiramente, um sítio $j^{\prime} \in \gamma_{i}$ é escolhido. (b) O sítio $j^{\prime}$ assedia seus vizinhos. (c) $j^{\prime}$ escolhe um de seus vizinhos que pertence a terceira vizinhança do sítio $i$ fixado. (d) Neste caso, o par concordante não é capaz de interagir com o sítio $i$ que está 'fora de seu alcance'. . . . . . . . . . . . . . .

3.6 Diagrama de bifurcação típico para o Modelo Sznajd Complexo numa aproximação de campo médio. As linhas tracejadas representam soluções instáveis. . . . . .

3.7 Diagrama típico do parâmetro de ordem contra o parâmetro de controle para o Modelo Sznajd Complexo numa aproximação de campo médio. . . . . . . . . . . . . .

3.8 Diagrama de bifurcação para o Modelo Sznajd Complexo referente a um grafo completo com 10 candidatos numa aproximação de campo médio. As linhas tracejadas representam soluções instáveis. . . . . . . . . . . 
3.9 Diagrama de bifurcação para o Modelo Sznajd Complexo referente a um grafo completo com 2 candidatos numa aproximação de campo médio. As linhas tracejadas representam soluções instáveis. . . . . . . . . .

3.10 Diagrama de bifurcação para o Modelo Sznajd Complexo referente a um grafo completo com alta campetitividade entre os candidatos numa aproximação de campo médio. . . . . . . . . . . . . . . . . . .

3.11 Parâmetro de ordem contra o parâmetro de controle para o Modelo Sznajd Complexo referente a um grafo completo numa aproximação de campo médio com diferentes valores para o número de candidatos. . . . . . . . . .

3.12 Diagrama de bifurcação para o Modelo Sznajd Complexo referente a uma cadeia de eleitores com 10 candidatos numa aproximação de campo médio. As linhas tracejadas representam soluções instáveis. . . . . . . . .

3.13 Diagrama de bifurcação para o Modelo Sznajd Complexo referente a uma cadeia de eleitores com 2 candidatos numa aproximação de campo médio. As linhas tracejadas representam soluções instáveis. . . . . . . . . 100

3.14 Diagrama de bifurcação para o Modelo Sznajd Complexo referente a uma cadeia de eleitores com alta competitividade entre os candidatos numa aproximação de campo médio. . . . . . . . . . . . . . . .

3.15 Parâmetro de ordem contra o parâmetro de controle para o Modelo Sznajd Complexo referente a uma cadeia de eleitores numa aproximação de campo médio com diferentes valores para o número de candidatos. . . . . . . 102 
3.16 Diagrama de bifurcação para o Modelo Sznajd Complexo referente à rede quadrada com 10 candidatos numa aproximação de campo médio. As linhas tracejadas representam soluções instáveis. . . . . . . . . . . . . . . 103

3.17 Diagrama de bifurcação para o Modelo Sznajd Complexo referente à rede quadrada com 2 candidatos numa aproximação de campo médio. As linhas tracejadas representam soluções instáveis. . . . . . . . . . . . . . . . 104

3.18 Diagrama de bifurcação para o Modelo Sznajd Complexo referente à rede quadrada com alta competitividade entre os candidatos numa aproximação de campo médio. . . . . . . . . . . . . . . . . 105

3.19 Parâmetro de ordem contra o parâmetro de controle para o Modelo Sznajd Complexo referente à rede quadrada numa aproximação de campo médio com diferentes valores para o número de candidatos. . . . . . . . . . . 106

3.20 Diagrama de bifurcação para o Modelo Sznajd Complexo referente à rede cúbica com 10 candidatos numa aproximação de campo médio. As linhas tracejadas representam soluções instáveis. . . . . . . . . . . . 107

3.21 Diagrama de bifurcação para o Modelo Sznajd Complexo referente à rede cúbica com 2 candidatos numa aproximação de campo médio. As linhas tracejadas representam soluções instáveis. . . . . . . . . . . . . 108

3.22 Diagrama de bifurcação para o Modelo Sznajd Complexo referente à rede cúbica com alta competitividade entre os candidatos numa aproximação de campo médio. 109 
3.23 Parâmetro de ordem contra o parâmetro de controle para o Modelo Sznajd Complexo referente à rede cúbica numa aproximação de campo médio com diferentes valores para o número de candidatos. . . . . . . . . . . . 110

4.1 (a) Exemplo de uma Rede de Barabási-Albert com $N=$ 8 vértices e conectividade mínima $m=2$ arestas por vértice. (b) Representação de Arestas da rede desenhada em (a). . . . . . . . . . . . . . . .

4.2 (a) Exemplo de rede quadrada com $N=25$ eleitores. (b) Representação de Arestas referente aos quatro primeiros elementos da rede em (a). . . . . . . . . . . . . 129

4.3 Evolução temporal da distribuição de votos para o Modelo Sznajd Complexo sem Ruído com $N_{c}=10$ candidatos numa rede de Barabási-Albert com $N=1000$ eleitores e conectividade mínima $m=5$. . . . . . . 137

4.4 Evolução temporal da distribuição de votos para o Modelo Sznajd Complexo sem Ruído com $N_{c}=2$ candidatos numa rede de Barabási-Albert com $N=1000$ eleitores e conectividade mínima $m=5 . \quad$. . . . . . 139

4.5 Evolução temporal da distribuição de votos para o Modelo Sznajd Complexo sem Ruído com $N_{c}=10$ candidatos num anel de $N=1000$ eleitores. . . . . . . . . 141

4.6 Evolução temporal da distribuição de votos para o Modelo Sznajd Complexo sem Ruído com $N_{c}=2$ candidatos num anel de $N=1000$ eleitores. . . . . . . . . 142

4.7 Evolução temporal da distribuição de votos para o Modelo Sznajd Complexo sem Ruído numa rede quadrada com $N=1024$ eleitores e $N_{c}=10$ candidatos. . . . . . 143 
4.8 Evolução temporal da distribuição de votos para o Modelo Sznajd Complexo sem Ruído numa rede quadrada com $N=1024$ eleitores e $N_{c}=2$ candidatos. . . . . . . 144

4.9 Evolução temporal da distribuição de votos para o Modelo Sznajd Complexo sem Ruído com $N_{c}=10$ candidatos numa rede cúbica com $N=1000$ eleitores. . . 145

4.10 Evolução temporal da distribuição de votos para o Modelo Sznajd Complexo sem Ruído com $N_{c}=2$ candidatos numa rede cúbica com $N=1000$ eleitores. . . 146

4.11 Evolução temporal da distribuição de votos para o Modelo Sznajd Complexo sem Ruído com $N_{c}=10$ candidatos num grafo completo com $N=1000$ eleitores. . 147

4.12 Evolução temporal da distribuição de votos para o Modelo Sznajd Complexo sem Ruído com $N_{c}=2$ candidatos num grafo completo com $N=1000$ eleitores. . 148

4.13 Comportamento dos indecisos nas diferentes redes estudadas. As redes utilizadas obedeciam um padrão com $N=1000$ eleitores e $N_{c}=2$ candidatos. . . . . . . . 149

4.14 Evolução temporal da distribuição de votos para o Modelo Sznajd Complexo com Ruído numa Rede de Barabási-Albert com $N=1000$ eleitores e $N_{c}=10$ candidatos. (a) $w=0,01$ e (b) $w=0,10 \ldots \ldots 1$

4.15 Evolução temporal da distribuição de votos para o Modelo Sznajd Complexo com Ruído numa Rede de Barabási-Albert com $N=1000$ eleitores e $N_{c}=10$ candidatos. (a) $w=0,80$ e (b) $w=0,90 \ldots \ldots 2$ 
4.16 Evolução temporal da distribuição de votos para o Modelo Sznajd Complexo com Ruído numa Rede de Barabási-Albert com $N=10000$ eleitores e $N_{c}=10$ candidatos. (a) Fase ordenada $(w=0.1)$ e (b) Fase Desordenada $(w=0.9) \ldots \ldots \ldots \ldots$

4.17 Evolução temporal da distribuição de votos para o Modelo Sznajd Complexo com Ruído numa Rede de Barabási-Albert com $N=10000$ eleitores e $N_{c}=10$ candidatos. As linhas tracejadas representam os valores de campo médio para o estado estacionário do modelo. (a)

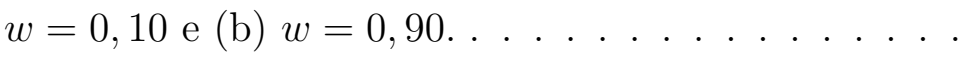

4.18 Evolução temporal da distribuição de votos para o Modelo Sznajd Complexo com Ruído numa Rede de Barabási-Albert com $N=10000$ eleitores e $N_{c}=10$ candidatos. As linhas tracejadas representam os valores de campo médio para o estado estacionário do modelo. (a) $w=0,025$ e (b) $w=0,975 \ldots \ldots$. . . . . . . 156

4.19 Diagrama de bifurcação numérico para o Modelo Sznajd Complexo com Ruído numa Rede de Barabási-Albert com $N=10000$ eleitores e $N_{c}=10$ candidatos. . . . . 158

4.20 Comparação entre os diagramas de bifurcação numérico e analítico para o Modelo Sznajd Complexo com Ruído numa Rede de Barabási-Albert com $N=10000$ eleitores e $N_{c}=10$ candidatos. . . . . . . . . . . . 158

4.21 Diagrama de bifurcação para o Modelo Sznajd Complexo numa Rede de Barabási-Albert com $N=10000$ eleitores e $N_{c}=10$ candidatos. Para a construção do diagrama consideramos o candidato majoritário e a soma dos votos dos demais candidatos. . . . . . . . . . . 160 
4.22 Comparação entre os diagramas de birfucação numérico e analítico para o Modelo Sznajd Complexo numa Rede de Barabási-Albert com $N=10000$ eleitores e $N_{c}=10$ candidatos. Para a construção dos diagramas consideramos o candidato majoritário e a soma dos votos dos demais candidatos. . . . . . . . . . . . . 161

4.23 Parâmetro de ordem numérico em função do parâmetro de controle para o Modelo Sznajd Complexo com Ruído numa Rede de Barabási-Albert com $N=10000$ eleitores e $N_{c}=10$ candidatos. O modelo apresenta uma transição de fases de primeira ordem entre as fases de consenso e a fase ruidosa com ponto crítico igual a $w_{t}=0,287 \pm$ $0,001 \ldots \ldots \ldots \ldots \ldots \ldots$

4.24 Comparação entre o parâmetro de ordem numérico e o parâmetro de ordem analítico em função do parâmetro de controle para o Modelo Sznajd Complexo com Ruído numa Rede de Barabási-Albert com $N=10000$ eleitores e $N_{c}=10$ candidatos. . . . . . . . . . . . . . . . 164

A.1 Parte do código fonte responsável pela construção da configuração inicial da rede de Barabási-Albert. . . . . 170

A.2 Parte do código fonte responsável pela continuidade na construção da rede de Barabási-Albert. . . . . . . . . . 171

A.3 Saída padrão do ponteiro ${ }^{* *} \mathrm{M}$ ilustrando uma rede de Barabási-Albert $N=11$ vértices e conectividade mínima $m=2$. A linha 4 destaca a configuração inicial do restante da rede. . . . . . . . . . . . . . . . . 172 
B.1 Saída padrão para o ponteiro de opiniões de uma rede de Barabási-Albert com $N=15$ e $N_{c}=5$. A linha 1 representa a configuração da rede correspondente ao processo de semeadura e a linha hachurada indica que a dinâmica atingiu o estado estacionário de consenso com todos os eleitores escolhendo o candidato 3. Cada linha está associada a um instante medido em Tempo de Monte Carlo. . . . . . . . . . . . . . . . . . 173

C.1 Trecho do código fonte que contabiliza o grau de cada vértice. Q $[\mathrm{i}]$ é o grau do vértice i. . . . . . . . . . . . . 174

C.2 Trecho do código fonte responsável pela construção da Representação de Vizinhos. . . . . . . . . . . . . . . . 175

C.3 Saída padrão do ponteiro ${ }^{*} \mathrm{Q}$ para a rede de BarabásiAlbert com $m=2$ e $N=11$ apresentada anteriormente na Figura A.3. O elemento da primeira coluna de cada linha representa um dado sítio da rede e o elemento da segunda coluna o seu respectivo grau, por exemplo, o sítio 0 tem grau $Q[0]=5$. . . . . . . . . . . . 175

C.4 Saída padrão do ponteiro **A para a rede de BarabásiAlbert com $m=2$ e $N=11$ apresentada anteriormente na Figura A.3. . . . . . . . . . . . . . . 176

D.1 Saída padrão do ponteiro * Nvotos para uma rede de Barabási-Albert com $N=15$ e $N_{c}=5$. A coluna 1 indica o instante particular da dinâmica, a coluna 2 indica o número de indecisos e as demais colunas representam cada candidato em ordem de enumeração. . . . . . . . 177 
E.1 Saída padrão referente ao ponteiro $*$ Op ilustrando a dinâmica do Modelo Sznajd em seu primeiro Tempo de Monte Carlo. O fato da rede possuir $N=5$ eleitores determina as 5 interações entre os sítios correspondentes a cada Passo de Monte Carlo. . . . . . . . . . . . . .

E.2 Saída padrão referente ao ponteiro *Op ilustrando o segundo instante da dinâmica iniciada na Figura E.1. . . 180

E.3 Saída padrão referente ao ponteiro *Op ilustrando o terceiro instante da dinâmica iniciada na Figura E.1. . . . 181

E.4 Saída padrão referente ao ponteiro *Op ilustrando o quarto instante da dinâmica iniciada na Figura E.1. . . 182

E.5 Saída padrão referente ao ponteiro $*$ Op ilustrando o quinto instante da dinâmica iniciada na Figura E.1. É notável o aumento na frequência de interações entre vizinhos concordantes.

E.6 Saída padrão referente ao ponteiro *Op ilustrando o sexto instante da dinâmica iniciada na Figura E.1. A partir deste instante, a dinâmica atinge seu estado estacionário de consenso com todos os eleitores votando no candidato 1. . . . . . . . . . . . . .

E.7 Representação de Opiniões do instante $t=1$ TMC para o Modelo Sznajd Complexo com Ruído numa rede de Barabási-Albert com $N=5$ eleitores, $N_{c}=2$ candidatos e $w=0,1 \ldots \ldots \ldots \ldots$

E.8 Representação de Opiniões do instante $t=6$ TMC para o Modelo Sznajd Complexo com Ruído numa rede de Barabási-Albert com $N=5$ eleitores, $N_{c}=2$ candidatos e $w=0,1$. Neste instante, o sistema assume um temporário estado de consenso. . . . . . . . . . . . 186 
E.9 Representação de Opiniões do instante $t=8$ TMC para o Modelo Sznajd Complexo com Ruído numa rede de Barabási-Albert com $N=5$ eleitores, $N_{c}=2$ candidatos e $w=0,1$. A linha hachurada destaca a destruição do estado de consenso assumido pelo sistema no instante $t=6$ TMC . . . . . . . . . . . . 187

E.10 Representação de Opiniões para o Modelo Sznajd Complexo com Ruído Máximo em seus instantes iniciais. Perceba que a dinâmica convencional é completamente abandonada. . . . . . . . . . . . . . . . . . 188

E.11 A linha hachurada destaca a Representação de Opiniões para o Modelo de Sznajd Complexo numa rede de Barabási-Albert com $N=5$ eleitores, $N_{c}=2$ candidatos e $w=1$ no instante $t=10$ TMC. O intervalo de tempo decorrido desde o instante inicial até o instante considerado é longo o suficientemente para considerarmos o sistema em regime estacionário. Perceba que, aproximadamente, os dois candidatos apresentam a mesma quantidade de votos. . . . . . . . . . . . . . . . . . . . . 189 


\section{Lista de Tabelas}

2.1 Tabela resumindo as etapas da dinâmica de convencimentos do Modelo Sznajd Complexo. . . . . . . . . . 57

2.2 Tabela resumindo as etapas da dinâmica de convencimentos do Modelo Sznajd Complexo com a inclusão do ruído. . . . . . . . . . . . . . . . . . . . 58

3.1 Resumo dos principais resultados obtidos para diferentes redes e numa aproximação de campo médio para o Modelo Sznajd Complexo. . . . . . . . . . . . . . . . . . 112

3.2 Valores assumidos pelos parâmetros médios que caracterizam as diferentes redes abordadas numa aproximação de campo médio para o Modelo Sznajd Complexo. . . . 112

4.1 Algoritmo empregado na implementação da dinâmica de convencimentos do Modelo Sznajd Complexo. . . . . . 117

4.2 Principais etapas na construção numérica da dinâmica de convencimentos do Modelo Sznajd Complexo com Ruído. . . . . . . . . . . . . . . . . . . . . . . . . 118

4.3 Representação de vizinhos e distribuição de graus para a rede-exemplo da Figura 4.1. . . . . . . . . . . . . . 121 
4.4 Representação de segundos vizinhos e distribuição para o número de segundos vizinhos para a rede-exemplo da Figura 4.1. . . . . . . . . . . . . . . . . .

4.5 Distribuição de graus dos primeiros vizinhos para a redeexemplo da Figura 4.1. . . . . . . . . . . . . . . . 123

4.6 Frações de arestas compartilhadas entre os vértices da segunda e da primeira vizinhança para cada vértice de nossa rede-exemplo. . . . . . . . . . . . . . . . . . . . 125

4.7 Distribuição de fatores de acoplamento parcial para a rede-exemplo da Figura 4.1. . . . . . . . . . . . . 125

4.8 Amostra de resultados numéricos para os parâmetros médios de uma rede de Barabási-Albert com N=10.000 eleitores e conectividade mínima $\mathrm{m}=5$ entre eles. Consideramos um ensemble constituído de 100 simulações da rede.

4.9 Representação de vizinhos e distribuição de graus para a rede quadrada representada na Figura 4.2. . . . . . . 130

4.10 Número de segundos vizinhos para os vértices da rede quadrada apresentada na Figura 4.2. . . . . . . . . . 131

4.11 Resultados numéricos dos parâmetros médios para a rede quadrada da Figura 4.2. . . . . . . . . . . . . 132

4.12 Representação de vizinhos e distribuição de graus para um grafo completo com $\mathrm{N}=10$ candidatos. . . . . . . 132

4.13 Representação de segundos vizinhos para um grafo completo com $\mathrm{N}=10$ eleitores. O algoritmo retornava -1 na inexistência de segundos vizinhos. . . . . . . . . . . 133

4.14 Parâmetros críticos para o Modelo Sznajd Complexo numa aproximação de campo médio considerando as vizinhanças $\gamma$ e $\tilde{\gamma}$ de um grafo completo. . . . . . . . . . 135 
4.15 Tempos de relaxação em diferentes redes para o Modelo Sznajd Complexo sem Ruído. O índice sobrescrito refere-se ao número de candidatos considerados na rede e o índice 0 subescrito refere-se ao tempo de relaxação dos indecisos. . . . . . . . . . . . . . . . 150

4.16 Resultados numéricos para os parâmetros médios da rede de Barabási-Albert. Os valores médios foram estabelecidos a partir de um ensemble com 100 realizações numéricas de redes com $N=10000$ vértices e conectividade mínima $m=5 . \quad \ldots \ldots \ldots \ldots \ldots$

4.17 Intensidades críticas de ruído para as diferentes redes estudadas. Para o estabelecimento dos resultados na rede BA nos utilizamos de um ensemble com 100 realizações de redes com $N=10000$ vértices e $N_{c}=10$ candidatos. 162 


\section{Capítulo 1}

\section{Introdução}

O Modelo Sznajd, proposto em 2000 por Katarzina Sznajd-Weron e Jósef Sznajd, é um modelo que tem sido empregado com bastante sucesso na reprodução de algumas propriedades estatísticas aparentemente universais observadas em eleições proporcionais e majoritárias. Ele é, essencialmente, um modelo de Ising, com uma dinâmica específica, que sempre evolui para um estado estacionário de consenso. Sua originalidade reside na maneira que os sítios interagem: dois sítios concordantes influenciam a opinião dos seus vizinhos de maneira a fazêlos concordar consigo ao invés de atualizarem suas opiniões a partir da de seus vizinhos, como no modelo do votante $[1,2]$.

Este modelo pretende incorporar efeitos potencializadores na capacidade de convencimento originados na existência de grupos de indivíduos de mesma opinião (validação social) e tem se mostrado como adequado para explicar o comportamento de escala (lei de potência) observado na distribuição de candidatos com determinado número de votos [1]. Tal comportamento foi descoberto em 1999 por Costa-Filho e colaboradores, e corroborado por estudos posteriores de eleições proporcionais realizadas não só no Brasil como em outros países, sendo portanto robusto [3]. 
Nas últimas décadas, este modelo tem sido bastante estudado, seja com suas regras originais ou em diferentes versões, nas quais elas são alteradas a fim de incluir determinados aspectos relevantes, tais como a possibilidade de mais de duas opiniões, existência de inomogeneidades na rede ("cabos eleitorais"), limitação da capacidade de convencimento dos eleitores, diferenças de topologia na rede que define o relacionamento entre os eleitores, dentre outras [2, 4]. Em 2002, Bernardes e colaboradores modificaram o modelo Sznajd, propondo um novo modelo que vamos denominar Modelo Sznajd Complexo, estudando-o numa rede livre de escala do tipo Barabási-Albert e verificaram que, embora o seu estado estacionário apresente comportamento trivial, seu transiente é capaz de descrever a lei de potência observada por Costa-Filho $[5]$.

É importante destacar que a maior parte das publicações exploram o modelo Sznajd através de abordagens numéricas, sendo que existem poucas abordagens analíticas. É nesse contexto que se insere essa dissertação, com parte do trabalho desenvolvido pelo grupo de pesquisa de minha orientadora em um esforço para desenvolver uma abordagem analítica para o modelo.

Em trabalhos anteriores que resultaram na tese de mestrado de Fábio Vannucchi em 2006 [6], Vannucchi e Prado analisaram as propriedades do transiente do modelo Sznajd em redes regulares, redes livre de escala do tipo Barabási-Albert e redes mundo pequeno, com o objetivo de entender as influências do processo de semeadura (escolha dos eleitores iniciais) e da topologia da rede no resultado final do processo (o candidato vencedor). Também propuseram uma equação mestra bastante simplificada para a evolução temporal do modelo, que foi resolvida tanto analiticamente em uma aproximação de campo médio como por simulações numéricas. Embora os resultados obtidos na aproximação de campo médio tenham explicado qualitativamente bem 
o comportamento do modelo, diversas discrepâncias foram observadas entre as soluções analítica e numérica.

Em 2009, Timpanaro e Prado [4] desenvolveram uma generalização para o Modelo Sznajd Complexo verificando ser a abordagem adotada em [6] exageradamente reducionista, sugerindo a possibilidade desta ser a origem do comportamento anômalo observado anteriormente.

Ao longo desta dissertação, pretendemos revisitar a equação mestra prospota por Vannucchi e Prado em [6], incluindo termos ignorados em sua abordagem e mostrando que a ausência deles é, de fato, a responsável pelas discrepâncias encontradas. Através de uma aproximação de campo médio, estudaremos os estados estacionários da nova equação proposta, para as versões com e sem ruído, e demonstraremos que este tratamento é mais adequado para descrever as propriedades do modelo.

Técnicas de Sistemas Dinâmicos serão utilizadas para estabelecer o diagrama de bifurcação no estado estacionário do modelo com ruído. Verifica-se uma descontinuidade neste diagrama, o que sugere uma transição de primeira ordem entre uma fase com um vencedor hegemônico (fase ordenada) e uma fase de empate técnico entre os candidatos (fase desordenada) em função da intensidade do campo aplicado. Além disso, resultados numéricos utilizando algoritmos de Monte Carlo numa rede de Barabási-Albert serão apresentados e confrontados com resultados analíticos.

No capítulo 2, apresentaremos um panorama geral de alguns dos principais modelos utilizados na modelagem de propagação de opiniões, de alguns conceitos básicos da teoria dos grafos e dos processos dinâmicos em redes complexas. Desenvolveremos ainda uma visão mais específica do problema em questão, apresentando o modelo Sznajd, seus variantes mais relevantes para esse estudo e estabelecidos recentemente na literatura.

No capítulo 3, desenvolveremos a aproximação de campo médio para 
a equação mestra que descreve o Modelo Sznajd Complexo. Estudaremos, em detalhes, as propriedades dos estados estácionários para a dinâmica do modelo nesta aproximação, enfatizando suas interpretações físicas.

No capítulo 4, apresentaremos resultados numéricos para o Modelo Sznajd Complexo numa rede de Barabási-Albert e o confronto destes resultados com os resultados analíticos do capítulo anterior.

Finalmente, no capítulo 5, apresentaremos as conclusões e perspectivas de continuidade. 


\section{Capítulo 2}

\section{Modelagem de Opiniões: Panorama Geral}

Neste capítulo, iremos fazer uma rápida revisão dos conceitos fundamentais empregados nessa dissertação. Primeiramente, apresentaremos alguns conceitos da teoria dos grafos, em particular das redes complexas, pois é natural interprertarmos uma comunidade ou colégio eleitoral como uma rede, em que os vértices (sítios) representariam os eleitores e as arestas representariam as relações sociais estabelecidas entre eles.

Na sequência, apresentaremos alguns modelos de evolução de opiniões muito estudados na literatura de física das últimas décadas, como o modelo do votante, o modelo Sznajd e os modelos de confiança limitada (bounded confidence). Daremos especial destaque ao modelo de Sznajd, mencionando suas origens, variações e principais diferenças com relação aos outros modelos, uma vez que é o modelo que estudaremos nessa dissertação. Por fim, mostraremos que a evolução das opiniões pode ser descrita por equações mestras na forma de equações de diferenças finitas lineares e de primeira ordem, pelas quais podemos determinar os estados estácionários segundo técnicas de sistemas 
dinâmicos.

\subsection{Redes Complexas}

Nesta seção pretendemos revisar o rápido e recente progresso no entendimento de redes utilizando idéias e métodos da Mecânica Estatística [7]. Descreveremos algumas das propriedades mais comuns na caracterização de redes relacionadas à Física, Matemática e Ciências Sociais [8].

\subsubsection{Definição e Propriedades dos Grafos}

Um grafo (ou rede) $\mathrm{G}$ pode ser entendido como um conjunto de vértices $\mathrm{V}$ e por um conjunto de arestas $\mathrm{A}$ representando as conexões estabelecidas entre esses vértices. Um grafo pode representar, por exemplo, uma rede de contatos sexuais [9], onde as pessoas são representadas pelos vértices e as arestas representam as relações sexuais praticadas entre elas. Grafos podem ser não-direcionados (como o grafo de relações sexuais citado anteriormente), onde as arestas são simétricas, ou podem ser direcionados, onde ordem dos vértices incididos passa a ser relevante [8]. Um exemplo de um grafo direcionado é uma teia alimentar entre diferentes espécies de um ecossistema, onde o fato de uma espécie X ser predadora de uma espécie Y não implica em Y ser predadora de $\mathrm{X}[8,10]$.

Formalmente, podemos definir um grafo $G$ como um conjunto de vértices $\mathrm{V}$, diferente do vazio, um conjunto de arestas $\mathrm{A}$ e uma aplicação $g: A \rightarrow P(V)$, que associa a cada aresta um subconjunto de dois elementos de $\mathrm{V}$, interpretado como as extremidades da aresta ${ }^{1}$. Por construção, existe um isomorfismo entre o conjunto de vértices $\mathrm{V}$ e

\footnotetext{
${ }^{1}$ Uma simples modificação na aplicação g permite generalizar esta definição para grafos direcionados.
} 
o conjunto imagem de g, $\operatorname{Im}(\mathrm{g})$, permitindo eventuais abusos de linguagem na identificação dos mesmos [11].

Na Figura 2.1, ilustramos a representação gráfica de um grafo em que $V=\{1,2,3,4,5\}, A=\{\{1,2\},\{1,5\},\{2,3\},\{2,4\},\{2,5\},\{3,5\}\}$ e g é escolhida como a identidade ${ }^{2}$.

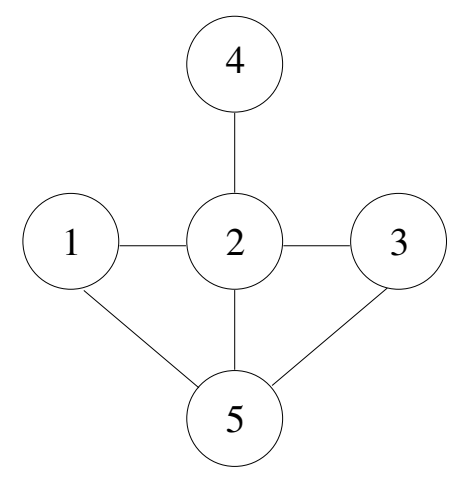

Figura 2.1: Representação gráfica de um grafo com 5 vértices e 6 arestas.

O grau de um vértice é o número de arestas que incidem sobre ele ${ }^{3}$. No caso da Figura 2.1, por exemplo, o grau do vértice 1 é igual a 2, enquanto que o grau do vértice 2 é igual a 4.

Dois vértices são considerados vizinhos se existe uma aresta entre eles. No grafo acima, os vértices 2 e 5 são vizinhos do vértice 1 , enquanto que os vértices 3 e 4 não o são. A vizinhança de um vértice $i$ é definida como o conjunto $\Gamma_{i}$ de vértices adjacentes a ele. Em nosso grafo-exemplo a vizinhança do vértice 1 é $\Gamma_{1}=\{2,5\}$.

Denominamos de caminho a sequência de vértices tal que, à excessão do último, exista uma aresta entre cada um deles e o vértice seguinte ${ }^{4}$. O comprimento do caminho é definido como o número de vértices que o

\footnotetext{
${ }^{2}$ Neste caso, o isomorfismo entre A e $\operatorname{Im}(\mathrm{g})$ é explorado na sua forma mais natural.

${ }^{3}$ Para grafos direcionados, distingue-se entre grau de entrada (número de aresta que entram no vértice) e grau de saída (número de vértices que saem do vértice).

${ }^{4} \mathrm{O}$ caminho é simples se nenhum dos vértices se repete ao longo da sequência.
} 
constitui. Desta forma, podemos redefinir vizinhança de um sítio $i$, de maneira equivalente à anterior, como o conjunto de vértices que distam uma unidade deste vértice [11].

Esta nova abordagem do conceito de vizinhança permite facilmente a generalização de vizinhança para ordens superiores. Assim, definimos vizinhança de ordem $\mathrm{k}$ de um vértice $i, \Gamma_{i}^{(k)}$, como o conjunto de vértices que distam $\mathrm{k}$ unidades deste vértice e que não pertençam a nenhuma vizinhança de ordem inferior. Em particular, a segunda vizinhança de um vértice $i, \Gamma_{i}^{(2)}$, é o conjunto de vértices que distam duas unidades de $i$ e não pertencem a sua primeira vizinhanca ${ }^{5}$.

Além disso, grafos apresentam uma extensa classificação quanto ao inter-relacionamento entre seus vértices e arestas. Vejamos algumas [11]:

- Grafos Simples e Multigrafos - Um grafo simples é um grafo não direcionado em que existe no máximo uma aresta entre quaisquer dois vértices. Em oposição, multigrafos são aqueles que apresentam arestas múltiplas (arestas paralelas). Para um grafo simples, o número de vizinhos de um vértice é igual ao seu grau (conectividade).

- Grafo Regular - é um grafo em que todos os vértices tem o mesmo grau.

- Grafo Completo - é o grafo simples em que, para cada vértice do grafo, existe uma aresta conectando este vértice a cada um dos demais, ou seja, todos os vértices do grafo possuem o mesmo grau, que é o máximo possível. O grafo completo de $\mathrm{N}$ vértices é frequentemente denotado por $K_{N}$. Ele tem $N(N-1) / 2$ arestas que correspondem a todas as possíveis escolhas de pares de vértices.

- Grafo Conexo - é o grafo em que é possível estabelecer um caminho entre qualquer par de vértices dado. O grafo-exemplo da Figura 2.1 é

\footnotetext{
${ }^{5}$ Podemos definir vizinhança de ordem nula como o próprio vértice, ou seja, $\Gamma_{i}^{(0)}=\{i\}$.
} 
do tipo conexo.

- Grafo Acíclico - é o grafo que não apresenta cíclos. Por definição, ciclo é um caminho que se inicia e termina no mesmo vértice. Em nosso grafo-exemplo, a sequência $(1,2,5,1)$ é um ciclo de comprimento 3 , portanto o grafo não é acíclico.

- Árvore - é um grafo acíclico e conexo. O exemplo clássico é a árvore genealógica dos ascendentes de um indivíduo. Entretanto, o exemplo mais notável em aplicações físicas é a Árvore de Cayley [7].

Em geral, para redes que modelam situações realistas, o número de vértices e arestas tendem a ser muito grandes (da ordem de milhões ou até mesmo bilhões de vértices) e uma sistematização a partir de conceitos estatísticos passa a ser conveniente [8]. Destacamos, a seguir, algumas propriedades de grafos como o efeito de mundo pequeno (smallworld), redes livres de escala (scale-free) e índice de clusterização através dos modelos mais utilizados em aplicações físicas envolvendo grafos.

\subsection{Principais Modelos Envolvendo Grafos}

Nesta seção, apresentaremos uma breve descrição dos resultados mais importantes da teoria dos grafos na modelagem de sistemas físicos, destacando os aspectos diretamente relacionados a redes complexas.

\subsubsection{Grafos Aleatórios}

Erdös e Rény propuseram um dos modelos pioneiros para a modelagem de redes reais complicadas e irregulares denominado grafo aleatório [12]. Nele, é definido um emsemble de grafos $G_{N, p}$, no qual o número de vértices $\mathrm{N}$ é fixo e cada par de vértices pode ser conectado por uma aresta com probabilidade p. Desta forma, a distribuição de graus $P(k)$, 
ou seja, a probabilidade de um determinado vértice apresentar grau k, é dada pela distribuição binomial

$$
P(k)=\left(\begin{array}{c}
N-1 \\
k
\end{array}\right) p^{k}(1-p)^{N-k-1} \approx \frac{\lambda^{k} e^{-\lambda}}{k !},
$$

onde a última expressão é uma distribuição de Poisson e corresponde ao limite termodinâmico com grau médio $\lambda=(N-1) p$ fixo.

Um das principais características dos grafos aleatórios é a capacidade de reproduzir bem o comportamento de mundo pequeno apresentado por redes sociais, de acordo com trabalhos do sociólogo Milgram [13]. Uma rede é dita exibir o efeito de mundo pequeno quando a maioria dos pares de vértices estão unidos por um caminho relativamente curto na rede (muito menor do que o número de vértices da rede).

A distância típica entre os sítios de um grafo aleatório pode ser obtida através do seguinte argumento heurístico: o número $n$ de vértices que distam $d$ de um vértice específico é da ordem de $n \sim \lambda^{d}$, logo, o valor de $d$ necessário para percorrer a rede inteira satisfaz $\lambda^{d} \approx N$, de maneira que a distância típica para atravessar a rede é da ordem de $d \approx \ln N / \ln \lambda$. Portanto, a distância típica entre dois vértices cresce de modo proporcional a $\ln N \ll N$, satisfazendo o critério de mundo pequeno [6].

Apesar do distanciamento relativamente pequeno entre os vértices, eles não estão densamente conectados pela rede. A definição precisa desta propriedade é realizada através de um indicador denominado coeficiente de aglomeração, A, definido como a probabilidade média de dois vértices conectados a um terceiro compartilharem uma conexão. Para grafos aleatórios, como a probabilidade de dois vértices estarem conectados é constante e igual a p, independentemente deles compartilharem um vizinho em comum, o coeficiente de aglomeração é imediatamente determinado como sendo $A=p=\frac{\lambda}{N-1} \approx \frac{\lambda}{N}$, que tende a zero no limite termodinâmico. 
Embora os grafos aleatórios apresentem a propriedade mundo pequeno, o comportamento da distribuição de graus e do coeficiente de aglomeração divergem do comportamento das redes comumente encontradas na natureza, conhecidas genericamente como redes complexas, para as quais a distribuição de graus é invariante por escala e o coeficiente de aglomeração é alto $[6,10]$.

Desta forma, grafos aleatórios são raramente considerados em sua plenitude, sendo usado apenas para adquirir uma intuição inicial sobre o comportamento de redes e como ponto de partida conceitual para outros modelos mais sofisticados por ser mais acessível a uma abordagem analítica [10].

\subsubsection{Grafo Mundo Pequeno}

Em 1998, Watts e Strogatz propuseram um modelo de grafo que tinha como principal objetivo gerar uma rede com pequena distância típica entre os vértices mas com alto coeficiente de aglomeração [7]. O algoritmo utilizado na construção consiste, essencialmente, em duas etapas [7]:

1. Começar com ordem - Consideramos inicialmente um anel com $N$ vértices no qual cada um deles está conectado aos seus $k$ primeiros vizinhos ( $k / 2$ para cada lado). Por construção, consideramos $N \gg k \gg \ln (N) \gg 1$, a fim de que a distância típica entre os vértices seja muito menor do que o tamanho da rede.

2. Introduzir aleatoriedade - Com probabilidade $\phi$, reorientamos as arestas aleatoriamente entre os vértices de maneira que autoconexões e arestas duplas não sejam permitidas.

Na Figura 2.2 ilustramos redes mundo pequeno para alguns valores da probabilidade de reorientação $\phi[7]$. 


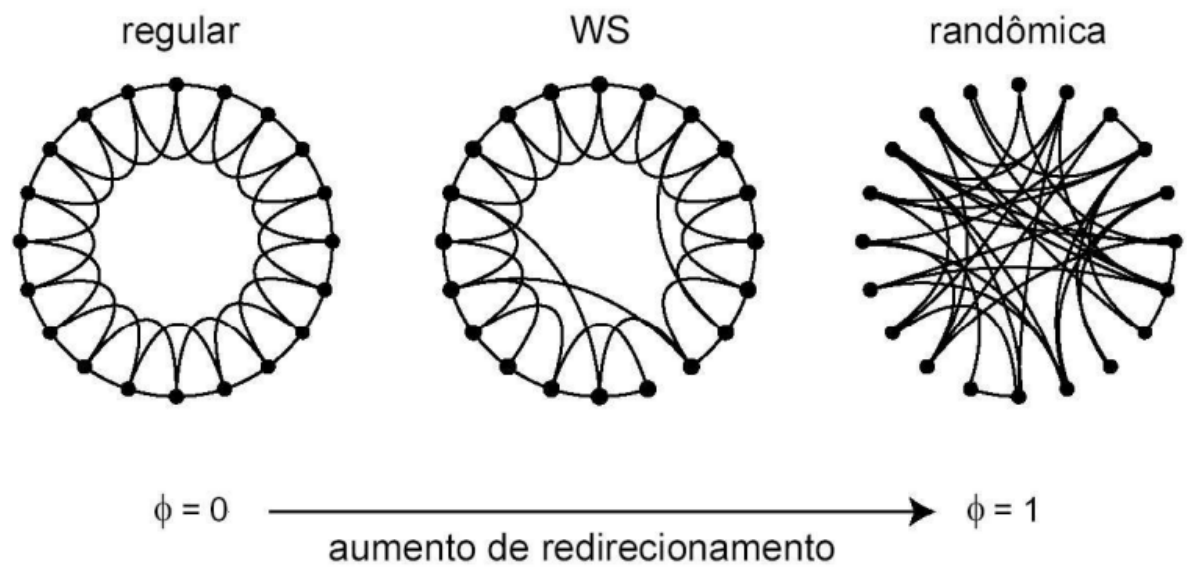

Figura 2.2: Padrões típicos para redes Mundo Pequeno considerando $k=2$ e diferentes valores de $\phi$.

Com esta construção, observa-se que, para um certo intervalo de $\phi$, o coeficiente de aglomeração é alto e a distância média entre os vértices é pequena, caracterizando o regime mundo pequeno, conforme a Figura $2.3[7]$. 


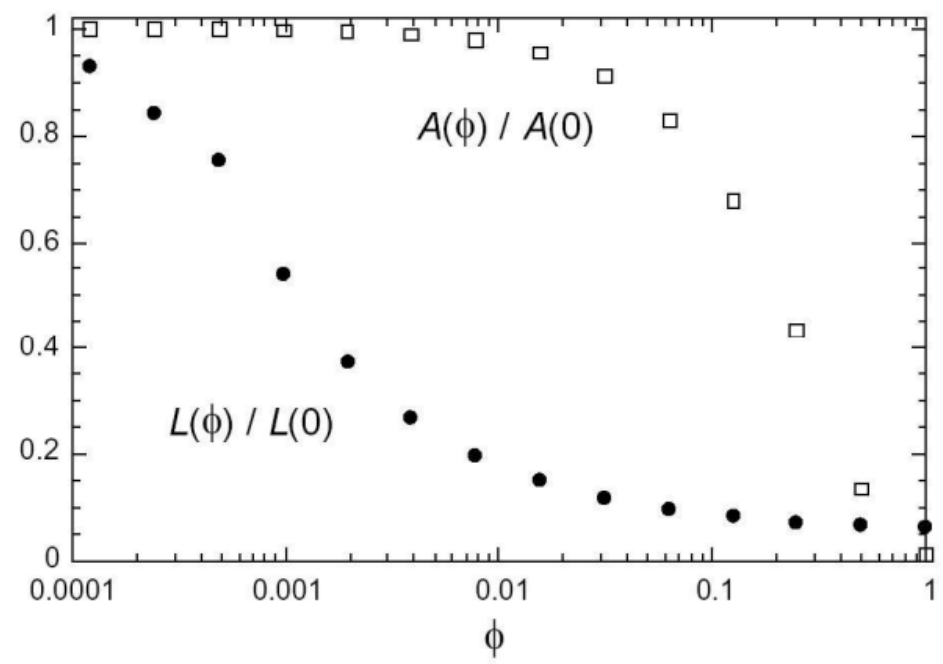

Figura 2.3: Coeficiente de aglomeração A e distância média típica $L$ normalizados em função da probabilidade de reorientação $\phi$. Num certo intervalo nas vizinhanças de $\phi=0,01$ reproduz-se o regime mundo pequeno.

Esta proposta obteve grande sucesso ao conciliar duas características muito desejáveis a um modelo físico realísta, representando um avanço em relação ao grafo aleatório. Entretanto, sua distribuição de graus é muito próxima a das redes regulares, impossibilitando a reprodução do caráter livre de escala na forma de lei de potências $[7,10]$.

\subsubsection{Rede de Barabási-Albert}

O mecanismo de conexão preferencial é predominantemente aceito como explicação mais provável para a geração de redes livres de escala $^{6}$, como redes de citação de artigos, Internet e a Rede Mundial de Computadores (World Wide Web) [7]. Apesar de ter sido proposto

\footnotetext{
${ }^{6} \mathrm{O}$ termo rede livre de escala é um tanto infeliz, pois, apesar da distribuição de graus de uma rede ser 'livre de escala', outras propriedades da rede podem estar relacionadas à escala.
} 
originalmente por Yule [14] e posteriormente por Price [15], o mecanismo só começou a chamar atenção de grande parte da comunidade científica através de seu redescobrimento por Barabási e Albert [16].

O grafo de Barabási e Albert ou rede BA é um grafo simples e não direcionado definido por dois princípios fundamentais [7]:

1. Mecanismo de Crescimento - O modelo se inicia a partir de um grafo completo $K_{m_{0}}$ com um pequeno número $m_{0}$ de vértices e, a cada iteração, um vértice com $m<m_{0}$ arestas é adicionado a rede estabelecendo conexões (arestas) com $m$ diferentes nós já presentes no sistema.

2. Conexão Preferencial - A probabilidade $\Pi\left(k_{i}\right)$ do novo vértice conectar-se a um vértice $i$ já presente no sistema é proporcional ao grau $k_{i}$ deste vértice, ou seja,

$$
\Pi\left(k_{i}\right)=\frac{k_{i}}{\sum_{j} k_{j}}
$$

Após t iterações, este procedimento resulta em uma rede com $N=$ $N_{0}+t$ vértices e $a=m_{0}+m t$ arestas. Simulações numéricas indicam que a distribuição de graus da rede resultante segue uma lei de potências na forma $\Pi(k)=k^{-\gamma}$, cujo expoente é aproximadamente igual a $\gamma_{B A}=3$, independentemente do valor de $\mathrm{m}$ [7]. Esta lei de potências pode ser obtida analiticamente, para valores assintóticos da conectividade $\mathrm{k}$ e no limite termodinâmico, como segue $[6,10]$.

A probabilidade de uma nova aresta se conectar a um vértice de grau $\mathrm{k}$ é dada por $^{7}$

$$
\frac{k \Pi_{k}}{\sum_{k} k \Pi_{k}}=\frac{k \Pi_{k}}{2 m}
$$

\footnotetext{
${ }^{7}$ Denotaremos a probabilidade de conexão a um sítio de grau $k_{i}$ simplesmente por $\Pi_{k_{i}}$.
} 
onde $\sum_{k} k \Pi_{k}=\langle k\rangle=2 m$ é o grau médio do grafo, pois cada vértice introduzido contribui com $2 m$ na contagem de graus.

Portanto, o número médio de vértices de grau $\mathrm{k}$ que ganham uma aresta quando adicionamos um novo vértice a rede é

$$
m \frac{k \Pi_{k}}{2 m}=\frac{k \Pi_{k}}{2}
$$

que independe de m.

Assim, após a adição de um vértice, a quantidade de vértices com grau k sofre uma redução, em média, para $N \Pi_{k}-\frac{1}{2} k \Pi_{k}$. Por outro lado, uma parte dos vértices de grau k-1 pode sofre um aumento médio de $\frac{1}{2} k \Pi_{k}$, exceto para vértices com grau $\mathrm{m}$, onde a taxa de entrada é exatamente 1 , correspondendo somente ao novo vértice adicionado. Portanto, o saldo de vértices por grau se torna

$$
(N+1) \Pi_{k, N+1}-N \Pi_{k, N}=\left\{\begin{array}{cc}
\frac{1}{2}(k-1) \Pi_{k-1, N}-\frac{1}{2} k \Pi_{k, N} & , k>m \\
1-\frac{1}{2} m \Pi_{k, N} & , k=m
\end{array}\right.
$$

em que $\Pi_{k, N}$ representa a distribuição de graus quando a rede tinha $\mathrm{N}$ vértices.

A lei de potências pode ser obtida ao investigarmos os estados estacionários deste sistema de equações de diferenças, ou seja, as sequências que satisfazem $\Pi_{k, N+1}=\Pi_{k, N}=\Pi_{k}$.

$$
\begin{gathered}
\Pi_{k}=\left\{\begin{array}{cc}
\frac{1}{2}(k-1) \Pi_{k-1}-\frac{1}{2} k \Pi_{k} & , k>m \\
1-\frac{1}{2} m \Pi_{k} & , k=m
\end{array}\right. \\
\Rightarrow\left\{\begin{array}{c}
\Pi_{m}=\frac{2}{m+2} \\
\Pi_{k}=\frac{k-1}{k+2} \Pi_{k-1}
\end{array}\right.
\end{gathered}
$$


Assim, concluímos que

$$
\Pi_{k}=\frac{(k-1)(k-2) \ldots m}{(k+2)(k+1) \ldots(m+3)} \Pi_{m}=\frac{2 m(m+1)}{(k+2)(k+1) k} \sim k^{-3},
$$

de acordo com discussão anterior.

Apesar da capacidade do modelo em descrever o comportamento livre de escala da distribuição de graus, ele não comtempla a diversidade de expoentes determinados empiricamente em diversas redes reais, com expoentes variando num amplo intervalo ao redor de $3(1 \leq \gamma \leq 4)$, limitando sua aplicação $[6,10]$. Esta limitação tem motivado a comunidade científica no sentido de generalizar o modelo de diversas maneiras, tais como a redefinição da probabilidade de conexão como sendo proporcional a $k+k_{0}$, onde $k_{0}$ é uma constante positiva, conduzindo a bons resultados, com o expoente variando no intervalo faixa $2<\gamma<\infty[6,10]$.

\subsection{Propagação de Opiniões e a Estatística de E- leições Proporcionais}

Nesta seção apresentaremos algumas informações sobre propriedades estatísticas de eleições proporcionais e alguns dos modelos mais estudados na modelagem do processo de formação e propagação de opiniões em uma comunidade.

\subsubsection{Comportamento Estatístico de Eleições Proporcionais}

Em 1999 Costa-Filho e colaboradores [3] notaram uma curiosa propriedade estatística na distribuição dos votos recebidos pelos diversos candidatos em eleições proporcionais brasileiras. O comportamento era o mesmo, embora o ano e os colégios eleitorais mudassem. De acordo 
com este estudo, o número de candidatos com $v$ votos obedecia uma lei de potências na forma

$$
N(v)=v^{-\alpha}
$$

$\operatorname{com} \alpha \approx-1$. Essa mesma relação foi confirmada por Fábio Vannucchi para eleições municipais em São Paulo [6], com cuidadoso ajuste nos dados estatísticos fornecedos pelo TRE. Embora o expoente mudasse ligeiramente, a distribuição de votos continuava obedecendo uma lei de potências.

Essa mesma propriedade foi observada ainda por Gonzáles e colaboradores para a Câmara Baixa de alguns Estados da Índia [17], o que indica que ela é robusta, sugerindo que há algo mais fundamental por trás desse comportamento (como os mecanismos intrínsecos de convencimento), atuando de forma relativamente independente da cultura ou de leis eleitorais.

Isso sugere que o uso de modelos relativamente simples possam ser úteis na identificação dos ingredientes fundamentais para que esse comportamento seja observado.

\subsubsection{Modelo de Ising com Dinâmica de Glauber}

Nas últimas décadas, os físicos começaram a explorar ativamente a dinâmica de opiniões e muitos modelos foram concebidos [2]. Em geral, os modelos são inspirados em dinâmicas para o modelo de Ising da Mecânica Estatística de Não Equilíbrio, como a dinâmica de Glauber $[2]$.

A proposta de Glauber consiste em assumir uma equação mestra e formas plausíveis para as taxas de transição entre os estados do sistema de spins que interagem segundo o modelo de Ising [18, 19]. A escolha das taxas de transições é feita exigindo-se que a condição de 
balaceamento detalhado seja obedecida, de maneira que a dinâmica se reduza ao modelo de Ising no equilíbrio [18, 19].

Assim, evolução temporal do sistema no caso unidimensional é governada pela equação mestra $[18,19]$

$$
\frac{\partial}{\partial t} P(\sigma, t)=\sum_{\sigma^{\prime} \neq \sigma}\left[W_{\sigma^{\prime} \rightarrow \sigma} P\left(\sigma^{\prime}, t\right)-W_{\sigma \rightarrow \sigma^{\prime}} P(\sigma, t)\right],
$$

onde $P(\sigma, t)$ é a probabilidade de ocorrência da configuração $\{\sigma\}$ no instante t, $W_{\sigma \rightarrow \sigma^{\prime}}$ é a taxa de transição entre os estados com configurações $\{\sigma\}$ e $\left\{\sigma^{\prime}\right\}$.

A cada instante, são consideradas apenas as transições entre configurações que diferem por apenas um spin, ou seja, uma dinâmica sequencial. Portanto, a taxa de transição é escrita como [18]

$$
W_{\sigma \rightarrow \sigma^{\prime}}=\sum_{i=1}^{N} \delta_{\sigma_{1}, \sigma_{1}^{\prime}} \delta_{\sigma_{2}, \sigma_{2}^{\prime}} \ldots \delta_{\sigma_{i}, \sigma_{i}^{\prime}} \ldots \delta_{\sigma_{N}, \sigma_{N}^{\prime}} w_{i}(\sigma)
$$

onde $\delta_{\sigma_{i}, \sigma_{i}^{\prime}}$ é o símbolo delta de Kronecker, ou seja,

$$
\delta_{\sigma_{j}, \sigma_{j}^{\prime}}=\left\{\begin{array}{lll}
1 & \text { se } & \sigma_{j}=\sigma_{j}^{\prime} \\
0 & \text { se } & \sigma_{j} \neq \sigma_{j}^{\prime}
\end{array}\right.
$$

O fator $w_{i}(\sigma)$ é a taxa de inversão do i-ésimo spin de $\{\sigma\}$, ou seja, $\sigma_{i} \rightarrow-\sigma_{i}$. Denotaremos por $\left\{\sigma^{i}\right\}=\left(\sigma_{1}, \sigma_{2}, \ldots,-\sigma_{i}, \ldots, \sigma_{N}\right)$ a configuração resultante da inversão.

Com esta taxa de transição, a equação mestra passa a ser dada por

$$
\frac{\partial}{\partial t} P(\sigma, t)=\sum_{i=1}^{N}\left[w_{i}\left(\sigma^{i}\right) P\left(\sigma^{i}, t\right)-w_{i}(\sigma) P(\sigma, t)\right]
$$

A correspondência com o modelo de Ising em 1D é feita escolhendose a taxa de transição como

$$
w_{i}\left(\sigma_{i}\right)=\frac{1}{2} \alpha\left[1-\frac{1}{2} \gamma \sigma_{i} \sum_{j \in \Gamma_{i}} \sigma_{j}\right]
$$


onde $\Gamma_{i}$ é a vizinhança do vértice $i, \gamma$ descreve a tendência de alinhamento entre sítios vizinhos e $\alpha$ define a escala de tempo em que ocorre a inversão de um spin [18].

Explicitamente, existem três possibilidades para a taxa de transição, a saber [18],

$$
w_{i}\left(\sigma_{i}\right)=\left\{\begin{array}{c}
\frac{1}{2} \alpha(1-\gamma) \\
\frac{1}{2} \alpha \\
\frac{1}{2} \alpha(1+\gamma)
\end{array}\right.
$$

O primeiro valor corresponde ao caso em que o i-ésimo spin é paralelo aos seus vizinhos, o segundo corresponde ao caso em que os vizinhos são antiparalelos entre si e o terceiro corresponde ao caso em que o i-ésimo spin é antiparalelo aos seus vizinhos. Se $\gamma>0$, o alinhamento entre os spins é favorecido e o modelo descreve o ferromagnetismo, caso contrário, o modelo descreve antiferromagnetismo [18].

Se impusermos ainda que o estado de equilíbrio seja caracterizado pela distribuição de Gibbs (em 1D), obtemos [18]:

$$
\begin{aligned}
& \gamma=\tanh (2 \beta J) \\
& w_{i}\left(\sigma_{i}\right)=\frac{1}{2} \alpha\left[1-\sigma_{i} \tanh \left(\beta J \sum_{j \in \Gamma_{i}} \sigma_{j}\right)\right]
\end{aligned}
$$

No caso particular de temperatura nula:

$$
\begin{aligned}
& \gamma \rightarrow 1 \\
& w_{i}\left(\sigma_{i}\right) \rightarrow\left\{\begin{array}{c}
0 \\
\frac{1}{2} \alpha \\
\alpha
\end{array}\right.
\end{aligned}
$$

Do ponto de vista analítico, podemos ainda construir um algoritmo numérico do modelo como segue [18, 19]:

1. Discretizamos o tempo em intervalos $\Delta t$ e definimos a taxa de transição de $\{\sigma\}$ para $\left\{\sigma^{\prime}\right\}$ como $T_{\sigma \rightarrow \sigma^{\prime}}=W_{\sigma \rightarrow \sigma^{\prime}} \Delta t$ 
2. Escolhemos $\Delta t=\frac{1}{\alpha N}$ de maneira que

$$
\begin{aligned}
& T_{\sigma \rightarrow \sigma^{\prime}}=\frac{1}{N} \sum_{i=1}^{N} \delta_{\sigma_{1}, \sigma_{1}^{\prime}} \delta_{\sigma_{2}, \sigma_{2}^{\prime}} \ldots \delta_{\sigma_{i}, \sigma_{i}^{\prime}} \ldots \delta_{\sigma_{N}, \sigma_{N}^{\prime}} \tilde{w}_{i}(\sigma) \\
& \tilde{w}_{i}(\sigma)=\frac{1}{2}\left[1-\sigma_{i} \tanh \left(\beta J \sum_{j \in \Gamma_{i}} \sigma_{j}\right)\right]
\end{aligned}
$$

3. A partir de uma configuração inicial $\left\{\sigma_{0}\right\}=\left(\sigma_{1}^{0}, \ldots, \sigma_{N}^{0}\right)$ sorteamos um número aleatório $\xi$ uniformemente entre 0 e 1 .

4. A i-ésima variável de spin é alterada de acordo com

$$
\left\{\begin{array}{lll}
\text { se } \xi \leq \tilde{w}_{i}\left(\sigma_{i}\right) & \text { então } & \sigma_{i} \rightarrow-\sigma_{i} \\
\text { se } \xi>\tilde{w}_{i}\left(\sigma_{i}\right) & \text { então } & \sigma_{i} \rightarrow \sigma_{i}
\end{array}\right.
$$

Para tempos longos as configurações serão escolhidas de acordo com a distribuição de Gibbs, satisfazendo o equilíbrio [18, 19].

Dentre os resultados da aplicação da dinâmica de Glauber, temos que, a temperatura nula e em uma dimensão, ela sempre evolui para uma configuração ordenada, ou seja, após algum tempo todos os sítios se encontram num mesmo estado, seja ele $\sigma=1$ ou $\sigma=-1$ de acordo com o resultado esperado para o modelo de Ising $[6,18]$. Para dimensões superiores, existe uma probabilidade não nula de que configurações diferentes do estado ordenado ocorram. Estas configurações apresentam aglomerados de sítios com o mesmo estado formando domínios magnéticos [6, 18].

Os domínios magnéticos podem ser caracterizados analiticamente por um parâmetro de ordem denominado densidade de interfaces definido por $[6]$

$$
\rho(t)=\frac{1}{2 A} \sum_{i=1}^{N} \sum_{j \in \Gamma_{i}} \frac{1}{2}\left(1-\sigma_{i}(t) \sigma_{j}(t)\right)
$$


onde $k_{i}$ é o grau de um vértice $i$ da rede, $\Gamma_{i}$ é a vizinhança do vértice $i, A=\frac{1}{2} \sum_{i} k_{i}$ é o número total de arestas da rede e t é um instante particular da dinâmica.

No caso unidimensional, a densidade média de interface à temperatura nula apresenta comportamento assintótico como lei de potências do tipo $\langle\rho(t)\rangle \sim t^{-\frac{1}{2}}$, confirmando o fato do modelo sempre apresentar configurações ordenadas no equilíbrio [6].

O modelo Ising-Glauber notabiliza-se por ser um dos poucos modelos dinâmicos em que é possível calcular variáveis estocásticas de forma exata. Diante das limitações encontradas para uma formulação geral de fenômenos fora do equilíbrio, o modelo é uma alternativa adequada e suficientemente simples para a realização dos cálculos [18, 19].

\subsubsection{Modelo da Regra da Maioria}

Motivados pela dinâmica de Glauber, podemos fazer uma analogia entre o sistema de spin $\frac{1}{2}$ e uma comunidade de indivíduos que possuem uma opinião binária sobre um determinado assunto, colocando-se de maneira favorável ou contra o tema em debate, e, à medida que o tempo passa, mudam de opinião de acordo com o que pensam seus vizinhos $[2,18]$.

O Modelo da Regra da Maioria propõe que, a cada iteração, um grupo de $r$ indivíduos seja escolhido aleatoriamente simulando um grupo de discussão, e, como consequência desta discussão, todos os integrantes do grupo passam a assumir a opinião da maioria $[2,18]$.

O tamanho do grupo não é necessariamente fixado, podendo obedecer a uma determinada distribuição a cada iteração [2]. Se $r$ é ímpar, a maioria estará bem definida, entretanto, se $r$ é par, existe a possibilidade de ausência de maioria (ambas as opiniões são assumidas por cada metade do grupo). Neste caso, introduzimos uma predisposição 
em favor de uma das opiniões, digamos, a opinião $\sigma=+1$ prevalece [2]. Esta prescricão remonta o princípio da inércia social, no qual as pessoas tendem a serem conservadoras [2].

Dentre os resultados do modelo, destacamos a capacidade em reproduzir uma transição de fases entre os dois estados de consenso dependendo da distribuição inicial de opiniões pela rede. Um simples cálculo de campo médio permite verificar este resultado, como segue [2].

Consideremos $p_{+}$como a fração de sítios com opinião $+1, p_{-}=$ $1-p_{+}$como a fração de sítios com opinião -1 e $p_{+}^{0}$ como a fração inicial de indivíduos com opinião +1 . Por simplicidade, consideremos ainda que $r$ é constante e igual a 3 e a rede de contatos como sendo o grafo completo ${ }^{8}$ [2]. Assim, a variação de $N_{+}$, obedece a equação [2]

$$
\Delta N_{+}=3\left(p_{+}^{2} p_{-}-p_{+} p_{-}^{2}\right) \Rightarrow \frac{\Delta N_{+}}{N} \frac{N}{3}=\left(p_{+}^{2} p_{-}-p_{+} p_{-}^{2}\right)
$$

Se definirmos $\frac{1}{\Delta t}=\frac{N}{3}$ e tomarmos o limite termodinâmico:

$$
\dot{p}_{+}=-2 p_{+}\left(p_{+}-\frac{1}{2}\right)\left(p_{+}-1\right)
$$

Os pontos fixos de 2.17 são imediatos:

$$
p_{+}^{*}=\left\{\begin{array}{c}
0 \\
\frac{1}{2} \\
1
\end{array}\right.
$$

Evidentemente, os pontos fixos $p_{+}^{*}=0$ e $p_{+}^{*}=1$ são estáveis, enquanto que o ponto $p_{+}^{*}=\frac{1}{2}$ é instável e é interpretado fisicamente como o ponto crítico da dinâmica. Além disso, $p_{+}^{*}=p_{c}=\frac{1}{2}$ define a separação entre as bacias de atração do sistema no espaço das probabilidades [2]:

$$
\left\{\begin{array}{l}
p_{+}^{0}<p_{c} \Rightarrow p_{+}(t) \rightarrow p_{+}^{*}=0 \\
p_{+}^{0}>p_{c} \Rightarrow p_{+}(t) \rightarrow p_{+}^{*}=1
\end{array}\right.
$$

\footnotetext{
${ }^{8} \mathrm{~A}$ paridade de $r$ foi escolhida de maneira a manter a simetria entre as duas opiniões, evitando a aplicação do princípio da inércia social.
} 
Em geral, cálculos analíticos para este modelo em situações mais gerais (ou mesmo em redes regulares) apresentam sérias dificuldades, impedindo sua solução analítica [2].

Modificações do Modelo da Regra da Maioria incluem, por exemplo, difusão de cabos eleitorais, número variável de vizinhos (redes dinâmicas), a introdução de uma opinião particular tendenciosa, a introdução de opositores e a introdução de inomogeneidades na rede (presença de sítios inflexíveis quanto ao seu estado) [2]. A seguir, apresentaremos dois interessantes modelos resultantes de modificações do Modelo da Regra da Maioria, o Modelo do Votante Majoritário e o Modelo do Votante.

\subsubsection{Modelo do Votante Majoritário}

O Modelo do Votante Majoritário é uma dinâmica sequêncial em que, a cada instante, um vértice da rede assume o estado da maioria de seus vizinhos com probabilidade $1-q$ e, com probabilidade q, assume o estado da minoria. Se não houver maioria, o estado inverte com

probabilidade $\frac{1}{2}$ [18]. Ou seja, a dinâmica é governada pela mesma equação mestra do Modelo de Glauber (2.9) com taxa de inversão dada por $[18]$

$$
w_{i}\left(\sigma_{i}\right)=\frac{1}{2}\left[1+(2 q-1) \sigma_{i} \operatorname{sgn}\left(\sum_{j \in \Gamma_{i}} \sigma_{j}\right)\right]=\left\{\begin{array}{c}
q \\
\frac{1}{2} \\
1-q
\end{array}\right.
$$

Em uma dimensão o Modelo do Votante Majoritário coincide com o Modelo de Glauber à Temperatura Nula [18].

Com este modelo, estamos diante de uma comunidade de indivíduos hesitantes, isto é, indivíduos que às vezes agem contrariamente a opinião da maioria dos seus vizinhos [18]. O parâmetro $q$ mede o grau de 
hesitação dos indivíduos e é interpretado como um ruído à dinâmica (temperatura) [18].

Em redes regulares em duas ou mais dimensões, o modelo apresenta transição de fase entre uma fase ferromagnética e paramagnética em função do parâmetro $q$ [18]. Resultados de simulações numéricas indicam que seu comportamento crítico é o mesmo do modelo IsingGlauber, o que nos leva a afirmar que ambos estão na mesma classe de universalidade [18]. Este modelo também apresenta transição de fase do tipo ordem-desordem em grafos aleatórios e redes de mundo pequeno [18].

\subsubsection{Modelo do Votante}

Neste modelo, a cada passo de tempo, um determinado sítio escolhe aleatoriamente um de seus vizinhos e passa a ter a opinião dele. Desta forma, o sítio passa a ter uma das opiniões com probabilidades proporcionais ao número de vizinhos que compartilham dela [18].

A evolução temporal desse modelo é governada por (2.9) com taxa de inversão dada por [18]

$$
w_{i}\left(\sigma_{i}\right)=\frac{1}{2}\left[1-\sigma_{i}\left(\frac{1}{k_{i}} \sum_{j \in \Gamma_{i}} \sigma_{j}\right)\right]
$$

Mais uma vez, este modelo coincide com a dinâmica de Glauber quando ambos são considerados em uma dimensão.

É imediato que as configurações de consenso são absorventes pois $w_{i}\left(\sigma_{i}=+1, \forall i\right)=w_{i}\left(\sigma_{i}=-1, \forall i\right)=0^{9}$. Em reticulados de dimensões superiores o modelo pode atingir outros estados de consenso, sendo que em redes de tamanho infinito existe a possibilidade do sistema nunca se ordenar [6]. Numa série de redes distintas esse comportamento é

\footnotetext{
${ }^{9}$ Estado absorvente é aquele que pode ser atingido a partir de outros estados, mas a recíproca é proibida. Uma vez que o sistema atige seu estado absorvente a dinâmica cessa.
} 
mantido sendo que, no limite termodinâmico, a capacidade do sistema em atingir configurações ordenadas depende, essencialmente, do grau médio da rede [6].

Todos os modelos apresentados até agora possuem uma importante propriedade: a influência flui da vizinhança para o sítio a ser atualizado, ou, coloquialmente, a influência flui de fora para dentro. Diferentemente destas versões, no Modelo Sznajd, a ser apresentado na próxima seção, a influência apresenta a peculiar propriedade de fluir do sítio em consideração para sua vizinhança.

\subsection{O Modelo Sznajd e suas Variantes}

Nesta seção, apresentaremos um modelo bastante empregado na modelagem de opiniões na última década e que será o objeto de estudo desta dissertação, o Modelo Sznajd. Seu sucesso deve-se, dentre outros aspectos, à capacidade de descrever os resultados apontados por CostaFilho, relacionados ao comportamento universal observado em eleições proporcionais $[6,20]$.

\subsubsection{O Modelo Sznajd}

Em 2 000, Katarzina Sznajd-Weron e Jósef Sznajd propuseram um modelo denominado USDF (United we Stand, Divided we Fall) que ganhou destaque na última década por sua grande simplicidade e capacidade de descrever comportamentos universais de eleições proporcionais e majoritárias [1]. Tornou-se um modelo bastante estudado no contexto da evolução de opiniões por representar uma situação em que, diferentemente da dinâmica de Glauber e do modelo do votante, a opinião se propaga de dentro para fora, ou seja, a partir de pares de vizinhos com a mesma opinião, o que parece ser muito adequado 
para representar certos aspectos da realidade dos processos de convencimento [20].

O Modelo Sznajd é caracterizado por um conjunto de regras simples para a interação entre vizinhos que, a partir de uma configuração aleatória, permite a formação de grandes domínios de sítios no mesmo estado (ou seja, com a mesma opinião), até que, para tempos suficientemente longos, um único domínio compreende toda a rede, caracterizando um estado estacionário de consenso [1, 20].

A versão original do modelo considerava uma cadeia com condições periódicas de contorno onde cada membro pode assumir apenas dois estados (opiniões) $\sigma= \pm 1$ com a seguinte dinâmica [1]:

1. Se $\sigma(i, t) \sigma(i+1, t)=1$, então $\sigma(i-1, t+1)=\sigma(i+2, t+1)=\sigma(i, t)$.

2. Se $\sigma(i, t) \sigma(i+1, t)=-1$, então $\sigma(i-1, t+1)=\sigma(i+1, t) \mathrm{e}$ $\sigma(i+2, t+1)=\sigma(i, t)$.

Assim, se um par de sítios adjacentes compartilham a mesma opinião, eles impõem esta para seus vizinhos. Se, ao contrário, o par é discordante, cada um deles impõe sua opinião ao vizinho do outro. As opiniões são atualizadas numa ordem aleatória, iniciando-se de uma configuração inicial também aleatória onde as opiniões são igualmente distribuídas [2]. Uma comunidade fechada com tais regras poderia modelar, por exemplo, eleições presidenciais em uma sistema bipartidário [1].

Este modelo sempre leva a um de três estados estacionários [1, 2]:

1. Dois estados que podem ser chamados de 'ferromagnéticos' (todos os sítios com a mesma opinião, $\sigma=1$ ou $\sigma=-1$ ), no qual a comunidade atinge o consenso.

2. Um estado que pode ser considerado 'antiferromagnético' no qual cada candidato tem $50 \%$ dos votos e tem opiniões intercaladas. 
Este estado é possível apenas para uma cadeia com um número par de sítios.

Este último estado é consequência da regra (2) que favorece o dissenso.

O modelo USDF, renomeado posteriormente por Stauffer como Modelo Sznajd [21], tem sido modificado e aplicado nos mais variados contextos socio-físicos, como por exemplo, em marketing [22, 23], mercado financeiro [24] e política [5, 25], sugerindo novas aplicações para a Mecânica Estatística [20].

\subsubsection{Modificações do Modelo Sznajd}

$\mathrm{Na}$ tentativa de incluir características mais realistas e evitar o consenso como único estado estacionário possível, várias modificações foram propostas ao modelo USDF [2]. Nestas versões do modelo, uma ou mais regras são alteradas a fim de incluir novos ingredientes como a possibilidade de mais de duas opiniões, difusão de cabos eleitorais, redução na capacidade de convencimento dos agentes ou diferenças de topologia na rede de modo a redefinir o relacionamento entre os eleitores $[2,4]$.

Nas primeiras versões modificadas do modelo, algumas publicações preocuparam-se em evitar o estado antiferromagnético por considerarem pouco realista. Nas versões alternativas mais comuns, apenas a regra ferromagnética é mantida, de maneira que vizinhos de um par discordante mantém suas opiniões inalteradas, ou seja, a regra antiferromagnética de propagação da divergência foi abandonada [1, 26].

Outro fato relevante para a busca de melhorias no modelo devese a publicação de Behera e Schweitzer [27], mostrando que a direção do fluxo de informação é irrelevante em modelos unidimensionais e, consequentemente, que a dinâmica do Modelo Sznajd é equivalente a dinâmica do Modelo do Votante em 1D. 
Além disso, um modelo unidimensional não deve descrever adequadamente todas as propriedades de sistemas sociais reais e generalizações para dimensões superiores são necessárias [2]. Generalizações para redes quadradas foram exploradas por Stauffer e colaboradores $[2$, 21]. Nestes trabalhos, o modelo é construído sobre uma rede quadrada $L \mathrm{x} L$ onde, novamente, cada sítio pode assumir dois estados e seis diferentes regras são adicionadas. Entretanto, apenas uma destas regras se perpetuou ao longo de publicações posteriores, a saber, um par de sítios afeta a opinião de seus seis primeiros vizinhos apenas se concordarem entre si [21].

Considerando estas modificações, o estado de consenso é sempre alcançado como estado estacionário. Se a magnetização inicial é negativa $(m<0)$, o sistema sempre alcança o consenso com $m=-1$, entretanto, se a magnetização inicial é positiva, o consenso é alcançado com $m=1^{10}[21]$.

Um próximo passo em direção a um maior realismo seria a investigação do modelo em redes complexas [7]. Muitas publicações apresentam estudos em diversas topologias como grafos completos [28], redes mundo-pequeno [29] e redes livres de escala [5, 17] com diversas contribuições analíticas e numéricas [20]. A mudança da topologia confere maior riqueza e fidelidade na descrição das relações sociais que podem ser estabelecidas entre os indivíduos de uma comunidade. A complexidade das relações humanas ainda podem ser consideradas com o aumento do alcance das interações e do número de variáveis de estado, a inclusão de ruído ou difusão [20].

É interessante considerarmos também a possibilidade dos sítios assumirem mais que duas opiniões (como no modelo de Potts). Isto é particularmente importante na aplicação do modelo à política, ao des-

\footnotetext{
${ }^{10}$ Se a magnetização inicial é nula, podemos afirmar que, por simetria, os estados $m=1$ e $m=-1$ podem ser atingidos com probabilidade $\frac{1}{2}$ para cada um deles.
} 
crever eleições proporcionais com $M>2$ candidatos [5, 25]. Nesta adaptação, cada sítio pode assumir $\mathrm{M}+1$ estados, sendo o estado extra associado à indecisão do eleitor.

Estas modificações no modelo permitem reproduzir, durante o transiente, a lei de potências observada na distribuição de votos por candidatos em eleições realizadas no Brasil e na Índia [3, 17]. É importante enfatizarmos que, para estabelecermos um contato com a questão eleitoral, é necessário analisarmos o modelo em seu transiente, pois uma eleição ocorre após um tempo relativamente curto de campanha, truncando-se a evolução temporal da opinião dos eleitores. Em geral, supõe-se que o tempo de campanha é insuficiente para se estabelecer um estado estacionário no sistema.

Diante da grande quantidade de publicações que exploram variantes do Modelo Sznajd, resolvemos adotar neste trabalho a versão proposta por Bernardes e colaboradores em que o modelo é generalizado para uma rede complexa com mais de duas opiniões, com o objetivo de explorar propriedades dos estados estacionários e correlacioná-las com aplicações em eleições proporcionais [5]. Nos próximos capítulos analisaremos em detalhe este modelo através de uma aproximação de campo médio e de abordagens numéricas.

\subsubsection{O Modelo Sznajd Complexo}

Em 2001 Bernardes e colaboradores [25] consideraram o modelo Sznajd em uma rede quadrada com $M>2$ candidatos. A distribuição observada por Costa-Filho é reproduzida em seu transiente mas o resultado é obtido apenas para condições iniciais bastante específicas, dificultando a compreenção da origem de sua robustez e portanto, a validação do modelo.

Diante desta dificuldade, Bernardes e colaboradores [5] adaptaram o 
modelo para uma rede de Barabási-Albert e mostraram que o transiente do modelo, neste caso, apresenta o comportamento estatístico esperado independentemente da condição inicial considerada. Além disso, a topologia das redes complexas é muito mais próxima da realidade do que a topologia de redes regulares, tornando esta nova abordagem mais razoável.

Para efetivarem sua adaptação, eles consideraram que, em média, cada sítio convence um vizinho por interação, de forma a preservar as características do modelo original, definindo a probabilidade de convencimento do sítio i como o inverso de sua conectividade $\left(p_{i}=\frac{1}{k_{i}}\right)$ $[5]$.

Nesta reformulação do modelo em redes complexas, que denominaremos Modelo de Sznajd Complexo (MSC), a cada sítio está associado, em cada instante $\mathrm{t}$, um valor inteiro $\sigma(i, t) \in\left\{0,1,2, \ldots, N_{c}\right\}$ que representa a opinião assumida pelo eleitor. O estado $\sigma=0$ representa um eleitor indeciso, ou seja, que ainda não tem uma opinião formada, enquanto que os demais candidatos são enumerados de 1 a $N_{c}$ e representam as diferentes opiniões que os eleitores podem assumir.

Geralmente as condições iniciais da rede são padronizadas, de maneira que todos os eleitores estão indecisos, a menos de $N_{c}$ eleitores escolhidos aleatoriamente votando em cada um dos candidatos. Esse processo é denominado semeadura da rede.

A dinâmica de convencimento consiste na escolha, a cada passo, de um sítio $i$ da rede e aplicando o seguinte conjunto de regras $[4,6,30]$ :

(I) Caso o eleitor escolhido i não seja indeciso $(\sigma(i, t) \neq 0)$, um de seus vizinhos $\mathrm{j}\left(j \in \Gamma_{i}\right)$ é sorteado e a regra (II) é aplicada; caso contrário, nada ocorre.

(IIa) Se o eleitor j está indeciso $(\sigma(j, t)=0)$, então $\mathrm{j}$ pode ser convencido por i com probabilidade igual ao inverso do grau de i 
$\left(p_{i}=\frac{1}{k_{i}}\right)$.

(IIb) Se ambos i e j compartilham da mesma opinião, então i e j tentam convencer cada um de seus respectivos vizinhos com probabilidades iguais ao inverso de seus respectivos graus.

(IIc) Se i e j não compartilham da mesma opinião, nada ocorre.

O processo é repetido até que todos os sítios da rede tenham sido sorteados uma vez (a ordem é aleatória).

Tudo isso pode ser resumido na Tabela 2.1.

\begin{tabular}{|c|c|c|c|}
\hline$\sigma(i, t)$ & $\sigma(j, t)$ & - & Resultado \\
\hline$=0$ & - & & Nada ocorre \\
\hline \multirow{3}{*}{$\neq 0$} & $=0$ & & $\sigma(j, t+1)=\sigma(i, t)$ \\
\cline { 2 - 4 } & $\neq 0$ & $\sigma(j, t)=\sigma(i, t)$ & Assediam seus respectivos vizinhos \\
\cline { 3 - 4 } & & $\sigma(j, t) \neq \sigma(i, t)$ & Nada ocorre \\
\hline
\end{tabular}

Tabela 2.1: Tabela resumindo as etapas da dinâmica de convencimentos do Modelo Sznajd Complexo.

Neste trabalho, além das regras convencionais, estamos particularmente interessados em estudar a influência de um campo externo (ruído) na dinâmica do modelo. Isso é feito com a inclusão de uma terceira regra, apresentada a seguir [6].

(III) O eleitor i pode escolher um candidato aleatoriamente com probabilidade $w$ ou seguir as regras convencionais com probabilidade complementar $1-w$.

A Tabela 2.2 destaca a inclusão da regra associada ao campo externo. 


\begin{tabular}{|c|c|c|c|c|}
\hline Ruído & $\sigma(i, t)$ & $\sigma(j, t)$ & - & Resultado \\
\hline \multirow{3}{*}{$1-w$} & $=0$ & - & & Nada ocorre \\
\cline { 2 - 5 } & \multirow{3}{*}{$\neq 0$} & $=0$ & & $\sigma(j, t+1)=\sigma(i, t)$ \\
\cline { 3 - 5 } & & $\neq 0$ & $\sigma(j, t)=\sigma(i, t)$ & Assediam seus vizinhos \\
\cline { 4 - 5 } & & & $\sigma(j, t) \neq \sigma(i, t)$ & Nada ocorre \\
\hline$w$ & - & - & & Candidato aleatório \\
\hline
\end{tabular}

Tabela 2.2: Tabela resumindo as etapas da dinâmica de convencimentos do Modelo Sznajd Complexo com a inclusão do ruído.

A aplicação do campo externo pretende representar a interferência de campanhas publicitárias na opinião do eleitorado. Numa linguagem física, podemos interpretar o campo aplicado como a interação do sistema com um banho térmico que origina flutuações não locais na opinião dos eleitores (ruído). Desta forma, com probabilidade $w$, o eleitor não aceita o confroto de idéias com seus vizinhos e prefere escolher um candidato que o assedia através de uma propaganda.

Como no modelo de Sznajd original, o Modelo Sznajd Complexo sem Ruído sempre apresenta um estado absorvente de consenso [4, 6]. Os estados estacionários do Modelo Sznajd Complexo serão discutidos em detalhes nos capítulos 3 e 4 .

\subsubsection{Regras de Confiança Limitada - O Modelo Sznajd Ge- neralizado}

Nas versões do Modelo Sznajd abordadas anteriormente, um sítio sempre é capaz de convencer outro independentemente de sua opinião, de maneira que o sistema sempre evolui para um estado absorvente de consenso. Na prática, pode-se pensar que indivíduos de uma comunidade se influenciam apenas quando suas opiniões são suficientemente próximas, afinal, é pouco provável convencer uma pessoa de algo muito 
diferente do conjunto de suas convicções. Além disso, é fato a existência de diferentes facções em comunidades, com estados estacionários de coexistência.

A introdução de uma regra conhecida como Confiança Limitada (Bounded Confidence) permite modelar tal situação. Com esta regra, dois sítios só podem interagir se suas opiniões forem suficientemente próximas.

Em 2 000, Defuant e colaboradores [31] introduziram esta idéia para modelos com opiniões continuas $(\sigma \in[0,1])$ impondo a restrição de que $\sigma_{i}$ e $\sigma_{j}$ interagem apenas se $\left|\sigma_{i}-\sigma_{j}\right| \leq \epsilon$. Dentre os resultados obtidos está o fato de que o modelo apresenta, além do estado de consenso, outros estados estacionários onde é possível a coexistência de duas ou mais opiniões que não interagem mutuamente.

Em um modelo em que as opiniões são discretas, como o Modelo Sznajd, esta modificação é normalmente adaptada de forma que as opiniões são enumeradas de 0 a $\mathrm{M}$ e se um sítio tem opinião $\sigma$, ele só pode convencer e ser convencido por sítios que tenham opinões $\sigma-1$ e $\sigma+1(\epsilon=1)$ [4]. Esta modificação é capaz de gerar um estado estacionário com coexistência de opiniões, mas não permite uma transição gradual entre confiança total (as opiniões $\sigma-1$ e $\sigma+1$ sempre podem convencer o sítio com opinião $\sigma$ ) e total falta de confiança (as outras opiniões nunca podem convencer o sítio com opinião $\sigma$ ) [4].

Na tentativa de analisar situações mais gerais, Timpanaro e Prado [4] propuseram um modelo que generaliza as regras propostas por Stauffer. O modelo foi nomeado Modelo Sznajd Generalizado. Este modelo inclui, em um único conjunto de regras, o Modelo Sznajd Original (USDF), O Modelo Sznajd Complexo, O Modelo Sznajd com Confiança Limitada proposta por Stauffer, bem como muitas outras possibilidades $[4]$.

No Modelo Sznajd Generalizado, há a imposição de que um sítio 
com opinião $\sigma^{\prime}$ tem a probabilidade $p_{\sigma^{\prime} \rightarrow \sigma}$ de ser convencido por outro sítio com opinião $\sigma$ [4]. Os diversos $p_{\sigma^{\prime} \rightarrow \sigma}$ são parâmetros do modelo e foram chamados genericamente de 'regras de confiança' [4]. Assim como nos modelos anteriores, a dinâmica de convencimento consiste, em cada instante, na escolha aleatória dos sítios da rede sujeitas ao seguinte conjunto de regras ${ }^{11}[4]$ :

(I') Escolhemos um eleitor i e um de seus vizinhos $j \in \Gamma_{i}$ aleatoriamente. Se $\sigma_{i} \neq \sigma_{j}$ nada ocorre; caso contrário aplicamos a regra (II').

(II') O sítio $j$ escolhe aleatoriamente um de seus vizinhos $j^{\prime}$ e tenta convencê-lo com probabilidade $p_{\sigma_{j}^{\prime} \rightarrow \sigma_{j}}$.

Perceba que não assumimos a priori nenhuma hipótese sobre a probabilidade de convencimento $p_{\sigma^{\prime} \rightarrow \sigma}$. No caso particular de $p_{\sigma^{\prime} \rightarrow \sigma}$ ser sempre igual a 0 ou 1, uma escolha apropriada dos valores permite-nos recuperar tanto o Modelo USDF como o Modelo Sznajd com Confiança Limitada de Stauffer.

De maneira geral, Timpanaro e Prado [4] caracterizam as condições necessárias para que haja coexistência de opiniões no estado estacionário para hipóteses muito gerais quanto às probabilidades $p_{\sigma^{\prime} \rightarrow \sigma}$, generalizando os resultados de Defuant e colaboradores. Por exemplo, se considerarmos $p_{\sigma^{\prime} \rightarrow \sigma} \neq 0, \forall \sigma, \sigma^{\prime}$; apenas os estados de consenso possuirão bacias de atração com um ponto repulsor na região do espaço de fase em que as $\mathrm{M}$ opiniões poderiam coexistir [4]. No caso particular $\mathrm{M}=3$, Timpanaro e Prado argumentam que esse repulsor deve ser sempre um ponto fixo instável, impedindo a coexistência de opiniões $[4]$.

\footnotetext{
${ }^{11}$ Por simplicidade, o modelo desconsidera a presença de eleitores indecisos. Não há perda de generalidade pois, em todos os casos abordados anteriormente, as regras da dinâmica implicavam em um decrescimento do número de indecisos a zero
} 


\subsubsection{Aproximação de Campo Médio em Sistemas Dinâmi- $\cos$}

Uma abordagem através de equações mestras é muito eficiente no tratamento da dinâmica do Modelo Sznajd, pois podemos aplicar a ela diversas técnicas desenvolvidas no estudo de sistemas dinâmicos.

A equação mestra que descreve o modelo é, genericamente,

$$
\begin{aligned}
\Delta \eta^{\sigma} & =\eta^{\sigma}(i, t+1)-\eta^{\sigma}(i, t) \\
& =\sum_{\sigma^{\prime} \neq \sigma}\left\{w_{\sigma^{\prime} \rightarrow \sigma} P\left(\sigma^{\prime}, t\right)-w_{\sigma \rightarrow \sigma^{\prime}} P(\sigma, t)\right\},
\end{aligned}
$$

onde $\eta^{\sigma}(i, t)$ é a probabilidade do sítio i votar no candidato $\sigma$ no instante t, $P(\sigma, t)$ é a probabilidade de ocorrência de uma configuração onde $\sigma(i, t)=\sigma$ e $w_{\sigma \rightarrow \sigma^{\prime}}$ é a taxa de transição entre os estados $\sigma$ e $\sigma^{\prime}$.

Fisicamente, $P\left(\sigma^{\prime} \rightarrow \sigma\right)=w_{\sigma^{\prime} \rightarrow \sigma} P\left(\sigma^{\prime}\right)$ corresponde a uma probabilidade de transição que favorece o candidato sigma, enquanto que $P\left(\sigma \rightarrow \sigma^{\prime}\right)=w_{\sigma \rightarrow \sigma^{\prime}} P(\sigma)$ corresponde a uma probabilidade que o desfavorece.

Os pontos fixos $\eta^{\sigma *}$ da dinâmica são determinados pela equação

$$
\Delta \eta^{\sigma}=0 \Rightarrow w_{\sigma^{\prime} \rightarrow \sigma} P\left(\sigma^{\prime}\right)=w_{\sigma \rightarrow \sigma^{\prime}} P(\sigma)
$$

e são identificados como os estados de equilíbrio do sistema.

A determinação dos pontos fixos é, em geral, impraticável, em virtude da alta complexidade matemática relacionada às taxas de transição e uma aproximação de campo médio deve ser feita.

Esperamos que, pelo menos na aproximação de campo médio, as taxas de transição e as probabilidades configuracionais sejam funções de $\eta^{\sigma}$, desta forma, a estabilidade dos pontos fixos pode ser obtida pela linearização do campo vetorial $F\left(\eta^{\sigma}\right)=\Delta \eta^{\sigma}\left(\eta^{\sigma}\right)$ associado ao sistema dinâmico autônomo nas vizinhanças do ponto fixo $\eta^{\sigma *}$ [32]. 
Uma expansão em série de potências para $\Delta \eta^{\sigma}$ nas vizinhanças do ponto fixo permite-nos escrever:

$$
\Delta \eta^{\sigma}=\Delta \eta^{\sigma}\left(\eta^{\sigma *}\right)+\left.\frac{\partial}{\partial \eta^{\sigma}}\left(\Delta \eta^{\sigma}\right)\right|_{\eta^{\sigma}=\eta^{\sigma *}}\left(\eta^{\sigma}-\eta^{\sigma *}\right)+O\left(\left(\eta^{\sigma}-\eta^{\sigma *}\right)^{2}\right)
$$

Nas proximidades do ponto fixo, podemos desprezar, com boa aproximação, os termos de ordem superior (estamos supondo tacitamente que o termo linear é não nulo). Além disso, temos que $\Delta \eta^{\sigma}\left(\eta^{\sigma *}\right)=0$, por definição. Assim:

$$
\Delta \eta^{\sigma}=A\left(\eta^{\sigma}-\eta^{\sigma *}\right), A=\left.\frac{\partial}{\partial \eta^{\sigma}}\left(\Delta \eta^{\sigma}\right)\right|_{\eta^{\sigma}=\eta^{\sigma *}}
$$

Portanto:

$$
\eta^{\sigma}(i, t+1)=(1+A) \eta^{\sigma}(i, t)-A \eta^{\sigma *}
$$

A solução geral de 2.23 é $[33,34]$ :

$$
\eta^{\sigma}(i, t)=\eta^{\sigma *}+C(1+A)^{t}, C \in \mathbb{R}
$$

O comportamento assintótico do tipo exponencial apresentado pela solução aproximada permite-nos inferir à respeito da estabilidade do ponto fixo. De fato, se $A>0$ ou $A<-2$, o ponto fixo é instável, pois as soluções se afastam exponencialmente dele, caso contrário, o ponto fixo é estável. Portanto, o critério de estabilidade é dado por [34]:

$$
\begin{gathered}
A>0 \text { ou } A<-2 \longrightarrow \text { Solução Instável. } \\
A<0 \text { ou } A>-2 \longrightarrow \text { Solução Estável. }
\end{gathered}
$$

Quanto à determinação dos pontos fixos, podemos ainda realizar uma série de inferências heurísticas. Primeiramente, esperamos que $\eta^{0}=0$ seja um ponto fixo estável da dinâmica pois as regras do modelo não permitem o crescimento do número de indecisos, implicando numa 
queda monotônica a zero com o tempo. De fato, podemos demonstrar este fato como segue: $\eta^{0}$ é, por construção, uma sequência monótona e limitada pelo intervalo $[0,1]$, portanto, $\lim _{t \rightarrow \infty} \eta^{0}(i, t)=\inf [0,1]=0$, ou seja, $\eta^{0} \rightarrow 0$ [35]. Esperamos ainda que o estado de consenso seja também um ponto fixo, em concordância com o Modelo de Sznajd original.

Em relação ao caso com ruído, é razoável esperar que, para intensos ruídos $(w \approx 1)$, a escolha aleatória de um candidato pelos eleitores predomine sobre a dinâmica convencional, possibilitando que um estado de equiprobabilidade entre os candidatos seja um terceiro ponto fixo da dinâmica.

Considerando todas estas expectativas, a introdução do ruído deve exibir uma transição de fases entre o estado de consenso e o estado de equiprobabilidade à medida que aumentamos a intensidade do ruído.

A seguir, apresentaremos um cálculo simples de campo médio que pretende verificar estas expectativas ${ }^{12}$. Um cálculo mais completo será realizado no próximo capítulo, onde exploraremos a aproximação de campo médio com mais detalhes.

De acordo com as regras de convecimento, podemos escrever para $\eta^{0}(i, t)$ :

$$
\begin{aligned}
\dot{\eta^{0}}= & (1-w)\left\{-\beta\left[\eta^{0} \eta^{1}+\eta^{0} \eta^{2}+\ldots+\eta^{0} \eta^{N_{c}}\right]-\right. \\
& \left.-\beta\left[\eta^{0}\left(\eta^{1}\right)^{2}+\eta^{0}\left(\eta^{2}\right)^{2}+\ldots+\eta^{0}\left(\eta^{N_{c}}\right)^{2}\right]\right\}+ \\
& +w\left\{-\beta^{\prime} N_{c} \eta^{0}\right\}
\end{aligned}
$$

É facil perceber que os termos precedidos pelo fator $(1-w)$ correspondem à dinâmica original, enquanto que o termo precedido pelo

\footnotetext{
${ }^{12}$ Por simplicidade, trataremos aqui o caso contínuo, que deve ser uma abordagem válida para o sistema no limite termodinâmico. Desta forma, é importante enfatizar que o critério de estabilidade dos pontos fixos passa a ser: soluções instáveis quando $A>0$ e soluções estáveis quando $A<0$.
} 
fator $w$ corresponde a interferência do ruído. Os fatores $\beta$ e $\beta^{\prime}$ estão associados às taxas de transição entre os estados e devem depender da geometria da rede considerada ${ }^{13}$.

Tendo em vista uma abordagem preliminar, apresentaremos a seguir a equação que descreve a variação na probabilidade de intenções de votos para o candidato $\sigma=1$. A equação para os demais candidatos é análoga e poupamos os detalhes.

$$
\begin{aligned}
\dot{\eta^{1}=} & (1-w)\left\{\beta\left[\eta^{0}\left(\eta^{1}\right)^{2}+\eta^{2}\left(\eta^{1}\right)^{2}+\ldots+\eta^{N_{c}}\left(\eta^{1}\right)^{2}\right]-\right. \\
& -\beta\left[\eta^{1}\left(\eta^{2}\right)^{2}+\eta^{1}\left(\eta^{3}\right)^{2}+\ldots+\eta^{1}\left(\eta^{N_{c}}\right)^{2}\right]+ \\
& \left.+\beta \eta^{0} \eta^{1}\right\}+ \\
& +w\left\{\beta^{\prime}\left[\eta^{0}+\eta^{2}+\ldots+\eta^{N_{c}}\right]-\beta^{\prime}\left(N_{c}-1\right) \eta^{1}\right\}
\end{aligned}
$$

Neste caso, a única diferença em relação ao caso dos indecisos é a presença de termos positivos que correspondem ao aumento nas intenções de votos do candidato $\sigma=1$.

Portanto, os pontos fixos são determinados pela resolução deste conjunto de $N_{c}+1$ equações diferenciais autônomas acopladas.

Em virtude do caráter de campo médio assumido na construção das equações, vamos propor algumas simplificações. No caso de $\dot{\eta}^{0}$, é aceitável considerarmos o comportamento médio de todos os demais candidatos sobre os eleitores indecisos. Desta forma, podemos escrever:

$$
\begin{aligned}
& \eta^{0}=x \\
& \eta^{1}=\eta^{2}=\ldots=\eta^{N_{c}}=y
\end{aligned}
$$

\footnotetext{
${ }^{13}$ No caso de campo médio, a dependência deve se dar através de médias dos parâmetros geométricas sobre toda a rede.
} 
A condição de normalização impõe $y=\frac{1-x}{N_{c}}$. Portanto:

$$
\begin{aligned}
\dot{x} & =(1-w)\left\{-\beta x\left[N_{c} y\right]-\beta x\left[N_{c} y^{2}\right]\right\}+w\left\{-\beta^{\prime} N_{c} \eta^{0}\right\} \\
\Rightarrow \dot{x} & =-(1-w)\left(N_{c} \beta x\right)\left(y^{2}+y+\frac{\beta^{\prime}}{\beta} \frac{w}{(1-w)}\right)
\end{aligned}
$$

A solução $\eta^{0}=0(x=0)$ é imediata, como esperado. As demais soluções não satisfazem as condições de normalização e devem ser descartadas.

Assumindo o resultado anterior, a equação para $\dot{\eta}^{1}$ torna-se mais simples, como segue:

$$
\begin{aligned}
\dot{\eta^{1}}= & (1-w)\left\{\beta\left[\eta^{2}\left(\eta^{1}\right)^{2}+\eta^{3}\left(\eta^{1}\right)^{2}+\ldots+\eta^{N_{c}}\left(\eta^{1}\right)^{2}\right]-\right. \\
& -\beta\left[\eta^{1}\left(\eta^{2}\right)^{2}+\eta^{1}\left(\eta^{3}\right)^{2}+\ldots+\eta^{1}\left(\eta^{N_{c}}\right)^{2}\right]+ \\
& +w\left\{\beta^{\prime}\left[\eta^{0}+\eta^{2}+\ldots+\eta^{N_{c}}\right]-\beta^{\prime}\left(N_{c}-1\right) \eta^{1}\right\}
\end{aligned}
$$

Novamente, consideramos o comportamento médio dos demais candidatos sobre os eleitores de $\sigma=1$.

$$
\begin{aligned}
& \eta^{1}=z \\
& \eta^{2}=\eta^{3}=\ldots=\eta^{N_{c}}=v
\end{aligned}
$$

A condição de normalização impõe $v=\frac{1-z}{N_{c}-1}$. Portanto:

$$
\begin{aligned}
\dot{z}= & (1-w)\left\{\beta\left(N_{c}-1\right) z^{2} v-\beta\left(N_{c}-1\right) z v^{2}\right\}+ \\
& +w\left\{\beta^{\prime}\left(N_{c}-1\right) v-\beta^{\prime}\left(N_{c}-1\right) z\right\} \\
\Rightarrow \dot{z}= & -(1-w) \beta\left(N_{c}-1\right)(z-v)\left(z v-\frac{\beta^{\prime}}{\beta} \frac{w}{(1-w)}\right)
\end{aligned}
$$

As soluções estacionárias são:

$$
\begin{aligned}
& z=v=\frac{1}{N_{c}} \Rightarrow\left(\eta^{0}=0, \eta^{\sigma}=\frac{1}{N_{c}}\right), \forall \sigma \neq 0 \\
& z=\frac{1}{2} \pm \sqrt{\frac{1}{4}-\left(N_{c}-1\right) \frac{\beta^{\prime}}{\beta} \frac{w}{(1-w)}}
\end{aligned}
$$


Portanto, segue que os pontos fixos para a dinâmica, nesta abordagem simplificada, são:

$$
\begin{aligned}
& \left(\eta^{0}=0, \eta^{\sigma}=\frac{1}{N_{c}}\right), \forall \sigma \neq 0 . \\
& \left(\eta^{0}=0, \eta^{\sigma_{v}}=\eta_{+}^{\sigma},\left\langle\eta^{\sigma}\right\rangle=\eta_{-}^{\sigma}\right), \text { onde }\left\langle\eta^{\sigma}\right\rangle \text { é a média sobre } \sigma \neq 0, \sigma_{v} . \\
& \eta_{ \pm}^{\sigma}=\frac{1}{2} \pm \sqrt{\frac{1}{4}-\left(N_{c}-1\right) \frac{\beta^{\prime}}{\beta} \frac{w}{(1-w)}}
\end{aligned}
$$

Conforme esperado, à campo nulo, temos:

$$
\begin{aligned}
& \left(\eta^{0}=0, \eta^{\sigma}=\frac{1}{N_{c}}\right), \forall \sigma \neq 0 \text { (Equiprobabilidade). } \\
& \left(\eta^{\sigma_{v}}=1, \eta^{\sigma}=0\right), \forall \sigma \neq \sigma_{v} \text { (Consenso). }
\end{aligned}
$$

É razoável esperar que apenas a situação de consenso seja estável à campo nulo, enquanto que a situação de equiprobabilidade pode tornarse estável a partir de uma determinada intensidade de ruído.

No capítulo seguinte, pretendemos tornar os argumentos desta seção mais rigorosos. Utilizaremos o critério da derivada para determinar a estabilidade dos pontos fixos e calcularemos o fator geométrico $\frac{\beta^{\prime}}{\beta}$ de forma explícita. 


\section{Capítulo 3}

\section{Aproximação de Campo Médio}

Neste capítulo iremos discutir, através de uma aproximação de campo médio, as configurações estacionárias do Modelo Sznajd Complexo, explorando suas propriedades e interpretações físicas [6, 36]. A aplicação de um campo externo à dinâmica do modelo (ruído) será considerada, num segundo momento, e interpretada fisicamente como a atuação de campanhas publicitárias na opinião do eleitorado.

\subsection{Aproximação de Campo Médio na Ausência de Ruído}

\subsubsection{Construindo a Equação Mestra}

Definiremos $\eta^{\sigma}(i, t)$ como a probabilidade de que o eleitor i tenha escolhido, no instante t, o candidato $\sigma$. É imediato, pela condição de normalização, que $\sum_{\sigma=0}^{N_{c}} \eta^{\sigma}(i, t)=\eta^{0}(i, t)+\sum_{\sigma=1}^{N_{c}} \eta^{\sigma}(i, t)=1$; onde 
$\eta^{0}(i, t)$ representa a probabilidade de que o eleitor i não tenha escolhido candidato algum no instante t.

Em nossa construção, fixaremos o sítio $i$ considerado acima e através das interações que este sítio pode realizar com seus primeiros vizinhos (que representaremos por $j$ ) ou com seus segundos vizinhos (que representaremos por $j^{\prime}$ ) é que escreveremos cada termo da equação. Por simplicidade e para fixar as idéias, apresentamos a esquematização envolvida na construção da equação para o caso de uma rede quadrada na Figura 3.1.

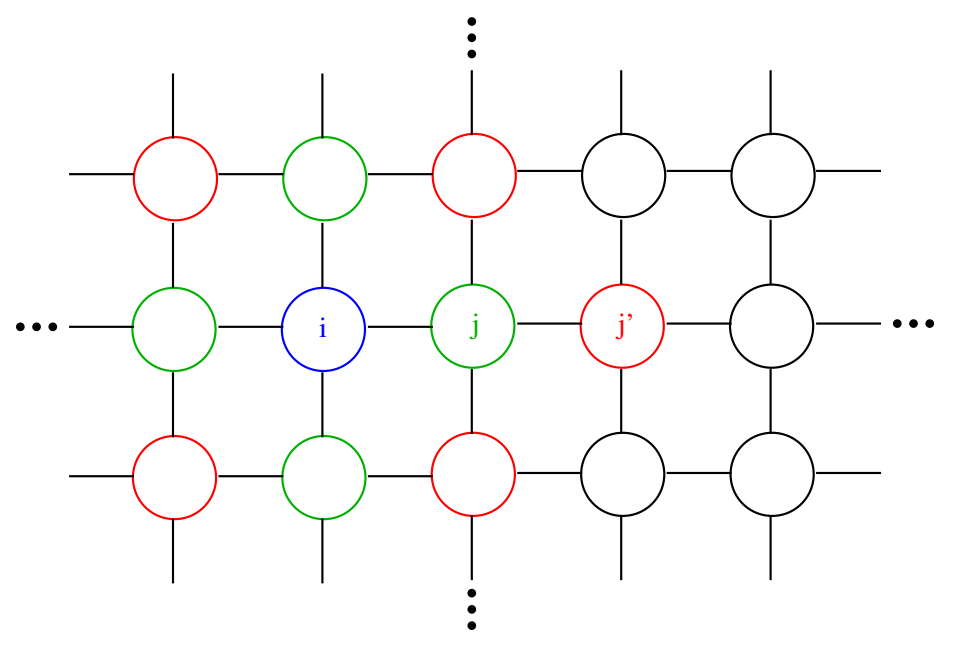

Figura 3.1: O sítio para o qual estamos escrevendo o termo da equação mestra está marcado em azul (e representado pelo índice $i$ ), sua primeira vizinhaça em verde (representados pelo índice $j$ ) e sua segunda vizinhaça em vermelho (representados pelo índice $j^{\prime}$ ). Eventualmente, alguns sítios que pertencem a segunda vizinhaça de $i$ não estão representados na figura.

É conveniente descrevermos a evolução das opiniões abordando separadamente as variações de $\eta^{0}(i, t)$ e $\eta^{\sigma}(i, t)$, devido as distinções impostas pelas regras do modelo aos dois casos. 
A variação de $\eta^{0}(i, t)$ está associada a probabilidade de que, em um tempo de Monte Carlo, o sítio i tenha sido convencido por um de seus vizinhos $j\left(j \in \Gamma_{i}\right)$, passando a votar no candidato $\sigma_{j}{ }^{1}$. Esta variação pode ocorrer de três formas distintas que designaremos por:

1. Confronto Direto de Opiniões: dada a escolha do sítio $j$ pertencente a vizinhança de $\mathrm{i}$, este pode sortear o sítio i com probabilidade $k_{j}^{-1}$ e convencê-lo com probabilidade $k_{j}^{-1}$ a votar em $\sigma_{j}$ (segundo a regra IIa).

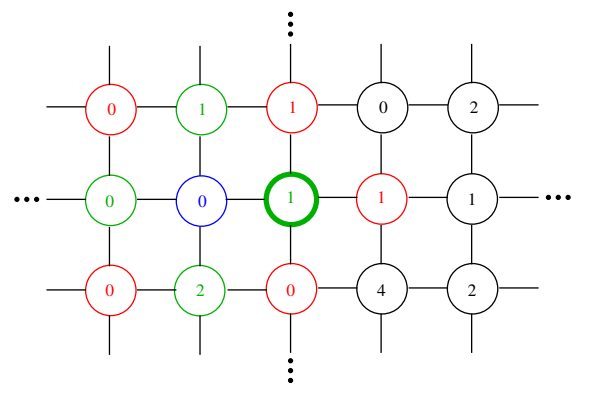

(a)

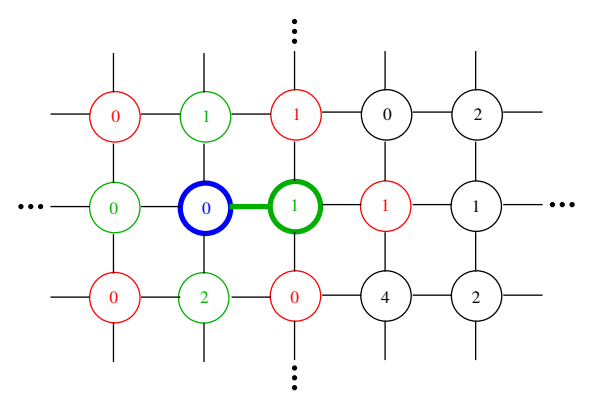

(c)

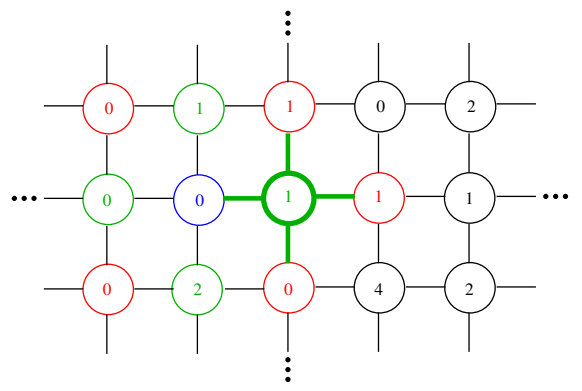

(b)

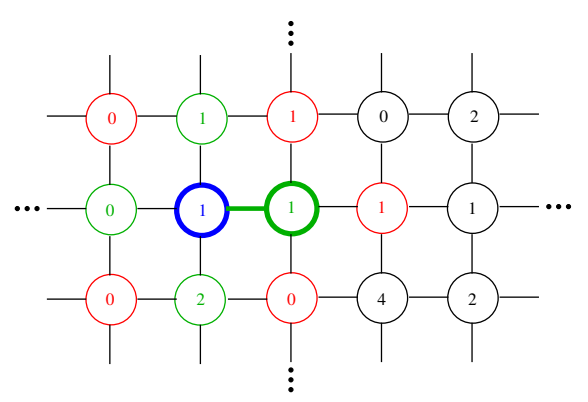

(d)

Figura 3.2: Confronto direto entre $i$ e um de seus vizinhos $j$. (a) Primeiramente, um sítio $j \in \Gamma_{i}$ é escolhido. (b) Em seguida, o sítio sorteado assedia seus vizinhos. (c) $\mathrm{O}$ sítio $j$ escolhe o sítio $i$ fixado. (d) Finalmente, $j$ consegue convencer o sítio fixado a concordar consigo.

\footnotetext{
${ }^{1}$ Forma compactada da notação $\sigma(j, t)$.
} 
2. Concordância entre Eleitores Vizinhos de Primeira Espécie: dada a escolha do sítio $j$ pertencente a vizinhança de i, um dos $k_{j}^{\sigma_{j}}$ vizinhos com mesma opinião pode ser sorteado com probabilidade $k_{j}^{\sigma_{j}} / k_{j}$, possibilitando que $\mathrm{j}$ convença i com probabilidade $k_{j}^{-1}$ a votar em $\sigma_{j}$ (segundo a regra IIb).

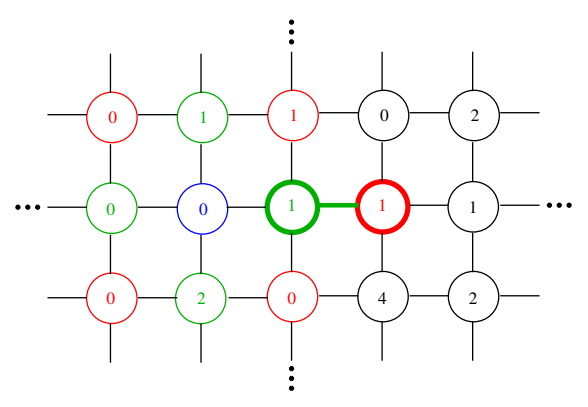

(a)

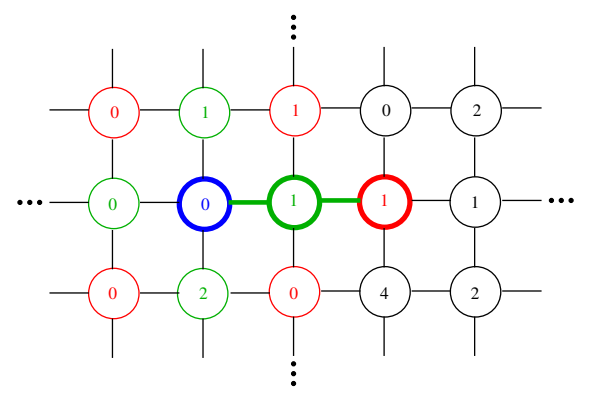

(c)

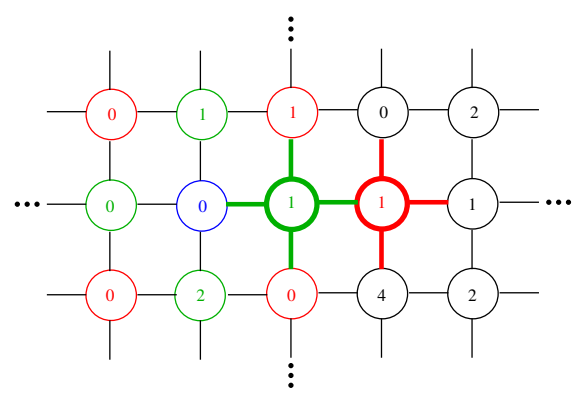

(b)

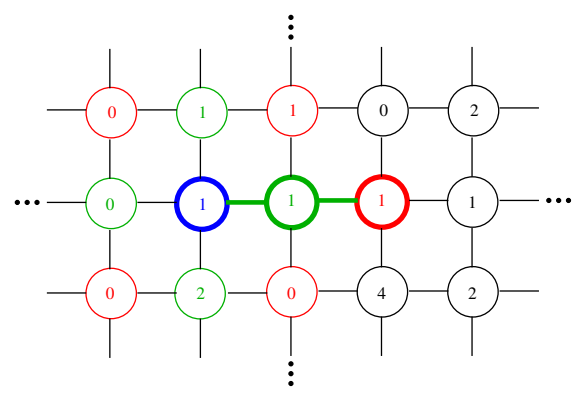

(d)

Figura 3.3: Convencimento através da interação de vizinhos de primeira espécie. (a) Um sítio $j \in \Gamma_{i}$ é sorteado e, por sua vez, escolhe um de seus vizinhos $j^{\prime} \in \gamma_{i}$ de mesma opinião. (b) Ambos os sítios assediam suas respectivas vizinhanças. (c) O sítio $j$ escolhe o sítio $i$ fixado. (d) $j$ consegue convencer o sítio fixado a concordar com a opinião do par.

3. Concordância entre Eleitores Vizinhos de Segunda Espécie: dada a escolha do sítio $j^{\prime}$ pertencente a vizinhança segunda de $i\left(j^{\prime} \in\right.$ 
$\left.\gamma_{i}\right)^{2}$, um dos $\tilde{k}_{j^{\prime}}^{\sigma_{j^{\prime}}}$ vizinhos simultâneos de j' e de i com mesma opinião pode ser sorteado com probabilidade $\tilde{k}_{j^{\prime}}^{\sigma_{j^{\prime}}} / k_{j^{\prime}}$, possibilitando que este vizinho comum $\mathrm{j}$ convença $\mathrm{i}$ com probabilidade $k_{j}^{-1}$ a votar em $\sigma_{j^{\prime}}$ (segundo a regra IIb).

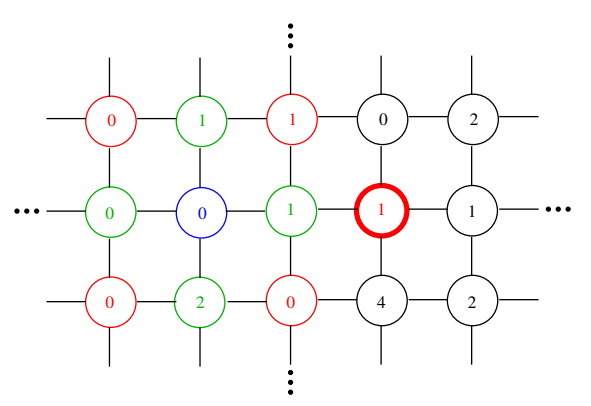

(a)

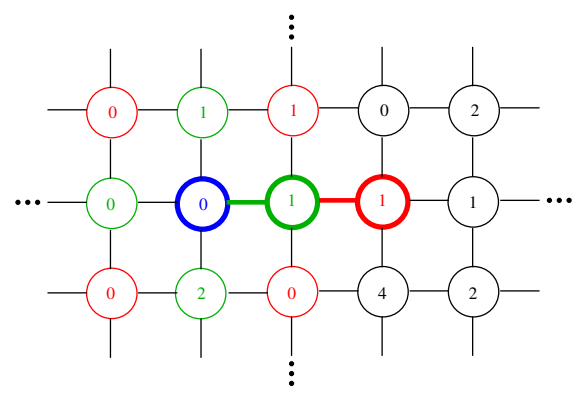

(c)

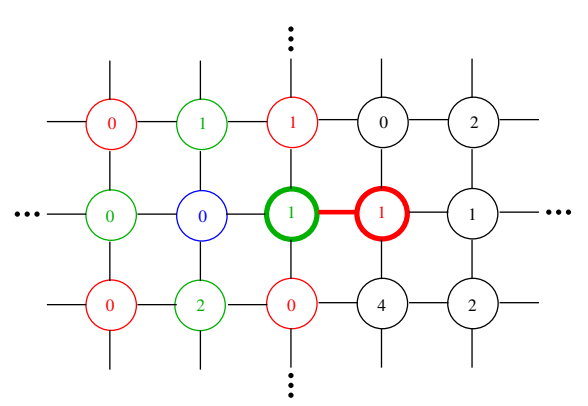

(b)

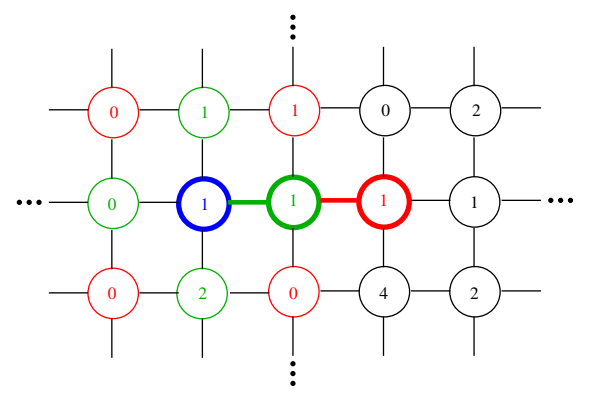

(d)

Figura 3.4: Convencimento através da interação de vizinhos de segunda espécie. (a) Primeiramente, o sítio $j^{\prime} \in \gamma_{i}$ é sorteado. (b) O sítio $j^{\prime}$, após assediar sua vizinhança, escolhe um de seus vizinhos $j \in \Gamma_{i}$. (c) $j$ escolhe o sítio $i$ fixado. (d) $j$ consegue convencer o sítio fixado a concordar com a opinião do par.

$$
{ }^{2} \gamma_{i}=\bigcup_{j} \Gamma_{j}-\left(\{i\} \cup \Gamma_{i}\right) ; j \in \Gamma_{i}
$$




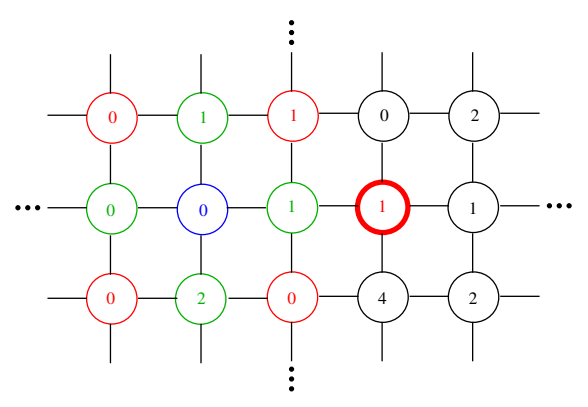

(a)

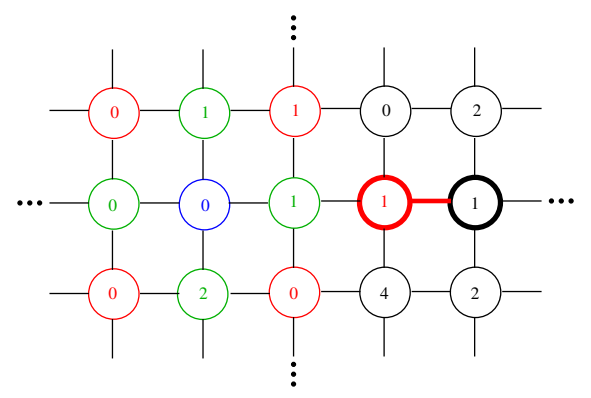

(c)

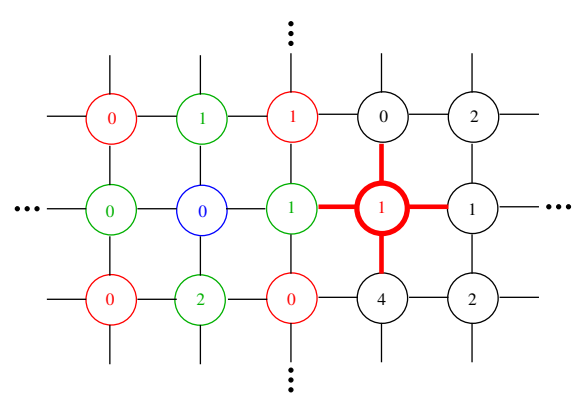

(b)

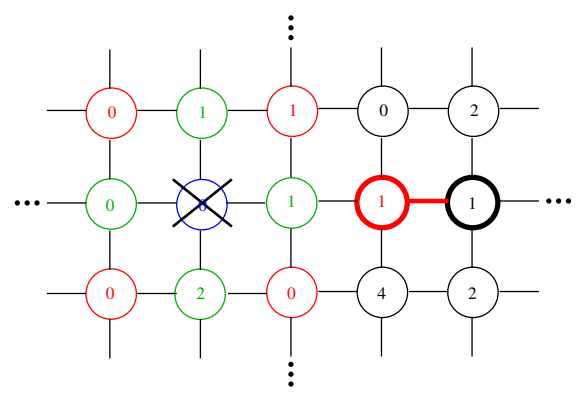

(d)

Figura 3.5: Principal diferença entre as concordâncias de primeira e segunda espécie. (a) Primeiramente, um sítio $j^{\prime} \in \gamma_{i}$ é escolhido. (b) $\mathrm{O}$ sítio $j^{\prime}$ assedia seus vizinhos. (c) $j^{\prime}$ escolhe um de seus vizinhos que pertence a terceira vizinhança do sítio $i$ fixado. (d) Neste caso, o par concordante não é capaz de interagir com o sítio $i$ que está 'fora de seu alcance'. 
Portanto:

$$
\begin{aligned}
\Delta \eta^{0}(i, t)= & \eta^{0}(i, t+1)-\eta^{0}(i, t)= \\
= & -\sum_{j \in \Gamma_{i}} \frac{1}{k_{j}} \frac{1}{k_{j}} P\left(\sigma_{i}=0, \sigma_{j} \neq 0\right)- \\
& -\sum_{j \in \Gamma_{i}} \frac{k_{j}^{\sigma_{j}}}{k_{j}} \frac{1}{k_{j}} P\left(\sigma_{i}=0, \sigma_{j}=\sigma_{j^{\prime}} \neq 0\right)- \\
& -\sum_{\mathbf{j}^{\prime} \in \gamma_{\mathbf{i}}} \frac{\tilde{\mathbf{k}}_{\mathbf{j}^{\prime}}^{\sigma_{\mathbf{j}^{\prime}}}}{\mathbf{k}_{\mathbf{j}^{\prime}}} \frac{\mathbf{1}}{\mathbf{k}_{\mathbf{j}}} \mathbf{P}\left(\sigma_{\mathbf{i}}=\mathbf{0}, \sigma_{\mathbf{j}}=\sigma_{\mathbf{j}^{\prime}} \neq \mathbf{0}\right)
\end{aligned}
$$

Destacamos a terceira somatória da equação por representar o termo acrescentado em relação a abordagem anterior de Vannucchi e Prado.

Em qualquer uma das possibilidades, há sempre uma tendência à redução no número de eleitores indecisos $\left(\eta^{0}(i, t+1) \leq \eta^{0}(i, t)\right)$, determinando assim uma variação negativa na probabilidade de sua ocorrência $\left(\Delta \eta^{0}(i, t) \leq 0\right)^{3}$. Devido as regras do modelo, é descartada a possibilidade de um eleitor que, no instante t, votava em determinado candidato $\sigma$, retome um estado de indecisão, impedindo parcelas com sinal positivo na equação.

A variação de $\eta^{\sigma}(i, t)$ é mais delicada, pois, além de considerarmos possibilidades semelhantes ao caso de $\eta^{0}(i, t)$ (confronto direto, concordâncias de primeira e segunda espécie) é necessário avaliar também, como as intenções de voto no candidato $\sigma$ podem aumentar ou diminuir quando o eleitor i interage com sua vizinhança. Desta forma, esperamos parcelas com sinal positivo e negativo na equação mestra.

Vejamos como se dá a variação de $\eta^{\sigma}(i, t)$.

1. Confronto Direto de Opiniões: neste caso, temos apenas possibilidades de aumento nas intenções de votos ${ }^{4}$.

\footnotetext{
${ }^{3} \mathrm{Na}$ pior das hipóteses, a probabilidade de encontrarmos um eleitor indeciso se mantém inalterada em um TMC, caso o eleitor i não seja convencido por $j$.

${ }^{4}$ Pela regras do modelo, se $\sigma_{i}=\sigma \neq 0$, então, num confronto direto entre qualquer um
} 
1.1 Aumento nas Intenções de Voto: caso i esteja indeciso e escolhendo-se o sítio $j \in \Gamma_{i}$ com $\sigma_{j}=\sigma$, o sítio i pode ser sorteado com probabilidade $k_{j}^{-1}$ e convencido por $\mathrm{j}$ com probabilidade $k_{j}^{-1}$ a votar em $\sigma_{j}=\sigma$ (segundo a regra IIa).

2. Concordância entre Eleitores Vizinhos de Primeira Espécie:

2.1. Aumento nas Intenções de Voto: dada a escolha do sítio $j \in \Gamma_{i}$ com opinião $\sigma_{j}=\sigma$, um dos $k_{j}^{\sigma_{j}}$ vizinhos com mesma opinião pode ser sorteado com probabilidade $k_{j}^{\sigma_{j}} / k_{j}$, possibilitando que $\mathrm{j}$ convença i com probabilidade $k_{j}^{-1}$ a votar em $\sigma$ (segundo a regra IIb).

2.2. Diminuição nas Intenções de Voto: caso i tenha escolhido o candidato $\sigma$ e escolhendo-se o sítio $j \in \Gamma_{i}$ que vota em $\sigma^{\prime} \neq \sigma$, um dos $k_{j}^{\sigma_{j}}$ vizinhos de $\mathrm{j}$ com mesma opinião pode ser sorteado com probabilidade $k_{j}^{\sigma_{j}} / k_{j}$, possibilitando que j convença i com probabilidade $k_{j}^{-1}$ a deixar de votar em $\sigma$ e passar a votar em $\sigma^{\prime}$ (segundo a regra $\mathrm{IIb}$ ).

3. Concordância entre Eleitores Vizinhos de Segunda Espécie:

3.1. Aumento nas Intenções de Voto: dada a escolha do sítio $j^{\prime}$ pertencente a vizinhança segunda de i, tal que $\sigma_{j^{\prime}}=\sigma$, um dos $\tilde{k}_{j^{\prime}}^{\sigma_{j^{\prime}}}$ vizinhos simultâneos de j' e de i com mesma opinião pode ser sorteado com probabilidade $\tilde{k}_{j^{\prime}}^{\sigma^{\prime}} / k_{j^{\prime}}$, possibilitando que este vizinho comum, convença i com probabilidade $k_{j}^{-1}$ a votar no candidato $\sigma$ (segundo a regra $\mathrm{IIb}$ ).

3.2. Diminuição nas Intenções de Voto: caso i tenha escolhido o candidato $\sigma$ e escolhendo-se o sítio $j^{\prime}$ pertencente a vizinhança se-

de seus vizinhos, a opinião de i não mudará, impossibilitando diminuição nas intenções de voto para o candidato $\sigma$. 
gunda de i, tal que $\sigma_{j^{\prime}}=\sigma_{j}=\sigma^{\prime} \neq \sigma$, um dos $\tilde{k}_{j^{\prime}}^{\sigma_{j^{\prime}}}$ vizinhos simultâneos de j' e de i com mesma opinião pode ser sorteado com probabilidade $\tilde{k}_{j^{\prime}}^{\sigma_{j^{\prime}}} / k_{j^{\prime}}$, possibilitando que este vizinho comum convença i com probabilidade $k_{j}^{-1}$ a deixar de votar em $\sigma$ e passar a votar em $\sigma^{\prime}$ (segundo a regra IIb).

Portanto:

$$
\begin{aligned}
\Delta \eta^{\sigma}= & \sum_{j \in \Gamma_{i}} \frac{1}{k_{j}} \frac{1}{k_{j}} P\left(\sigma_{i}=0, \sigma_{j}=\sigma\right)+ \\
& +\sum_{j \in \Gamma_{i}} \frac{k_{j}^{\sigma_{j}}}{k_{j}} \frac{1}{k_{j}} P\left(\sigma_{i} \neq \sigma, \sigma_{j}=\sigma_{j^{\prime}}=\sigma\right)- \\
& -\sum_{\substack{\sigma \prime=1 \\
\left(\sigma^{\prime} \neq \sigma\right)}}^{N_{c}} \sum_{j \in \Gamma_{i}} \frac{k_{j}^{\sigma_{j}}}{k_{j}} \frac{1}{k_{j}} P\left(\sigma_{i}=\sigma, \sigma_{j}=\sigma_{j^{\prime}}=\sigma^{\prime}\right)+ \\
& +\sum_{\substack{\mathbf{j}^{\prime} \in \gamma_{\mathbf{i}} \\
\mathbf{k}_{\mathbf{j}^{\prime}}}} \frac{\mathbf{1}}{\mathbf{k}_{\mathbf{j}^{\prime}}} \frac{\mathbf{k}_{\mathbf{j}}}{\mathbf{j}_{\mathbf{j}}} \mathbf{P}\left(\sigma_{\mathbf{i}} \neq \sigma, \sigma_{\mathbf{j}}=\sigma_{\mathbf{j}^{\prime}}=\sigma\right)- \\
& -\sum_{\substack{\sigma^{\prime}=1 \\
\left(\sigma^{\prime} \neq \sigma\right)}}^{\mathbf{N}_{\mathbf{c}}} \sum_{\mathbf{j}^{\prime} \in \gamma_{\mathbf{i}}} \frac{\tilde{\mathbf{k}}_{\mathbf{j}^{\prime}}^{\sigma_{\mathbf{j}^{\prime}}}}{\mathbf{k}_{\mathbf{j}^{\prime}}} \frac{\mathbf{1}}{\mathbf{k}_{\mathbf{j}}} \mathbf{P}\left(\sigma_{\mathbf{i}}=\sigma, \sigma_{\mathbf{j}}=\sigma_{\mathbf{j}^{\prime}}=\sigma^{\prime}\right)
\end{aligned}
$$

Novamente, as somatórias destacadas representam os termos acrescentados em relação a abordagem anterior de Vannucchi e Prado.

Uma abordagem exata das equações (3.1) e (3.2) é muito complicada, tornando conveniente a aproximação de campo médio.

\subsubsection{Hipóteses para a Aproximação de Campo Médio}

Faremos então, a aproximação de campo médio, considerando as seguintes hipóteses:

1. Homogeneidade espacial.

$$
\eta(i, t) \equiv \eta(t)
$$


2. Independência estatística entre a opinião de cada eleitor e a de seus vizinhos.

$$
\begin{aligned}
& P\left(\sigma_{i}=0, \sigma_{j} \neq 0\right)=P\left(\sigma_{i}=0\right) P\left(\sigma_{j} \neq 0\right)=\eta^{0}\left(1-\eta^{0}\right) \\
& \begin{aligned}
P\left(\sigma_{i}=0, \sigma_{j}=\sigma_{j^{\prime}} \neq 0\right) & =P\left(\sigma_{i}=0\right) P\left(\sigma_{j}=\sigma_{j}^{\prime} \neq 0\right) \\
& =\eta^{0}\left(1-\eta^{0}\right)
\end{aligned} \\
& \begin{aligned}
& P\left(\sigma_{i}=0, \sigma_{j}=\sigma\right)=P\left(\sigma_{i}=0\right) P\left(\sigma_{j}=\sigma\right)=\eta^{0} \eta^{\sigma} \\
& P\left(\sigma_{i} \neq \sigma, \sigma_{j}=\sigma_{j^{\prime}}=\sigma\right)=P\left(\sigma_{i} \neq \sigma\right) P\left(\sigma_{j}=\sigma_{j^{\prime}}=\sigma\right) \\
&=\left(1-\eta^{\sigma}\right) \eta^{\sigma}
\end{aligned} \\
& \begin{aligned}
P\left(\sigma_{i}=\sigma, \sigma_{j}=\sigma_{j^{\prime}}=\sigma^{\prime} \neq \sigma\right) & =P\left(\sigma_{i}=\sigma\right) P\left(\sigma_{j}=\sigma_{j^{\prime}} \neq \sigma\right) \\
& =\eta^{\sigma} \eta^{\sigma^{\prime}}
\end{aligned} \\
& \text { Assumiremos ainda que: } \eta^{\sigma^{\prime}}=\frac{1-\eta^{0}-\eta^{\sigma}}{N_{c}-1}
\end{aligned}
$$

3. Substituir variáveis locais por suas respectivas médias sobre todos os vértices da rede.

$$
\begin{aligned}
& k_{i} \rightarrow\langle k\rangle \\
& k_{i}^{\prime} \rightarrow\left\langle k^{\prime}\right\rangle \\
& k_{j} \rightarrow\left\langle k_{v}\right\rangle \\
& k_{j^{\prime}} \rightarrow\left\langle k_{v}^{\prime}\right\rangle \\
& k_{j}^{\sigma_{j}} \rightarrow\left\langle k_{v}\right\rangle \eta^{\sigma_{j}} \\
& \tilde{k}_{j^{\prime}}^{\sigma_{j^{\prime}}} \rightarrow\langle l\rangle\left\langle k_{v}^{\prime}\right\rangle \eta^{\sigma_{j^{\prime}}} .
\end{aligned}
$$

Perceba que, apesar de termos descartado correlações espaciais com o estabelecimentos destas hipóteses, o tipo de rede que consideramos para a realização da dinâmica é levado em conta através dos parâmetros médios que a caracterizam, a saber, o grau médio dos vértices $\langle k\rangle$, o grau médio dos vértices da segunda vizinhança $\left\langle k_{v}\right\rangle$, o número médio de segundos vizinhos $\left\langle k^{\prime}\right\rangle$ e o fator de acoplamento entre os primeiros e segundos vizinhos $\langle l\rangle$. Além disso, os parâmetros $\left\langle k_{v}\right\rangle$ e $\langle l\rangle$ devem 
ser definidos como segue: para cada vértice da rede obtem-se o valor médio do parâmetro em questão, em seguida, toma-se a média destes valores para todos os vértices.

O fator de acoplamento $\langle l\rangle$ reflete o fato de que apenas a fração de vértices comuns entre os elementos da primeira e da segunda vizinhança do sítio considerado que é que deve ser levada em conta na estimativa da taxa de transição média em interações de segunda espécie. Esperamos que o valor típico de $\langle l\rangle$ seja pequeno quando comparado aos demais parâmetros médios da rede, conferindo um caráter desprezível para transições de segunda espécie, em concordância com a abordagem de Vannucchi em sua dissertação.

Portanto, podemos reescrever a equação mestra numa versão de campo médio como segue:

$$
\begin{gathered}
\Delta \eta^{0}=-\frac{1}{\left\langle k_{v}\right\rangle} \eta^{0}\left(1-\eta^{0}\right)\left[\frac{\langle k\rangle}{\left\langle k_{v}\right\rangle}+\left(\langle k\rangle+l\left\langle k^{\prime}\right\rangle\right)\left(1-\eta^{0}\right)\right] \\
\Delta \eta^{\sigma}=\frac{\eta^{\sigma}}{\left\langle k_{v}\right\rangle}\left[\frac{\langle k\rangle}{\left\langle k_{v}\right\rangle} \eta^{0}+\langle k\rangle\left(1-\eta^{\sigma}\right) \eta^{\sigma}-\langle k\rangle \frac{\left(1-\eta^{0}-\eta^{\sigma}\right)^{2}}{N_{c}-1}+\right. \\
\left.+\langle l\rangle\left\langle k^{\prime}\right\rangle\left(1-\eta^{\sigma}\right) \eta^{\sigma}-\langle l\rangle\left\langle k^{\prime}\right\rangle \frac{\left(1-\eta^{0}-\eta^{\sigma}\right)^{2}}{N_{c}-1}\right]
\end{gathered}
$$

\subsubsection{Determinando os Estados Estacionários}

Estamos interessados em encontrar as configurações estacionárias do modelo, isto é, as configurações em que $\Delta \eta=0$.

Impondo a condição de estacionaridade para $\Delta \eta^{0}$, obtemos:

$$
\begin{gathered}
\Delta \eta^{0}=-\frac{1}{\left\langle k_{v}\right\rangle} \eta^{0}\left(1-\eta^{0}\right)\left[\frac{\langle k\rangle}{\left\langle k_{v}\right\rangle}+\left(\langle k\rangle+\langle l\rangle\left\langle k^{\prime}\right\rangle\right)\left(1-\eta^{0}\right)\right]=0 \\
\eta^{0}=1 \text { (contraria o processo de semeadura) } \\
\eta^{0}=0 \\
\eta^{0}=1+\frac{\langle k\rangle}{\left\langle k_{v}\right\rangle\left(\langle k\rangle+\langle l\rangle\left\langle k^{\prime}\right\rangle\right)} \text { (contraria a normalização) }
\end{gathered}
$$


Portanto, a única solução aceitável fisicamente é $\eta^{0}=0$.

Este resultado era esperado, pois, a configuração em que nenhum eleitor está indeciso é claramente estacionária (nenhum eleitor pode retomar o estado de indecisão), além disso, é uma configuração estável, dado que, surgindo um pequeno número de indecisos, ele tende a se extinguir.

Agora, impondo a condição de estacionariedade para $\Delta \eta^{\sigma}$, obtemos:

$$
\begin{aligned}
\Rightarrow \Delta \eta^{\sigma}=\frac{\eta^{\sigma}}{\left\langle k_{v}\right\rangle} & {\left[\frac{\langle k\rangle}{\left\langle k_{v}\right\rangle} \eta^{0}+\langle k\rangle\left(1-\eta^{\sigma}\right) \eta^{\sigma}-\langle k\rangle \frac{\left(1-\eta^{0}-\eta^{\sigma}\right)^{2}}{N_{c}-1}+\right.} \\
& \left.+\langle l\rangle\left\langle k^{\prime}\right\rangle\left(1-\eta^{\sigma}\right) \eta^{\sigma}-\langle l\rangle\left\langle k^{\prime}\right\rangle \frac{\left(1-\eta^{0}-\eta^{\sigma}\right)^{2}}{N_{c}-1}\right]=0
\end{aligned}
$$

Mas, $\eta^{0}=0$. Desta forma:

$$
\begin{gathered}
\Delta \eta^{\sigma}=\frac{\eta^{\sigma}}{\left\langle k_{v}\right\rangle}\left[\langle k\rangle\left(1-\eta^{\sigma}\right) \eta^{\sigma}-\langle k\rangle \frac{\left(1-\eta^{\sigma}\right)^{2}}{N_{c}-1}+\right. \\
\left.+\langle l\rangle\left\langle k^{\prime}\right\rangle\left(1-\eta^{\sigma}\right) \eta^{\sigma}-\langle l\rangle\left\langle k^{\prime}\right\rangle \frac{\left(1-\eta^{\sigma}\right)^{2}}{N_{c}-1}\right]=0 \\
\Rightarrow \Delta \eta^{\sigma}=\frac{\eta^{\sigma}}{\left\langle k_{v}\right\rangle}\left(1-\eta^{\sigma}\right)\left(\langle k\rangle+\langle l\rangle\left\langle k^{\prime}\right\rangle\right) \frac{\left(\eta^{\sigma}-\frac{1}{N_{c}}\right)}{N_{c}-1}=0 \\
\Rightarrow\left\{\begin{array}{c}
\eta^{\sigma}=0 \\
\eta^{\sigma}=1 \\
\eta^{\sigma}=\frac{1}{N_{c}}
\end{array}\right.
\end{gathered}
$$

Na realidade, dado que existem vários candidatos, as soluções correspondem a duas configurações distintas, a saber, $\left(\eta^{\sigma}=0, \eta^{\sigma_{v}}=1\right), \forall \sigma \neq$ $\sigma_{v}$, onde $\sigma_{v}$ representa o candidato vencendor (estado de consenso) ou $\left(\eta^{0}=0, \eta^{\sigma}=\frac{1}{N_{c}}\right), \forall \sigma \neq 0$ (estado de equiprobabilidade entre os candidatos). A configuração de equiprobabilidade entre os candidatos pode ser interpretada fisicamente como uma situação de empate técnico entre todos os candidatos. 
A estabilidade destas configurações pode ser obtida pela linearização de $\Delta \eta$ na vizinhança de seus valores estacionários. Assim:

$$
\begin{gathered}
\left.\frac{\partial}{\partial \eta^{0}}\left(\Delta \eta^{0}\right)\right|_{\eta^{0}=0}=-\frac{\langle k\rangle+\langle k\rangle\left\langle k_{v}\right\rangle+\langle l\rangle\left\langle k^{\prime}\right\rangle\left\langle k_{v}\right\rangle}{\left\langle k_{v}\right\rangle^{2}} \Rightarrow \eta^{0}=0 \text { é estável } \\
\left.\frac{\partial}{\partial \eta^{\sigma}}\left(\Delta \eta^{\sigma}\right)\right|_{\eta^{\sigma}=0}=-\frac{\left(\langle k\rangle+\langle l\rangle\left\langle k^{\prime}\right\rangle\right)}{\left\langle k_{v}\right\rangle\left(N_{c}-1\right)}<0 \Rightarrow \eta^{\sigma}=0 \text { é estável } \\
\left.\frac{\partial}{\partial \eta^{\sigma}}\left(\Delta \eta^{\sigma}\right)\right|_{\eta^{\sigma}=1}=-\frac{\left(\langle k\rangle+\langle l\rangle\left\langle k^{\prime}\right\rangle\right)}{\left\langle k_{v}\right\rangle}<0 \Rightarrow \eta^{\sigma}=1 \text { é estável } \\
\left.\frac{\partial}{\partial \eta^{\sigma}}\left(\Delta \eta^{\sigma}\right)\right|_{\eta^{\sigma}=\frac{1}{N_{c}}}=\frac{\left(\langle k\rangle+\langle l\rangle\left\langle k^{\prime}\right\rangle\right)}{\left\langle k_{v}\right\rangle N_{c}}>0 \Rightarrow \eta^{\sigma}=\frac{1}{N_{c}} \text { é instável }
\end{gathered}
$$

Assim, a configuração de consenso é estável, enquanto que a configuração de empate técnico entre os candidatos é instável.

Como uma situação instável depende de condições iniciais bastante peculiares e muito improváveis do ponto de vista prático, podemos concluir que o Modelo Sznajd Complexo sempre evolui para um estado de consenso no regime estacionário, assim como o Modelo Sznajd original.

\subsection{Aproximação de Campo Médio com Ruído}

\subsubsection{Construindo a Equação Mestra}

A equação mestra no caso com ruído é analoga ao caso sem ruído, como segue. Por simplicidade, faremos a construção considerando diretamente uma aproximação de campo médio.

Os termos que correspondem a aproximação de campo médio sem ruído são multiplicados por um fator $(1-w)$, que representa a probabilidade dos vértices em seguir a dinâmica convencional, os outros termos a serem acrescentados representarão os sítios que optaram pela escolha aleatória de um candidato (eleitores convecidos por uma campanha publicitária) e, por isso, são multiplicados por $w$. 
Para a equação que descreve a probabilidade de eleitores indecisos $\left(\Delta \eta^{0}\right)$, é imediato que devemos acrescentar o termo $-w \eta^{0}$, pois um sítio indeciso que é afetado pelo ruído sempre deixa o estado de indecisão e passa a votar em qualquer outro candidato com probabilidade 1 .

Para a equação que descreve a probabilidade de votos referentes a um determinado candidato $\sigma\left(\Delta \eta^{\sigma}\right)$, devemos considerar duas parcelas, uma referente ao ruído contribuir com um aumento nas intenções de votos e outra referente ao ruído contribuir com uma diminuição. Uma contribuição que aumenta as intenções de votos corresponde ao acréscimo do termo $+w\left(1-\eta^{\sigma}\right)\left(\frac{1}{N_{c}}\right)$ por representar a probabilidade do evento composto que o favorece: o primeiro fator é a probabilidade do sítio considerado ser afetado pelo ruído, o segundo fator é a probabilidade do mesmo não votar em sigma, e, o terceiro fator, é a probabilidade dele passar a votar em $\sigma$ após ser alvo da campanha publicitária. Semelhantemente, a contribuição que diminui as intenções de votos corresponde ao acréscimo do termo $-w \eta^{\sigma}\left(1-\frac{1}{N_{c}}\right)$ por representar a probabilidade do evento composto que o desfavorece: o primeiro fator é a probabilidade do sítio considerado ser afetado pelo ruído, o segundo fator é a probabilidade do mesmo votar em sigma, e, o terceiro fator, é a probabilidade dele passar a votar em outro candidato após ser alvo da campanha publicitária.

Portanto, as equações para o MSC com ruído são:

$$
\begin{aligned}
\Delta \eta^{0}= & -(1-w) \frac{\eta^{0}}{\left\langle k_{v}\right\rangle}\left(1-\eta^{0}\right)\left[\frac{\langle k\rangle}{\left\langle k_{v}\right\rangle}+\left(\langle k\rangle+\langle l\rangle\left\langle k^{\prime}\right\rangle\right)\left(1-\eta^{0}\right)\right]- \\
& -w \eta^{0}
\end{aligned}
$$




$$
\begin{aligned}
\Delta \eta^{\sigma}=(1-w) \frac{\eta^{\sigma}}{\left\langle k_{v}\right\rangle} & {\left[\frac{\langle k\rangle}{\left\langle k_{v}\right\rangle} \eta^{0}+\langle k\rangle\left(1-\eta^{\sigma}\right) \eta^{\sigma}-\right.} \\
& -\langle k\rangle \frac{\left(1-\eta^{0}-\eta^{\sigma}\right)^{2}}{\left(N_{c}-1\right)}+\langle l\rangle\left\langle k^{\prime}\right\rangle\left(1-\eta^{\sigma}\right) \eta^{\sigma}- \\
& \left.-\langle l\rangle\left\langle k^{\prime}\right\rangle \frac{\left(1-\eta^{0}-\eta^{\sigma}\right)^{2}}{\left(N_{c}-1\right)}\right]+ \\
& +w\left[\left(1-\eta^{\sigma}\right) \frac{1}{N_{c}}-\eta^{\sigma}\left(1-\frac{1}{N_{c}}\right)\right]
\end{aligned}
$$

\subsubsection{Determinando os Estados Estacionários}

Vamos determinar as configurações estácionárias do modelo com ruído:

Impondo a condição de estacionariedade para $\Delta \eta^{0}$, obtemos:

$$
\begin{gathered}
(1-w) \frac{\eta^{0}}{\left\langle k_{v}\right\rangle}\left(1-\eta^{0}\right)\left[\frac{\langle k\rangle}{\left\langle k_{v}\right\rangle}+\left(\langle k\rangle+\langle l\rangle\left\langle k^{\prime}\right\rangle\right)\left(1-\eta^{0}\right)\right]+w \eta^{0}=0 \\
\therefore \quad \\
\eta^{0}=0 \\
\text { ou } \\
(1-w) \frac{1}{\left\langle k_{v}\right\rangle}\left(1-\eta^{0}\right)\left[\frac{\langle k\rangle}{\left\langle k_{v}\right\rangle}+\left(\langle k\rangle+\langle l\rangle\left\langle k^{\prime}\right\rangle\right)\left(1-\eta^{0}\right)\right]+w=0
\end{gathered}
$$

Segue ainda que:

$$
\begin{aligned}
\eta^{0}= & +\frac{\langle k\rangle}{2\left\langle k_{v}\right\rangle\left(\langle k\rangle+\langle l\rangle\left\langle k^{\prime}\right\rangle\right)} \pm \\
& \pm \sqrt{\left[\frac{\langle k\rangle}{2\left\langle k_{v}\right\rangle\left(\langle k\rangle+\langle l\rangle\left\langle k^{\prime}\right\rangle\right)}\right]^{2}-\frac{w\left\langle k_{v}\right\rangle}{(1-w)\left(\langle k\rangle+\langle l\rangle\left\langle k^{\prime}\right\rangle\right)}}
\end{aligned}
$$

As soluções não nulas contrariam a condição de normalização e, portanto, a única solução aceitável fisicamente é $\eta^{0}=0$. 
Agora, impondo a condição de estacionariedade para $\Delta \eta^{\sigma}$, com a condição adicional $\eta^{0}=0$, obtemos:

$$
\begin{gathered}
\left(\eta^{\sigma}-\frac{1}{N_{c}}\right)\left[(1-w) \frac{N_{c}}{\left(N_{c}-1\right)} \frac{\left(\langle k\rangle+\langle l\rangle\left\langle k^{\prime}\right\rangle\right)}{\left\langle k_{v}\right\rangle} \eta^{\sigma}\left(1-\eta^{\sigma}\right)-w\right]=0 \\
\therefore \quad \\
\eta^{\sigma}=\frac{1}{N_{c}} \\
\quad \mathrm{ou} \\
(1-w) \frac{N_{c}}{N_{c}-1} \frac{\left(\langle k\rangle+\langle l\rangle\left\langle k^{\prime}\right\rangle\right)}{\left\langle k_{v}\right\rangle} \eta^{\sigma}\left(1-\eta^{\sigma}\right)-w=0
\end{gathered}
$$

Implicando ainda em:

$$
\eta^{\sigma}=\frac{1}{2} \pm \sqrt{\frac{1}{4}-\frac{w}{(1-w)} \frac{\left\langle k_{v}\right\rangle}{\left(\langle k\rangle+\langle l\rangle\left\langle k^{\prime}\right\rangle\right)} \frac{\left(N_{c}-1\right)}{N_{c}}}
$$

Portanto, as soluções para $\Delta \eta^{\sigma}=0$ são:

$$
\begin{aligned}
\eta_{0}^{\sigma} & =\frac{1}{N_{c}} \\
\eta_{+}^{\sigma} & =\frac{1}{2}+\sqrt{\frac{1}{4}-\frac{w}{(1-w)} \frac{\left\langle k_{v}\right\rangle}{\left(\langle k\rangle+\langle l\rangle\left\langle k^{\prime}\right\rangle\right)} \frac{\left(N_{c}-1\right)}{N_{c}}} \\
\eta_{-}^{\sigma} & =\frac{1}{2}-\sqrt{\frac{1}{4}-\frac{w}{(1-w)} \frac{\left\langle k_{v}\right\rangle}{\left(\langle k\rangle+\langle l\rangle\left\langle k^{\prime}\right\rangle\right)} \frac{\left(N_{c}-1\right)}{N_{c}}}
\end{aligned}
$$

Analisando a condição de existência para as soluções $\eta_{+}^{\sigma}$ e $\eta_{-}^{\sigma}$ temos:

$$
\begin{aligned}
& \frac{1}{4}-\frac{w}{(1-w)} \frac{\left\langle k_{v}\right\rangle}{\left(\langle k\rangle+\langle l\rangle\left\langle k^{\prime}\right\rangle\right)} \frac{\left(N_{c}-1\right)}{N_{c}} \geq 0 \\
& \Rightarrow w \leq\left[1+4 \frac{\left\langle k_{v}\right\rangle}{\left(\langle k\rangle+\langle l\rangle\left\langle k^{\prime}\right\rangle\right)} \frac{\left(N_{c}-1\right)}{N_{c}}\right]^{-1}
\end{aligned}
$$

É conveniente definir:

$$
w_{t} \equiv\left[1+4 \frac{\left\langle k_{v}\right\rangle}{\left(\langle k\rangle+\langle l\rangle\left\langle k^{\prime}\right\rangle\right)} \frac{\left(N_{c}-1\right)}{N_{c}}\right]^{-1}<1
$$




$$
\Rightarrow w \leq w_{t}
$$

Em seguida, vamos analisar a estabilidade das soluções:

$$
\begin{aligned}
\left.\frac{\partial}{\partial \eta^{0}}\left(\Delta \eta^{0}\right)\right|_{\eta^{0}=0} & =-\frac{(1-w)}{\left\langle k_{v}\right\rangle}\left(1-\eta^{0}\right)\left[\frac{\langle k\rangle}{\left\langle k_{v}\right\rangle}+\right. \\
& \left.+\left(\langle k\rangle+\langle l\rangle\left\langle k^{\prime}\right\rangle\right)\left(1-\eta^{0}\right)\right]-w \\
& =-\left\{\frac { ( 1 - w ) } { \langle k _ { v } \rangle } ( 1 - \eta ^ { 0 } ) \left[\frac{\langle k\rangle}{\left\langle k_{v}\right\rangle}+\right.\right. \\
& \left.\left.+\left(\langle k\rangle+\langle l\rangle\left\langle k^{\prime}\right\rangle\right)\left(1-\eta^{0}\right)\right]+w\right\} \\
& <0 \\
\Rightarrow & \eta^{0}=0 \quad \text { é estável }
\end{aligned}
$$

Temos também:

$$
\left.\Rightarrow \frac{\partial}{\partial \eta^{\sigma}}\left(\Delta \eta^{\sigma}\right)\right|_{\eta^{\sigma}=\frac{1}{N_{c}}=\eta_{0}^{\sigma}}=(1-w) \frac{\left(\langle k\rangle+\langle l\rangle\left\langle k^{\prime}\right\rangle\right)}{\left\langle k_{v}\right\rangle N_{c}}-w
$$

Para que $\eta_{0}^{\sigma}$ seja estável é necessário que:

$$
\begin{aligned}
& (1-w) \frac{\left(\langle k\rangle+\langle l\rangle\left\langle k^{\prime}\right\rangle\right)}{\left\langle k_{v}\right\rangle N_{c}}-w<0 \\
& \Rightarrow w>\left[1+\frac{\left\langle k_{v}\right\rangle N_{c}}{\left(\langle k\rangle+\langle l\rangle\left\langle k^{\prime}\right\rangle\right)}\right]^{-1}
\end{aligned}
$$

É conveniente definir:

$$
\begin{gathered}
\tilde{w} \equiv\left[1+\frac{\left\langle k_{v}\right\rangle N_{c}}{\left(\langle k\rangle+\langle l\rangle\left\langle k^{\prime}\right\rangle\right)}\right]^{-1}<1 \\
\Rightarrow w>\tilde{w}
\end{gathered}
$$


Agora, analisando a estabilidade das soluções $\eta_{+}^{\sigma}$ e $\eta_{-}^{\sigma}$ :

$$
\begin{aligned}
& \left.\frac{\partial}{\partial \eta^{\sigma}}\left(\Delta \eta^{\sigma}\right)\right|_{\eta^{\sigma}=\eta_{+}^{\sigma}}=(1-w) \frac{\left(\langle k\rangle+\langle l\rangle\left\langle k^{\prime}\right\rangle\right)}{\left\langle k_{v}\right\rangle} \times \\
& \times\left(\frac{N_{c}}{N_{c}-1}\right)\left\{-\frac{1}{2}-\left(\frac{N_{c}-2}{N_{c}}\right) \times\right. \\
& \left.\times \sqrt{\frac{1}{4}-\frac{w}{(1-w)} \frac{\left\langle k_{v}\right\rangle}{\left(\langle k\rangle+\langle l\rangle\left\langle k^{\prime}\right\rangle\right)} \frac{\left(N_{c}-1\right)}{N_{c}}}\right\}+ \\
& +2 w \\
& \left.\frac{\partial}{\partial \eta^{\sigma}}\left(\Delta \eta^{\sigma}\right)\right|_{\eta^{\sigma}=\eta_{-}^{\sigma}}=(1-w) \frac{\left(\langle k\rangle+\langle l\rangle\left\langle k^{\prime}\right\rangle\right)}{\left\langle k_{v}\right\rangle} \times \\
& \times\left(\frac{N_{c}}{N_{c}-1}\right) \times\left\{-\frac{1}{2}+\left(\frac{N_{c}-2}{N_{c}}\right)\right. \\
& \left.\times \sqrt{\frac{1}{4}-\frac{w}{(1-w)} \frac{\left\langle k_{v}\right\rangle}{\left(\langle k\rangle+\langle l\rangle\left\langle k^{\prime}\right\rangle\right)} \frac{\left(N_{c}-1\right)}{N_{c}}}\right\}+ \\
& +2 w
\end{aligned}
$$

Para que $\eta_{+}^{\sigma}$ e $\eta_{-}^{\sigma}$ sejam estáveis, são necessárias as seguintes desigualdades:

$$
\begin{aligned}
& (1-w) \frac{\left(\langle k\rangle+\langle l\rangle\left\langle k^{\prime}\right\rangle\right)}{\left\langle k_{v}\right\rangle}\left(\frac{N_{c}}{N_{c}-1}\right) \times \\
& \times\left\{-\frac{1}{2}-\left(\frac{N_{c}-2}{N_{c}}\right) \sqrt{\frac{1}{4}-\frac{w}{(1-w)} \frac{\left\langle k_{v}\right\rangle}{\left(\langle k\rangle+\langle l\rangle\left\langle k^{\prime}\right\rangle\right)} \frac{\left(N_{c}-1\right)}{N_{c}}}\right\}+ \\
& +2 w<0 \\
& (1-w) \frac{\left(\langle k\rangle+\langle l\rangle\left\langle k^{\prime}\right\rangle\right)}{\left\langle k_{v}\right\rangle}\left(\frac{N_{c}}{N_{c}-1}\right) \times \\
& \times\left\{-\frac{1}{2}+\left(\frac{N_{c}-2}{N_{c}}\right) \sqrt{\frac{1}{4}-\frac{w}{(1-w)} \frac{\left\langle k_{v}\right\rangle}{\left(\langle k\rangle+\langle l\rangle\left\langle k^{\prime}\right\rangle\right)} \frac{\left(N_{c}-1\right)}{N_{c}}}\right\}+ \\
& +2 w<0
\end{aligned}
$$


Ou seja:

$$
\begin{aligned}
& \left(\frac{N_{c}-2}{N_{c}}\right) \sqrt{\frac{1}{4}-\frac{w}{(1-w)} \frac{\left\langle k_{v}\right\rangle}{\left(\langle k\rangle+\langle l\rangle\left\langle k^{\prime}\right\rangle\right)} \frac{\left(N_{c}-1\right)}{N_{c}}}> \\
& >-\frac{1}{2}+\frac{2 w}{(1-w)} \frac{\left\langle k_{v}\right\rangle}{\left(\langle k\rangle+\langle l\rangle\left\langle k^{\prime}\right\rangle\right)}\left(\frac{N_{c}-1}{N_{c}}\right) \\
& \left(\frac{N_{c}-2}{N_{c}}\right) \sqrt{\frac{1}{4}-\frac{w}{(1-w)} \frac{\left\langle k_{v}\right\rangle}{\left(\langle k\rangle+\langle l\rangle\left\langle k^{\prime}\right\rangle\right)} \frac{\left(N_{c}-1\right)}{N_{c}}}< \\
& <\frac{1}{2}-\frac{2 w}{(1-w)} \frac{\left\langle k_{v}\right\rangle}{\left(\langle k\rangle+\langle l\rangle\left\langle k^{\prime}\right\rangle\right)}\left(\frac{N_{c}-1}{N_{c}}\right)= \\
& =2\left[\frac{1}{4}-\frac{w}{(1-w)} \frac{\left\langle k_{v}\right\rangle}{\left(\langle k\rangle+\langle l\rangle\left\langle k^{\prime}\right\rangle\right)}\left(\frac{N_{c}-1}{N_{c}}\right)\right]
\end{aligned}
$$

É fácil mostrar que a inequação (3.7) é satisfeita para todos os valores de w. Da mesma forma, um simples desenvolvimento algébrico mostra que (3.8) é satisfeita por $w<\tilde{w}$.

Assim, podemos apresentar os estados estacionários como segue:

$$
\begin{gathered}
\eta^{0}=0 \\
\eta_{0}^{\sigma}=\frac{1}{N_{c}} \\
\eta_{+}^{\sigma}=\frac{1}{2}+\sqrt{\frac{1}{4}-\frac{w}{(1-w)} \frac{\left\langle k_{v}\right\rangle}{\left(\langle k\rangle+\langle l\rangle\left\langle k^{\prime}\right\rangle\right)} \frac{\left(N_{c}-1\right)}{N_{c}}} \\
\eta_{-}^{\sigma}=\frac{1}{2}-\sqrt{\frac{1}{4}-\frac{w}{(1-w)} \frac{\left\langle k_{v}\right\rangle}{\left(\langle k\rangle+\langle l\rangle\left\langle k^{\prime}\right\rangle\right)} \frac{\left(N_{c}-1\right)}{N_{c}}} \\
0 \leq w<\tilde{w} \quad \Rightarrow \quad \eta_{-}^{\sigma}<\eta_{0}^{\sigma}<\eta_{+}^{\sigma}, \text { com } \eta_{0}^{\sigma} \text { estável } \\
\tilde{w}<w<w_{t} \Rightarrow \eta_{0}^{\sigma}<\eta_{-}^{\sigma}<\eta_{+}^{\sigma}, \text { com } \eta_{-}^{\sigma} \text { estável } \\
w>w_{t} \quad \Rightarrow \quad \text { apenas } \eta_{0}^{\sigma} \text { é estacionária (e estável) } \\
\tilde{w} \equiv\left[1+\frac{\left\langle k_{v}\right\rangle N_{c}}{\left(\langle k\rangle+\langle l\rangle\left\langle k^{\prime}\right\rangle\right)}\right]^{-1}
\end{gathered}
$$




$$
w_{t} \equiv\left[1+4 \frac{\left\langle k_{v}\right\rangle}{\left(\langle k\rangle+\langle l\rangle\left\langle k^{\prime}\right\rangle\right)} \frac{\left(N_{c}-1\right)}{N_{c}}\right]^{-1}
$$

Tudo isso pode ser resumido no seguinte diagrama de bifurcação mostrado na Figura 3.6.

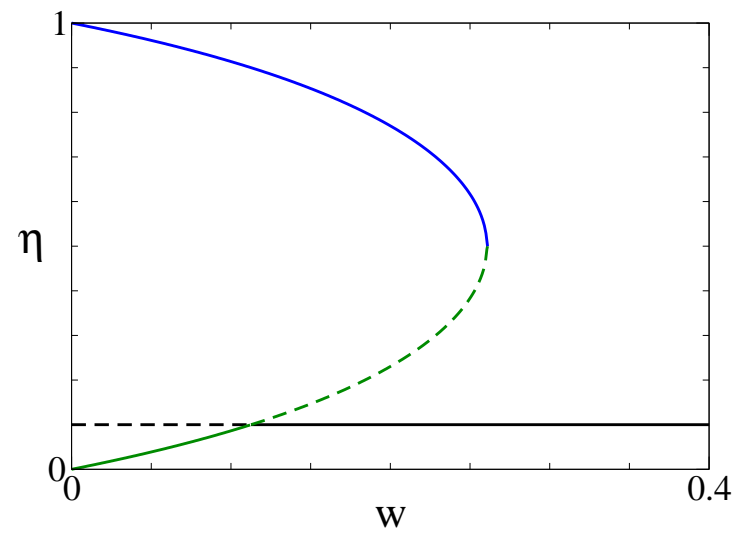

Figura 3.6: Diagrama de bifurcação típico para o Modelo Sznajd Complexo numa aproximação de campo médio. As linhas tracejadas representam soluções instáveis.

O fato da bifurcação ser descontinua sugere a existência de uma transição de fases de primeira ordem entre a fase com um vencedor isolado (a grande maioria dos eleitores com a mesma opinião) e a fase de empate técnico entre todos os candidatos (os candidatos apresentam, em média, a mesma quantidade de votos) com ponto crítico determinado por $w_{t}$. Diante disto, é conveniente definir um parâmetro de ordem para o sistema como $\Psi=\left(\frac{N_{c}}{N_{c}-1}\right)\left(\eta_{+}^{\sigma}-\eta_{0}^{\sigma}\right)$. O comportamento típico do parâmetro de ordem e a transição de fases estão indicados na Figura 3.7. 


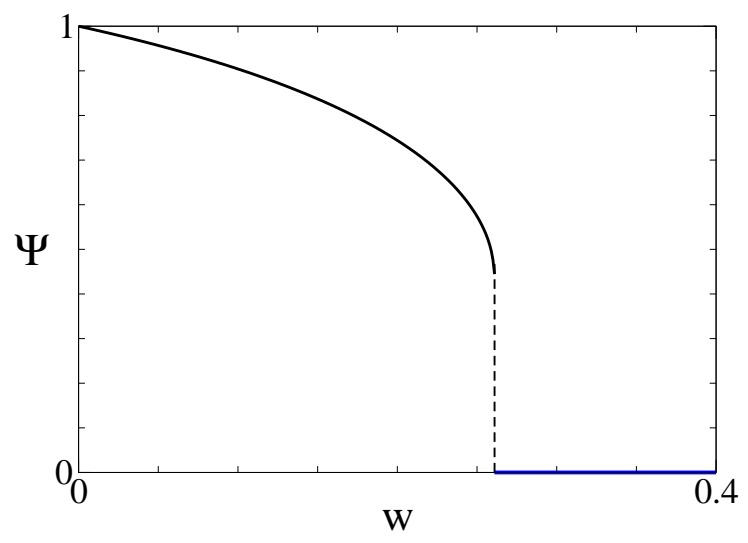

Figura 3.7: Diagrama típico do parâmetro de ordem contra o parâmetro de controle para o Modelo Sznajd Complexo numa aproximação de campo médio.

\subsection{Análise de Casos Limites}

\subsubsection{Campo Nulo}

Se tomarmos as soluções estacionárias a campo nulo $(w \rightarrow 0)$, obtemos:

$$
\left\{\begin{aligned}
\eta^{0} & =0 \\
\eta_{0}^{\sigma} & =\frac{1}{N_{c}} \\
\eta_{+}^{\sigma} & \rightarrow 1 \\
\eta_{-}^{\sigma} & \rightarrow 0
\end{aligned}\right.
$$

Como esperado, recuperamos os resultados para o caso sem ruído anteriormente calculado. Além disso, os resultados concordam com o diagrama de bifurcação da Figura 1 no caso particular do eixo w=0. 


\subsubsection{Campo Máximo}

No caso de $w \rightarrow 1$ :

$$
\left\{\begin{aligned}
\eta^{0} & =0 \\
\eta_{0}^{\sigma} & =\frac{1}{N_{c}} \\
\eta_{+}^{\sigma} & \rightarrow i \infty \quad \text { (não aceitável fisicamente) } \\
\eta_{-}^{\sigma} \rightarrow-i \infty & \text { (não aceitável fisicamente) }
\end{aligned}\right.
$$

Nesta situação, o termo de ruído domina o termo que controla a dinâmica convencional, estabelecendo um estado de equiprobabilidade entre os candidatos (empate técnico).

\subsubsection{Campo Fraco}

Numa aproximação de campo fraco, ou seja, $w \ll 1$, temos:

$$
\begin{aligned}
\eta^{0} & =0 \quad(\text { estável }) \\
\eta_{0}^{\sigma} & =\frac{1}{N_{c}} \quad(\text { instável }) \\
\eta_{+}^{\sigma} & \approx 1-2 \alpha \frac{\left(N_{c}-1\right)}{N_{c}} w \\
\eta_{-}^{\sigma} & \approx 2 \alpha \frac{\left(N_{c}-1\right)}{N_{c}} w \\
\alpha & \equiv \frac{\left\langle k_{v}\right\rangle}{\langle k\rangle+\langle l\rangle\left\langle k^{\prime}\right\rangle}
\end{aligned}
$$

1. Esta simples análise revela que $\eta_{+}^{\sigma}$ representa o candidato majoritário, enquanto que $\eta_{-}^{\sigma}$ representa a totalização de todos os demais candidatos, pois $\eta^{0}+\eta_{+}^{\sigma}+\eta_{-}^{\sigma}=1$.

2. A interpretação física de $\eta_{-}^{\sigma}$ é consequëncia imediata da hipótese de campo médio assumida para o valor de $\eta^{\sigma^{\prime}}$. 
3. Nesta aproximação, a solução estácionária que representa o candidato majoritário se afasta linearmente do estado de consenso com o aumento do ruído $(\sim w)$.

4. O estado assumido pelo sistema é $\left(\eta^{0}=0, \eta_{+}^{\sigma_{v}}, \eta_{-}^{\sigma}\right)$, onde $\eta_{-}^{\sigma}$ representa a totalização da probabilidade de intenções de votos dos outros candidatos diferentes do candidato majoritário $\sigma_{v}$.

5. No caso extremo $\mathrm{w}=0$, obtemos o resultado a campo nulo, como esperado.

\subsubsection{Campo Forte}

Para a situação de campos fortes, ou seja, $w \lesssim 1$, temos:

$$
\begin{aligned}
& \eta^{0}=0 \quad(\text { estável }) \\
& \eta_{0}^{\sigma}=\frac{1}{N_{c}} \quad(\text { estável }) \\
& \eta_{+}^{\sigma} \approx i \sqrt{\alpha \frac{\left(N_{c}-1\right)}{N_{c}} \frac{1}{(1-w)}} \quad \text { (não aceitável fisicamente) } \\
& \eta_{-}^{\sigma} \approx-i \sqrt{\alpha \frac{\left(N_{c}-1\right)}{N_{c}} \frac{1}{(1-w)}} \quad \text { (não aceitável fisicamente) }
\end{aligned}
$$

1. Esta situação leva o sistema, necessariamente, ao estado de empate técnico entre todos os candidatos.

2. No caso extremo $\mathrm{w}=1$, obtemos o resultado para campo forte, como esperado.

3. Os estados estacionários não aceitáveis fisicamente (soluções complexas) divergem como $(1-w)^{-\frac{1}{2}}$.

4. É interessante analisarmos a situação numa escala intermediária para valores de $w$, em que o estado estacionário $\eta_{0}^{\sigma}$ passa a ser 
estável, introduzindo complicações. Esta análise será feita na próxima seção, quando tratarmos da interpretação física do parâmetro $\tilde{w}$.

\subsection{Interpretação Física de $\tilde{w}$ e $w_{t}$}

Numa escala intermediária para valores de $\mathrm{w}\left(\tilde{w}<w<w_{t}\right)$, as soluções estacionárias estáveis são:

$$
\begin{aligned}
\eta^{0} & =0 \\
\eta_{0}^{\sigma} & =\frac{1}{N_{c}} \\
\eta_{+}^{\sigma} & =\frac{1}{2}+\sqrt{\frac{1}{4}-\frac{w}{(1-w)} \frac{\left\langle k_{v}\right\rangle}{\left(\langle k\rangle+\langle l\rangle\left\langle k^{\prime}\right\rangle\right)} \frac{\left(N_{c}-1\right)}{N_{c}}}
\end{aligned}
$$

É possível ao sistema somente um dos seguintes estados:

1. $\left(\eta^{0}=0, \eta^{\sigma_{v}}, \eta_{0}^{\sigma}=\frac{1}{N_{c}}\right), \forall \sigma \neq 0, \sigma_{v}$.

2. $\left(\eta^{0}=0, \eta_{0}^{\sigma}=\frac{1}{N_{c}}\right), \forall \sigma \neq 0$.

Ou seja, não é possível garantir um vencedor isolado para campos tais que $\tilde{w}<w<w_{t}$. Pode ocorrer tanto a situação de vitória isolada quanto a de empate técnico, diferentemente das situações para campos extremos anteriormente discutidas, onde apenas uma das situações prevalecia.

Perceba ainda que o estado (1) apresenta problemáticas quanto a condição de normalização, pois não é possível que todos os valores de $w$ pertencentes ao intervalo $\left(\tilde{w}, w_{t}\right)$ satisfaçam a equação $\eta^{\sigma_{v}}+\eta_{0}^{\sigma}=1$, como é evidente ao analisarmos o diagrama de bifurcação neste intervalo. Esta situação evidencia que a aproximação de campo médio deve ser interpretada, na pior das hipóteses, como uma boa estimativa de 
limitante (superior ou inferior, dependendo do caso) aos possíveis valores assumidos por $\eta^{\sigma}$. Resultados analíticos a serem apresentados no próximo capítulo corroboram essa interpretação.

Agora, um importante questionamento a ser levantado é quão fraco deve ser o campo para que a vitória isolada de um candidato permaneça garantida. É facil perceber que para campos tais que $0<w<\tilde{w}$ a vitória isolada de um candidato é garantida, ou seja, o parâmetro $\tilde{w}$ define a possibilidade ou não de vitória isolada de um candidato.

E o que dizer sobre o parâmetro $w_{t}$ ?

Como $\eta_{+}^{\sigma}$ e $\eta_{-}^{\sigma}$ não são definidas matematicamente no intervalo $w_{t}<w<1$, a única solução possível ao sistema é a correspondente a equiprobabilidade na escolha dos candidatos, ou seja, $\left(\eta^{0}=\right.$ $\left.0, \eta^{\sigma}=\frac{1}{N_{c}}\right), \forall \sigma \neq 0$. De maneira que, neste intervalo, é impossível que qualquer candidato vença, tampouco com vitória isolada. É imediato, então, que o parâmetro $w_{t}$ controla a possibilidade de vitória ou não de pelo menos um candidato, independentemente de ser isolada ou não.

Tudo isso pode ser resumido no seguinte diagrama:

$$
\begin{array}{ccc}
0 \leq w<\tilde{w} & \rightarrow & \text { certeza de vitória isolada de algum candidato } \\
\tilde{w}<w<w_{t} & \rightarrow & \text { é possível, mas não há certeza de vitória isolada } \\
w>w_{t} & \rightarrow & \text { impossível ocorrer vitória isolada }
\end{array}
$$

Se interpretarmos fisicamente a possibilidade de ocorrer vitória isolada de algum candidato como um estado físico ordenado do sistema e a impossibilidade como um estado físico desordenado, é conveniente definirmos um parâmetro de ordem para esse sistema como $\Psi=\left(\frac{N_{c}}{N_{c}-1}\right)\left(\eta_{+}^{\sigma}-\eta_{0}^{\sigma}\right)$. O ponto $w_{t}$ corresponderia ao ponto crítico da transição de fases.

É importante enfatizar que a fase ordenada representa o estado do sistema que existe a possibilidade, não a garantia, de vitória isolada de 
algum candidato. A garantia fica a critério do parâmetro $\tilde{w}$.

$$
\begin{aligned}
& 0 \leq w<w_{t} \rightarrow \Psi \neq 0 \rightarrow \text { é possível ocorrer vitória isolada } \\
& w_{t}<w<1 \rightarrow \Psi=0 \rightarrow \text { impossível ocorrer vitória isolada }
\end{aligned}
$$

Para reforçar a interpretação física de $\tilde{w}$, perceba que na situação limite $N_{c} \gg 1$, temos $\tilde{w} \ll 1$, corroborando com a idéia de que é pouco provável que algum candidato se destaque dentre uma imensidão de outros e vença isoladamente. $N c \gg 1$ pode ser interpretado como uma situação altamente competitiva entre os candidatos.

\subsection{Reconhecendo a Possibilidade de uma Transi- ção de Fases de Segunda Ordem}

É interessante considerarmos o caso particular $N_{c}=2$, pois, nesta situação, é evidente que o parâmetro de ordem será uma função continua do parâmetro de controle, sugerindo a possibilidade de uma transição de fase de segunda ordem, ao invés de uma transição de primeira ordem. Esse caso particular é especialmente interessante por ser capaz de modelar eleições majoritárias e plebiscitos.

O ponto crítico da transição coincide com o parâmetro $\tilde{w}$. Desta forma a fase ordenada representaria não só a possibilidade mas também a certeza de vitória isolada, enquanto a fase desordenada continuaria representando a impossibilidade de vitória isolada.

$$
w_{t}=\left[1+4 \frac{\left\langle k_{v}\right\rangle}{\left(\langle k\rangle+\langle l\rangle\left\langle k^{\prime}\right\rangle\right)} \frac{(2-1)}{2}\right]^{-1}=\left[1+\frac{2\left\langle k_{v}\right\rangle}{\left(\langle k\rangle+\langle l\rangle\left\langle k^{\prime}\right\rangle\right)}\right]^{-1}=\tilde{w}
$$

Destacamos que apenas para $N_{c}=2$ o parâmetro de ordem varia continuamente (levantando a possibilidade de uma transição de se- 
gunda ordem), $\operatorname{com} \tilde{w}=w_{t}$.

$$
\begin{aligned}
{\left[1+\frac{\left\langle k_{v}\right\rangle N_{c}}{\left(\langle k\rangle+\langle l\rangle\left\langle k^{\prime}\right\rangle\right)}\right]^{-1} } & =\left[1+4 \frac{\left\langle k_{v}\right\rangle}{\left(\langle k\rangle+\langle l\rangle\left\langle k^{\prime}\right\rangle\right)} \frac{\left(N_{c}-1\right)}{N_{c}}\right]^{-1} \\
\Rightarrow 1+\frac{\left\langle k_{v}\right\rangle N_{c}}{\left(\langle k\rangle+\langle l\rangle\left\langle k^{\prime}\right\rangle\right)} & =1+4 \frac{\left\langle k_{v}\right\rangle}{\left(\langle k\rangle+\langle l\rangle\left\langle k^{\prime}\right\rangle\right)} \frac{\left(N_{c}-1\right)}{N_{c}} \\
\therefore \quad & N_{c}^{2}-4 N_{c}+4=0
\end{aligned}
$$

O discriminante de (3.9) é nulo e, portanto, apresenta $N_{c}=2$ como única solução.

\subsection{Resultados para um Grafo Completo}

Um grafo completo é a rede natural para cálculos de campo médio. Ela apresenta a peculiar propriedade da igualdade entre o grau médio, o grau médio da primeira vizinhança e o número médio de segundos vizinhos de um vértice $\left(\langle k\rangle=\left\langle k_{v}\right\rangle=\left\langle k^{\prime}\right\rangle\right)$, além de apresentar o máximo valor para o fator de acoplamento entre as vizinhanças, ou seja, $\langle l\rangle=1$. Portanto:

$$
\begin{gathered}
w_{t}=\left[1+4 \frac{\left\langle k_{v}\right\rangle}{\left(\langle k\rangle+\langle l\rangle\left\langle k^{\prime}\right\rangle\right)} \frac{\left(N_{c}-1\right)}{N_{c}}\right]^{-1}=\left[1+2 \frac{\left(N_{c}-1\right)}{N_{c}}\right]^{-1} \\
\tilde{w}=\left[1+\frac{\left\langle k_{v}\right\rangle N_{c}}{\left(\langle k\rangle+\langle l\rangle\left\langle k^{\prime}\right\rangle\right)}\right]^{-1}=\left[1+\frac{N_{c}}{2}\right]^{-1}
\end{gathered}
$$

A seguir, desenvolveremos os cálculos para alguns valores de $N_{c}$ de grande relevância.

$N_{c}=10$ : este caso é particularmente importante para a comparação com os resultados numéricos que serão apresentados no próximo capítulo. 


$$
\begin{gathered}
w_{t}=\left[1+2\left(\frac{9}{10}\right)\right]^{-1}=\frac{5}{14} \approx 0,357 \\
\tilde{w}=\left[1+\frac{10}{2}\right]^{-1}=\frac{1}{6}=0,1666 \ldots
\end{gathered}
$$

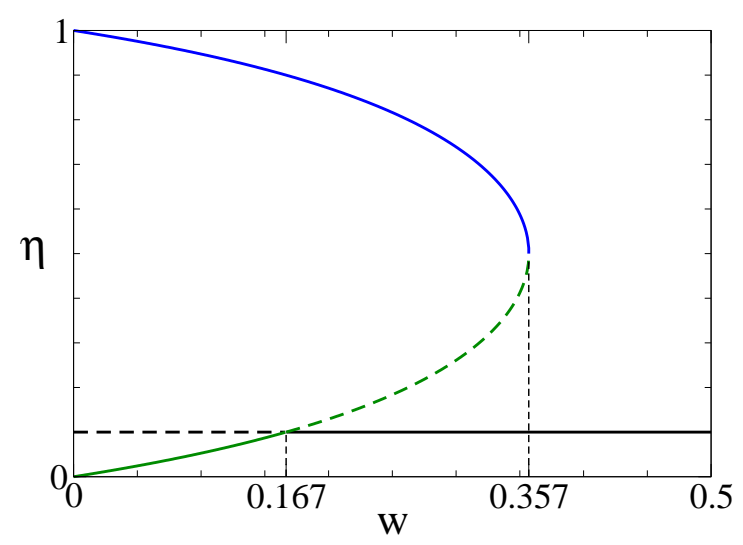

Figura 3.8: Diagrama de bifurcação para o Modelo Sznajd Complexo referente a um grafo completo com 10 candidatos numa aproximação de campo médio. As linhas tracejadas representam soluções instáveis.

$N_{c}=2$ : este caso é particularmente importante por representar uma possível ocorrência de transição de fases de segunda ordem.

$$
\begin{aligned}
& w_{t}=\left[1+2\left(\frac{1}{2}\right)\right]^{-1}=\frac{1}{2}=0,5 \\
& \tilde{w}=\left[1+\frac{2}{2}\right]^{-1}=\frac{1}{2}=0,5=w_{t}
\end{aligned}
$$

Os cálculos sugerem que o valor 0,5 é um limitante superior para os valores de $\tilde{w}$ e $w_{t}$. 


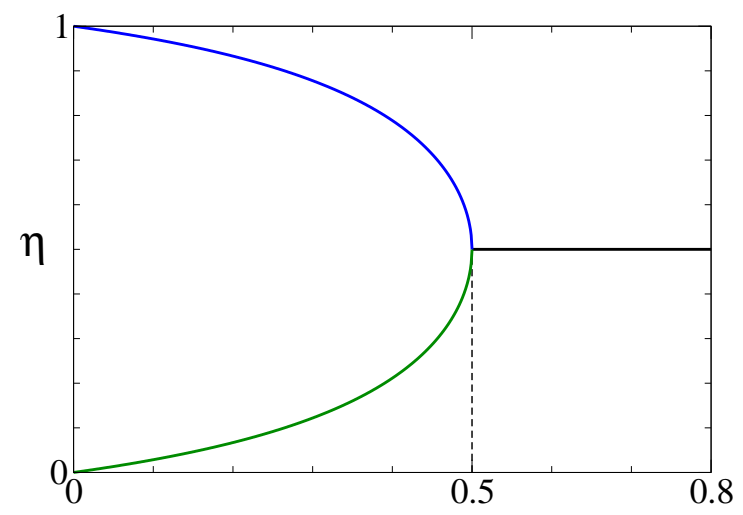

Figura 3.9: Diagrama de bifurcação para o Modelo Sznajd Complexo referente a um grafo completo com 2 candidatos numa aproximação de campo médio. As linhas tracejadas representam soluções instáveis.

$N_{c} \gg 1$ : este caso corresponde a uma situação de alta competitividade entre os candidatos.

$$
\begin{gathered}
w_{t} \rightarrow[1+2]^{-1}=\frac{1}{3}=0,333 \ldots \\
\tilde{w} \rightarrow\left[\frac{N_{c}}{2}\right]^{-1} \rightarrow \frac{2}{N_{c}} \rightarrow 0
\end{gathered}
$$

O valor $0,333 \ldots$ assumido por $w_{t}$ é, provavelmente, o valor mais baixo que este parâmetro pode assumir, corroborando a expectativa de que, numa eleição bastante competitiva, a transição para a fase desordenada deve acontecer num valor relativamente pequeno de intensidades de ruído. Além disso, $\tilde{w}$ tende a zero, conforme discussão realizada na secção anterior. 


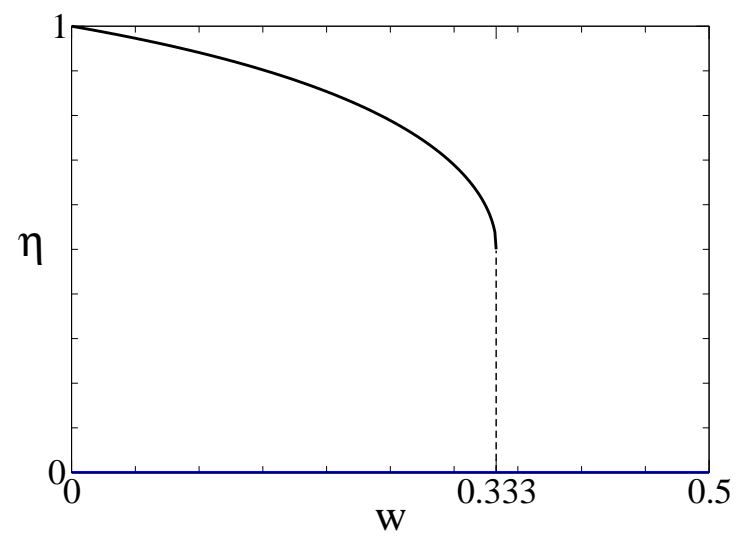

Figura 3.10: Diagrama de bifurcação para o Modelo Sznajd Complexo referente a um grafo completo com alta campetitividade entre os candidatos numa aproximação de campo médio. 


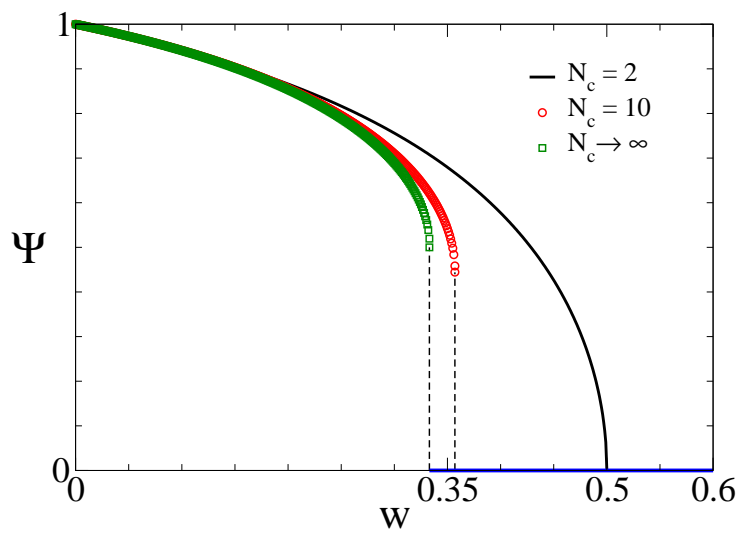

Figura 3.11: Parâmetro de ordem contra o parâmetro de controle para o Modelo Sznajd Complexo referente a um grafo completo numa aproximação de campo médio com diferentes valores para o número de candidatos.

Uma comparação detalhada entre esses casos é apresentada na Tabela 3.1.

\subsection{Resultados para Redes Regulares}

Nesta seção apresentaremos os resultados da aproximação de campo médio para o Modelo Sznajd Complexo para redes regulares em diferentes dimensionalidades. Estes casos ilustram de maneira bastante instrutiva o cálculo dos parâmetros que caracterizam a rede, diferentemente do grafo completo em que os parâmetros são determinados imediatamente, a partir de suas propriedades gerais. 


\subsubsection{Cadeia de Eleitores}

No caso unidimensional $(d=1)$, temos:

$$
\begin{aligned}
& k_{i}=k_{v i}=2, \forall i \Rightarrow\langle k\rangle=\left\langle k_{v}\right\rangle=2 \\
& k_{i}^{\prime}=2, \forall i \Rightarrow\left\langle k^{\prime}\right\rangle=2 \\
& l_{i}=\frac{1}{2}, \forall i \Rightarrow\langle l\rangle=\frac{1}{2}
\end{aligned}
$$

$\therefore$

$$
\alpha_{1 D}=\frac{2}{2+\frac{1}{2} 2}=\frac{2}{3}
$$

Portanto:

$$
\begin{gathered}
w_{t}^{(1 D)}=\left[1+\frac{8}{3} \frac{\left(N_{c}-1\right)}{N_{c}}\right]^{-1} \\
\tilde{w}_{1 D}=\left[1+\frac{2}{3} N_{c}\right]^{-1}
\end{gathered}
$$

De acordo com a discussão anterior, calculemos $\tilde{w}$ e $w_{t}$ para diferentes números de candidatos.

$N_{c}=10:$

$$
\begin{gathered}
w_{t}^{(1 D)}=\left[1+\frac{8}{3} \frac{9}{10}\right]^{-1}=\frac{5}{17} \approx 0,294 \\
\tilde{w}_{1 D}=\left[1+\frac{2}{3} 10\right]^{-1}=\frac{3}{23} \approx 0,130
\end{gathered}
$$




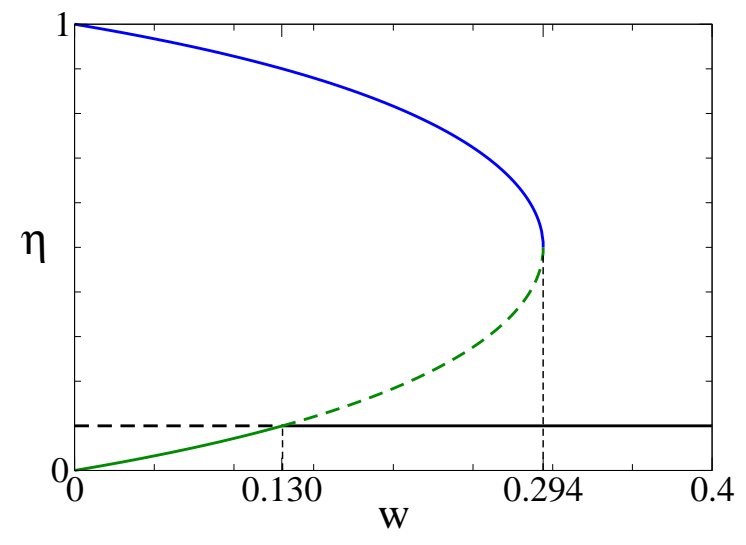

Figura 3.12: Diagrama de bifurcação para o Modelo Sznajd Complexo referente a uma cadeia de eleitores com 10 candidatos numa aproximação de campo médio. As linhas tracejadas representam soluções instáveis.

$$
\begin{gathered}
N_{c}=2: \\
w_{t}^{(1 D)}=\left[1+\frac{8}{3} \frac{1}{2}\right]^{-1}=\frac{3}{7}=0,428 \\
\tilde{w}_{1 D}=\left[1+\frac{2}{3} 2\right]^{-1}=\frac{3}{7}=0,428=w_{t}^{(1 D)}
\end{gathered}
$$




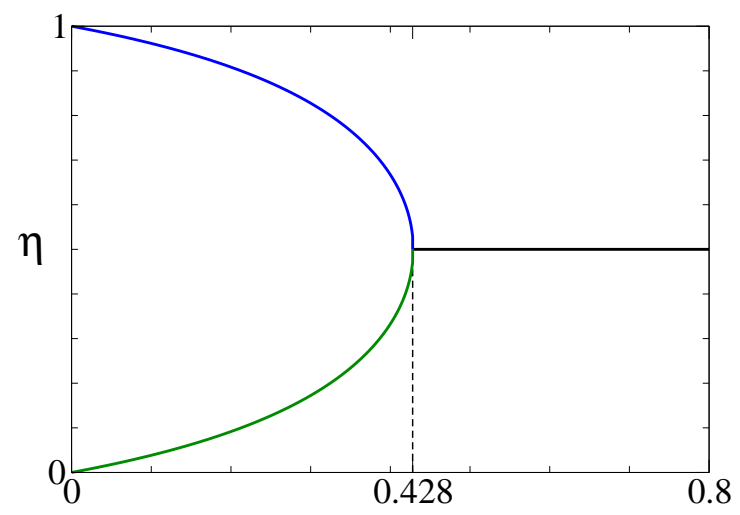

Figura 3.13: Diagrama de bifurcação para o Modelo Sznajd Complexo referente a uma cadeia de eleitores com 2 candidatos numa aproximação de campo médio. As linhas tracejadas representam soluções instáveis.

$$
N_{c} \gg 1:
$$

$$
\begin{gathered}
w_{t}^{(1 D)} \rightarrow\left[1+\frac{8}{3}\right]^{-1}=\frac{3}{11} \approx 0,273 \\
\tilde{w}_{1 D} \rightarrow\left[\frac{2}{3} N_{c}\right]^{-1} \rightarrow \frac{3}{2 N_{c}} \rightarrow 0
\end{gathered}
$$




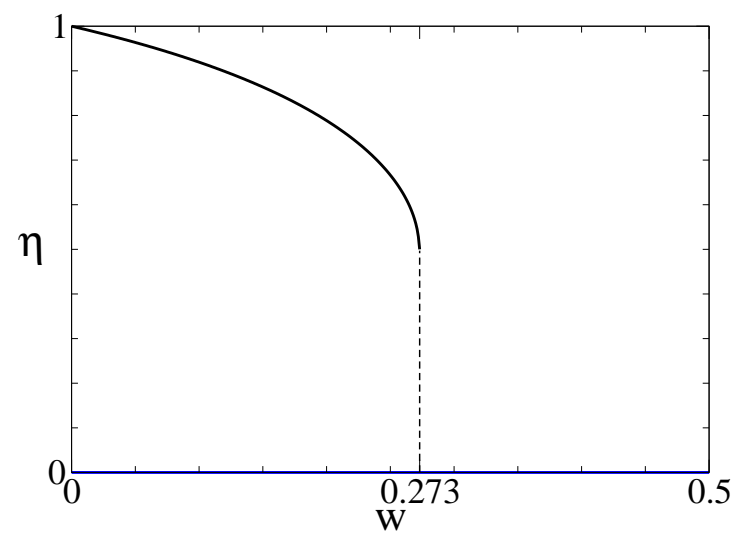

Figura 3.14: Diagrama de bifurcação para o Modelo Sznajd Complexo referente a uma cadeia de eleitores com alta competitividade entre os candidatos numa aproximação de campo médio. 


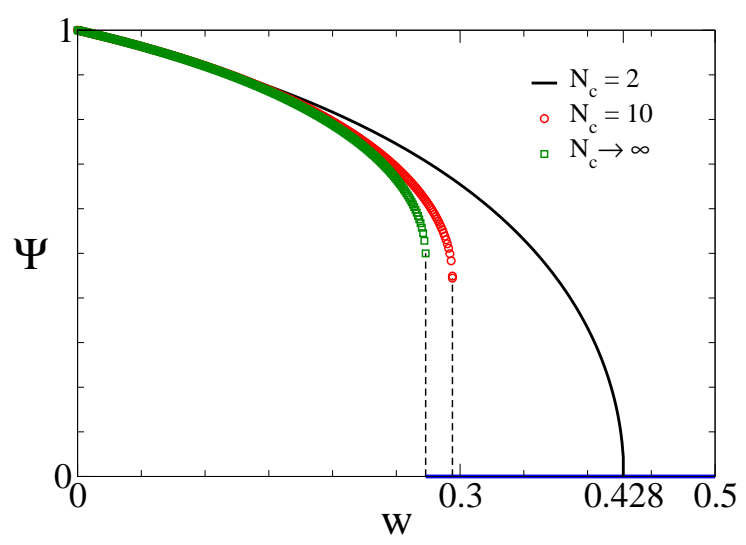

Figura 3.15: Parâmetro de ordem contra o parâmetro de controle para o Modelo Sznajd Complexo referente a uma cadeia de eleitores numa aproximação de campo médio com diferentes valores para o número de candidatos.

Uma comparação detalhada entre esses casos é apresentada na Tabela 3.1.

\subsubsection{Rede Quadrada}

Para o caso bidimensional $(d=2)$, temos:

$$
\begin{gathered}
k_{i}=k_{v i}=4, \forall i \Rightarrow\langle k\rangle=\left\langle k_{v}\right\rangle=4 \\
k_{i}^{\prime}=8, \forall i \Rightarrow\left\langle k^{\prime}\right\rangle=8 \\
l_{i}=\frac{1}{2}, \frac{1}{4} ; \forall i \Rightarrow\langle l\rangle=\frac{4 \frac{1}{2}+4 \frac{1}{4}}{4+4}=\frac{3}{8} \\
\therefore \quad \\
\alpha_{2 D}=\frac{4}{4+\frac{3}{8} 8}=\frac{4}{7}
\end{gathered}
$$


Portanto:

$$
\begin{gathered}
w_{t}^{(2 D)}=\left[1+\frac{16}{7} \frac{\left(N_{c}-1\right)}{N_{c}}\right]^{-1} \\
\tilde{w}_{2 D}=\left[1+\frac{4}{7} N_{c}\right]^{-1}
\end{gathered}
$$

Agora, calculemos $\tilde{w}$ e $w_{t}$ para diferentes números de candidatos.

$N_{c}=10$ :

$$
\begin{gathered}
w_{t}^{(2 D)}=\left[1+\frac{8}{7} \frac{9}{5}\right]^{-1}=\frac{35}{107} \approx 0,327 \\
\tilde{w}_{2 D}=\left[1+\frac{4}{7} 10\right]^{-1}=\frac{7}{47} \approx 0,149
\end{gathered}
$$

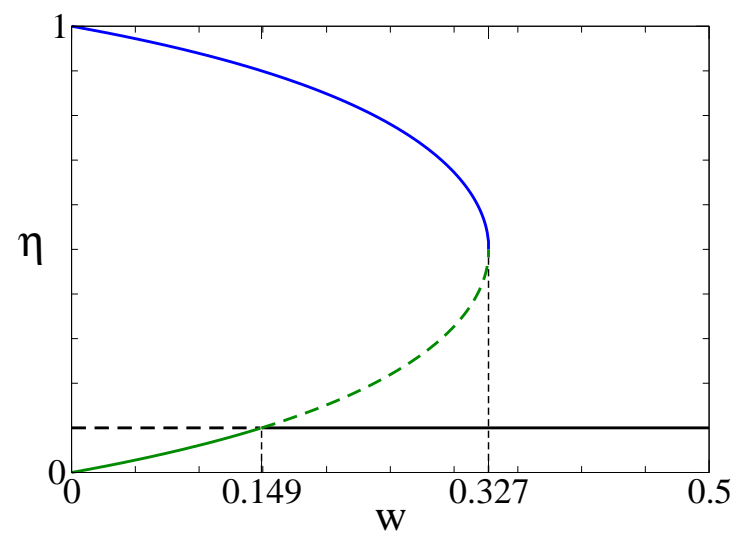

Figura 3.16: Diagrama de bifurcação para o Modelo Sznajd Complexo referente à rede quadrada com 10 candidatos numa aproximação de campo médio. As linhas tracejadas representam soluções instáveis.

$$
N_{c}=2 \text { : }
$$

$$
w_{t}^{(2 D)}=\left[1+4 \frac{4}{7} \frac{1}{2}\right]^{-1}=\frac{7}{15} \approx 0,467
$$




$$
\tilde{w}_{2 D}=\left[1+\frac{4}{7} 2\right]^{-1}=\frac{7}{15}=w_{t}^{(2 D)} \approx 0,467
$$

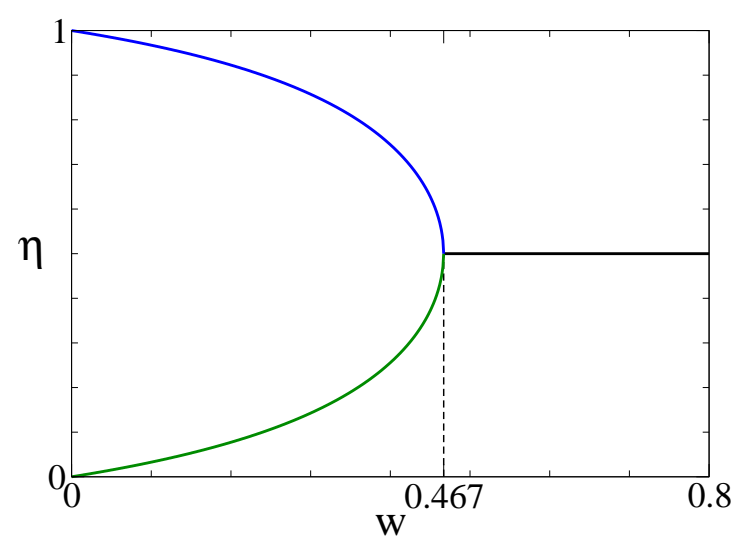

Figura 3.17: Diagrama de bifurcação para o Modelo Sznajd Complexo referente à rede quadrada com 2 candidatos numa aproximação de campo médio. As linhas tracejadas representam soluções instáveis.

$$
\begin{aligned}
N_{c} \gg 1: & w_{t}^{(2 D)} \rightarrow\left[1+4 \frac{4}{7}\right]^{-1}=\frac{7}{23} \approx 0,304 \\
\tilde{w}_{2 D} & \rightarrow\left[\frac{4}{7} N_{c}\right]^{-1} \rightarrow \frac{7}{4 N_{c}} \rightarrow 0
\end{aligned}
$$




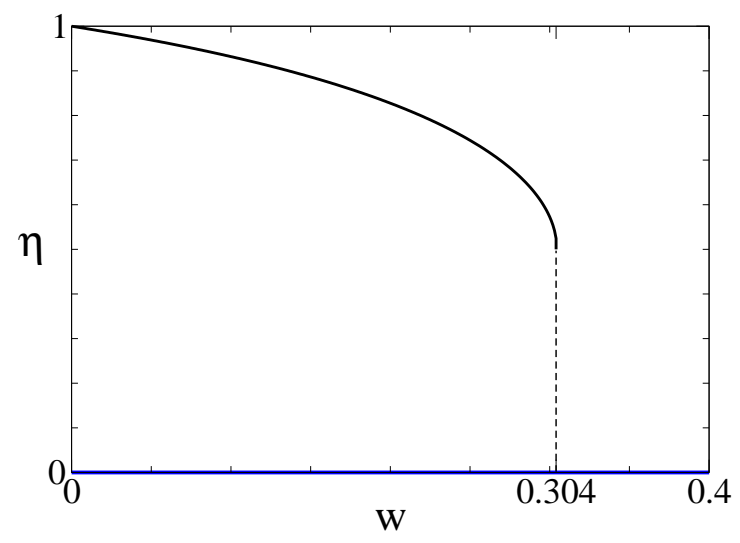

Figura 3.18: Diagrama de bifurcação para o Modelo Sznajd Complexo referente à rede quadrada com alta competitividade entre os candidatos numa aproximação de campo médio. 


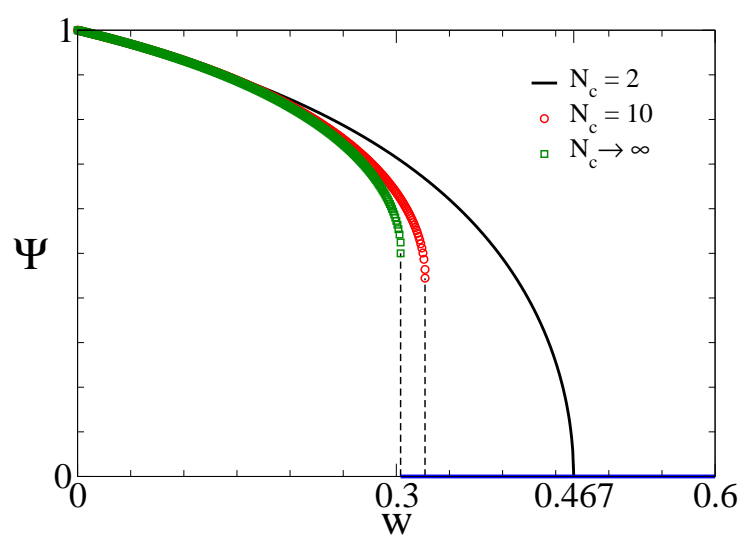

Figura 3.19: Parâmetro de ordem contra o parâmetro de controle para o Modelo Sznajd Complexo referente à rede quadrada numa aproximação de campo médio com diferentes valores para o número de candidatos.

Uma comparação detalhada entre esses casos é apresentada na Tabela 3.1 .

\subsubsection{Rede Cúbica}

Para o caso tridimensional $(d=3)$, temos:

$$
\begin{aligned}
& k_{i}=k_{v i}=6, \forall i \Rightarrow\langle k\rangle=\left\langle k_{v}\right\rangle=6 \\
& k_{i}^{\prime}=18, \forall i \Rightarrow\left\langle k^{\prime}\right\rangle=18 \\
& l_{i}=\frac{1}{3}, \frac{1}{6} ; \forall i \Rightarrow\langle l\rangle=\frac{12 \frac{1}{3}+6 \frac{1}{6}}{12+6}=\frac{5}{18}
\end{aligned}
$$

$\therefore$

$$
\alpha_{3 D}=\frac{6}{6+\frac{5}{18} 18}=\frac{6}{11}
$$


Portanto:

$$
\begin{gathered}
w_{t}^{(3 D)}=\left[1+4 \frac{6}{11} \frac{\left(N_{c}-1\right)}{N_{c}}\right]^{-1} \\
\tilde{w}_{3 D}=\left[1+\frac{6}{11} N_{c}\right]^{-1}
\end{gathered}
$$

Mais uma vez, calcularemos $\tilde{w}$ e $w_{t}$ para $N_{c}=10, N_{c}=2$ e $N_{c} \gg 1$. $N_{c}=10$ :

$$
\begin{aligned}
& w_{t}^{(3 D)}=\left[1+\frac{12}{11} \frac{9}{5}\right]^{-1}=\frac{55}{163} \approx 0,337 \\
& \tilde{w}_{3 D}=\left[1+\frac{6}{11} 10\right]^{-1}=\frac{11}{71} \approx 0,155
\end{aligned}
$$

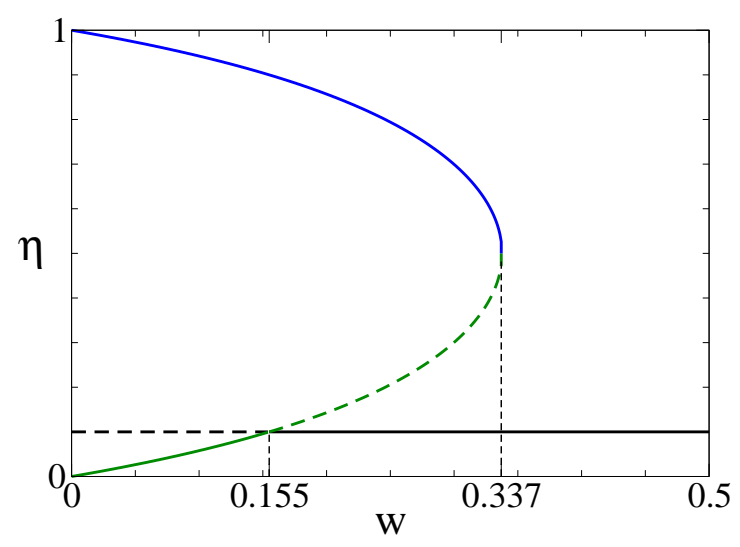

Figura 3.20: Diagrama de bifurcação para o Modelo Sznajd Complexo referente à rede cúbica com 10 candidatos numa aproximação de campo médio. As linhas tracejadas representam soluções instáveis.

$$
N_{c}=2 \text { : }
$$

$$
w_{t}^{(3 D)}=\left[1+4 \frac{6}{11} \frac{1}{2}\right]^{-1}=\frac{11}{23} \approx 0,478
$$




$$
\tilde{w}_{3 D}=\left[1+\frac{6}{11} 2\right]^{-1}=\frac{11}{23}=w_{t}^{(3 D)} \approx 0,478
$$

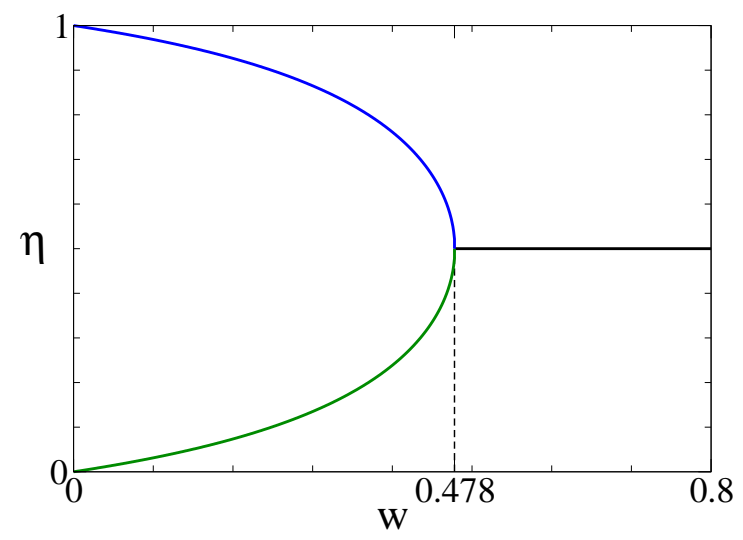

Figura 3.21: Diagrama de bifurcação para o Modelo Sznajd Complexo referente à rede cúbica com 2 candidatos numa aproximação de campo médio. As linhas tracejadas representam soluções instáveis.

$$
\begin{aligned}
N_{c} \gg 1: & w_{t}^{(3 D)} \rightarrow\left[1+4 \frac{6}{11}\right]^{-1}=\frac{11}{35} \approx 0,314 \\
\tilde{w}_{3 D} & \rightarrow\left[\frac{6}{11} N_{c}\right]^{-1} \rightarrow \frac{11}{6 N_{c}} \rightarrow 0
\end{aligned}
$$




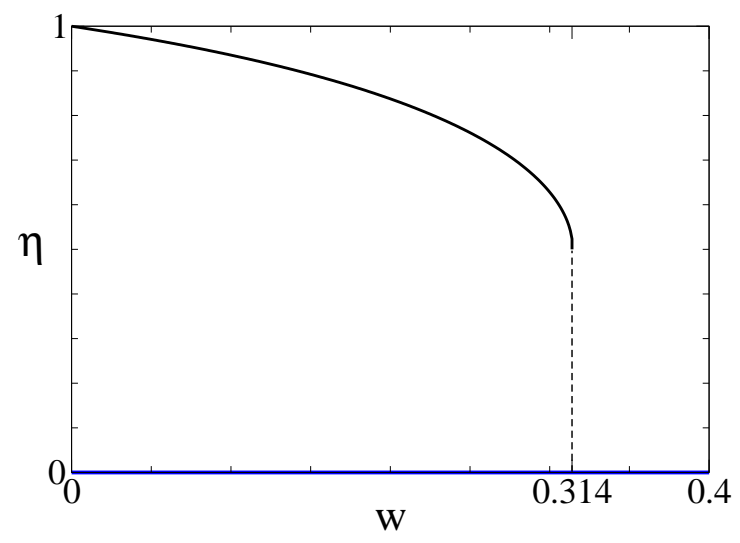

Figura 3.22: Diagrama de bifurcação para o Modelo Sznajd Complexo referente à rede cúbica com alta competitividade entre os candidatos numa aproximação de campo médio. 


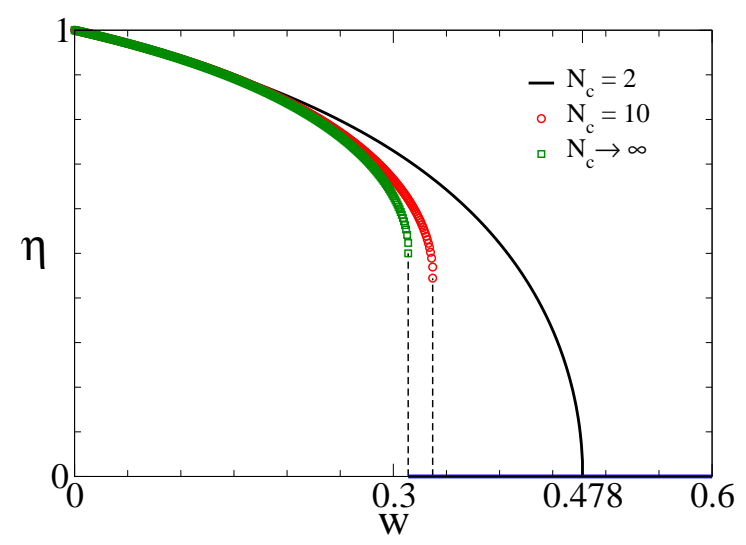

Figura 3.23: Parâmetro de ordem contra o parâmetro de controle para o Modelo Sznajd Complexo referente à rede cúbica numa aproximação de campo médio com diferentes valores para o número de candidatos.

Uma comparação detalhada entre esses casos é apresentada na Tabela 3.1 .

\subsubsection{Limite de Altas Dimensionalidades}

Vejamos agora o caso em que $d \gg 1$, ou seja, o comportamento do modelo para redes de alta dimensionalidade. Podemos realizar um cálculo heurístico com argumentos bastante simplificados para mostrar que o fator $\alpha_{\infty}$ tende a $\frac{1}{2}$. De fato, esperamos que, nestas circuntâncias, $\langle k\rangle$ e $\left\langle k_{v}\right\rangle$ divirgam, enquanto que o produto $\langle l\rangle\left\langle k^{\prime}\right\rangle$ assuma a forma indeterminada $0 \infty$. Entretanto, é razoável que $\langle k\rangle \sim\langle l\rangle\left\langle k^{\prime}\right\rangle$ e portanto:

$$
\alpha_{\infty} \rightarrow \frac{\left\langle k_{v}\right\rangle}{2\langle k\rangle} \rightarrow \frac{1}{2}
$$


$\therefore$

$$
\begin{gathered}
w_{t}^{(\infty)} \rightarrow\left[1+2 \frac{\left(N_{c}-1\right)}{N_{c}}\right]^{-1} \\
\tilde{w}_{\infty} \rightarrow\left[1+\frac{N_{c}}{2}\right]^{-1}
\end{gathered}
$$

Desta forma, percebemos que os resultados para redes regulares no limite de alta dimensionalidade são os mesmos estabelecidos para um grafo completo no limite termodinâmico, o que era esperado, pois, as correlações espaciais são descartadas de forma equivalente nos dois casos. Mais detalhes da comparação entre estas redes são apresentados nas tabelas 3.1 e 3.2 . 


\begin{tabular}{|c|c|c|c|c|}
\hline Rede & Dimensionalidade & - & $\tilde{w}$ & $w_{t}$ \\
\hline \multirow{3}{*}{ Grafo Completo } & \multirow{3}{*}{-} & $N_{c}=2$ & $1 / 2$ & $1 / 2$ \\
\hline & & $N_{c}=10$ & $1 / 6$ & $5 / 14$ \\
\hline & & $N_{c} \gg 1$ & 0 & $1 / 3$ \\
\hline \multirow{12}{*}{ Rede Regular } & \multirow{3}{*}{$d=1$} & $N_{c}=2$ & $3 / 7$ & $3 / 7$ \\
\hline & & $N_{c}=10$ & $3 / 23$ & $5 / 17$ \\
\hline & & $N_{c} \gg 1$ & 0 & $3 / 11$ \\
\hline & \multirow{3}{*}{$d=2$} & $N_{c}=2$ & $7 / 15$ & $7 / 15$ \\
\hline & & $N_{c}=10$ & $7 / 47$ & $35 / 107$ \\
\hline & & $N_{c} \gg 1$ & 0 & $7 / 23$ \\
\hline & \multirow{3}{*}{$d=3$} & $N_{c}=2$ & $11 / 23$ & $11 / 23$ \\
\hline & & $N_{c}=10$ & $11 / 71$ & $55 / 163$ \\
\hline & & $N_{c} \gg 1$ & 0 & $11 / 35$ \\
\hline & \multirow{3}{*}{$d=\infty$} & $N_{c}=2$ & $1 / 2$ & $1 / 2$ \\
\hline & & $N_{c}=10$ & $1 / 6$ & $5 / 14$ \\
\hline & & $N_{c} \gg 1$ & 0 & $1 / 3$ \\
\hline
\end{tabular}

Tabela 3.1: Resumo dos principais resultados obtidos para diferentes redes e numa aproximação de campo médio para o Modelo Sznajd Complexo.

\begin{tabular}{|c|c|c|c|c|c|c|c|}
\hline Rede & - & $\langle k\rangle$ & $\left\langle k_{v}\right\rangle$ & $\left\langle k^{\prime}\right\rangle$ & $\langle l\rangle$ & $\langle l\rangle\left\langle k^{\prime}\right\rangle$ & $\alpha$ \\
\hline \multirow{2}{*}{$\begin{array}{c}\text { Grafo } \\
\text { Completo }\end{array}$} & $N<\infty$ & $N-1$ & $N-1$ & $N-1$ & 1 & $N-1$ & $1 / 2$ \\
\cline { 2 - 8 } & $N=\infty$ & $\infty$ & $\infty$ & $\infty$ & 1 & $\infty$ & $1 / 2$ \\
\hline \multirow{3}{*}{$\begin{array}{c}\text { Rede } \\
\text { Regular }\end{array}$} & $d=1$ & 2 & 2 & 2 & $1 / 2$ & 1 & $2 / 3$ \\
\cline { 2 - 8 } & $d=2$ & 4 & 4 & 8 & $3 / 8$ & 3 & $4 / 7$ \\
\cline { 2 - 8 } & $d=3$ & 6 & 6 & 18 & $5 / 18$ & 5 & $6 / 11$ \\
\cline { 2 - 8 } & $d=\infty$ & $\infty$ & $\infty$ & $\infty$ & 0 & $\infty$ & $1 / 2$ \\
\hline
\end{tabular}

Tabela 3.2: Valores assumidos pelos parâmetros médios que caracterizam as diferentes redes abordadas numa aproximação de campo médio para o Modelo Sznajd Complexo. 


\section{Capítulo 4}

\section{Simulações de Monte Carlo}

Neste capítulo, reproduziremos as principais propriedades dos estados estacionários do Modelo Sznajd Complexo, através de simulações de Monte Carlo numa rede livre de escala do tipo Barabási-Albert. Simulações em redes regulares e em grafos completos também serão apresentadas. Elas mostram que o estado estacionário do modelo nestas redes menos realistas é bastante promissor na explicação de muitos aspectos relevantes da dinâmica da formação e propagação de opiniões.

Num primeiro momento, pretendemos estabelecer sob o ponto de vista numérico algumas definições previamente exploradas e sistematizar os prodedimentos realizados para o caso particular da rede de Barabási-Albert. A análise numérica de redes regulares será postergada para as seções seguintes e será usada como um teste dos algoritmos desenvolvidos por apresentar valores óbvios para esses parâmetros ou calculáveis de forma trivial. Finalmente, o diagrama de bifurcação e o parâmetro de ordem associados à dinâmica do modelo com ruído serão abordados numericamente para a rede de Barabási-Albert e confrontados com resultados analíticos estabelecidos no capítulo 3. 


\subsection{Procedimento Numérico}

Para as diferentes simulações realizadas, sempre utilizamos uma rede de Barabási-Albert com $N_{0}=6$ vértices iniciais, conectividade mínima $m=5$ arestas por vértice, $N_{c}=10$ candidatos e $N=10^{4}$ eleitores $^{1}$. As condições iniciais do sistema também foram padronizadas, com todos os sítios da rede indecisos, a menos de $N_{c}$ sítios da rede escolhidos aleatoriamente, correspondendo ao processo de semeadura.

Utilizamos ainda a Linguagem de Programação C para desenvolvermos os algoritmos de Monte Carlo utilizados na simulação do modelo e o cluster ABAX do Grupo de Mecânica Estatística do Departamento de Física Geral do IFUSP para a realização dos cálculos. O cluster possui um servidor com sistema operacional Debian-Linux, 14 nós com processadores Intel Core 2 Quad Q9300 e 36GB de memória RAM distribuídas em 8GB para o servidor e 2GB para cada nó.

\subsubsection{Representações Numéricas da Rede}

Para desenvolvermos um algoritmo que simule um processo dinâmico é necessário introduzir numericamente a idéia de tempo e uma unidade de medida conveniente. Utilizaremos, então, a unidade Tempo de Monte Carlo (TMC) para designar o intervalo de tempo em que o algoritmo realiza $\mathrm{N}$ iterações da dinâmica, onde cada iteração corresponde à aplicação de uma das regras do modelo. Cada iteração está associada a um instante particular do processo que denominamos Passo de Monte Carlo (PMC). Portanto, 1 TMC equivale a N PMC, e pode ser interpretado fisicamente como o tempo necessário para que todos os sítios da rede sejam atualizados uma única vez, visto que, ao considerarmos uma grande quantidade de realizações, teremos, em média, cada

\footnotetext{
${ }^{1}$ Em alguns casos, variou-se o tamanho da rede entre $10^{3}$ e $10^{4}$ eleitores.
} 
eleitor participando o mesmo número de vezes da dinâmica neste intervalo de tempo. É interessante comentar que sistemas com tamanhos diferentes (número de eleitores diferentes) estarão associados a intervalos de tempo de simulação diferentes, apesar de serem medidos pela mesma unidade de medida.

Quanto ao tratamento da rede, a abordagem numérica requer que cada um de seus sítios sejam numerados com inteiros entre 0 e $N-1$, permitindo sua representação através de um ponteiro inteiro ** $\mathrm{M}$ de ordem $\mathrm{a}(\mathrm{m}, \mathrm{N}) \mathrm{x} 2$, onde $\mathrm{a}(\mathrm{m}, \mathrm{N})$ é o numero de arestas para uma rede com $\mathrm{N}$ sítios e conectividade mínima m. Esta representação será denominada Ponteiro da Representação de Arestas ou, simplesmente, Representação de Arestas. No caso da rede de Barabási-Albert, o processo de construção da rede é detalhado no apêndice A.

As opiniões que um sítio pode assumir também são numeradas com inteiros de 0 a $N_{c}$, onde 0 representa a indecisão e os demais números designam os candidatos, ou seja, $\sigma_{i} \in\left\{0, \ldots, N_{c}\right\}, \forall i \in\{0, \ldots, N-1\}$. Portanto $\sigma_{2}=5$ indica que o sítio 2 escolheu o candidato 5 , por exemplo.

Desta forma, os sítios da rede podem ser identificados como as componentes de um ponteiro inteiro ${ }^{*} \mathrm{Op}$, que assume valores entre 0 e $N_{c}$, constituindo uma nova representação que denominaremos Ponteiro de Opiniões. Esta representação é interpretada fisicamente como uma particular configuração que a rede pode assumir em um determinando instante do processo de evolução das opiniões. A dinâmica será implementada no programa quando permitirmos que o ponteiro de opiniões varie no tempo. Um exemplo típico de saída pode ser visto no apêndice B.

Temos ainda uma terceira forma de representação que denominamos Representação de Vizinhos, associada numericamente ao ponteiro **A. Esta representação adquire caráter especial, pois o trata- 
mento da dinâmica envolve interações entre um sítio e sua vizinhança e a Representação de Arestas não é conveniente para tratar estas interações. No apêndice $\mathrm{C}$ detalhamos seu processo de construção.

Terminada as etapas de construção e reconhecimento da rede, é necessário introduzir as regras da dinâmica no programa. As regras já foram apresentadas e detalharemos sua construção na próxima subseção. Neste estágio, é interessante destacar ainda uma última representação bastante útil para tratar a evolução das opiniões de cada sítio, denominada Representação de Número de Votos, feita através do ponteiro *Nvotos. Esta representação é particularmente útil na determinação das séries temporais que descrevem a dinâmica do modelo, pois as entradas Nvotos[i](t) determinam a configuração do sistema quanto a distribuição de votos por candidato em função do tempo. É através desta representação que calculamos o parâmetro de ordem e determinamos o diagrama de fases do modelo. Novamente, exemplos são apresentados no apêndice D.

Maiores detalhes sobre a estrutura geral e funcionamento dos programas estão apresentados nos apêndices F e G.

\subsubsection{Implementando a Dinâmica}

A implementação da dinâmica do Modelo Sznajd Complexo segue imediatamente das regras de convencimento associadas ao modelo. As principais etapas de sua construção numérica estão dispostas na Tabela $4.1^{2}$.

\footnotetext{
${ }^{2}$ Utilizamos a notação utilizada na Linguagem $\mathrm{C}$ para os símbolos matemáticos, ou seja, $=\rightarrow==\mathrm{e} \neq \rightarrow$ ! $=$
} 


\begin{tabular}{|c|c|c|c|}
\hline$O p[i](t)$ & $O p[j](t)$ & - & Resultado \\
\hline$==0$ & - & & Nada ocorre \\
\hline \multirow{3}{*}{$!=0$} & $==0$ & & $O p[j](t+1)=O p[i](t)$ \\
\hline & \multirow[t]{2}{*}{$!=0$} & $O p[j](t)==O p[i](t)$ & Assediam seus vizinhos \\
\hline & & $O p[j](t) !=O p[i](t)$ & Nada ocorre \\
\hline
\end{tabular}

Tabela 4.1: Algoritmo empregado na implementação da dinâmica de convencimentos do Modelo Sznajd Complexo.

A introdução de ruído na dinâmica é mais delicada e se dá através de duas variáveis do tipo float, denominadas wsort e noise. A variável pseudo-aleatória wsort é responsável por sortear equiprobabilisticamente números reais pertencentes ao intervalo $[0,1]$; um número menor do que o valor de noise indica que a intensidade do ruído é suficientemente grande para ser capaz de alterar a dinâmica convencional do modelo, fazendo que o sítio escolha um candidato - de forma aleatória e equiprobabilística - por intermédio de uma terceira variável pseudoaleatória inteira ${ }^{3}$. Desta forma identificamos o valor de noise como o correspondente numérico da probabilidade $w$ do ruído alterar a dinâmica convencional. Destacamos o algoritmo do programa no caso com ruído na Tabela 4.2 .

\footnotetext{
${ }^{3}$ Utilizamos a função rand, definida na biblioteca stdlib da Linguagem C, como o gerador de números aleatórios nas simulações.
} 


\begin{tabular}{|c|c|c|c|c|}
\hline wsort & $O p[i](t)$ & $O p[j](t)$ & - & Resultado \\
\hline \multirow{4}{*}{$>$ noise } & $==0$ & - & & Nada ocorre \\
\hline & \multirow{3}{*}{$!=0$} & $==0$ & & $O p[j](t+1)=O p[i](t)$ \\
\hline & & \multirow[t]{2}{*}{$!=0$} & $O p[j]==O p[i]$ & Assediam seus vizinhos \\
\hline & & & $O p[j] !=O p[i]$ & Nada ocorre \\
\hline$<$ noise & - & - & & Candidato aleatório \\
\hline
\end{tabular}

Tabela 4.2: Principais etapas na construção numérica da dinâmica de convencimentos do Modelo Sznajd Complexo com Ruído.

É importante destacar que apesar do caso da dinâmica sem ruído ser um caso particular do caso com ruído (para noise $=0$ ), o tratamento numérico nos dois casos é bastante diferenciado. A escolha do tempo de simulação, as grandezas a serem calculadas e o comportamento mais rico do caso com ruído, exibindo transição de fases, são os principais fatores que determinam esta diferenciação.

No apêndice E, apresentaremos uma série de ilustrações que acompanham passo a passo o algoritmo, tanto no caso do ruído como no caso sem ruído, e que esperamos possam ajudar em seu entendimento.

Maiores detalhes sobre a estrutura geral e funcionamento da dinâmica nas simulações estão apresentados nos apêndices F e G.

\subsubsection{Cálculo dos Parâmetros Médios da Rede}

Nesta seção pretendemos tornar mais claro os conceitos de grau médio dos segundos vizinhos de um sítio, $\left\langle k_{v}\right\rangle$, e fator de acoplamento entre as vizinhanças, $\langle l\rangle$, introduzidos no capítulo 3 , por serem mais sutis e pouco frequentes na literatura.

O cálculo destes parâmetros para as redes regulares é imediato, conforme fizemos no capítulo anterior. Entretanto, para a rede de Barabási-Albert, cálculos analíticos são bem mais complicados e ne- 
cessitamos de um algoritmo numérico para que, sobre um ensemble satisfatório de simulações, possamos estimá-los. Neste trabalho, consideramos um ensemble de 100 realizações da simulação para os cálculos.

Para o que segue, consideremos uma rede de Barabási-Albert com $N=8$ vértices e conectividade mínima $m=2$ arestas por vértice de acordo com a Figura 4.1. 


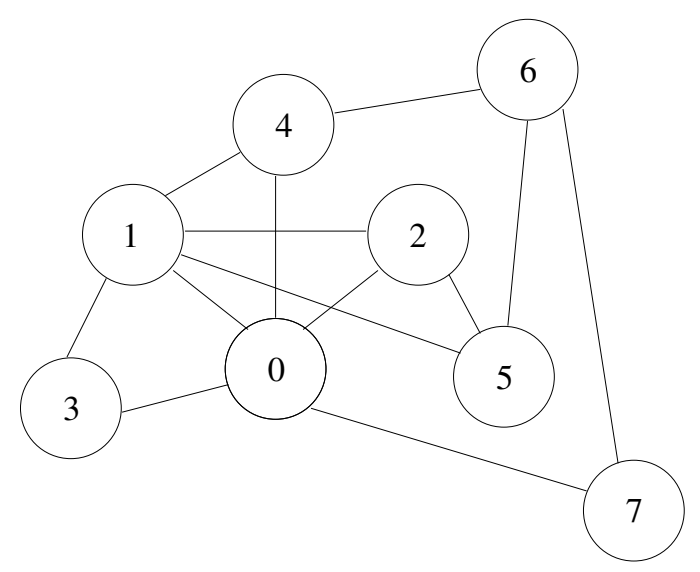

(a)

\begin{tabular}{|c|}
\hline Representação de Arestas \\
\hline$\{0,1\}$ \\
\hline$\{0,2\}$ \\
$\{1,2\}$ \\
\hline \\
$\{0,3\}$ \\
\hline$\{0,4\}$ \\
\hline$\{0,7\}$ \\
$\{1,3\}$ \\
$\{1,4\}$ \\
\hline$\{1,5\}$ \\
\hline$\{2,5\}$ \\
$\{4,6\}$ \\
\hline 55,6$\}$ \\
\hline$\{6,7\}$ \\
\hline
\end{tabular}

(b)

Figura 4.1: (a) Exemplo de uma Rede de Barabási-Albert com $N=8$ vértices e conectividade mínima $m=2$ arestas por vértice. (b) Representação de Arestas da rede desenhada em (a).

Na tabela 4.3 destacamos a representação de vizinhos e a distribuição de graus correspondentes a rede da Figura 4.1. 


\begin{tabular}{|c|c|c|}
\hline Vértice & Vizinhança & Grau \\
\hline 0 & $\{1,2,3,4,7\}$ & 5 \\
\hline 1 & $\{0,2,3,4,5\}$ & 5 \\
\hline 2 & $\{0,1,5\}$ & 3 \\
\hline 3 & $\{0,1\}$ & 2 \\
\hline 4 & $\{0,1,6\}$ & 3 \\
\hline 5 & $\{1,2,6\}$ & 3 \\
\hline 6 & $\{4,5,7\}$ & 3 \\
\hline 7 & $\{0,6\}$ & 2 \\
\hline
\end{tabular}

Tabela 4.3: Representação de vizinhos e distribuição de graus para a redeexemplo da Figura 4.1.

Perceba que, em relação ao sítio 0, temos que seus primeiros vizinhos são $\Gamma_{0}=\{1,2,3,4,7\}$, compatível com as linhas $2,3,6,8$ e 14 da Representação de Arestas na parte (b) da Figura 4.1.

É importante destacar que, para os cálculos pretendidos, a Representação de Vizinhos é bastante adequada, porém insuficiente, sendo necessário estendermos esta representação até a vizinhança de segunda ordem (segundos vizinhos) em virtude das interações do tipo Concordância entre Vizinhos de Segunda Espécie corresponderem a este alcance. Como ilustração, a Tabela 4.4 mostra a Representação de Segundos Vizinhos e a Distribuição para o Número de Segundos Vizinhos de nossa rede-exemplo. 


\begin{tabular}{|c|c|c|}
\hline Vértice & Vizinhança Segunda & Número de Segundos Vizinhos \\
\hline 0 & $\{5,6\}$ & 2 \\
\hline 1 & $\{6,7\}$ & 2 \\
\hline 2 & $\{3,4,6,7\}$ & 4 \\
\hline 3 & $\{2,4,5,7\}$ & 4 \\
\hline 4 & $\{2,3,5,7\}$ & 4 \\
\hline 5 & $\{0,3,4,7\}$ & 4 \\
\hline 6 & $\{0,1,2\}$ & 3 \\
\hline 7 & $\{1,2,3,4,5\}$ & 5 \\
\hline
\end{tabular}

Tabela 4.4: Representação de segundos vizinhos e distribuição para o número de segundos vizinhos para a rede-exemplo da Figura 4.1.

Para simples conferência, considere o sítio 0 e perceba que sua segunda vizinhaça é dada por $\left(\bigcup_{j} \Gamma_{j}, j \in \Gamma_{0}\right)-\left(\{0\} \cup \Gamma_{0}\right)=$ $=(\{0,2,3,4,5\} \cup\{0,1,5\} \cup\{0,1\} \cup\{0,1,6\} \cup\{0,6\})-(\{0\} \cup\{1,2,3,4,7\})$ $=\{5,6\}$, de acordo com a segunda linha da tabela.

Voltemos agora nossa atenção para para o cálculo do grau médio dos primeiros vizinhos de um sítio. A seguir, formalizamos o procedimento (algoritmo) empregado.

Cálculo do Grau Médio dos Primeiros Vizinhos:

1. Escolhido um sítio da rede, devemos tomar a média do grau de seus primeiros vizinhos. Podemos identificá-la como uma média parcial associada ao sítio.

2. Diante do valor médio parcial associado a cada sítio, tomamos a média destes valores sobre toda a rede. Reconhecemos este segundo valor médio como aquele associado, de fato, à rede.

3. Finalmente, calculamos a média dos valores associados a rede num ensemble satisfatório de simulações. 
No caso do sítio 0 de nossa rede-exemplo, sabemos que seus primeiros vizinhos são $\Gamma_{0}=\{1,2,3,4,7\}$, e que, $Q[1]=5, Q[2]=3, Q[3]=2$, $Q[4]=3, Q[7]=2$. Portanto, a conectividade média dos vizinhos de 0 é $k_{v}^{(0)}=3$. Os valores de $k_{v}^{(i)}$ para os demais sítios estão dispostos na Tabela 4.5.

\begin{tabular}{|c|c|}
\hline Vértice & Grau Médio dos Primeiros Vizinhos \\
\hline 0 & 3 \\
\hline 1 & 3,2 \\
\hline 2 & $4,333 \ldots$ \\
\hline 3 & 5 \\
\hline 4 & $4,333 \ldots$ \\
\hline 5 & $3,666 \ldots$ \\
\hline 6 & $2,666 \ldots$ \\
\hline 7 & 4 \\
\hline
\end{tabular}

Tabela 4.5: Distribuição de graus dos primeiros vizinhos para a rede-exemplo da Figura 4.1.

Concluímos, então, que o grau médio dos primeiros vizinhos associado a esta rede é $\left\langle k_{v}\right\rangle=3,775$.

Para o cálculo do fator de acoplamento médio, devemos seguir uma prescrição bastante semelhante a anterior, a menos de alguns complicadores relacionados ao número de conexões comuns entre os elementos da primeira e da segunda vizinhança de um sítio.

Cálculo do Fator de Acoplamento Médio entre as Vizinhanças:

1. Primeiramente, para cada segundo vizinho de um determinado sítio da rede, calculamos a fração de arestas compartilhadas entre aquele e um primeiro vizinho qualquer deste sítio.

2. Em seguida, tomamos a média destes valores sobre todos os segundos vizinhos do sítio considerado. Reconheceremos este resultado 
como o valor parcial do fator de acoplamento associado ao sítio.

3. Diante do fator de acoplamento parcial associado a cada sítio, tomamos a média destes valores sobre toda a rede.

4. Finalmente, calculamos a média dos valores associados a rede num ensemble satisfatório de simulações.

Vejamos agora, na prática, a etapas do cálculo para o sítio 0. A segunda vizinhança de 0 é $\Gamma_{0}^{(2)}=\{5,6\}$ e estes, por sua vez, se conectam aos sítios $A[5]=\{\underline{1}, \underline{2}, 6\}, A[6]=\{\underline{4}, 5, \underline{7}\}$, com os elementos sublinhados sendo pertencentes a primeira vizinhança de 0. Portanto, as frações de arestas compartilhadas entre a primeira vizinhança formam o conjunto $\left\{\frac{2}{3}, \frac{2}{3}\right\}$ e a média dos elementos deste conjunto é o fator médio de acoplamento parcial para o sítio 0 que vale $l_{0}=\frac{2}{3}=0,666 \ldots$. Este resultado indica que é bastante provável a ocorrência de interações do tipo Concordância entre Vizinhos de Segunda Espécie capaz de modificar a opinião do candidato 0 (há quase $70 \%$ de chances de ocorrer), como podemos constatar facilmente, pois, mais da metade dos elementos de cada conjunto acima estão sublinhados. A complementação dos cálculos de $\langle l\rangle$ para nossa rede-exemplo é apresentada nas tabelas 4.6 e 4.7. Fazemos um destaque para a segunda linha destas tabelas, onde podemos verificar os cálculos anteriores para $l_{0}$. 


\begin{tabular}{|c|c|}
\hline Vértice & Frações de Arestas Compartilhadas entre as Vizinhanças \\
\hline 0 & $\left\{\frac{2}{3}, \frac{2}{3}\right\}$ \\
\hline 1 & $\left\{\frac{2}{3}, \frac{1}{2}\right\}$ \\
\hline 2 & $\left\{1, \frac{2}{3}, \frac{1}{3}, \frac{1}{2}\right\}$ \\
\hline 3 & $\left\{\frac{2}{3}, \frac{2}{3}, \frac{1}{3}, \frac{1}{2}\right\}$ \\
\hline 4 & $\left\{\frac{2}{3}, 1, \frac{2}{3}, 1\right\}$ \\
\hline 5 & $\left\{\frac{2}{5}, \frac{1}{2}, \frac{2}{3}, \frac{1}{2}\right\}$ \\
\hline 6 & $\left\{\frac{2}{5}, \frac{2}{5}, \frac{1}{3}\right\}$ \\
\hline 7 & $\left\{\frac{1}{5}, \frac{1}{3}, \frac{1}{2}, \frac{2}{3}, \frac{1}{3}\right\}$ \\
\hline
\end{tabular}

Tabela 4.6: Frações de arestas compartilhadas entre os vértices da segunda e da primeira vizinhança para cada vértice de nossa rede-exemplo.

\begin{tabular}{|c|c|}
\hline Vértice & Fator Médio de Acoplamento Parcial \\
\hline 0 & $\frac{2}{3}$ \\
\hline 1 & $\frac{7}{12}$ \\
\hline 2 & $\frac{5}{8}$ \\
\hline 3 & $\frac{13}{24}$ \\
\hline 4 & $\frac{10}{12}$ \\
\hline 5 & $\frac{31}{60}$ \\
\hline 6 & $\frac{17}{45}$ \\
\hline 7 & $\frac{61}{150}$ \\
\hline
\end{tabular}

Tabela 4.7: Distribuição de fatores de acoplamento parcial para a redeexemplo da Figura 4.1.

O resultado $\langle l\rangle=0,56888 \ldots \approx 0,57$ para nossa rede-exemplo, representa um valor bastante alto para o fator de acoplamento. Nossa expectativa para redes BA é de que esse fator apresente valor muito inferior a unidade pois, em geral, numa rede de Barabási-Albert, são poucos os segundos vizinhos de um dado sítio que estão altamente conecta- 
dos com seus primeiros vizinhos, justificando as hipóteses assumidas por Vannucchi em sua dissertação de mestrado. Apesar disto, o simples abandono dos termos da equação relacionados a este parâmetro compromete fortemente a aproximação de campo médio, pois, o que é realmente relevante para os cálculos é o produto $\langle l\rangle\left\langle k^{\prime}\right\rangle$, que apresenta valor significativo. Desta forma conseguimos explicar as discrepâncias entre os resultados numéricos e analíticos de Vannucchi [6].

Na Tabela 4.8, apresentamos os valores tipicamente encontrados para os parâmetros médios de uma rede de Barabási-Albert com $\mathrm{N}=10000$ eleitores e conectividade mínima $\mathrm{m}=5$. Os valores determinados ao considerarmos um ensemble de 100 realizações da rede foram $\left\langle k_{v}\right\rangle=29,7 \pm 1,0,\langle k\rangle=9,997 \pm 0,001,\langle l\rangle=0,108 \pm 0,001$ e $\left\langle k^{\prime}\right\rangle=256,3 \pm 8,3$. De fato, o resultado para o fator de acoplamento entre as vizinhanças é muito menor do que a unidade, conforme esperado.

É razoável que $\left\langle k^{\prime}\right\rangle \gg\langle k\rangle$, pois, de acordo com a propriedade de conexão preferencial das redes de Barabási-Albert, é muito provável a existência de hubs junto a sítios de baixa conectividade. Além disso, é esperado que o grau médio dos vértices seja próximo do dobro da conectividade mínima m. 


\begin{tabular}{|c|c|c|c|c|}
\hline$\left\langle k_{v}\right\rangle$ & $\langle k\rangle$ & $\langle l\rangle$ & $\left\langle k^{\prime}\right\rangle$ & $w_{t}$ \\
\hline 29,757 & 9,997 & 0,108 & 257,657 & 0,261 \\
\hline 29,725 & 9,997 & 0,108 & 256,251 & 0,261 \\
\hline 28,281 & 9,997 & 0,108 & 244,930 & 0,264 \\
\hline 30,051 & 9,997 & 0,109 & 257,922 & 0,260 \\
\hline 30,115 & 9,997 & 0,108 & 261,256 & 0,260 \\
\hline 30,261 & 9,997 & 0,108 & 260,916 & 0,260 \\
\hline 31,372 & 9,997 & 0,108 & 269,719 & 0,258 \\
\hline 29,106 & 9,997 & 0,108 & 250,757 & 0,262 \\
\hline 29,199 & 9,997 & 0,108 & 252,295 & 0,262 \\
\hline 28,986 & 9,997 & 0,108 & 250,588 & 0,262 \\
\hline
\end{tabular}

Tabela 4.8: Amostra de resultados numéricos para os parâmetros médios de uma rede de Barabási-Albert com N=10.000 eleitores e conectividade mínima $\mathrm{m}=5$ entre eles. Consideramos um ensemble constituído de 100 simulações da rede.

Nas próximas seções, mostraremos que os cálculos de Vannucchi [6] são adequados apenas em redes do tipo grafo completo, onde as interações de segunda espécie nunca ocorrem.

\subsubsection{Análise Numérica de Outras Redes}

Essencialmente, para o tratamento numérico das redes regulares e do grafo completo, simplesmente modificamos o trecho do código fonte responsável pela construção do Ponteiro de Representação de Arestas ** $\mathrm{M}$ de acordo com a rede de interesse.

Esta etapa do trabalho é de significativa importância, pois, além de permitir a investigação das propriedades destas redes, representa uma forma bastante eficaz de colocar à prova o algoritmo, checando os resultados quanto a erros sistemáticos. 
Nas próximas tabelas, apresentaremos uma série típica de resultados referentes a uma rede quadrada com $\mathrm{N}=25$ eleitores, com o intuito de ilustrar os testes realizados ao algoritmo. À exceção do grafo completo, sempre consideraremos a hipótese adicional de condições periódicas de contorno na construção das redes. 


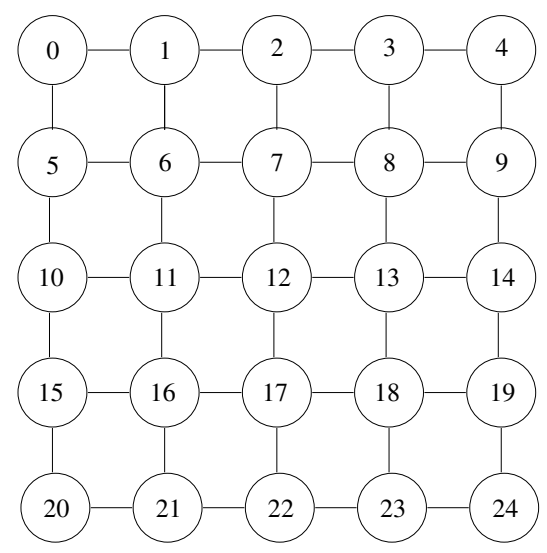

(a)

\begin{tabular}{|c|}
\hline Representação de Arestas \\
\hline$\{0,1\}$ \\
\hline$\{0,5\}$ \\
\hline$\{0,4\}$ \\
\hline$\{0,20\}$ \\
$\{1,2\}$ \\
$\{1,6\}$ \\
\hline$\{1,21\}$ \\
$\{2,3\}$ \\
$\{2,7\}$ \\
$\{2,22\}$ \\
$\{3,4\}$ \\
$\{3,8\}$ \\
$\{3,23\}$ \\
$\ldots$ \\
\hline
\end{tabular}

(b)

Figura 4.2: (a) Exemplo de rede quadrada com $N=25$ eleitores. (b) Representação de Arestas referente aos quatro primeiros elementos da rede em (a).

Uma clara manifestação das condições periódicas de contorno está indicada na Tabela 4.9, onde todos os sítios, até mesmo os sítios da borda da rede, apresentam quatro vizinhos. 


\begin{tabular}{|c|c|c|}
\hline Vértice & Vizinhos & Grau \\
\hline 0 & $\{1,4,5,20\}$ & 4 \\
\hline 1 & $\{0,2,6,21\}$ & 4 \\
\hline 2 & $\{1,3,7,22\}$ & 4 \\
\hline 3 & $\{2,4,8,23\}$ & 4 \\
\hline 4 & $\{0,3,9,24\}$ & 4 \\
\hline 5 & $\{0,6,9,10\}$ & 4 \\
\hline 6 & $\{1,5,7,11\}$ & 4 \\
\hline 7 & $\{2,6,8,12\}$ & 4 \\
\hline 8 & $\{3,7,9,13\}$ & 4 \\
\hline 9 & $\{4,5,8,14\}$ & 4 \\
\hline 10 & $\{5,11,14,15$ & 4 \\
\hline 11 & $\{6,10,12,16\}$ & 4 \\
\hline 12 & $\{7,11,13,17\}$ & 4 \\
\hline 13 & $\{8,12,14,18\}$ & 4 \\
\hline 14 & $\{9,10,13,19\}$ & 4 \\
\hline 15 & $\{10,16,19,20\}$ & 4 \\
\hline 16 & $\{11,15,17,21\}$ & 4 \\
\hline 17 & $\{12,16,18,22\}$ & 4 \\
\hline 18 & $\{13,17,19,23\}$ & 4 \\
\hline 19 & $\{14,15,18,24\}$ & 4 \\
\hline 20 & $\{0,15,21,24\}$ & 4 \\
\hline 21 & $\{1,16,20,22\}$ & 4 \\
\hline 22 & $\{2,17,21,23\}$ & 4 \\
\hline 23 & $\{3,18,22,24\}$ & 4 \\
\hline 24 & $\{4,19,20,23\}$ & 4 \\
\hline
\end{tabular}

Tabela 4.9: Representação de vizinhos e distribuição de graus para a rede quadrada representada na Figura 4.2.

Na Tabela 4.10, todos os sítios apresentam oito segundos vizinhos, 
como esperado.

\begin{tabular}{|c|c|}
\hline Vértice & Número de Segundos Vizinhos \\
\hline 0 & 8 \\
\hline 1 & 8 \\
\hline 2 & 8 \\
\hline 3 & 8 \\
\hline 4 & 8 \\
\hline 5 & 8 \\
\hline 6 & 8 \\
\hline 7 & 8 \\
\hline 8 & 8 \\
\hline 9 & 8 \\
\hline 10 & 8 \\
\hline 11 & 8 \\
\hline 12 & 8 \\
\hline 13 & 8 \\
\hline 14 & 8 \\
\hline 15 & 8 \\
\hline 16 & 8 \\
\hline 17 & 8 \\
\hline 18 & 8 \\
\hline 19 & 8 \\
\hline 20 & 8 \\
\hline 21 & 8 \\
\hline 22 & 8 \\
\hline 23 & 8 \\
\hline 24 & 8 \\
\hline
\end{tabular}

Tabela 4.10: Número de segundos vizinhos para os vértices da rede quadrada apresentada na Figura 4.2. 
A Tabela 4.11 apresenta os resultados numéricos dos parâmetros médios para a rede quadrada em questão. Os resultados estão de acordo com aqueles estabelecidos no capítulo anterior.

\begin{tabular}{|c|c|c|c|c|}
\hline$\left\langle k_{v}\right\rangle$ & $\langle k\rangle$ & $\langle l\rangle$ & $\left\langle k^{\prime}\right\rangle$ & $w_{t}$ \\
\hline 4 & 4 & 0,375 & 8 & 0,327 \\
\hline
\end{tabular}

Tabela 4.11: Resultados numéricos dos parâmetros médios para a rede quadrada da Figura 4.2.

Vejamos agora, na Tabela 4.12, a representação de vizinhos e a distribuição de graus para um exemplo de grafo completo bastante simplificado com $\mathrm{N}=10$ candidatos. Diferentemente das redes regulares e das expectativas geradas no capítulo 3, o tratamento numérico do grafo completo apresentou peculiaridades quanto à segunda vizinhança e requer atenção especial.

\begin{tabular}{|c|c|c|}
\hline Vértice & Vizinhança & Grau \\
\hline 0 & $\{1,2,3,4,5,6,7,8,9\}$ & 9 \\
\hline 1 & $\{0,2,3,4,5,6,7,8,9\}$ & 9 \\
\hline 2 & $\{0,1,3,4,5,6,7,8,9\}$ & 9 \\
\hline 3 & $\{0,1,2,4,5,6,7,8,9\}$ & 9 \\
\hline 4 & $\{0,1,2,3,5,6,7,8,9\}$ & 9 \\
\hline 5 & $\{0,1,2,3,4,6,7,8,9\}$ & 9 \\
\hline 6 & $\{0,1,2,3,4,5,7,8,9\}$ & 9 \\
\hline 7 & $\{0,1,2,3,4,5,6,8,9\}$ & 9 \\
\hline 8 & $\{0,1,2,3,4,5,6,7,9\}$ & 9 \\
\hline 9 & $\{0,1,2,3,4,5,6,7,8\}$ & 9 \\
\hline
\end{tabular}

Tabela 4.12: Representação de vizinhos e distribuição de graus para um grafo completo com $\mathrm{N}=10$ candidatos.

Perceba que um dado sítio mantém conexões com todos os outros 
sítios da rede, e, consequentemente, o grau de cada sítio é uma constante e igual a 9 .

Segundo os cálculos do capítulo anterior, o grafo completo apresenta $\langle k\rangle=\left\langle k_{v}\right\rangle=\left\langle k^{\prime}\right\rangle$, entretanto, esta propriedade deve ser revisada. A rigor, ao aplicarmos a definição de segunda vizinhança obtemos o conjunto vazio como resultado, ou seja, $\gamma_{i}=\varnothing, \forall i$.

De fato, os sítios não apresentam segunda vizinhança, pois, a primeira vizinhança já abrange toda a rede, implicando necessariamente em $\langle k\rangle=\left\langle k_{v}\right\rangle=N-1$ e $\langle l\rangle=\left\langle k^{\prime}\right\rangle=0$. Os resultados numéricos chamam a atenção para este fato, como mostra a Tabela 4.13.

\begin{tabular}{|c|c|c|}
\hline Vértice & Segundos Vizinhos & Número de Segundos Vizinhos \\
\hline 0 & -1 & 0 \\
\hline 1 & -1 & 0 \\
\hline 2 & -1 & 0 \\
\hline 3 & -1 & 0 \\
\hline 4 & -1 & 0 \\
\hline 5 & -1 & 0 \\
\hline 6 & -1 & 0 \\
\hline 7 & -1 & 0 \\
\hline 8 & -1 & 0 \\
\hline 9 & -1 & 0 \\
\hline
\end{tabular}

Tabela 4.13: Representação de segundos vizinhos para um grafo completo com $\mathrm{N}=10$ eleitores. O algoritmo retornava -1 na inexistência de segundos vizinhos.

Esta divergência entre os resultados não é completamente inaceitável e pode ser tolerada se adotarmos uma definição mais flexível para a segunda vizinhança, a saber, $\tilde{\gamma}_{i}=\bigcup_{j} \Gamma_{j}-\{i\} ; j \in \Gamma_{i}$. Nestas circunstâncias, a primeira vizinhança coincide, de fato, com a segunda 
vizinhança e a igualdade entre as conectividades é recuperada.

A ausência de segunda vizinhança por parte dos sítios de um grafo completo exige que a equação mestra seja reescrita devido a impossibilidade de transições de segunda espécie. Vejamos a equação para este caso.

$$
\begin{aligned}
\Delta \eta^{0}= & -(1-w) \frac{\langle k\rangle}{\left\langle k_{v}\right\rangle} \eta^{0}\left(1-\eta^{0}\right)\left[\frac{1}{\left\langle k_{v}\right\rangle}+\left(1-\eta^{0}\right)\right]-w \eta^{0} \\
\Delta \eta^{\sigma}= & (1-w) \frac{\langle k\rangle}{\left\langle k_{v}\right\rangle} \eta^{\sigma}\left[\frac{1}{\left\langle k_{v}\right\rangle} \eta^{0}+\left(1-\eta^{\sigma}\right) \eta^{\sigma}-\frac{\left(1-\eta^{0}-\eta^{\sigma}\right)^{2}}{\left(N_{c}-1\right)}\right]+ \\
& +w\left[\left(1-\eta^{\sigma}\right) \frac{1}{N_{c}}-\eta^{\sigma}\left(1-\frac{1}{N_{c}}\right)\right]
\end{aligned}
$$

As soluções estacionárias são facilmente obtidas ao tomarmos $\langle l\rangle=$ 0 nas soluções do capítulo 3.

$$
\begin{aligned}
& \eta^{0}=0 \\
& \eta_{0}^{\sigma}=\frac{1}{N_{c}} \\
& \eta_{ \pm}^{\sigma}=\frac{1}{2} \pm \sqrt{\frac{1}{4}-\frac{w}{(1-w)} \frac{\left\langle k_{v}\right\rangle}{\langle k\rangle} \frac{\left(N_{c}-1\right)}{N_{c}}} \\
& 0 \leq w<\tilde{w} \Rightarrow \eta_{-}^{\sigma}<\eta_{0}^{\sigma}<\eta_{+}^{\sigma} \text {, com } \eta_{0}^{\sigma} \text { estável } \\
& \tilde{w}<w<w_{t} \Rightarrow \eta_{0}^{\sigma}<\eta_{-}^{\sigma}<\eta_{+}^{\sigma} \text {, com } \eta_{-}^{\sigma} \text { estável } \\
& w>w_{t} \quad \Rightarrow \text { apenas } \eta_{0}^{\sigma} \text { é estacionária (e estável) } \\
& \tilde{w} \equiv\left[1+\frac{\left\langle k_{v}\right\rangle}{\langle k\rangle} N_{c}\right]^{-1} \text { e } w_{t} \equiv\left[1+4 \frac{\left\langle k_{v}\right\rangle}{\langle k\rangle} \frac{\left(N_{c}-1\right)}{N_{c}}\right]^{-1}
\end{aligned}
$$

No caso particular $w=0$ (sem ruído), a solução estacionária continua representando um estado de consenso, como esperado.

O diagrama de bifurcação e o parâmetro de ordem continuam apresentando o mesmo comportamento típico mostrado nas Figuras 3.6 e 3.7 . 
A Tabela 4.14 apresenta os valores dos pontos críticos para o grafo completo.

\begin{tabular}{|c|c|c|c|}
\hline Rede & - & $\tilde{w}$ & $w_{t}$ \\
\hline \multirow{4}{*}{$\tilde{\gamma}$} & $N_{c}=2$ & $1 / 2$ & $1 / 2$ \\
\cline { 2 - 4 } & $N_{c}=10$ & $1 / 6$ & $5 / 14$ \\
\cline { 2 - 4 } & $N_{c}=\infty$ & 0 & $1 / 3$ \\
\hline \multirow{4}{*}{$\gamma$} & $N_{c}=2$ & $1 / 3$ & $1 / 3$ \\
\cline { 2 - 4 } & $N_{c}=10$ & $1 / 11$ & $5 / 23$ \\
\cline { 2 - 4 } & $N_{c}=\infty$ & 0 & $1 / 5$ \\
\hline
\end{tabular}

Tabela 4.14: Parâmetros críticos para o Modelo Sznajd Complexo numa aproximação de campo médio considerando as vizinhanças $\gamma$ e $\tilde{\gamma}$ de um grafo completo.

Certamente os resultados que descrevem mais exatamente a dinâmica do modelo são aqueles que utilizam a definição $\gamma$ para a segunda vizinhança, entretanto, os resultados que utilizam $\tilde{\gamma}$ oferecem uma visão alternativa para o caso de redes regulares no limite de altas dimensionalidades, conforme discussões realizadas no capítulo 3. A equivalência entre os dois casos que estabelecemos no capítulo anterior não é completa e justificamos este fato pelo diferenciado comportamento assintótico de $\langle l\rangle\left\langle k^{\prime}\right\rangle$ apresentado por eles.

Os resultados da versão revisada concordam com os cálculos realizados por Vannucchi em sua tese [6], mostrando que aquele tratamento, apesar de limitado, é capaz de descrever bem o Modelo Sznajd Complexo em primeira aproximação. 


\subsection{Resultados Numéricos para o Modelo sem Ru- ído}

Nesta seção, apresentaremos os principais resultados numéricos para a dinâmica do Modelo Sznajd Complexo na ausência de ruído. Como principal objetivo verificaremos que o modelo sempre apresenta o consenso como único estado estado estacionário para as diferentes redes abordadas.

Em geral o estado estacionário é atingido a partir de uma quantidade relativamente pequena de Tempos de Monte Carlo, caracterizando o tempo de relaxação $\tau$ da dinâmica. O comportamento transiente é marcado por grandes flutuações no número de votos dos candidatos.

Voltaremos nossa atenção, principalmente, aos resultados realizados numa rede de Barabási-Albert, entretanto, simulações envolvendo redes regulares serão apresentadas e receberão devida atenção. Resultados preliminares obtidos por minha orientadora e seu aluno de Iniciação Científica César Uliana sugerem que estas redes também são capazes de reproduzir as leis de escala observadas desde que a dimensionalidade da rede seja suficientemente alta.

A seguir, apresentaremos uma grande quantidade de simulações mostrando evoluções típicas do número de votos por candidato.

\subsubsection{Séries Temporais na Rede de Barabási-Albert}

Na figura 4.3 apresentamos o comportamento típico de séries temporais para o Modelo Sznajd Complexo com $N_{c}=10$ candidatos numa rede de Barabási-Albert de $N=1000$ eleitores e conectividade mínima $\mathrm{m}=5$. 

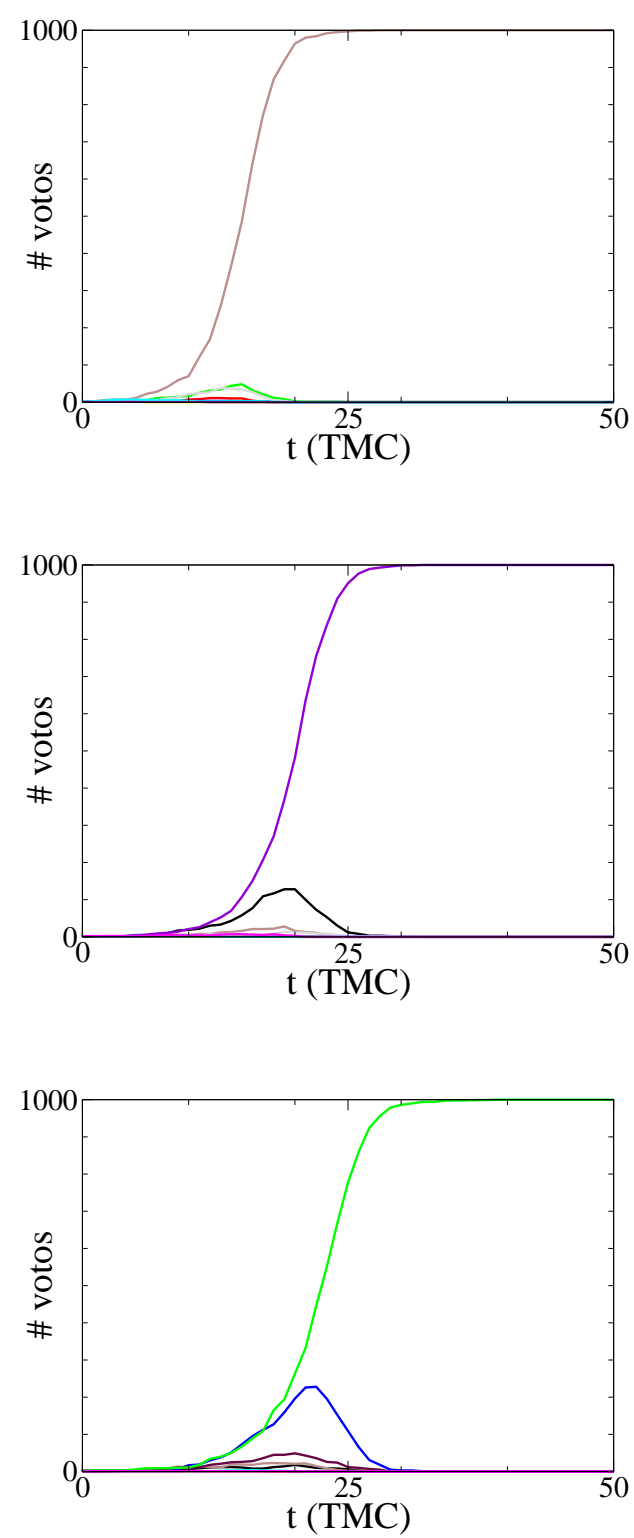

Figura 4.3: Evolução temporal da distribuição de votos para o Modelo Sznajd Complexo sem Ruído com $N_{c}=10$ candidatos numa rede de BarabásiAlbert com $N=1000$ eleitores e conectividade mínima $m=5$. 
O estado de consenso é sempre alcançado com tempos de relaxação tipicamente da ordem de $\tau=30 \mathrm{TMC}\left(\frac{\tau}{N} \ll 1 \mathrm{TMC}\right)$. A lei de escala pode ser observada em torno de $t=20 \mathrm{TMC}$.

O caso particular em que temos $N_{c}=2$ candidatos é destacado na figura 4.4 e apresenta comportamento qualitativo semelhante ao anterior com tempos de relaxação da ordem de $\tau=20$ TMC. É esperado um tempo de relaxação menor neste caso por representar uma situação de menor competitividade entre os candidatos. 

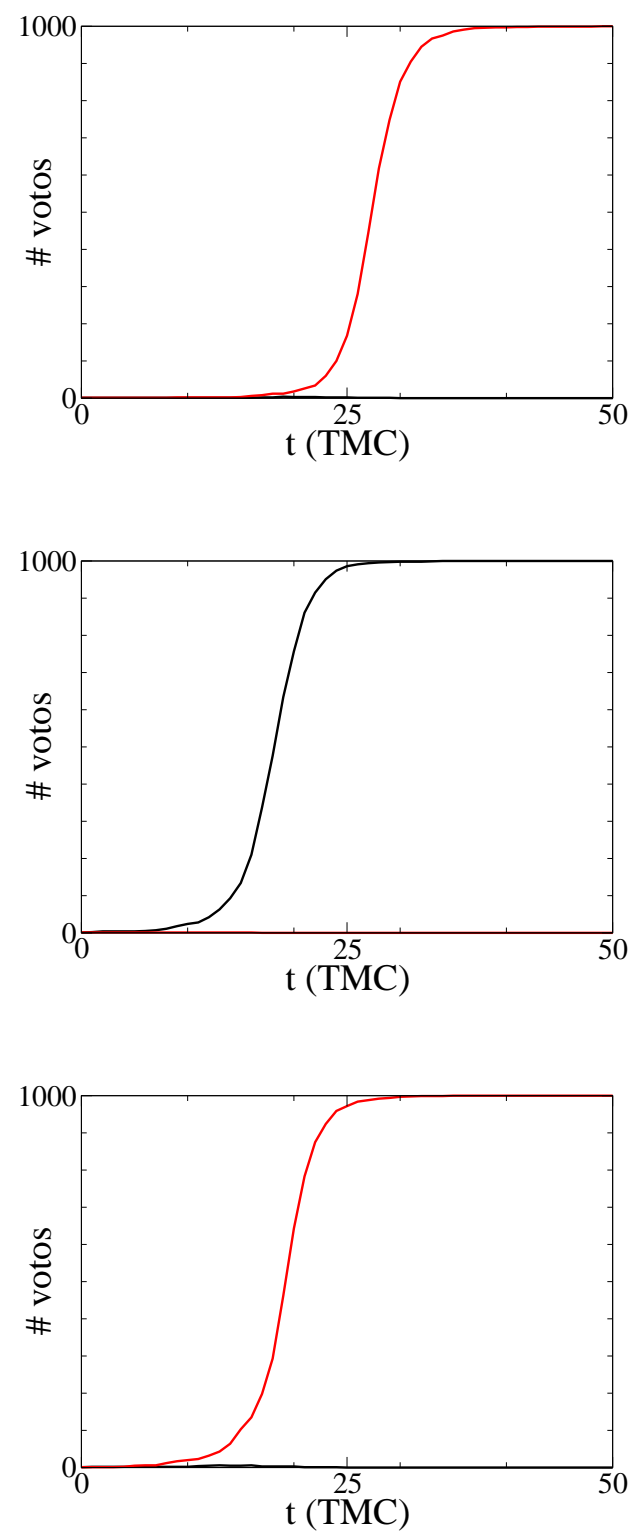

Figura 4.4: Evolução temporal da distribuição de votos para o Modelo Sznajd Complexo sem Ruído com $N_{c}=2$ candidatos numa rede de BarabásiAlbert com $N=1000$ eleitores e conectividade mínima $m=5$. 
Em geral não se observam mudanças significativas no comportamento qualitativo destas curvas em função do número de candidatos ou de propriedades da rede.

\subsubsection{Séries Temporais para Redes Regulares}

No caso de redes regulares, o comportamento das séries temporais é fortemente influenciado pela dimensionalidade da rede. $\mathrm{O}$ anel de eleitores apresenta um elevado tempo de relaxação, da ordem de $\tau \sim$ $10^{5}$ TMC, além de flutuações muito mais intensas e prologadas em seu estado transiente do que a rede de Barabási-Albert. A duração prolongada das flutuações é responsável pelo aspecto pouco suavizado das curvas, afetando o estado estacionário que pode ser alcançado de maneira bastante súbita.

A Figura 4.5 mostra séries temporais típicas para o Modelo Sznajd Complexo num anel de $N=1000$ eleitores e $N_{c}=10$ candidatos. 

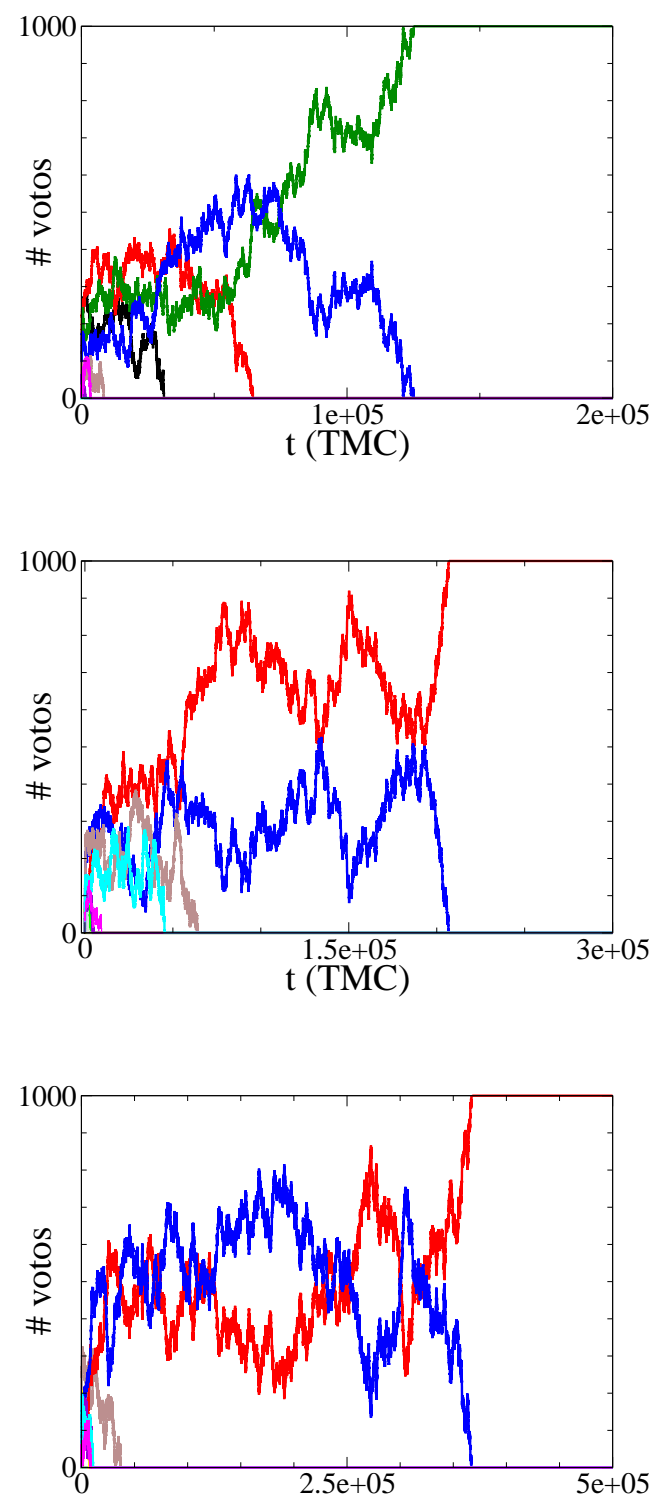

Figura 4.5: Evolução temporal da distribuição de votos para o Modelo Sznajd Complexo sem Ruído com $N_{c}=10$ candidatos num anel de $N=1000$ eleitores. 
Na Figura 4.6 destacamos a mesma situação para o caso particular de $N_{c}=2$ candidatos.

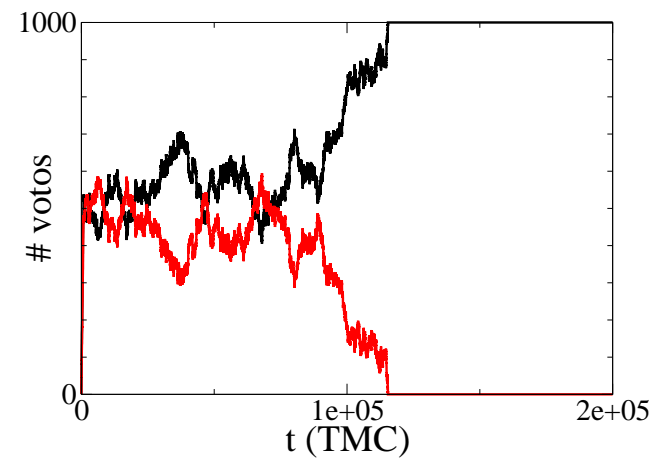

Figura 4.6: Evolução temporal da distribuição de votos para o Modelo Sznajd Complexo sem Ruído com $N_{c}=2$ candidatos num anel de $N=1000$ eleitores.

A seguir, na Figura 4.7, apresentamos séries temporais para o modelo numa rede quadrada com $N=32^{2}=1024$ eleitores e $N_{c}=10$ candidatos. Redes quadradas apresentam tempo de relaxação muito menores do que o anel de eleitores, mas continuam sendo bem maiores do que os tempos na rede de Barabási-Albert, com valores em torno de $\tau \sim 10^{3} \mathrm{TMC}\left(\tau_{1 D} / \tau_{2 D} \sim 10^{2}\right)$. Nos demais aspectos, o comportamento destes dois casos apresentam muitas semelhanças. 

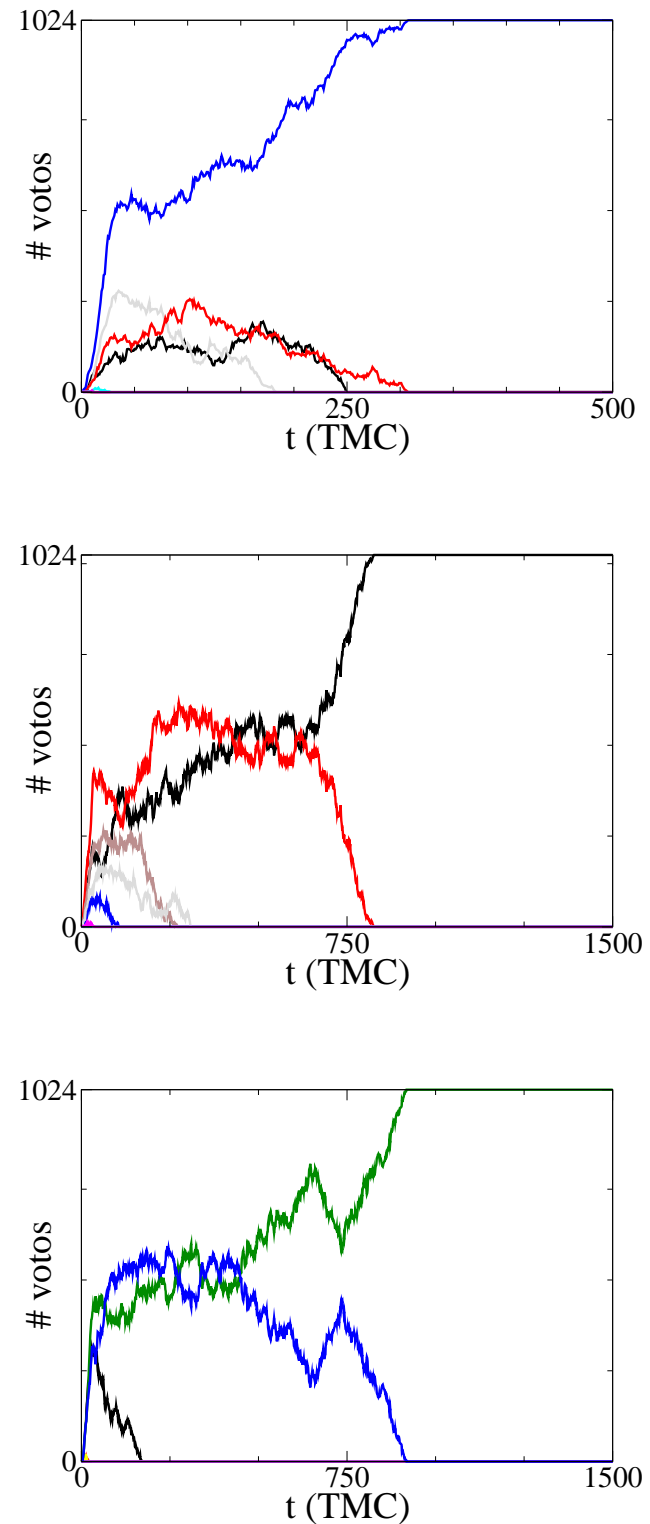

Figura 4.7: Evolução temporal da distribuição de votos para o Modelo Sznajd Complexo sem Ruído numa rede quadrada com $N=1024$ eleitores e $N_{c}=10$ candidatos. 
Na Figura 4.8 consideramos a mesma situação para o caso de $N_{c}=2$ candidatos.

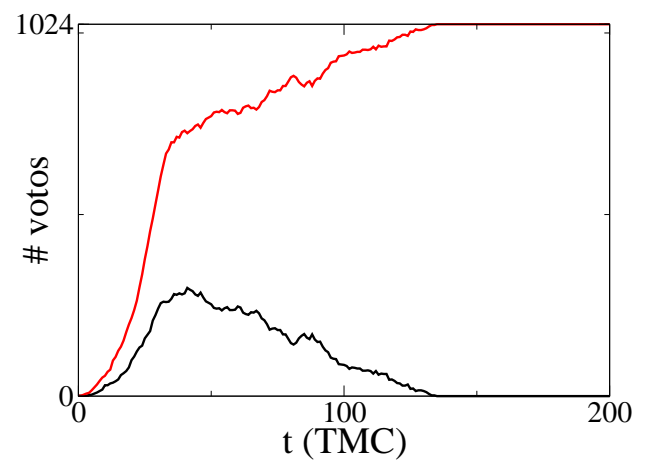

Figura 4.8: Evolução temporal da distribuição de votos para o Modelo Sznajd Complexo sem Ruído numa rede quadrada com $N=1024$ eleitores e $N_{c}=2$ candidatos.

Perceba que o tempo de relaxação para dois candidatos situa-se em torno de $\tau \sim 10^{2} \mathrm{TMC}$, indicando considerável sensibilidade em relação ao número de candidatos. É de se esperar que o tempo de relaxação diminua na medida em que o número de candidatos diminue, assim como apresentado pela rede BA. O caso unidimensional não apresenta diferenças acentuadas neste aspecto e implica em tempos de relaxação praticamente independentes do número de candidatos. A topologia unidimensional favorece o aparecimento de facções muito resistentes a opiniões que divergem da sua e a competição entre elas determina os altos índices no tempo de relaxação deste caso.

Em seguida, apresentamos séries temporais típicas para o Modelo Sznajd Complexo em redes cúbicas com $N=10^{3}=1000$ eleitores e $N_{c}=10$ candidatos através da Figura 4.9 . 

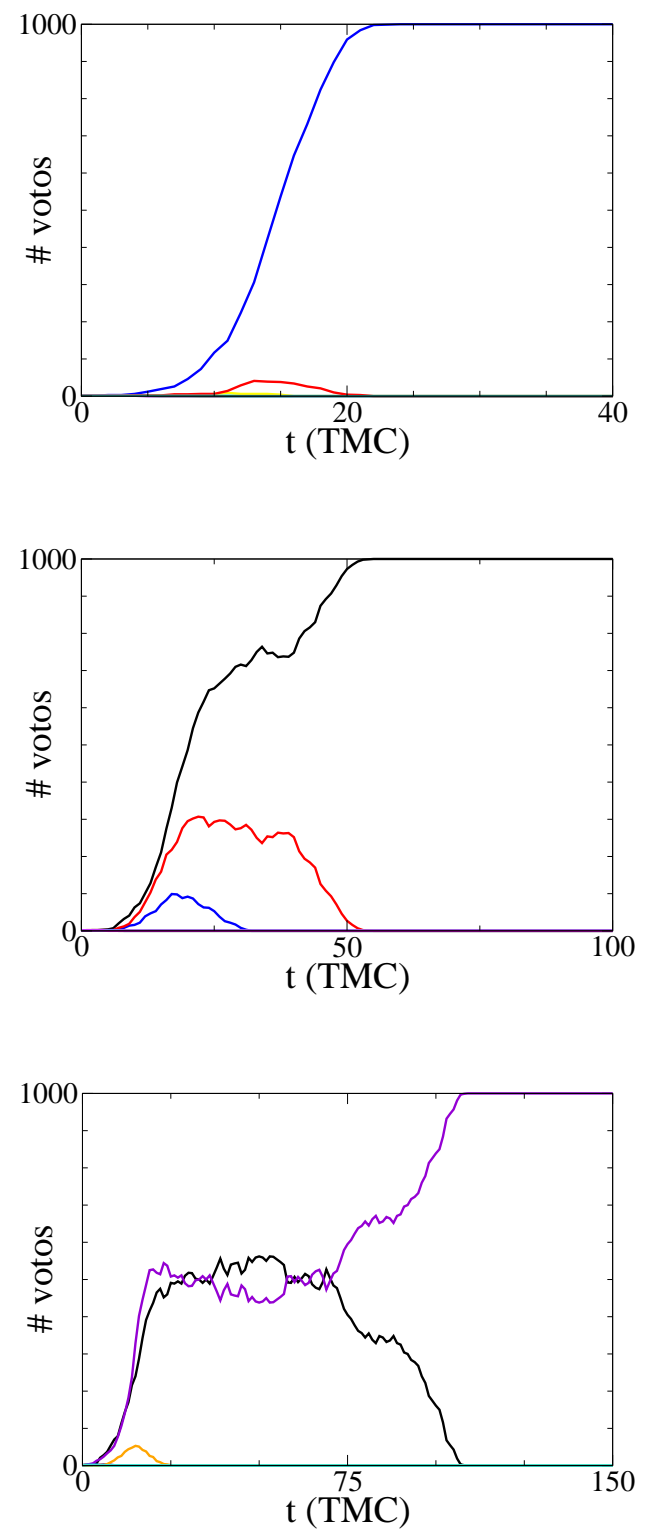

Figura 4.9: Evolução temporal da distribuição de votos para o Modelo Sznajd Complexo sem Ruído com $N_{c}=10$ candidatos numa rede cúbica com $N=1000$ eleitores. 
Na Figura 4.10 temos a mesma situação para o caso de $N_{c}=2$ candidatos.

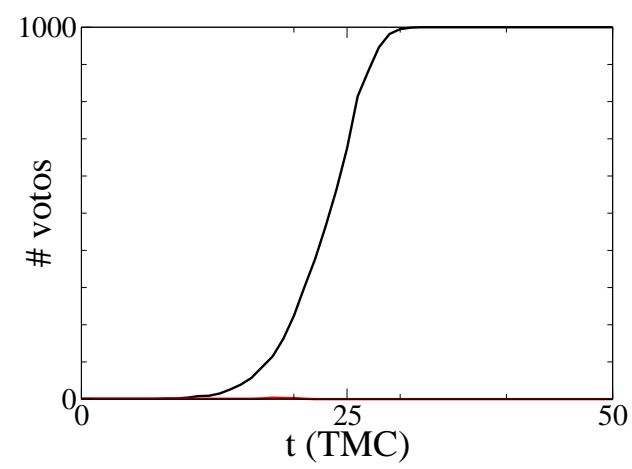

Figura 4.10: Evolução temporal da distribuição de votos para o Modelo Sznajd Complexo sem Ruído com $N_{c}=2$ candidatos numa rede cúbica com $N=1000$ eleitores.

Perceba que o tempo de relaxação típico é da ordem de $\tau_{3 D} \sim 10^{2}$ TMC, bem menor do que as redes quadradas, indicando a sensibilidade de $\tau$ em relação à dimensionalidade da rede.

Na subseção seguinte, detalharemos a análise do modelo em grafos completos.

\subsubsection{Séries Temporais em Grafos Completos}

Na Figura 4.11 apresentamos séries temporais típicas para o Modelo Sznajd Complexo em grafos completos com $N=10^{3}=1000$ eleitores e $N_{c}=10$ candidatos. 

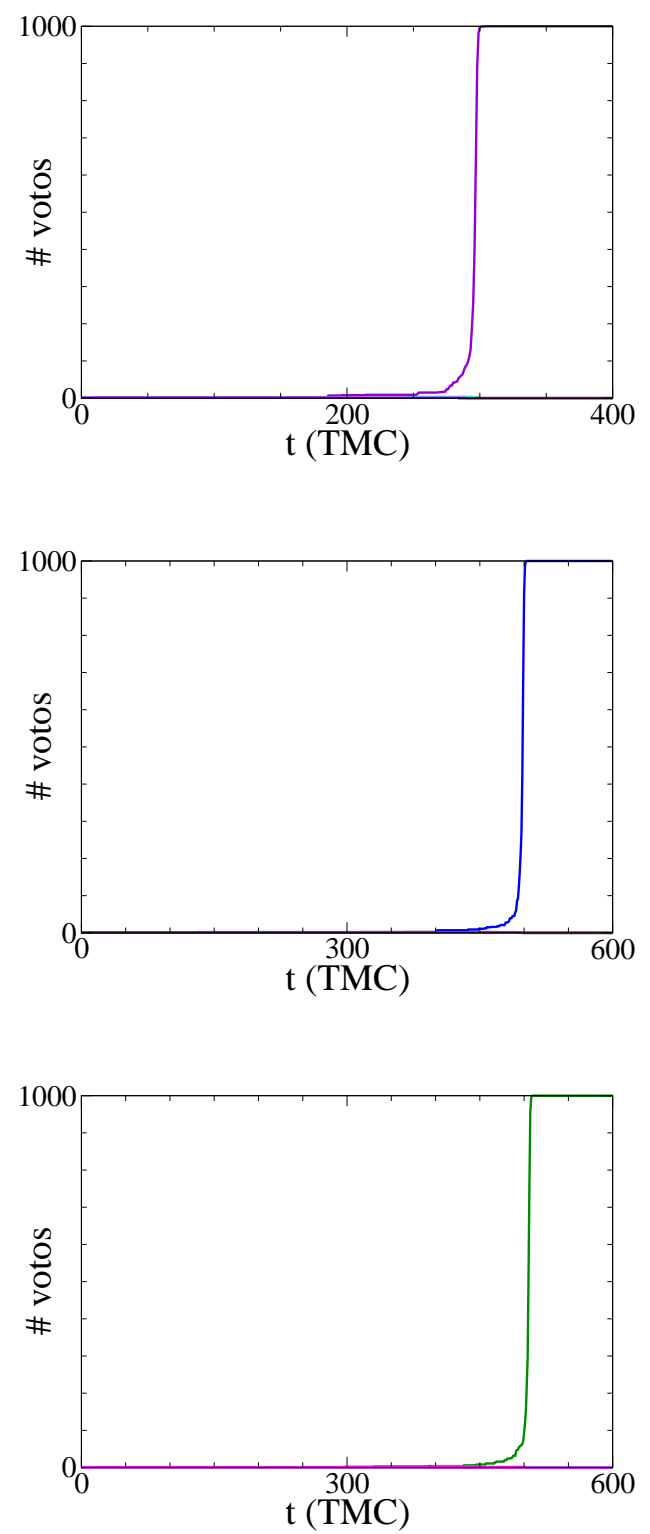

Figura 4.11: Evolução temporal da distribuição de votos para o Modelo Sznajd Complexo sem Ruído com $N_{c}=10$ candidatos num grafo completo com $N=1000$ eleitores. 
O tempo de relaxação da dinâmica nestas redes é tipicamente da ordem de $\tau \sim 500$ TMC, apresentando transiente bastante atípico, com a ausência de flutuações na quantidade de votos de cada candidato. O processo fica estagnado com predominância de indecisos pela rede durante todo o transiente.

Observa-se, também, que a dinâmica do modelo é insensível a variações no número de candidatos, de acordo com a Figura 4.12, que mostra uma série temporal típica para o caso de $N_{c}=2$ candidatos.

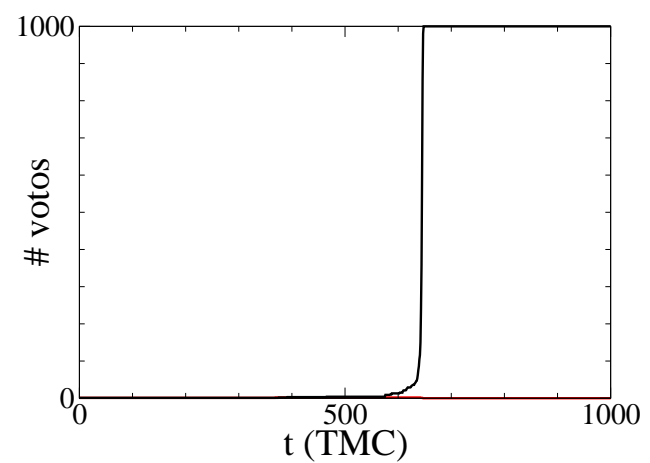

Figura 4.12: Evolução temporal da distribuição de votos para o Modelo Sznajd Complexo sem Ruído com $N_{c}=2$ candidatos num grafo completo com $N=1000$ eleitores.

Os resultados também indicam que as redes regulares, no limite de altas dimensionalidades, devem apresentar um estado estacionário de consenso de acordo com discussões realizadas no capítulo 3.

\subsubsection{Comportamento dos Indecisos}

É interessante explorarmos as séries temporais para o número de indecisos nas diferentes redes estudadas nesta seção, como mostra a Figura 4.13. 


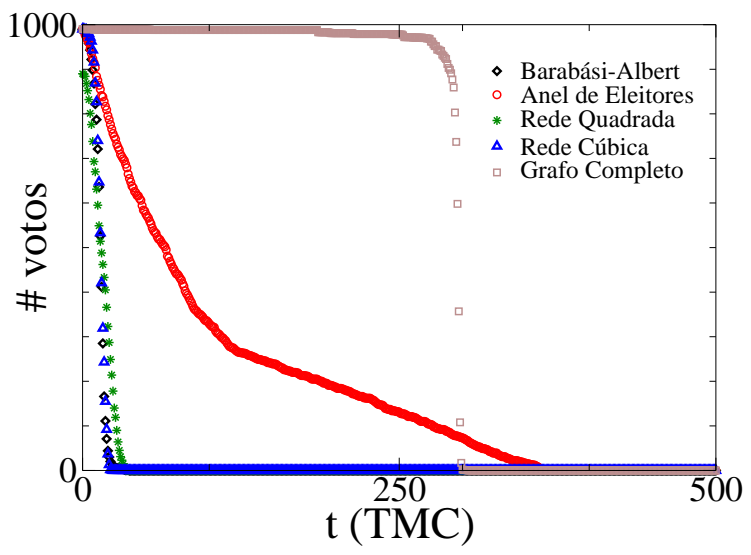

Figura 4.13: Comportamento dos indecisos nas diferentes redes estudadas. As redes utilizadas obedeciam um padrão com $N=1000$ eleitores e $N_{c}=2$ candidatos.

Como esperado, as séries temporais mostram um decaimento no número de indecisos, que sempre tende a zero, durante um intervalo de tempo que denominaremos tempo de relaxação dos indecisos, $\tau_{0}$. Esperamos, também, que o tempo de relaxação dos indecisos seja da ordem do tempo de relaxação da dinâmica (ou, pelo menos, um bom estimante inferior). Em grafos completos, esta expectativa é, de fato, verdadeira, devido às propriedades peculiares de seu transiente.

Observa-se que o anel de eleitores é a rede que apresenta o maior índice para o tempo de relaxação, redes quadradas e grafos completos assumem valores intermediários, enquanto que redes cúbicas e redes de Barabási-Albert apresentam índices muito inferiores ao do anel de eleitores. Podemos concluir que $\tau_{B A} \sim \tau_{3 D}<\tau_{K_{N}} \sim \tau_{2 D} \ll \tau_{1 D}$.

Na Tabela 4.15 resumimos os principais resultados desta seção ao apresentar os tempos de relaxação de todas as redes estudadas. Os 
valores sobrescritos representam o número de candidatos considerados em cada rede, por exemplo, $\tau^{(2)}$ significa o tempo de relaxação para uma rede com $N_{c}=2$ candidatos.

\begin{tabular}{|c|c|c|c|}
\hline Rede & $\tau^{(10)}(\mathrm{TMC})$ & $\tau^{(2)}(\mathrm{TMC})$ & $\tau_{0}^{(10)}(\mathrm{TMC})$ \\
\hline Anel de Eleitores & $3.10^{5}$ & $1.10^{5}$ & 400 \\
\hline Rede Quadrada & $1.10^{3}$ & $1.10^{2}$ & 40 \\
\hline Grafo Completo & 500 & 500 & 300 \\
\hline Rede Cúbica & 100 & 30 & 30 \\
\hline Barabási-Albert & 30 & 20 & 30 \\
\hline
\end{tabular}

Tabela 4.15: Tempos de relaxação em diferentes redes para o Modelo Sznajd Complexo sem Ruído. O índice sobrescrito refere-se ao número de candidatos considerados na rede e o índice 0 subescrito refere-se ao tempo de relaxação dos indecisos.

\subsection{Resultados Numéricos para o Modelo com Ru- ído}

Nesta seção mostraremos a existência de uma transição de fases entre a fase de vitória isolada e a fase ruidosa, através de cálculos numéricos. Com estes cálculos, determinaremos o diagrama de bifurcação e o parâmetro de ordem e os confrontaremos com resultados analíticos de campo médio.

\subsubsection{Séries Temporais na Rede de Barabási-Albert}

Através da Figura 4.14 procuramos evidenciar, sob o ponto de vista numérico, a principal consequência da inclusão do ruído à dinâmica, isto é, o desaparecimento do estado estacionário de consenso para valores suficientemente elevados de ruído. Nas séries temporais repre- 
sentadas nesta figura, a intensidade do ruído é suficientemente baixa $\left(w<w_{t}\right)$ para permitir um estado estacionário com a maioria do eleitorado tendo a mesma opinião, caracterizando a fase ordenada do sistema.

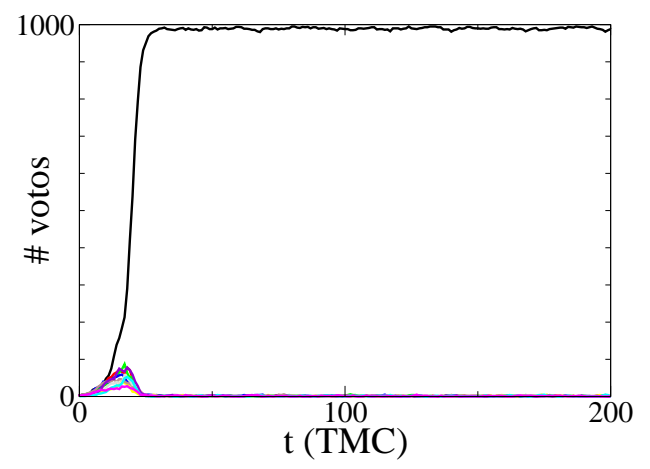

(a)

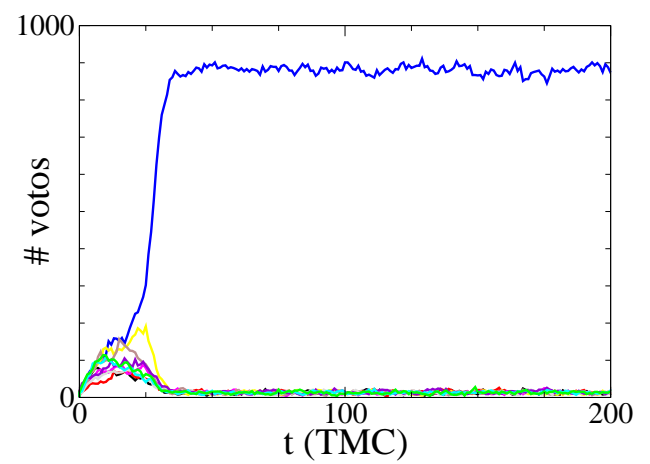

(b)

Figura 4.14: Evolução temporal da distribuição de votos para o Modelo Sznajd Complexo com Ruído numa Rede de Barabási-Albert com $N=1000$ eleitores e $N_{c}=10$ candidatos. (a) $w=0,01$ e (b) $w=0,10$.

Para intensidades de ruídos suficientemente altas $\left(w>w_{t}\right)$, o comportamento das séries temporais se afasta tanto da situação de con- 
senso que o estado estacionário passa a ser representado por um empate técnico entre todos os candidatos, caracterizando a fase desordenada do sistema, como mostra a Figura 4.15.

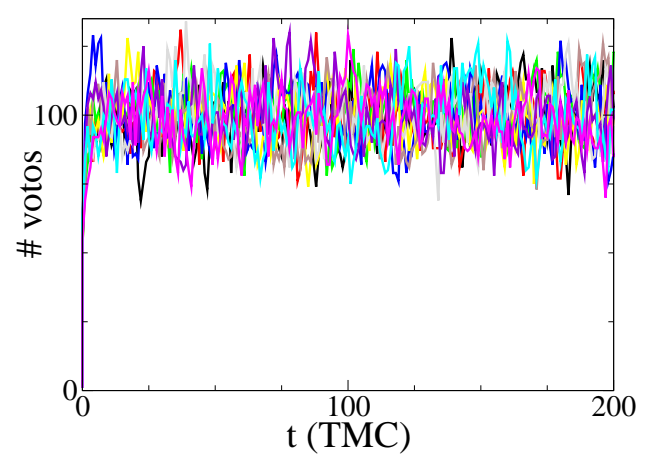

(a)

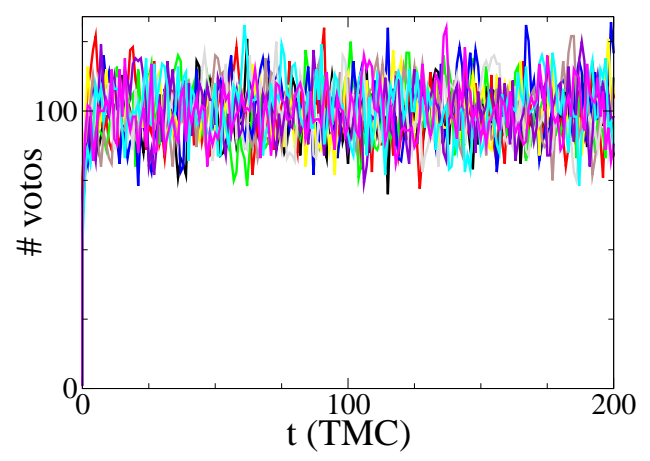

(b)

Figura 4.15: Evolução temporal da distribuição de votos para o Modelo Sznajd Complexo com Ruído numa Rede de Barabási-Albert com $N=1000$ eleitores e $N_{c}=10$ candidatos. (a) $w=0,80$ e (b) $w=0,90$.

Na Figura 4.16 ilustramos, de maneira particularizada, as duas fases distintas que o modelo pode apresentar para redes com $N=10000$ eleitores e $N_{c}=10$ candidatos. 


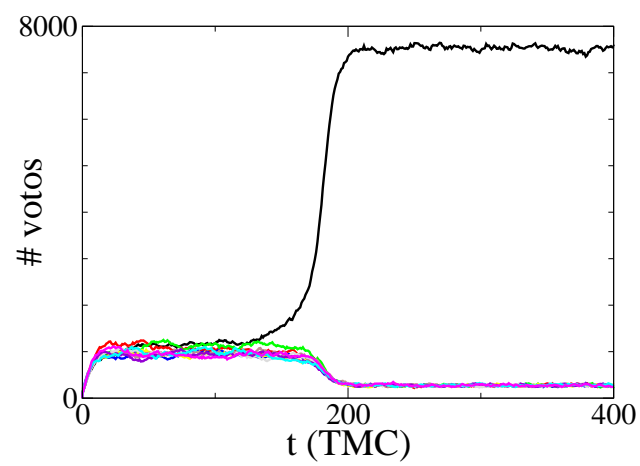

(a)

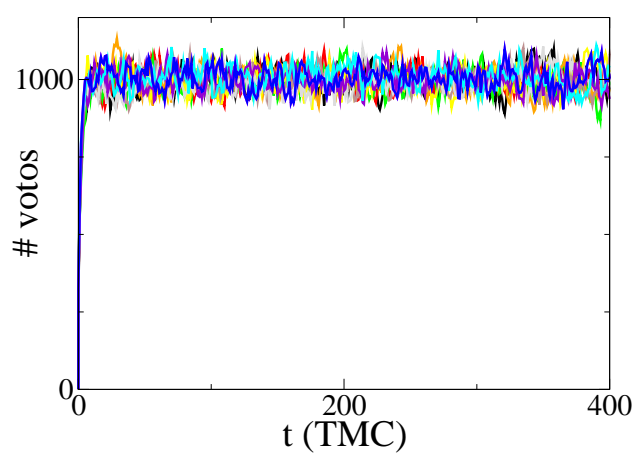

(b)

Figura 4.16: Evolução temporal da distribuição de votos para o Modelo Sznajd Complexo com Ruído numa Rede de Barabási-Albert com $N=10000$ eleitores e $N_{c}=10$ candidatos. (a) Fase ordenada $(w=0.1)$ e (b) Fase Desordenada $(w=0.9)$.

As séries temporais estão de acordo com os cálculos realizados no capítulo 3, indicando a existência de uma transição de fase em função da intensidade do ruído. Do ponto de vista quantitativo, apresentamos, na Figura 4.17, uma comparação entre a estimativa de campo médio e os resultados numéricos. Esta situação indica que o campo médio funciona 
como uma estimativa superior para os valores exatos da quantidade de votos que cada candidato pode assumir.

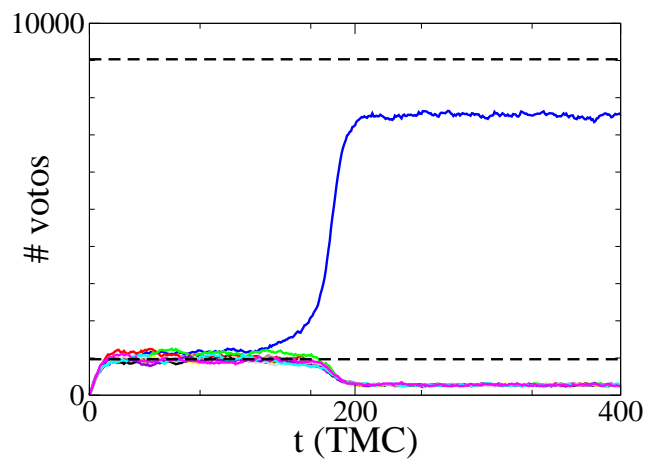

(a)

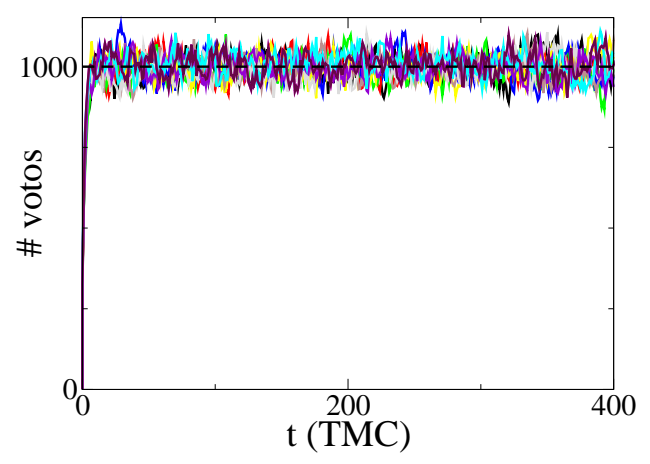

(b)

Figura 4.17: Evolução temporal da distribuição de votos para o Modelo Sznajd Complexo com Ruído numa Rede de Barabási-Albert com $N=10000$ eleitores e $N_{c}=10$ candidatos. As linhas tracejadas representam os valores de campo médio para o estado estacionário do modelo. (a) $w=0,10$ e (b) $w=0,90$.

Na Figura 4.18 destacamos o fato de que a aproximação tende a 
tornar-se cada vez melhor quando a intensidade do ruído tende a zero $\left(w \ll w_{t}\right)$. Para intensidades do ruído acima do ponto crítico, a estimativa de campo médio perde o caráter de estimativa superior e pode ser interpretada como a média temporal do número de votos por candidato. 


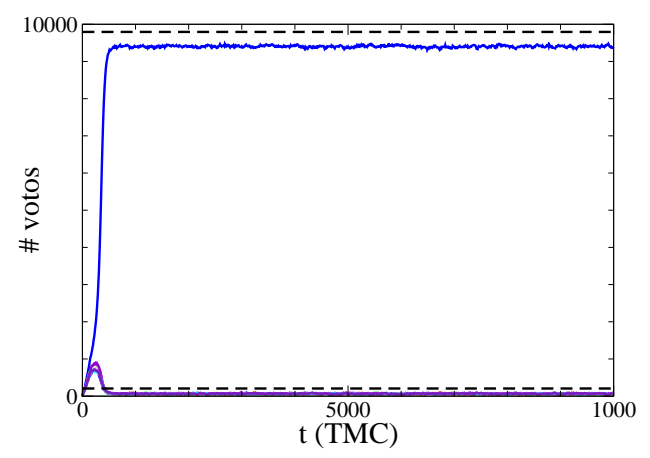

(a)

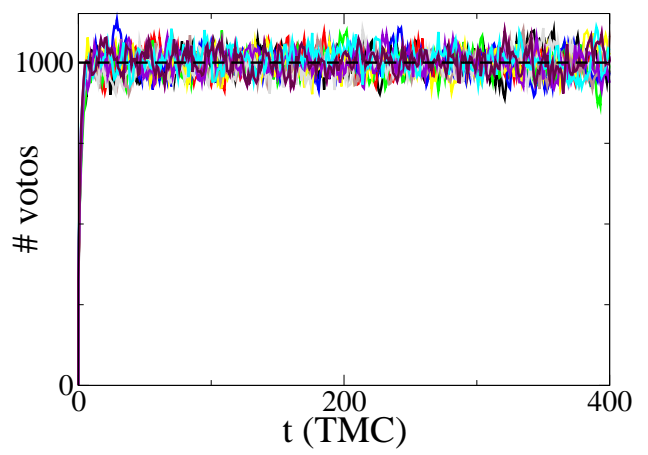

(b)

Figura 4.18: Evolução temporal da distribuição de votos para o Modelo Sznajd Complexo com Ruído numa Rede de Barabási-Albert com $N=10000$ eleitores e $N_{c}=10$ candidatos. As linhas tracejadas representam os valores de campo médio para o estado estacionário do modelo. (a) $w=0,025$ e (b) $w=0,975$.

A reinterpretação do campo médio para a fase ruidosa é consequência direta do empate técnico apresentado pelos candidatos que impõem, para a média temporal de votos por candidato, a condição $\langle n\rangle=\frac{N}{N_{c}}=1000$, com o número de votos de todos os candidatos 
flutuando em torno deste valor.

Perceba também que a aproximação de campo médio distingue o comportamento dos candidatos minoritários do comportamento do candidato vencedor, representado-os como uma média de seu comportamento coletivo, conforme as hipóteses assumidas em seu estabelecimento. Na próxima seção, formalizaremos este caráter da aproximação de campo médio quando interpretarmos físicamente as soluções instáveis apresentadas pelo diagrama de bifurcação numérico do modelo.

\subsubsection{Diagrama de Bifurcação e Transição de Fases na Rede de Barabási-Albert}

Para a construção do diagrama de bifurcação numérico do modelo é necessário tomarmos algumas precauções relacionadas ao tratamento dos candidatos minoritários. Na Figura 4.19, por exemplo, representamos o comportamento típico do candidato majoritário e da média dos demais candidatos no estado estacionário da dinâmica. Tomamos o valor médio dos candidatos minoritários para possibilitar uma comparação com os resultados de campo médio, como está indicado na Figura 4.20. 


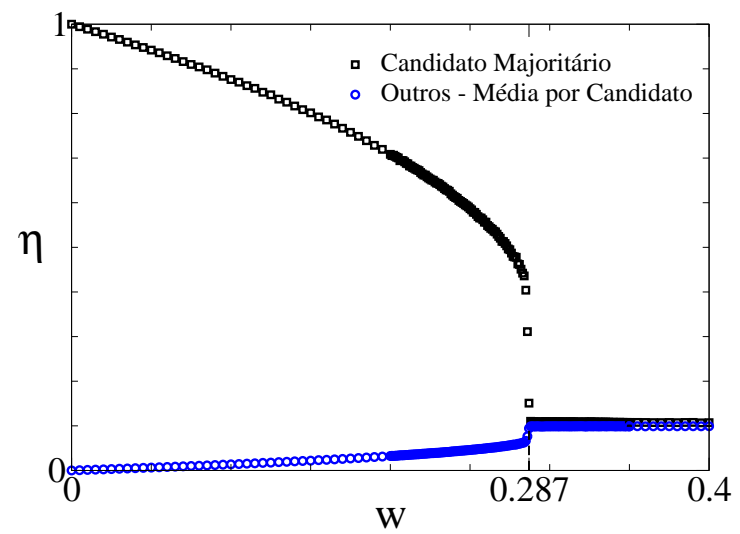

Figura 4.19: Diagrama de bifurcação numérico para o Modelo Sznajd Complexo com Ruído numa Rede de Barabási-Albert com $N=10000$ eleitores e $N_{c}=10$ candidatos.

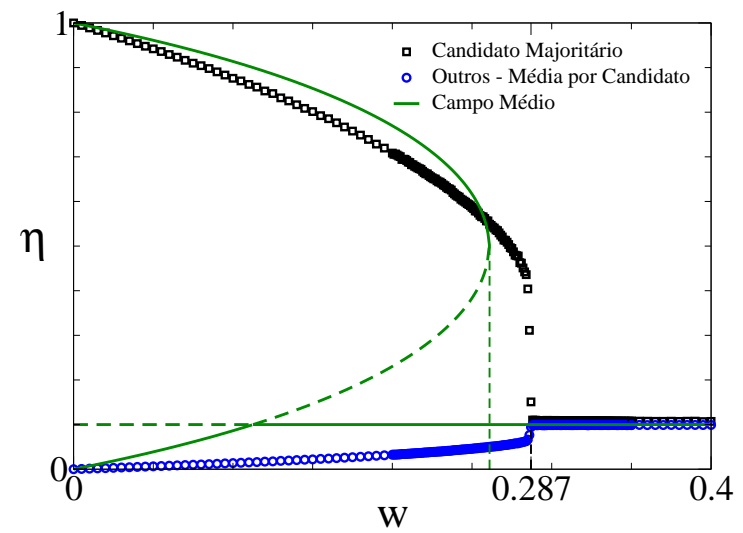

Figura 4.20: Comparação entre os diagramas de bifurcação numérico e analítico para o Modelo Sznajd Complexo com Ruído numa Rede de Barabási-Albert com $N=10000$ eleitores e $N_{c}=10$ candidatos. 
É importante destacar que os valores indicados no diagrama não satisfazem à condição de normalização, entretanto, os valores devem satisfazer $\eta^{\sigma_{v}}+\left(N_{c}-1\right) \eta^{\sigma^{\prime}} \approx 1$, onde $\eta^{\sigma^{\prime}}$ representa a média dos candidatos minoritários, ou seja, satisfazem, em média, a condição de normalização.

Para a construção do diagrama analítico utilizamos os parâmetros médios $\left\langle k_{v}\right\rangle$ e $\langle l\rangle$ da rede obtidos através de simulações, com um ensemble de 100 realizações da dinâmica. Sendo assim, apesar de utilizarmos a expressão algébrica oriunda da aproximação de campo médio, podemos nos referir a esta construção e suas consequências como semianalítica. Na Tabela 4.16 apresentamos os resultados numéricos para os parâmetros médios de uma rede BA estabelecidos por esta dissertação (Araújo e Prado - 2011) e os comparamos com resultados publicados na dissertação de Vannucchi (Vannucchi e Prado - 2006)[6].

\begin{tabular}{|c|c|c|c|c|}
\hline Rede BA & $\langle k\rangle$ & $\left\langle k_{v}\right\rangle$ & $\langle l\rangle$ & $\left\langle k^{\prime}\right\rangle$ \\
\hline Araújo & $10,0 \pm 0,1$ & $30,0 \pm 1,0$ & $0,108 \pm 0,001$ & $256,3 \pm 8,5$ \\
\hline Vannucchi & 10 & 25,3 & - & - \\
\hline
\end{tabular}

Tabela 4.16: Resultados numéricos para os parâmetros médios da rede de Barabási-Albert. Os valores médios foram estabelecidos a partir de um ensemble com 100 realizações numéricas de redes com $N=10000$ vértices e conectividade mínima $m=5$.

Como esperado, o valor do fator de acoplamento é muito menor do que a unidade $(\langle l\rangle \ll 1)$ e está associado ao fato das transições de segunda espécie serem pouco frequentes. Além disso, $\langle k\rangle=2 m$, $\left\langle k_{v}\right\rangle>\langle k\rangle$ e $\left\langle k^{\prime}\right\rangle \gg\langle k\rangle$ refletem a propriedade de conexão preferencial das redes de Barabási-Albert.

Destacamos ainda que a curva semi-analítica está sempre acima da curva numérica, corroborando a expectativa de que os resultados de 
campo médio funcionam com uma estimativa superior dos resultados exatos. A comparação entre as curvas referentes aos candidatos minoritários torna esta expectativa bastante nítida.

Outra possibilidade muito interessante pode ser explorada ao considerarmos o candidato majoritário juntamente com o total de votos dos candidatos minoritários, de acordo com as figuras 4.21 e 4.22.

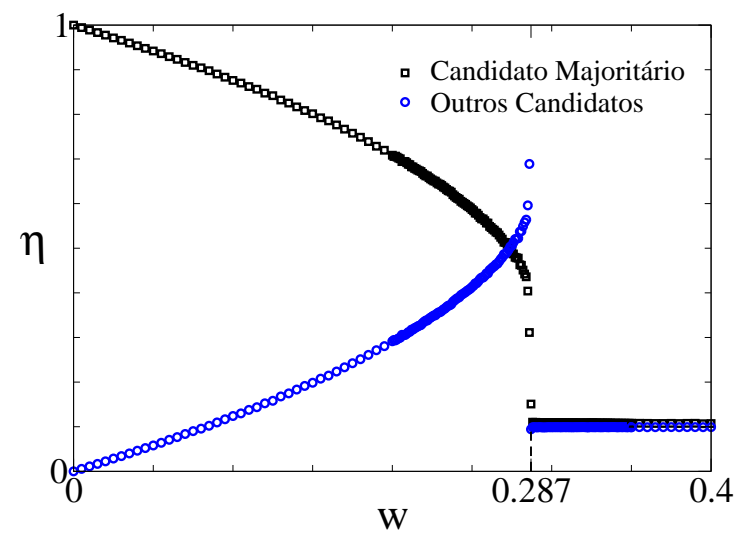

Figura 4.21: Diagrama de bifurcação para o Modelo Sznajd Complexo numa Rede de Barabási-Albert com $N=10000$ eleitores e $N_{c}=10$ candidatos. Para a construção do diagrama consideramos o candidato majoritário e a soma dos votos dos demais candidatos. 


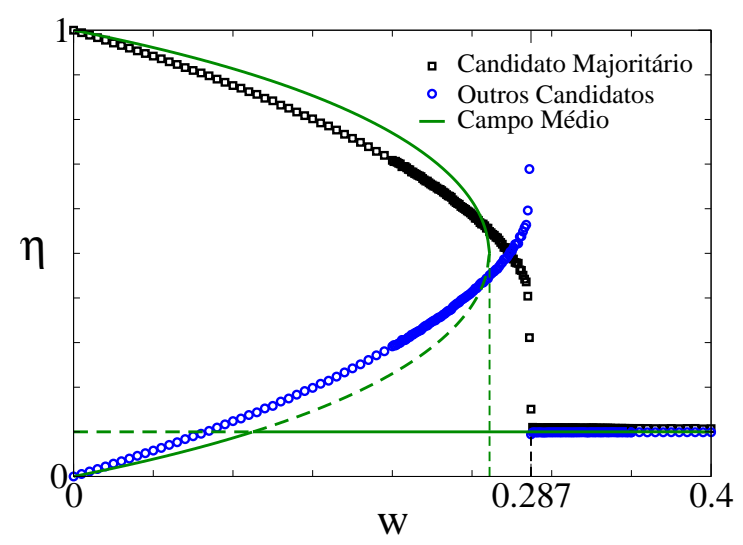

Figura 4.22: Comparação entre os diagramas de birfucação numérico e analítico para o Modelo Sznajd Complexo numa Rede de Barabási-Albert com $N=10000$ eleitores e $N_{c}=10$ candidatos. Para a construção dos diagramas consideramos o candidato majoritário e a soma dos votos dos demais candidatos.

O ponto de intersecção entre as curvas numéricas indica que é possível ao sistema um cenário onde o candidato vitorioso não detém a maioria absoluta dos votos, diferentemente da aproximação de campo médio, onde o candidato vitorioso sempre vence com mais da metade das intenções de votos $\left(\eta_{C M}^{\sigma_{v}} \geq \frac{1}{2}\right)$.

A intensidade do ruído associada à intersecção entre as curvas é igual a $w^{\prime}=0,275 \pm 0,001$. Além disso, $w_{t}^{(C M)}<w^{\prime}<w_{t}$, indicando que o ponto crítico obtido via campo médio fornece uma estimativa inferior ao valor exato.

De fato, os resultados que obtemos para o ponto crítico foram $w_{t}^{(C M)}=0,261 \pm 0,002$ e $w_{t}=0,287 \pm 0,001$, apresentando aceitável concordância entre si, em contraste com os resultados de Vannucchi que foram $w_{t}^{(C M)}=0,089 \pm 0,001$ e $w_{t}=0,313 \pm 0,002$. É nesse 
sentido que afirmamos ter resolvido as discrepâncias encontradas na abordagem de Vannucchi, com uma melhoria significativa da estimativa analítica do ponto crítico. A seguir, na Tabela 4.17, estabelecemos uma comparação entre os resultados para o ponto crítico obtidos nesta dissertação, os resultados de Vannucchi e os resultados de campo médio para as demais redes estudadas.

\begin{tabular}{|c|c|c|}
\hline Rede & - & $w_{t}$ \\
\hline \multirow{2}{*}{$\begin{array}{c}\text { Rede BA } \\
\text { Araújo e Prado, 2011 } \\
\text { Rede BA } \\
\text { Vannucchi e Prado, 2006 }\end{array}$} & Numérico & $0,287 \pm 0,001$ \\
\cline { 2 - 3 } & Analítico & $0,261 \pm 0,002$ \\
\hline Grafo & Analítico & $0,089 \pm 0,001$ \\
\hline Completo & Numérico & - \\
\cline { 2 - 3 } Cadeia de & Analítico & 0,357 \\
\hline Eleitores & Numérico & - \\
\cline { 2 - 3 } Rede & Analítico & 0,294 \\
\hline Quadrada & Numérico & - \\
\cline { 2 - 3 } Rede & Analítico & 0,327 \\
\hline Cúbica & Numérico & - \\
\cline { 2 - 3 } Redes Regulares & Analítico & 0,337 \\
\hline \multirow{2}{*}{\begin{tabular}{c} 
Altas Dimensionalidades \\
\cline { 2 - 3 }
\end{tabular}} & Numérico & - \\
\cline { 2 - 3 } & Analítico & 0,357 \\
\hline
\end{tabular}

Tabela 4.17: Intensidades críticas de ruído para as diferentes redes estudadas. Para o estabelecimento dos resultados na rede BA nos utilizamos de um ensemble com 100 realizações de redes com $N=10000$ vértices e $N_{c}=10$ candidatos.

Para finalizarmos, apresentaremos o parâmetro de ordem do modelo na rede de Barabási-Albert através das figuras 4.23 e 4.24, destacando a transição de fase sob o ponto de vista numérico. Desta forma, confirmamos a expectativa quanto ao sistema exibir uma transição de fases 
descontínua gerada pelos cálculos de campo médio do capítulo anterior.

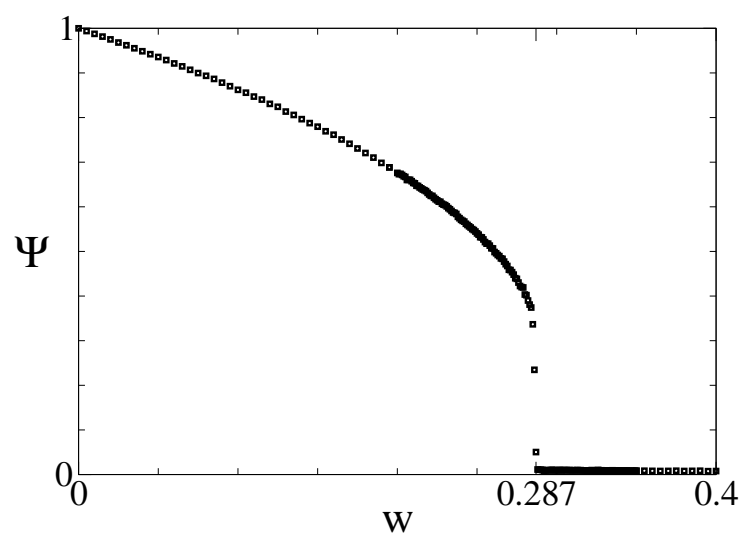

Figura 4.23: Parâmetro de ordem numérico em função do parâmetro de controle para o Modelo Sznajd Complexo com Ruído numa Rede de BarabásiAlbert com $N=10000$ eleitores e $N_{c}=10$ candidatos. O modelo apresenta uma transição de fases de primeira ordem entre as fases de consenso e a fase ruidosa com ponto crítico igual a $w_{t}=0,287 \pm 0,001$ 


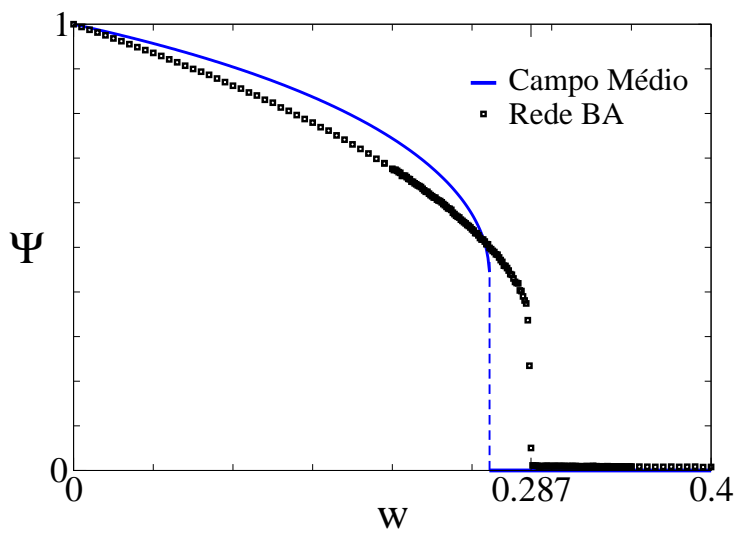

Figura 4.24: Comparação entre o parâmetro de ordem numérico e o parâmetro de ordem analítico em função do parâmetro de controle para o Modelo Sznajd Complexo com Ruído numa Rede de Barabási-Albert com $N=10000$ eleitores e $N_{c}=10$ candidatos. 


\section{Capítulo 5}

\section{Conclusões e Perspectivas}

Neste trabalho, estudamos o Modelo Sznajd Complexo numa abordagem predominantemente analítica, diferentemente das abordagens geralmente publicadas, pretendendo assim fornecer uma contribuição original ao problema. Apresentamos uma equação mestra que descreve a evolução de opiniões do modelo e determinamos seus estados estacionários através de uma aproximação de campo médio para diferentes redes, com especial ênfase para a rede de Barabási-Albert.

As discrepâncias encontradas entre o resultado de campo médio e o resultado de simulação numérica de Vannucchi e Prado foram resolvidas com a inclusão de novos termos na equação mestra. Esses termos, que haviam sido desprezados antes por serem supostamente muito raros, têm importância significativa e devem ser considerados.

Nosso estudo da versão sem ruído do Modelo Sznajd Complexo não apresentou grandes surpresas, sempre evoluindo para um estado estacionário de consenso entre os eleitores, da mesma forma que o Modelo Sznajd original. Simulações numéricas na rede de Barabási-Albert com $N=10000$ eleitores e $N_{c}=10$ candidatos apotam para o estado de consenso, tipicamente, a partir de 30 tempos de Monte Carlo. Na versão do modelo com ruído, que corresponde a aplicação de um campo 
externo, investigamos o efeito de campanhas publicitárias no processo eleitoral. Nestas circunstâncias, o campo introduz flutuações na opinião do eleitorado que destroem o estado de consenso, determinando um comportamento mais rico ao modelo.

A aproximação de campo médio aplicada a este caso leva a estados estacionários que correspondem ou a vitória isolada de algum candidato (um único candidato detêm a grande maioria dos votos) ou ao empate técnico entre todos os candidatos (os candidatos apresentam, em média, a mesma quantidade de votos). É razoável que para campos fracos, a situação de vitória isolada prevaleça, enquanto que, para campos muito fortes, as flutuações sejam tão intensas que façam prevalecer a situação de empate técnico. Sendo assim, dependendo da intensidade do campo externo aplicado, o sistema pode sofrer uma transição de fase, e passamos a interpretar os estados estacionários de vitória isolada e empate técnico como as fases ordenada e desordenada, respectivamente, que caracterizam o sistema.

Os cálculos fornecem um diagrama de bifurcação para o estado estacionário da dinâmica que permite o estabelecimento formal da transição de fases, e, o fato deste apresentar uma descontinuidade sugere que a transição de fases seja de primeira ordem. Uma grande quantidade de simulações para redes de Barabási-Albert corroboram com o caráter descontínuo da transição. É importante ressaltar que a descontinuidade do diagrama depende do número de candidatos, o que faz surgir a possibilidade de uma transição de segunda ordem para o caso particular de dois candidatos $\left(N_{c}=2\right)$.

O diagrama de birfucação motivou a definição do parâmetro de ordem como a diferença entre os patamares das fases ordenada e desordenada, a menos de um constante multiplicativa conveniente posta a fim de normalizar o parâmetro. Desta forma, definimos o parâmetro de 
ordem do modelo como sendo $\psi=\left(\frac{N_{c}}{N_{c}-1}\right)\left(\eta_{+}^{\sigma}-\eta_{0}^{\sigma}\right)$ e a intensidade crítica do campo externo é identificada como sendo $w_{t}$ (valor crítico do parâmetro de controle). A reprodução numérica do parâmetro de ordem apresentou boa concordância quando confrontada com os resultados analíticos, sendo que a intensidade crítica do campo externo encontrada em simulações realizadas numa rede de Barabási-Albert foi $w_{t}=0,287 \pm 0,001$, enquanto que o resultado encontrado numa aproximação de campo médio foi $w_{t}=0,261 \pm 0,002$.

Apesar da ênfase atribuída às redes de Barabási-Albert, muitos resultados para um grafo completo e para redes regulares com diferentes dimensionalidades foram estabelecidos. Estas redes funcionaram como um "toy model", de maneira a ilustrar o cálculo dos parâmetros médios da rede e dos parâmetros críticos, tornando-se um importante referencial no estabelecimento do demais resultados. Além disso, verificamos que um grafo completo no limite termodinâmico e uma rede regular no limite de altas dimensionalidades descrevem igualmente o comportamento do modelo por tratarem de casos em que as correlações espaciais são descartadas de forma semelhante.

Portanto, a abordagem de campo médio, apesar de muito simples, mostra-se bastante eficiente na descrição de grande parte do comportamento dinâmico do modelo, de maneira que o objetivo de caracterizá-lo analticamente foi alcançado com êxito.

Entretanto, devemos reconhecer que ainda há muito a ser feito, com muitos desdobramentos da teoria a serem melhor explorados. Assim, temos muitas perspectivas de continuidade para este trabalho, dentre elas, desenvolver mais resultados numéricos em outros tipos de rede, pois representariam uma complementação muito importante dos resultados obtidos no capítulo 3 e de investigar a sensibilidade dos parâmetros críticos em relação a variações nos parâmetros médios da 
rede.

Além disso, pretendemos estimar analiticamente os parâmetros médios da rede de Barabási-Albert, enriquecendo os resultados do capítulo 4, e determinando seus parâmetros críticos por um método exclusivamente analítico. A conjectura a respeito das propriedades do modelo em redes regulares no limite de altas dimensionalidades deve ser revista, formalizando os cálculos analíticos que permitam efetivamente sua prova.

Finalmente, muitos outros temas como, por exemplo, a identificação do candidato vencedor e a descrição mais precisa de eleições majoritárias devem ser investigados. 


\section{Apêndice A}

\section{Construindo a Rede de Barabási-Albert}

Neste apêndice iremos detalhar vários pedaços do algoritmo empregado por nós para a construção da rede de Barabási-Albert.

Processo de Construção da Rede:

1. Inicialmente, satisfazendo a regra de conectividade mínima $m$ entre os sítios, tomamos $m+1$ sítios onde todos se conectam entre si.

2. Para a adição de um novo sítio à rede, sorteamos uma aresta de forma equiprovável, e, em seguida, sorteamos um dos sítios desta aresta, satisfazendo a regra de conexão preferencial. Repetimos esta etapa um número suficiente de vezes até satisfazer o critério de conectividade mínima novamente.

3. Procedemos assim, sucessivamente, até que a rede apresente $\mathrm{N}$ sítios.

Características do Ponteiro de Representação de Arestas ** M: 
1. Número de colunas: 2 - associado aos pares ordenados que correspondem às arestas da rede.

2. Número de linhas: $a(m, N)=m(m+1) / 2+m[N-(m+1)]$

O trecho do código fonte responsável pela construção da rede de Barabási-Albert é apresentado nas figuras A.1 e A.2, e um exemplo desta representação, como resultado de uma saída padrão do programa é apresentado na Figura A.3.

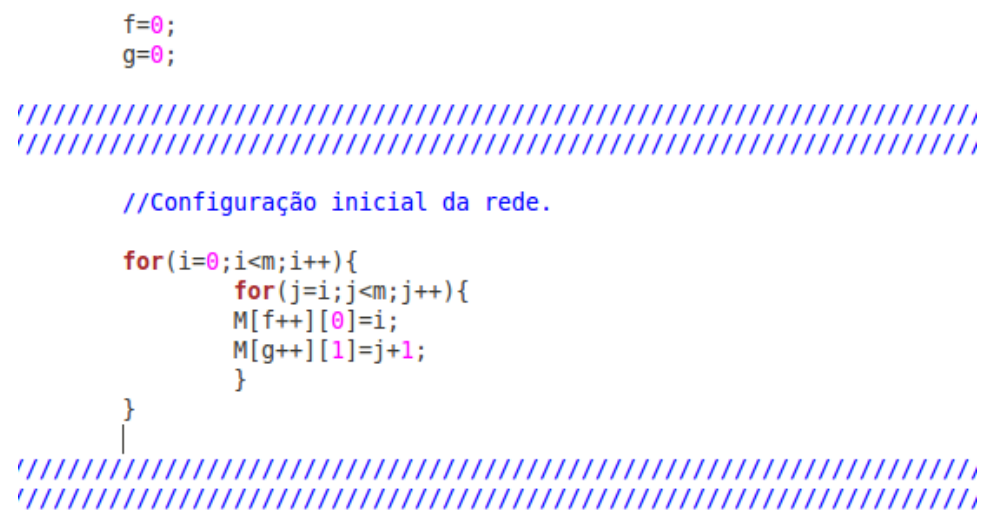

Figura A.1: Parte do código fonte responsável pela construção da configuração inicial da rede de Barabási-Albert. 


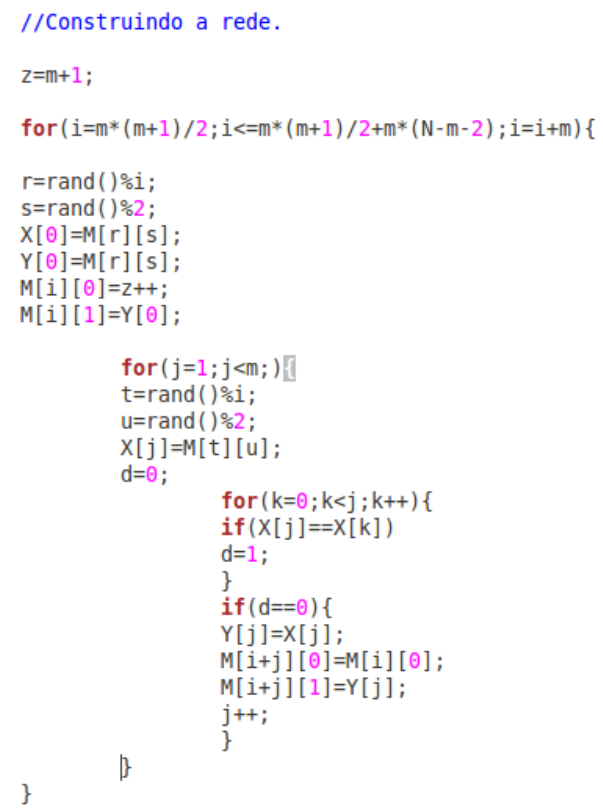

Figura A.2: Parte do código fonte responsável pela continuidade na construção da rede de Barabási-Albert. 


$\begin{array}{cll}1 & \theta & 1 \\ 2 & \theta & 2 \\ 3 & 1 & 2 \\ 4 & & \\ 5 & 3 & 0 \\ 63 & 1 \\ 7 & 4 & 0 \\ 84 & 1 \\ 95 & 2 \\ 105 & 1 \\ 11 & 6 & 4 \\ 12 & 6 & 5 \\ 137 & 6 \\ 147 & 0 \\ 15 & 8 & 3 \\ 16 & 8 & 2 \\ 179 & 2 \\ 18 & 9 & 8 \\ 19 & 10 & 6 \\ 20 & 10 & 1\end{array}$

Figura A.3: Saída padrão do ponteiro ${ }^{* *}$ M ilustrando uma rede de BarabásiAlbert $N=11$ vértices e conectividade mínima $m=2$. A linha 4 destaca a configuração inicial do restante da rede. 


\section{Apêndice B}

\section{Ponteiro de Opiniões}

Exemplo típico para a saída do algoritmo responsável pela construção do Ponteiro de Opiniões *Op.

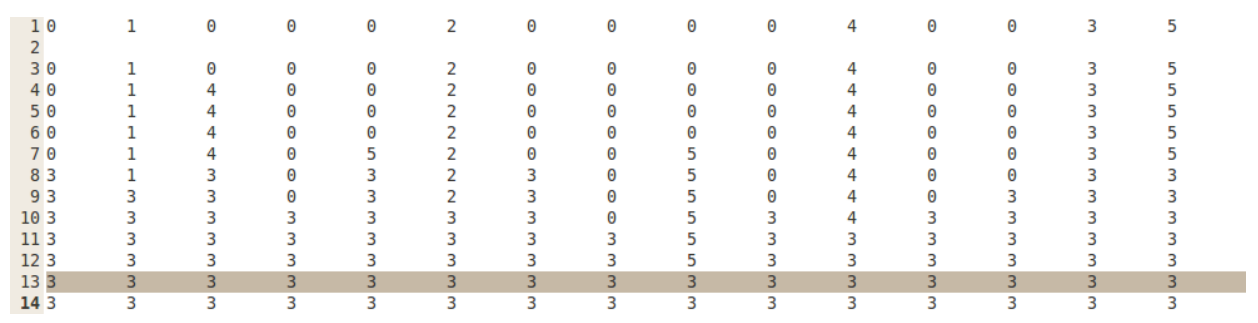

Figura B.1: Saída padrão para o ponteiro de opiniões de uma rede de Barabási-Albert com $N=15$ e $N_{c}=5$. A linha 1 representa a configuração da rede correspondente ao processo de semeadura e a linha hachurada indica que a dinâmica atingiu o estado estacionário de consenso com todos os eleitores escolhendo o candidato 3. Cada linha está associada a um instante medido em Tempo de Monte Carlo. 


\section{Apêndice C}

\section{Representação de Vizinhos}

Processo de Construção da Representação de Vizinhos:

1. Escolhida uma aresta, por exemplo $(0,1)$, acrescentamos uma unidade ao número de vizinhos de cada um de seus sítios mediante um ponteiro ${ }^{*} \mathrm{Q}$ que controla o grau dos sítios. Em seguida, colocamos um na lista de vizinhos do outro determinando o ponteiro $* * \mathrm{~A}$.

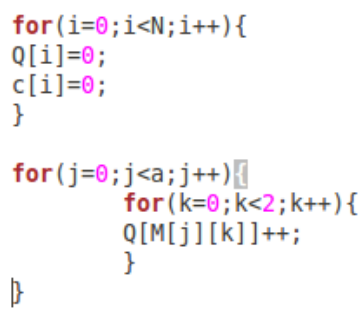

Figura C.1: Trecho do código fonte que contabiliza o grau de cada vértice. Q[i] é o grau do vértice i. 


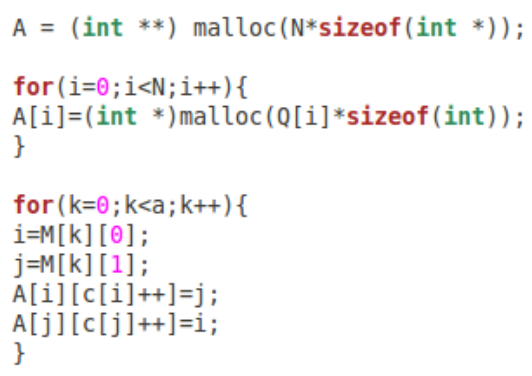

Figura C.2: Trecho do código fonte responsável pela construção da Representação de Vizinhos.

$\begin{array}{cll}1 & 0 & 5 \\ 2 & 1 & 6 \\ 3 & 2 & 5 \\ 4 & 3 & 3 \\ 5 & 4 & 3 \\ 6 & 5 & 3 \\ 7 & 6 & 4 \\ 8 & 7 & 2 \\ 9 & 8 & 3 \\ 10 & 9 & 2 \\ 11 & 10 & 2\end{array}$

Figura C.3: Saída padrão do ponteiro ${ }^{*} \mathrm{Q}$ para a rede de Barabási-Albert com $m=2$ e $N=11$ apresentada anteriormente na Figura A.3. O elemento da primeira coluna de cada linha representa um dado sítio da rede e o elemento da segunda coluna o seu respectivo grau, por exemplo, o sítio 0 tem grau $Q[0]=5$. 


$\begin{array}{rrrrrrr}1 & 1 & 2 & 3 & 4 & 7 & \\ 20 & 2 & 3 & 4 & 5 & 10 \\ 30 & 1 & 5 & 8 & 9 & \\ 40 & 1 & 8 & & & \\ 50 & 1 & 6 & & & \\ 62 & 1 & 6 & & & \\ 7 & 4 & 5 & 7 & 10 & & \\ 86 & 0 & & & & \\ 93 & 2 & 9 & & & \\ 10 & 2 & 8 & & & & \\ 11 & 6 & 1 & & & & \end{array}$

Figura C.4: Saída padrão do ponteiro **A para a rede de Barabási-Albert com $m=2$ e $N=11$ apresentada anteriormente na Figura A.3. 


\section{Apêndice D}

\section{Representação de Número de Votos}

Apresentamos a seguir, uma saída padrão do ponteiro *Nvotos, exemplificando a Representação de Número de Votos.

\begin{tabular}{|c|c|c|c|c|c|c|}
\hline 10 & 10 & 1 & 1 & 1 & 1 & 1 \\
\hline 2 & & & & & & \\
\hline 31 & 10 & 1 & 1 & 1 & 1 & 1 \\
\hline 42 & 9 & 1 & 1 & 1 & 2 & 1 \\
\hline 53 & 9 & 1 & 1 & 1 & 2 & 1 \\
\hline 64 & 9 & 1 & 1 & 1 & 2 & 1 \\
\hline 75 & 7 & 1 & 1 & 1 & 2 & 3 \\
\hline 86 & 5 & 1 & 1 & 6 & 1 & 1 \\
\hline 97 & 4 & $\theta$ & 1 & 8 & 1 & 1 \\
\hline 108 & 1 & $\theta$ & $\theta$ & 12 & 1 & 1 \\
\hline 119 & $\theta$ & $\theta$ & $\theta$ & 14 & $\theta$ & 1 \\
\hline 1210 & 0 & $\theta$ & $\theta$ & 14 & $\theta$ & 1 \\
\hline 1311 & $\theta$ & $\theta$ & $\theta$ & 15 & 0 & $\theta$ \\
\hline 1412 & $\theta$ & $\theta$ & $\theta$ & 15 & $\theta$ & $\theta$ \\
\hline
\end{tabular}

Figura D.1: Saída padrão do ponteiro *Nvotos para uma rede de BarabásiAlbert com $N=15$ e $N_{c}=5$. A coluna 1 indica o instante particular da dinâmica, a coluna 2 indica o número de indecisos e as demais colunas representam cada candidato em ordem de enumeração. 


\section{Apêndice E}

\section{Acompanhando a Dinâmica do Modelo Sznajd Complexo}

Neste apêndice, vamos considerar por simplicidade, uma rede de Barabási-Albert com $N=5$ eleitores e $N_{c}=2$ candidatos numa dinâmica sem ruído e utilizaremos a Representação de Opiniões por se tratar de uma representação bastante apropriada para acompanharmos a dinâmica passo-a-passo, apesar do inconveniente de não podermos visualizar as interações entre os sítios. Convém informar ainda que alteramos o código fonte do programa para que indicasse explicitamente o sítio escolhido, o vizinho sorteado e o que se ocorreu após a interação. 


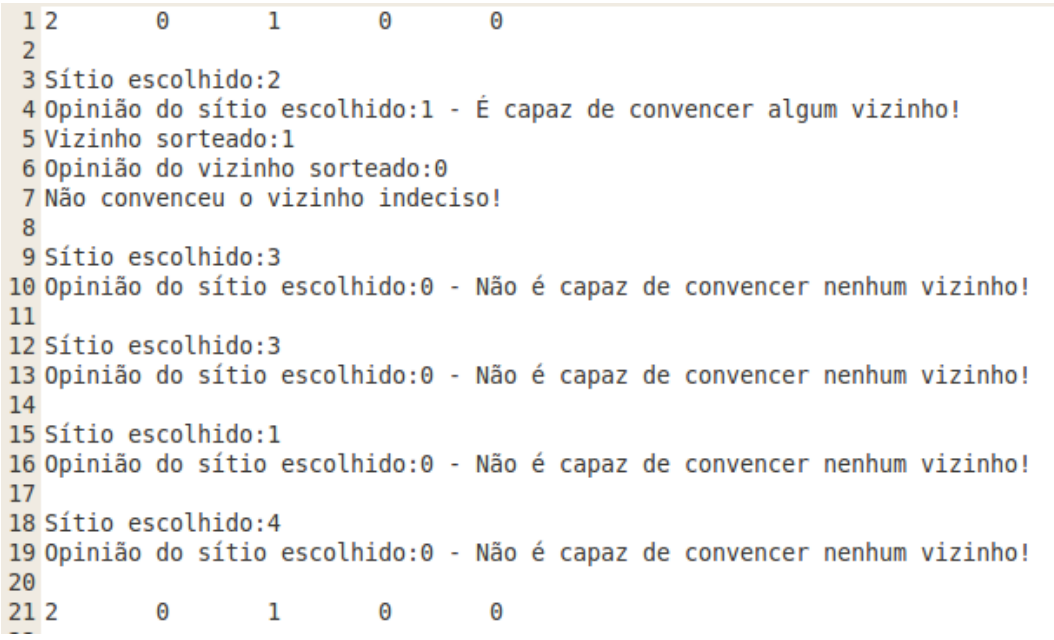

Figura E.1: Saída padrão referente ao ponteiro * Op ilustrando a dinâmica do Modelo Sznajd em seu primeiro Tempo de Monte Carlo. O fato da rede possuir $N=5$ eleitores determina as 5 interações entre os sítios correspondentes a cada Passo de Monte Carlo.

Nesta primeira ilustração (Figura E.1), representamos o primeiro Tempo de Monte Carlo da dinâmica. A primeira linha representa o instante 0 TMC referente ao processo de semeadura, com os sítios 0 e 2 "semeados". Mais uma vez, perceba que ficamos impossibilitados de visualizar explicitamente a vizinhança de cada sítio.

É natural que, no início, a configuração não se altere ou sofra alterações a taxas muito lentas em virtude da grande quantidade de sítios indecisos que predominam sobre a rede (no caso em questão, a linha 21 evidencia que a configuração do sistema permaneceu inalterada). Ressaltamos ainda que, no primeiro Passo de Monte Carlo, apesar do sítio escolhido ser capaz de convencer algum vizinho, ele não consegue efetivar o convencimento pois o vizinho assediado pode rejeitar o confronto direto e permanecer indeciso com probabilidade complementar ao inverso da conectividade do sítio escolhido. 
No instante seguinte, $t=2$ TMC, o sistema sofre sua primeira modificação no terceiro Passo de Monte Carlo com o sítio 2 convencendo o sítio 1 num confronto direto como indicado na Figura E.2. No primeiro passo, a configuração não sofreu alterações por representar o confronto direto entre dois vizinhos discordantes.

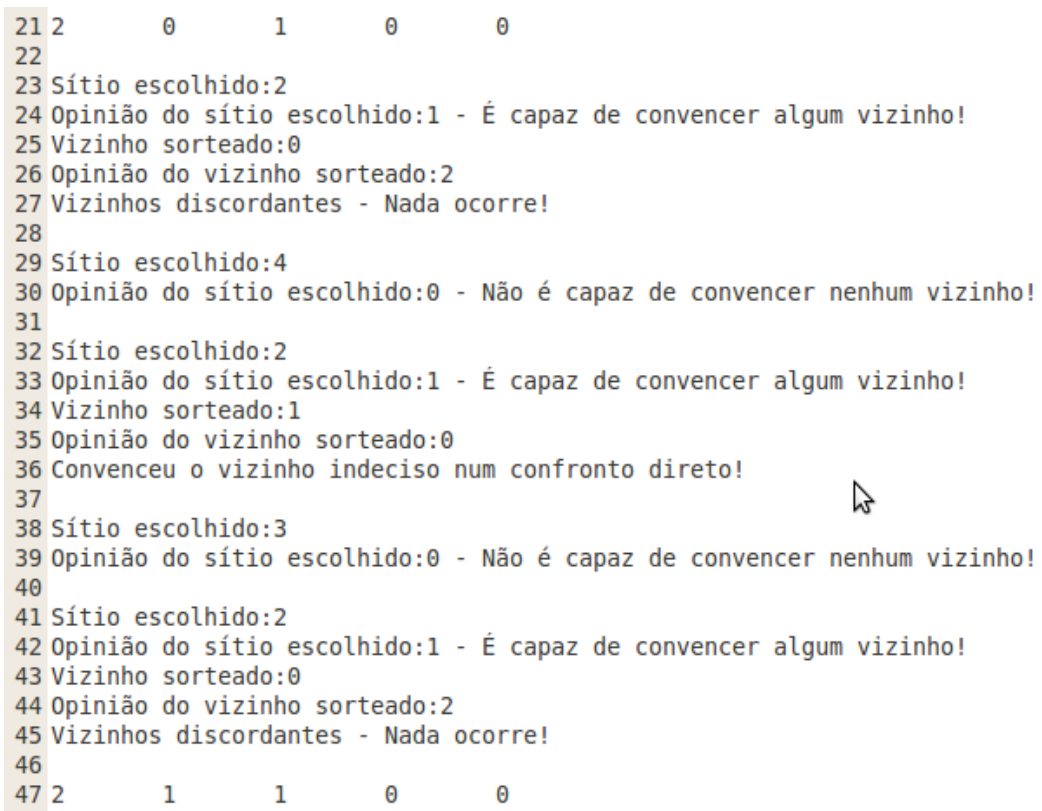

Figura E.2: Saída padrão referente ao ponteiro ${ }^{*}$ Op ilustrando o segundo instante da dinâmica iniciada na Figura E.1.

O instante $t=3$ TMC é marcado por uma nova modificação da configuração em que o sítio 1 convence o sítio 4, num confronto direto, a votar no candidato 1 . Neste instante, o candidato 1 passa a predominar sobre a rede e começa a definir sua vitória. Acompanhe através da próxima figura (Figura E.3). 


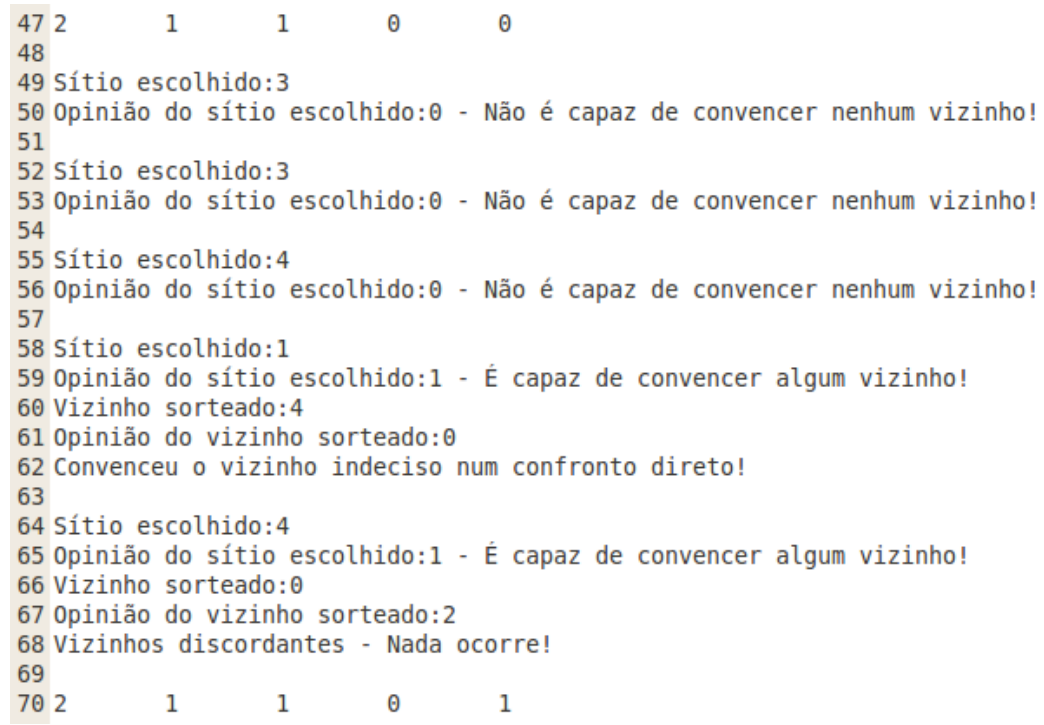

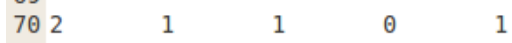

Figura E.3: Saída padrão referente ao ponteiro *Op ilustrando o terceiro instante da dinâmica iniciada na Figura E.1.

A predominância de eleitores partidários à opinião 1 é manisfestada com a ocorrência mais frequente de interações entre vizinhos concordantes a partir do instante $t=4$ TMC, de acordo com a Figura E.4. 


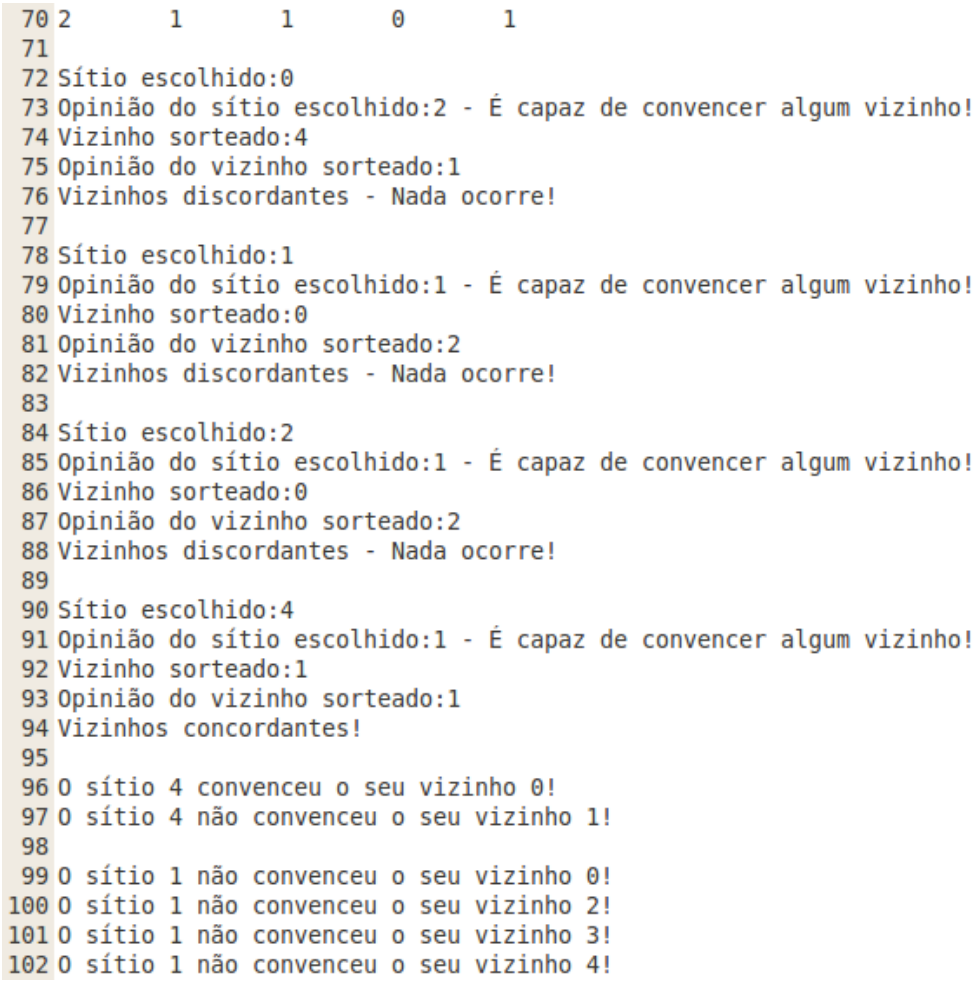

Figura E.4: Saída padrão referente ao ponteiro *Op ilustrando o quarto instante da dinâmica iniciada na Figura E.1.

No último Passo de Monte Carlo de $t=4$ TMC, o sítio 4 conseguiu convencer o sítio 0 a votar no candidato 1 ao assediar todos os sítios de sua vizinhança, a saber, os sítios 0 e $1\left(\Gamma_{4}=\{0,1\}\right)$, por outro lado, o sítio 1 não conseguiu convencer nenhum de seus vizinhos em sua investida $\left(\Gamma_{1}=\{0,2,3,4\}\right)$. Este resultado era esperado pois, como podemos perceber, a probabilidade do sítio 4 convencer algum de seus vizinhos é igual a $\frac{1}{2}$, enquanto que a probabilidade do sítio 1 convencer seus vizinhos é bem menor e vale $\frac{1}{4}$.

Desta forma, o candidato 1 consolida sua vitória ao eliminar a última intenção de voto ao candidato 2 , impedindo qualquer reação 
por parte deste candidato.

Em $t=5 \mathrm{TMC}$, as ocorrências de interações entre vizinhos concordantes passam a ser muito mais frequentes do que no instante $t=3$ TMC. Em nosso exemplo, a configuração permaneceu inalterada neste instante da dinâmica.

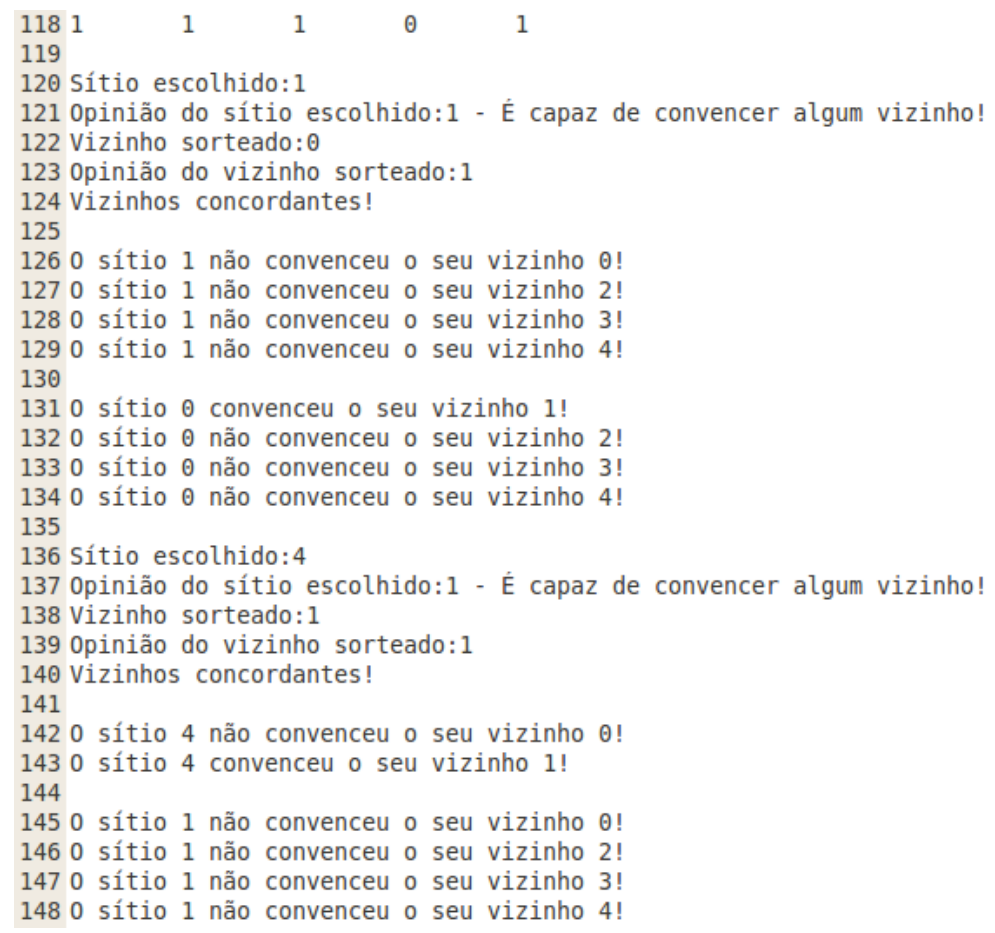

Figura E.5: Saída padrão referente ao ponteiro *Op ilustrando o quinto instante da dinâmica iniciada na Figura E.1. É notável o aumento na frequência de interações entre vizinhos concordantes.

Finalmente, a partir do instante $t=6 \mathrm{TMC}$, os eleitores indecisos são extintos e a dinâmica atinge seu estado estacionário de consenso com todos os eleitores votando no candidato 1. Ver Figura E.6. 


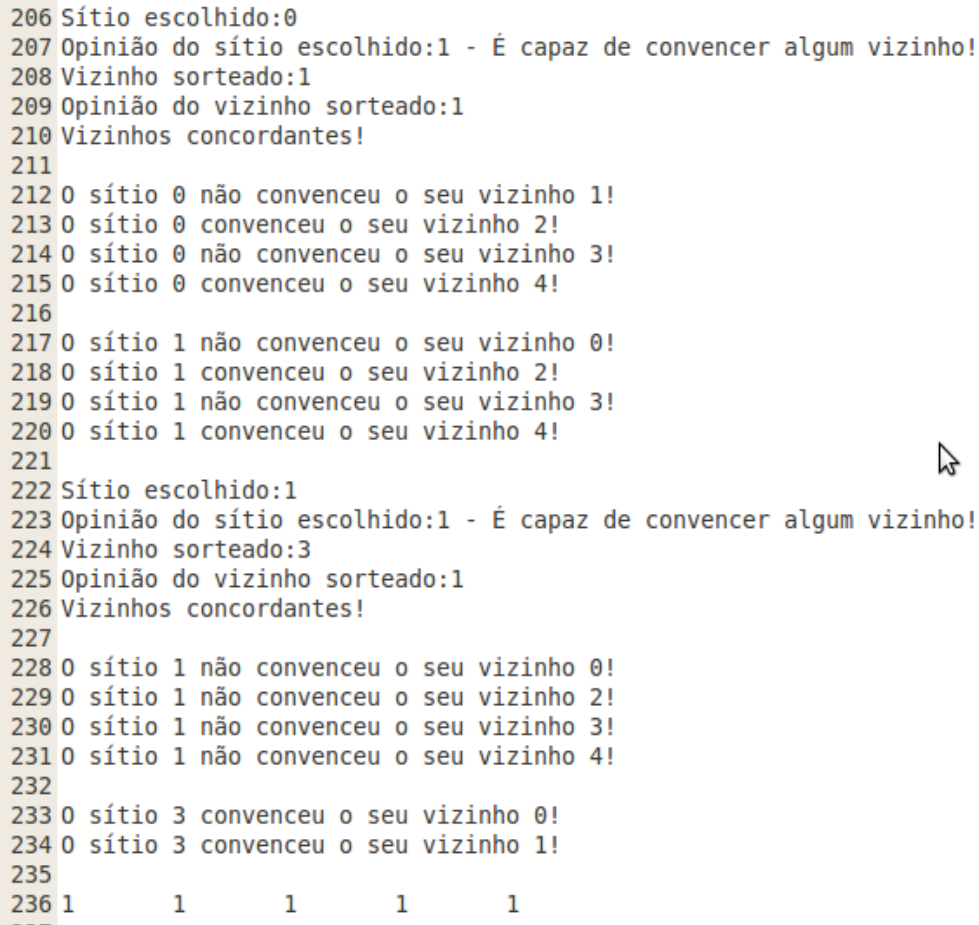

Figura E.6: Saída padrão referente ao ponteiro ${ }^{*}$ Op ilustrando o sexto instante da dinâmica iniciada na Figura E.1. A partir deste instante, a dinâmica atinge seu estado estacionário de consenso com todos os eleitores votando no candidato 1 .

Nas próximas ilustrações, pretendemos explicitar a interferência do ruído à dinâmica do modelo. Por coerência, vamos considerar as mesmas condições utilizadas no caso anterior, com a mesma rede e o mesmo número de candidatos, sendo a inclusão de um ruído com intensidade $w=0.1$ o único diferencial.

A Figura E.7 destaca a atuação do ruído logo nos primeiros instantes da dinâmica. No terceiro Passo de Monte Carlo do instante $t=1 \mathrm{TMC}$, o sítio 3, até então indeciso, deixa de obedecer a dinâmica convencional e escolhe votar no candidato 1 ao ser influenciado pela propaganda. 


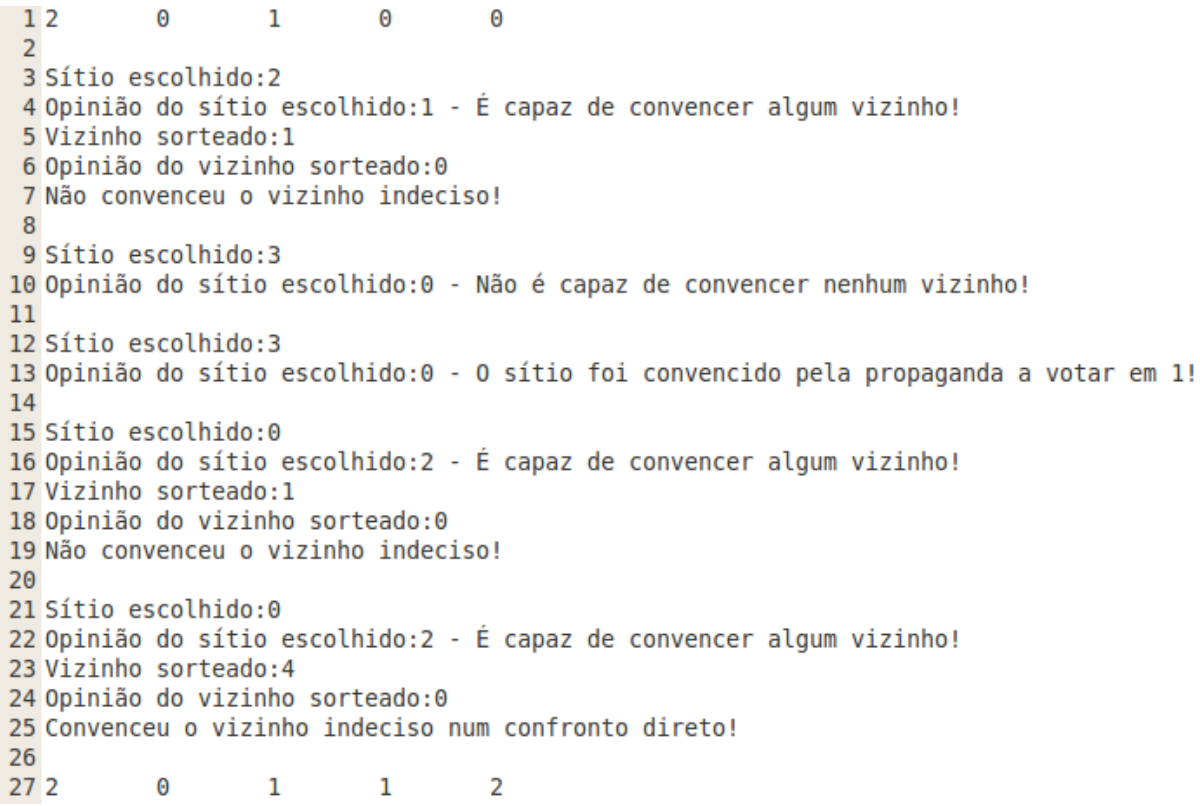

Figura E.7: Representação de Opiniões do instante $t=1$ TMC para 0 Modelo Sznajd Complexo com Ruído numa rede de Barabási-Albert com $N=5$ eleitores, $N_{c}=2$ candidatos e $w=0,1$.

Em seguida, a Figura E.8 mostra que, no instante $t=6$ TMC, o sistema assume um estado de consenso com todos os eleitores votando no candidato 2. Entretanto, diferentemente do caso anterior, esta configuração não é estacionária, pois, é possível a cada eleitor, com probabilidade w, mudar espontaneamente de opinião e o número de votos de um candidato flutua com o tempo. A figura E.9 mostra, no instante $t=8$ TMC, a destruição deste estado de consenso com a retomada do candidato 1 pelo eleitor 3 . 


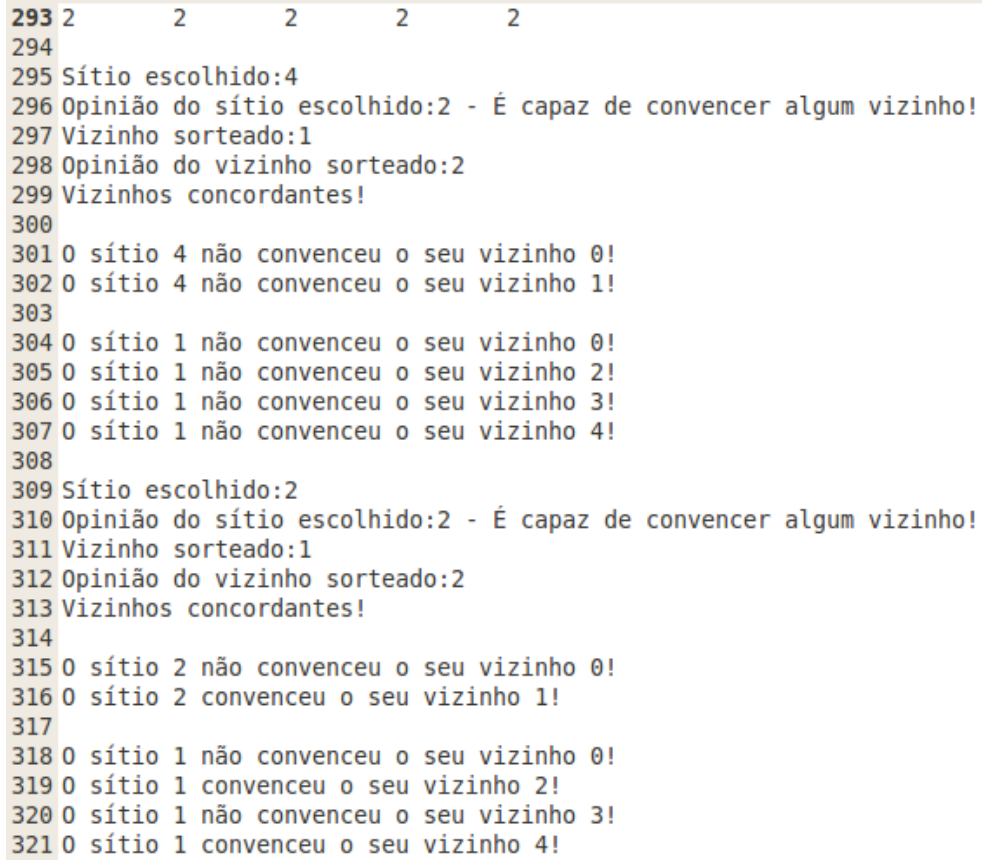

Figura E.8: Representação de Opiniões do instante $t=6$ TMC para o Modelo Sznajd Complexo com Ruído numa rede de Barabási-Albert com $N=5$ eleitores, $N_{c}=2$ candidatos e $w=0,1$. Neste instante, o sistema assume um temporário estado de consenso. 


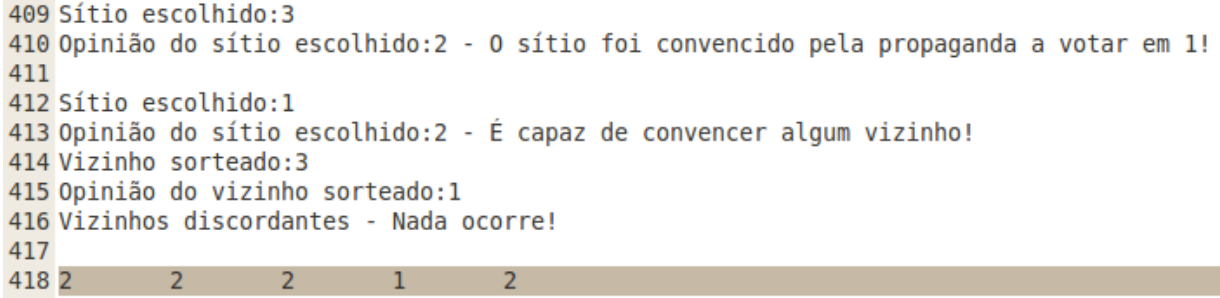

Figura E.9: Representação de Opiniões do instante $t=8$ TMC para o Modelo Sznajd Complexo com Ruído numa rede de Barabási-Albert com $N=5$ eleitores, $N_{c}=2$ candidatos e $w=0,1$. A linha hachurada destaca a destruição do estado de consenso assumido pelo sistema no instante $t=6$ TMC.

Para finalizar, analisemos o caso extremo em que a intensidade do ruído é máxima (noise=1) numa rede de Barabási-Albert nas mesmas condições dos exemplos anteriores. Como esperado, em todos os Passos de Monte Carlo de um instante qualquer, a dinâmica sofre a interferência do ruído e a dinâmica convencional é completamente abandonada (ver Figura E.10). Evidentemente, no estado estacionário, os candidatos apresentam, em média, a mesma quantidade de votos como mostra a Figura E.11. 


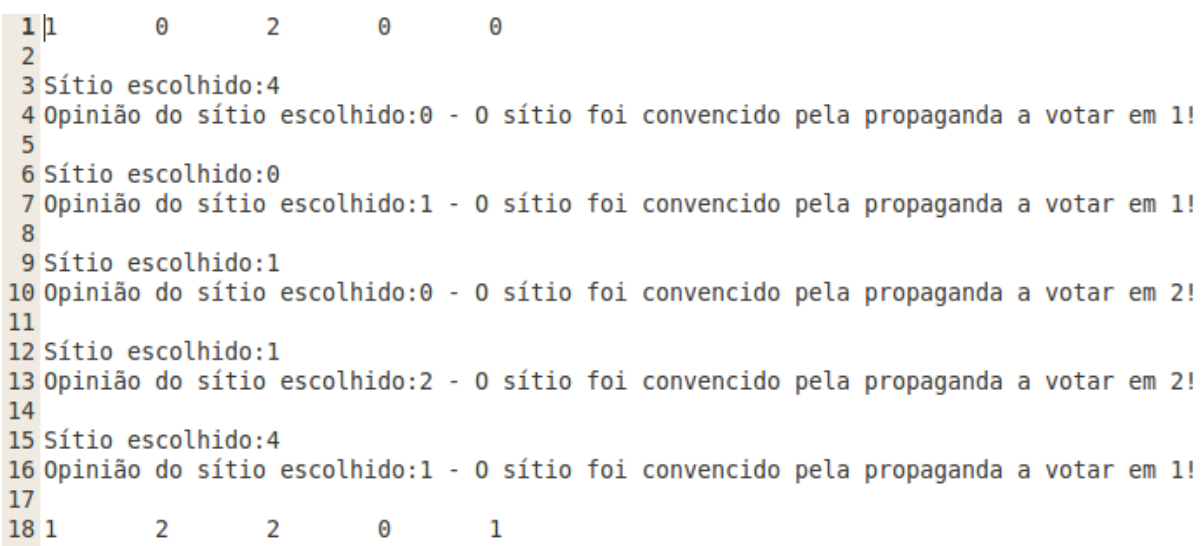

Figura E.10: Representação de Opiniões para o Modelo Sznajd Complexo com Ruído Máximo em seus instantes iniciais. Perceba que a dinâmica convencional é completamente abandonada. 


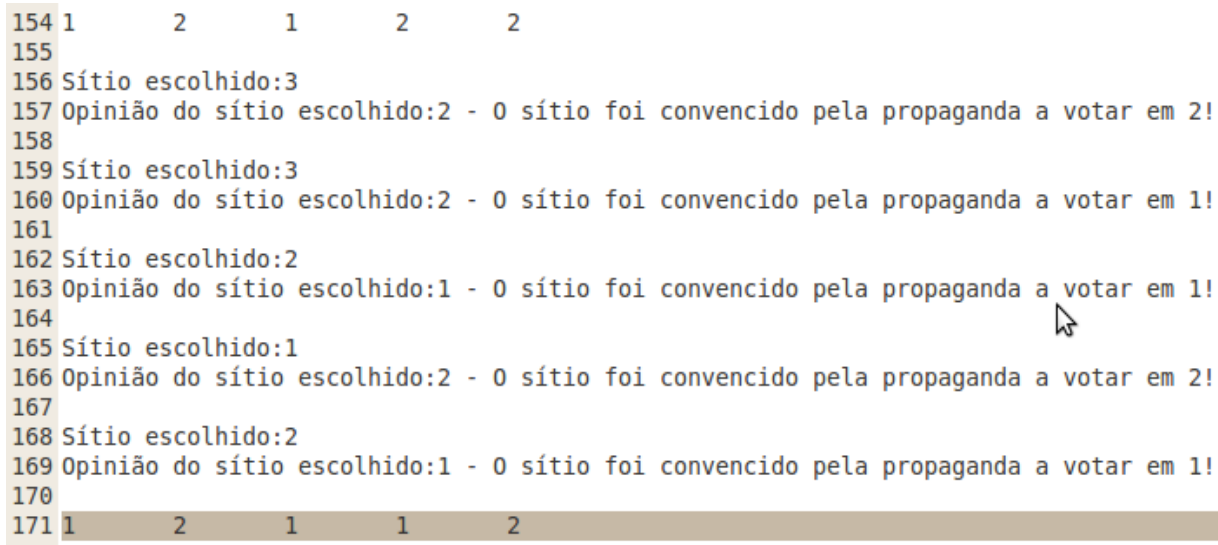

Figura E.11: A linha hachurada destaca a Representação de Opiniões para o Modelo de Sznajd Complexo numa rede de Barabási-Albert com $N=5$ eleitores, $N_{c}=2$ candidatos e $w=1$ no instante $t=10$ TMC. O intervalo de tempo decorrido desde o instante inicial até o instante considerado é longo o suficientemente para considerarmos o sistema em regime estacionário. Perceba que, aproximadamente, os dois candidatos apresentam a mesma quantidade de votos.

As fases de vitória isolada (fase ordenada) e empate técnico (fase desordenada) que o sistema pode assumir são identificadas, respectivamente, nas figuras E.9 e E.11. 


\section{Apêndice F}

\section{Algoritmo Utilizado no Cálculo dos Parâmetros Médios da Rede}

Algoritmo de Monte Carlo utilizado para o cálculo dos parâmetros médios de uma rede de Barabási-Albert com $N=10000$ vértices e conectividade mínima $m=5$ arestas por vértice em Linguagem C.

\#include $\langle$ stdio.h $\rangle$

\#include math.h〉

\#include $\langle$ stdlib.h $\rangle$

\#define Nc 10

\#define N 10000

\#define $\mathrm{m} 5$

\#define a $(\mathrm{m}+1) * \mathrm{~m} / 2+(\mathrm{N}-(\mathrm{m}+1)) * \mathrm{~m}$

//Modelo de Sznajd em uma rede BA com N vértices e conectividade mínima m. 


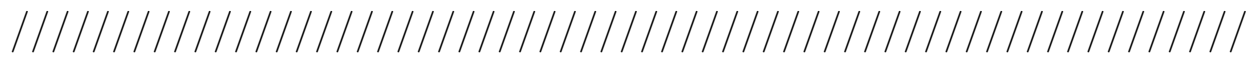

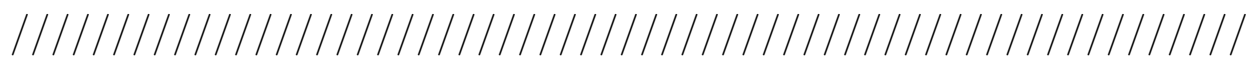

//Declaração de variáveis.

int main(int argc, char $* \operatorname{argv}[])\{$

int ${ }^{*} * \mathrm{M},{ }^{*} \mathrm{Q},{ }^{*} \mathrm{q},{ }^{*} \mathrm{Q} 2,{ }^{*} \mathrm{c},{ }^{*} \mathrm{P},{ }^{*} \mathrm{X},{ }^{*} \mathrm{Y},{ }^{*} \mathrm{E},{ }^{*} \mathrm{~F},{ }^{*} \mathrm{H},{ }^{*} \mathrm{~S},{ }^{*} \mathrm{w},{ }^{*} \mathrm{Op}$, *sem;

int b, g, G, d, f, i, r, s, t, u, j, k, l, p, z, D, Z, h, n, v, maior, x, s3, r0, r1, dsem;

int sv, sort0, sort1, sort2, sort3;

float W1, wt, t1, sv2, sv3, wsort, km, tm, xx, Nf;

float ${ }^{*} \mathrm{w} 1,{ }^{*} \mathrm{H} 2$;

int ${ }^{* *} \mathrm{~A},{ }^{* *} \mathrm{~B},{ }^{* *} \mathrm{C}$;

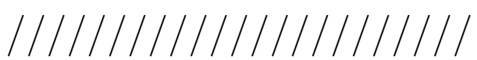

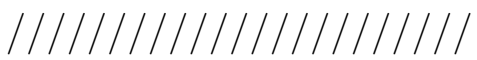

$$
\begin{aligned}
& \mathrm{M}=\left(\text { int }^{* *}\right) \operatorname{malloc}\left(\mathrm{a}^{*} \operatorname{sizeof}\left(\text { int }^{*}\right)\right) \text {; } \\
& \text { for }(i=0 ; i<a ; i++)\{ \\
& \mathrm{M}[\mathrm{i}]=(\text { int } *) \operatorname{malloc}\left(2^{*} \operatorname{sizeof}(\text { int })\right) \text {; } \\
& \} \\
& \mathrm{Q}=(\text { int } *) \operatorname{malloc}\left(\mathrm{N}^{*} \operatorname{sizeof}(\text { int })\right) \text {; } \\
& \mathrm{q}=(\text { int } *) \operatorname{malloc}\left(\mathrm{N}^{*} \operatorname{sizeof}(\text { int })\right) \text {; } \\
& \mathrm{Q} 2=(\text { int } *) \operatorname{malloc}\left(\mathrm{N}^{*} \operatorname{sizeof}(\mathrm{int})\right) \text {; } \\
& \mathrm{c}=(\text { int } *) \operatorname{malloc}\left(\mathrm{N}^{*} \operatorname{sizeof}(\text { int })\right) \text {; } \\
& \mathrm{P}=\left(\text { int }{ }^{*}\right) \operatorname{malloc}\left(\mathrm{N}^{*} \operatorname{sizeof}(\text { int })\right) \text {; } \\
& \mathrm{X}=\left(\text { int }^{*}\right) \operatorname{malloc}\left(\mathrm{m}^{*} \text { sizeof }(\text { int })\right) \text {; } \\
& \mathrm{Y}=(\text { int } *) \operatorname{malloc}\left(\mathrm{m}^{*} \operatorname{sizeof}(\text { int })\right) \text {; }
\end{aligned}
$$


$\mathrm{E}=\left(\right.$ int $\left.^{*}\right) \operatorname{malloc}\left(\mathrm{N}^{*}\right.$ sizeof $($ int $\left.)\right)$;

$\mathrm{F}=\left(\right.$ int $\left.^{*}\right) \operatorname{malloc}\left(\mathrm{N}^{*} \operatorname{sizeof}(\right.$ int $\left.)\right)$;

$\mathrm{H}=\left(\right.$ int $\left.^{*}\right) \operatorname{malloc}\left(\mathrm{N}^{*} \operatorname{sizeof}(\right.$ int $\left.)\right)$;

$\mathrm{S}=($ int $*) \operatorname{malloc}\left(\mathrm{N}^{*} \operatorname{sizeof}(\right.$ int $\left.)\right)$;

$\mathrm{w}=($ int $*) \operatorname{malloc}\left(\mathrm{N}^{*} \operatorname{sizeof}(\right.$ int $\left.)\right)$;

$\mathrm{Op}=($ int $*) \operatorname{malloc}\left(\mathrm{N}^{*}\right.$ sizeof $($ int $\left.)\right)$;

$\operatorname{sem}=\left(\right.$ int $\left.^{*}\right) \operatorname{malloc}\left(\mathrm{Nc}^{*} \operatorname{sizeof}(\right.$ int $\left.)\right)$;

Nvotos $=\left(\right.$ int $\left.^{*}\right) \operatorname{malloc}((\mathrm{Nc}+1) * \operatorname{sizeof}($ int $))$;

$\mathrm{w} 1=($ float $*) \operatorname{malloc}\left(\mathrm{N}^{*}\right.$ sizeof $($ float $\left.)\right)$;

$\mathrm{H} 2=($ float $*) \operatorname{malloc}\left(\mathrm{N}^{*}\right.$ sizeof $($ float $\left.)\right)$;

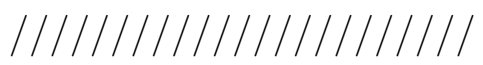

$1 / / / / / / / / / / / / / / / / / / 1$

FILE * resultados;

resultados = fopen("parametros_rede_ba.dat","w");

$\mathrm{f}=0$;

$\mathrm{g}=0$;

$\operatorname{srand}(\operatorname{atoi}(\operatorname{argv}[1]))$;

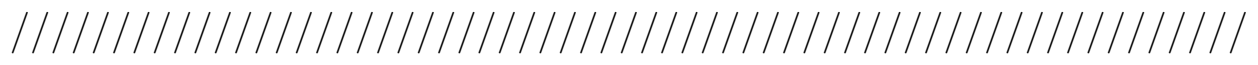

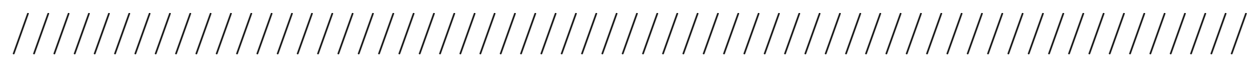

//Configuração inicial da rede.

$$
\begin{aligned}
& \text { for }(i=0 ; i<m ; i++)\{ \\
& \quad \operatorname{for}(j=i ; j<m ; j++)\{ \\
& \quad \mathrm{M}[\mathrm{g}++][0]=\mathrm{i}
\end{aligned}
$$




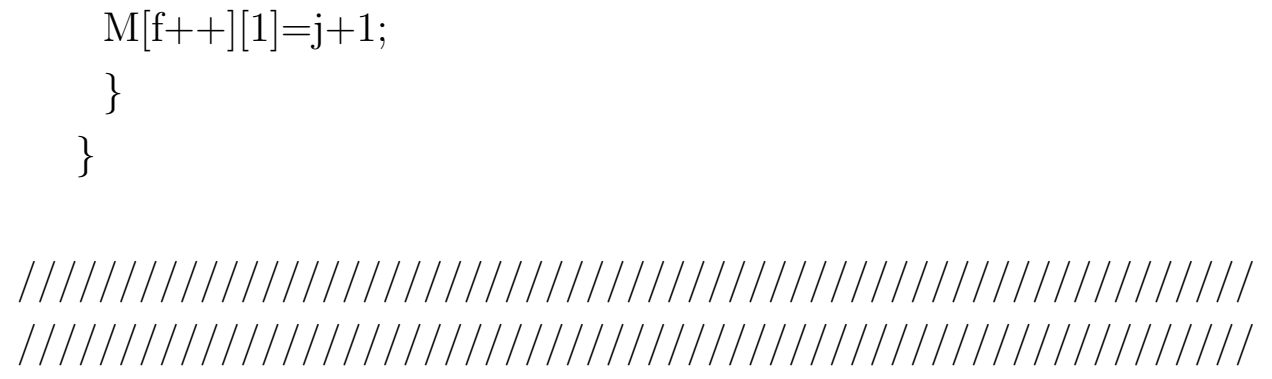

//Construindo a rede.

$\mathrm{z}=\mathrm{m}+1$

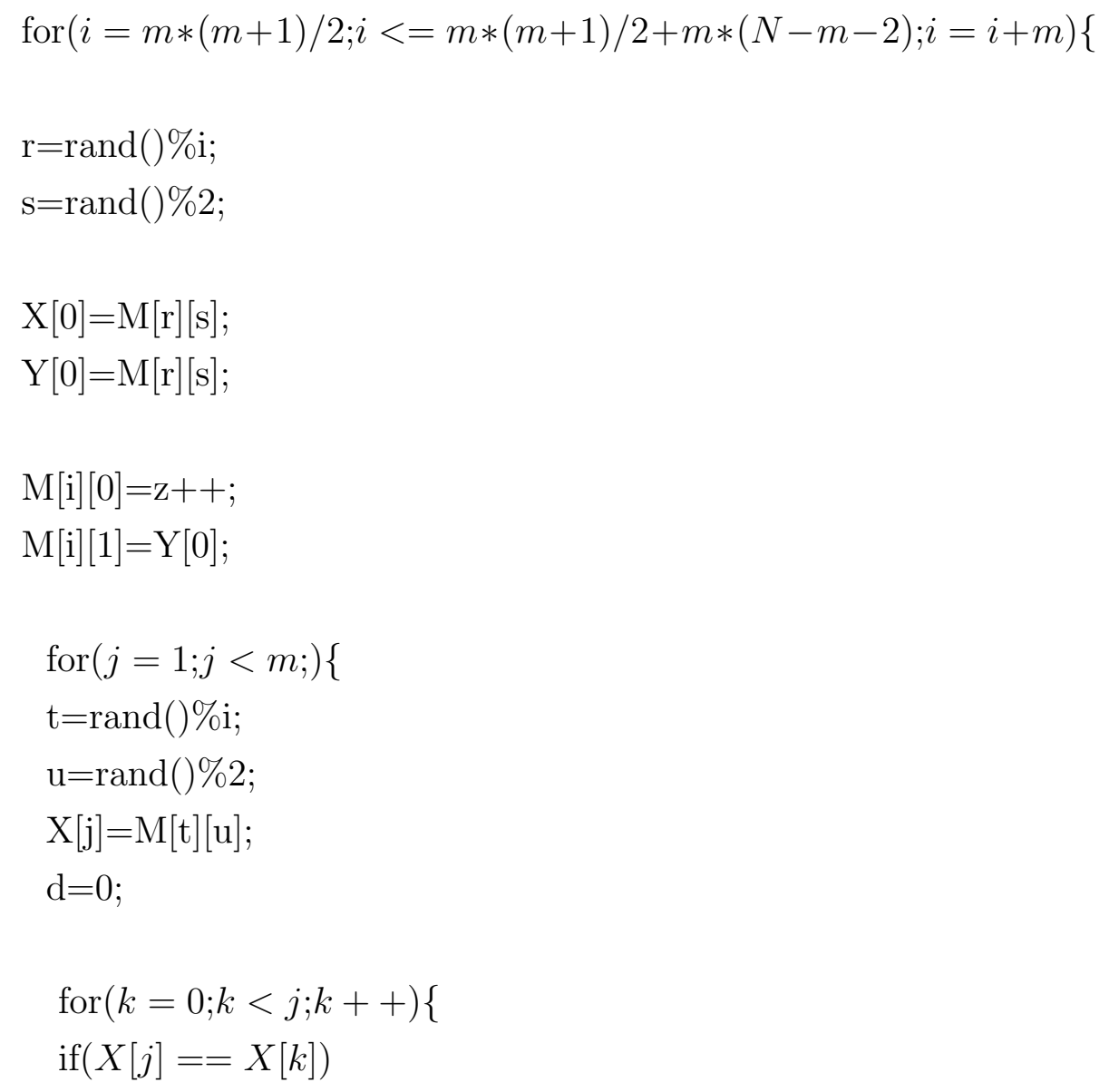




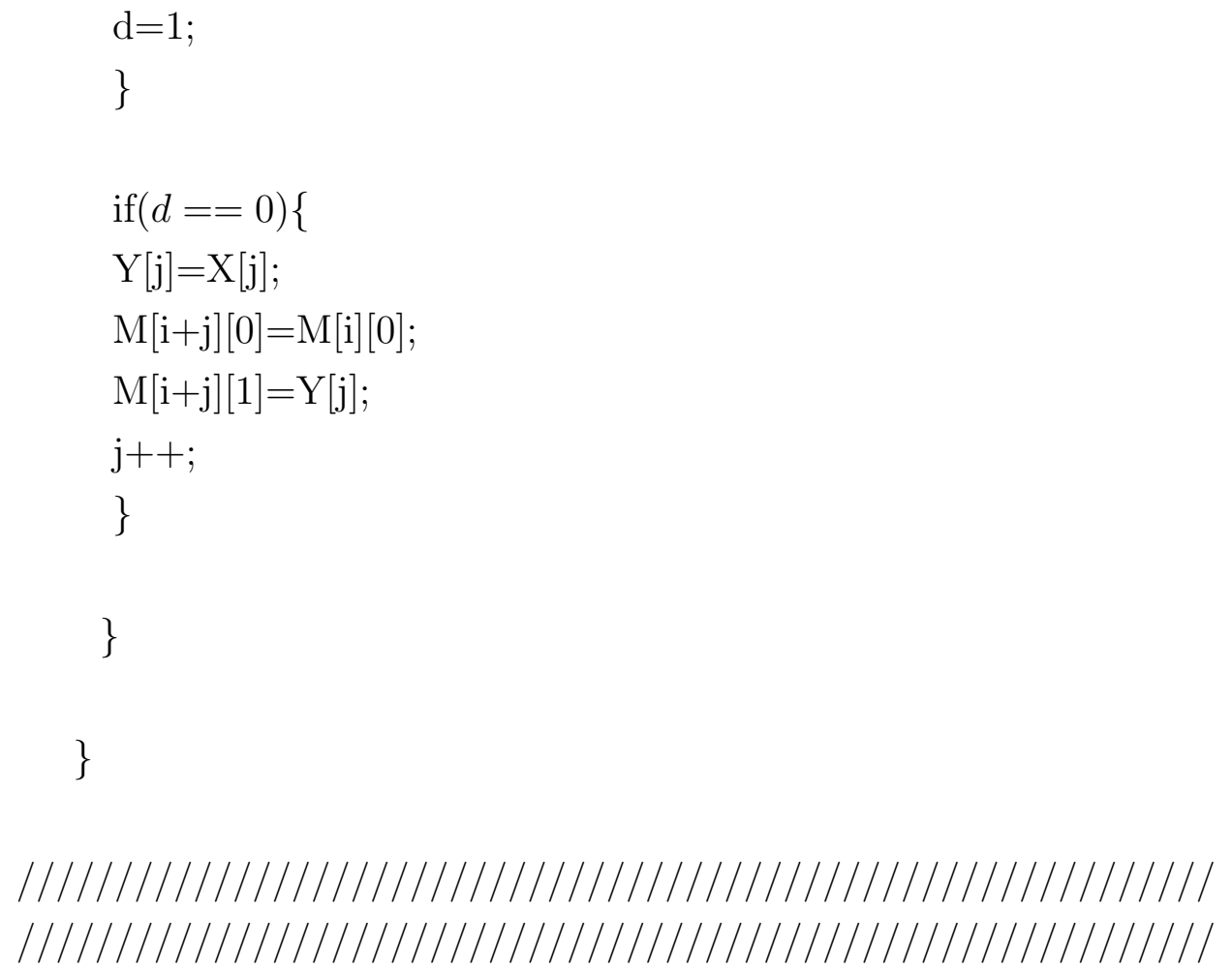

//Contabilizando o grau dos vértices.

$/ / \mathrm{Q}[\mathrm{i}]$ é o grau do vértice i.

//Cálculo de $\langle k\rangle$.

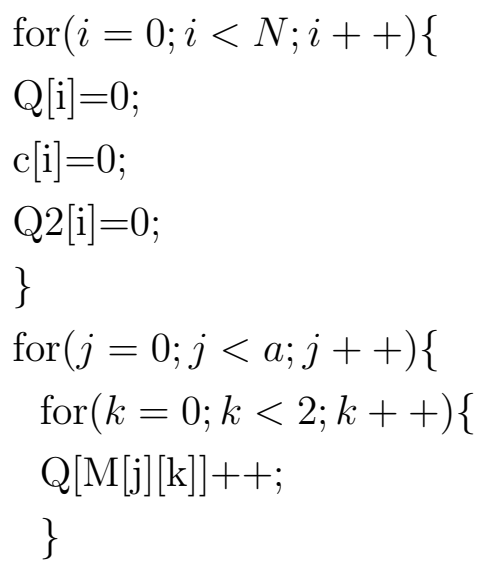


\}

$\mathrm{s}=0$;

for $(i=0 ; i<N ; i++)\{$

$\mathrm{S}=\mathrm{S}+\mathrm{Q}[\mathrm{i}]$;

\}

$\mathrm{km}=($ float $) \mathrm{s} /($ float $) \mathrm{N}$;

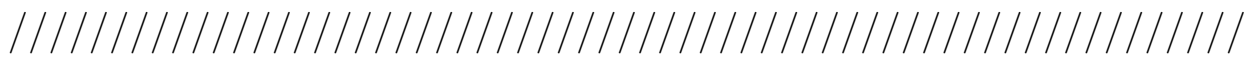

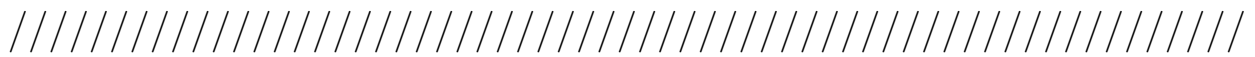

//Representação de vizinhos.

$$
\begin{aligned}
& \mathrm{A}=\left(\text { int }^{* *}\right) \operatorname{malloc}\left(\mathrm{N}^{*} \operatorname{sizeof}\left(\text { int }^{*}\right)\right) \text {; } \\
& \mathrm{B}=\left(\text { int }^{* *}\right) \operatorname{malloc}\left(\mathrm{N}^{*} \operatorname{sizeof}\left(\text { int }^{*}\right)\right) \text {; } \\
& \mathrm{C}=\left(\text { int } *^{*}\right) \operatorname{malloc}\left(\mathrm{N}^{*} \operatorname{sizeof}\left(\operatorname{int}^{*}\right)\right) \text {; } \\
& \text { for }(i=0 ; i<N ; i++)\{ \\
& \mathrm{A}[\mathrm{i}]=\left(\text { int }^{*}\right) \operatorname{malloc}\left(\mathrm{Q}[\mathrm{i}]{ }^{*} \text { sizeof }(\text { int })\right) \text {; } \\
& \text { \} } \\
& \text { for }(k=0 ; k<a ; k++)\{ \\
& \mathrm{i}=\mathrm{M}[\mathrm{k}][0] \text {; } \\
& \mathrm{j}=\mathrm{M}[\mathrm{k}][1] \text {; } \\
& \mathrm{A}[\mathrm{i}][\mathrm{c}[\mathrm{i}]++]=\mathrm{j} \text {; } \\
& \mathrm{A}[\mathrm{j}][\mathrm{c}[\mathrm{j}]++]=\mathrm{i} \text {; } \\
& \text { \} }
\end{aligned}
$$

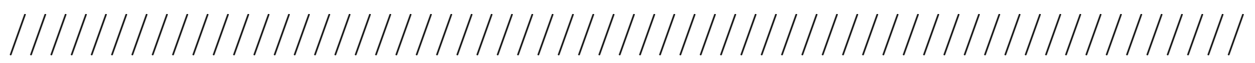

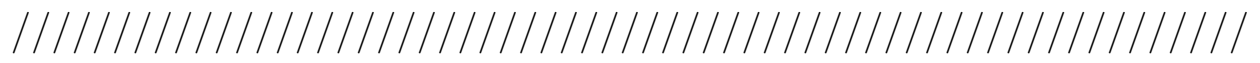

//Grau médio dos primeiros vizinhos. 
//Cálculo de $\langle k v\rangle$.

//sv3 será o grau médio dos primeiros vizinhos.

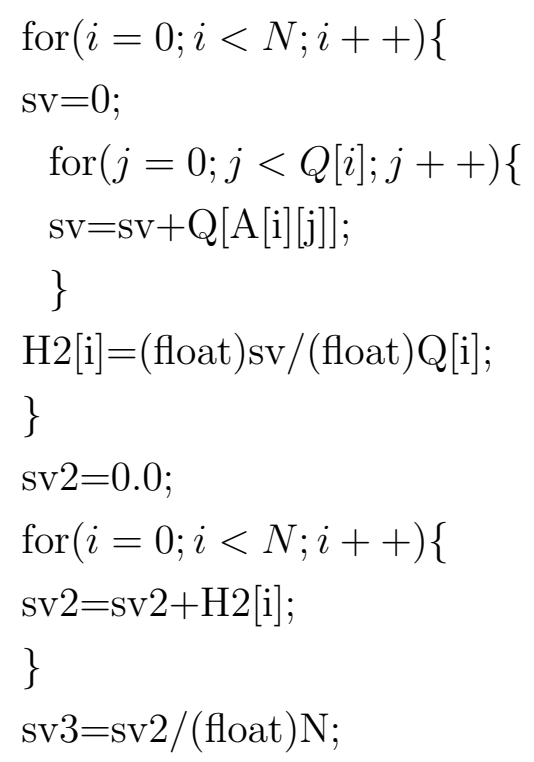

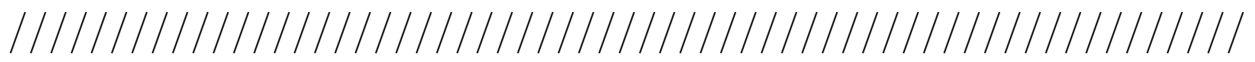

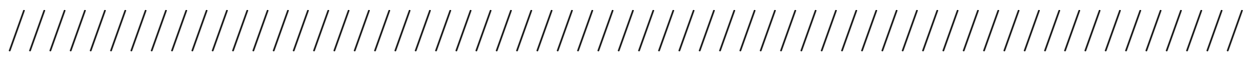

//Contabilizando os segundos vizinhos.

//F[i] será o número de segundos vizinhos do vértice i.

//Cálculo de $\left\langle k^{\prime}\right\rangle$.

$/ /\left\langle k^{\prime}\right\rangle$ será identificado como tm.

$$
\begin{aligned}
& \text { for }(i=0 ; i<N ; i++)\{ \\
& \mathrm{D}=0 ; \\
& \quad \text { for }(j=0 ; j<Q[i] ; j++)\{ \\
& \quad \text { for }(k=0 ; k<Q[A[i][j]] ; k++)\{ \\
& \quad \mathrm{h}=0 ; \\
& \quad \text { for }(l=0 ; l<Q[i] ; l++)\{
\end{aligned}
$$




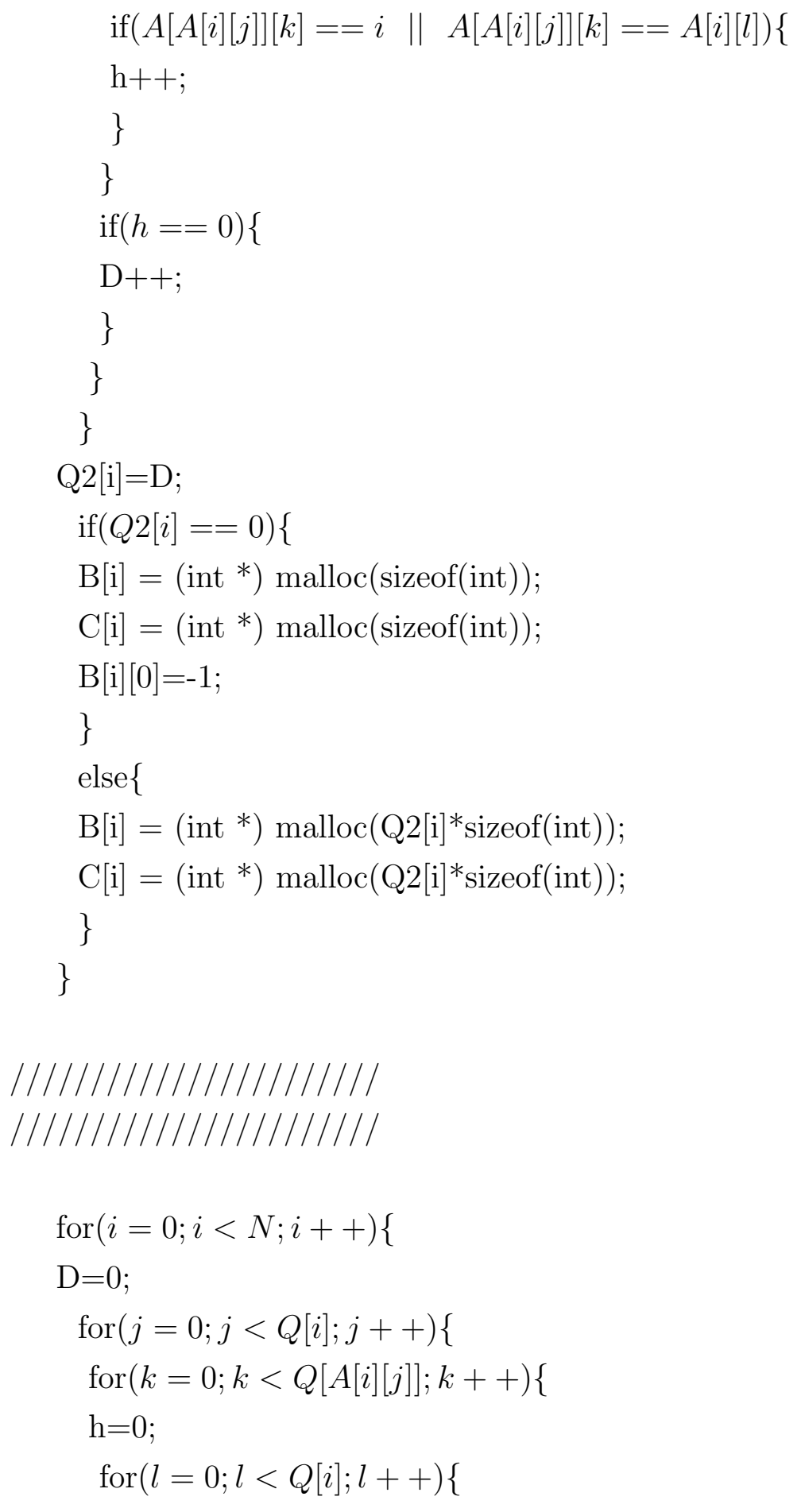

$$
\begin{aligned}
& \text { for }(i=0 ; i<N ; i++)\{ \\
& \mathrm{D}=0 ; \\
& \quad \text { for }(j=0 ; j<Q[i] ; j++)\{ \\
& \quad \text { for }(k=0 ; k<Q[A[i][j]] ; k++)\{ \\
& \quad \mathrm{h}=0 ; \\
& \quad \text { for }(l=0 ; l<Q[i] ; l++)\{
\end{aligned}
$$




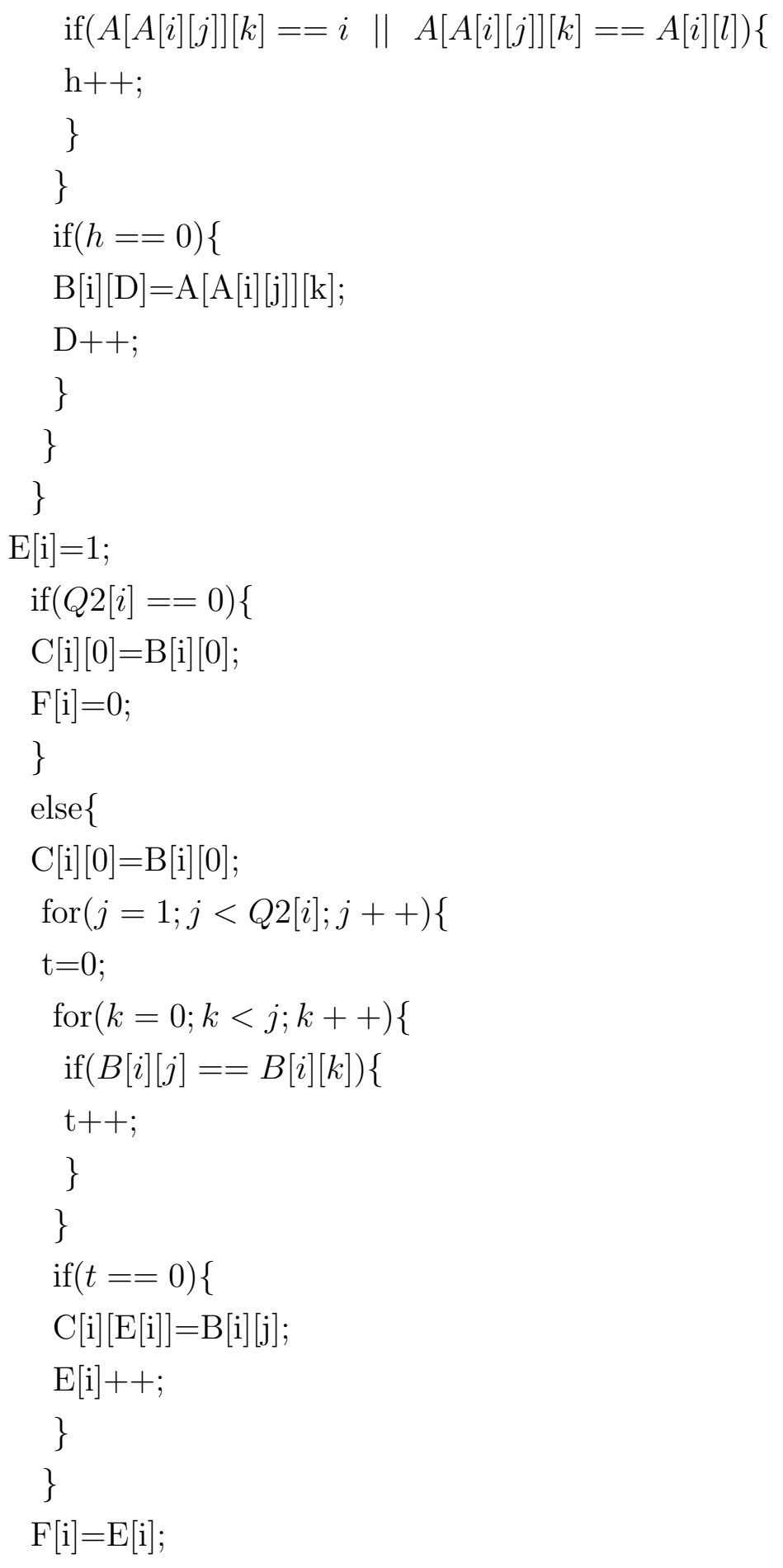




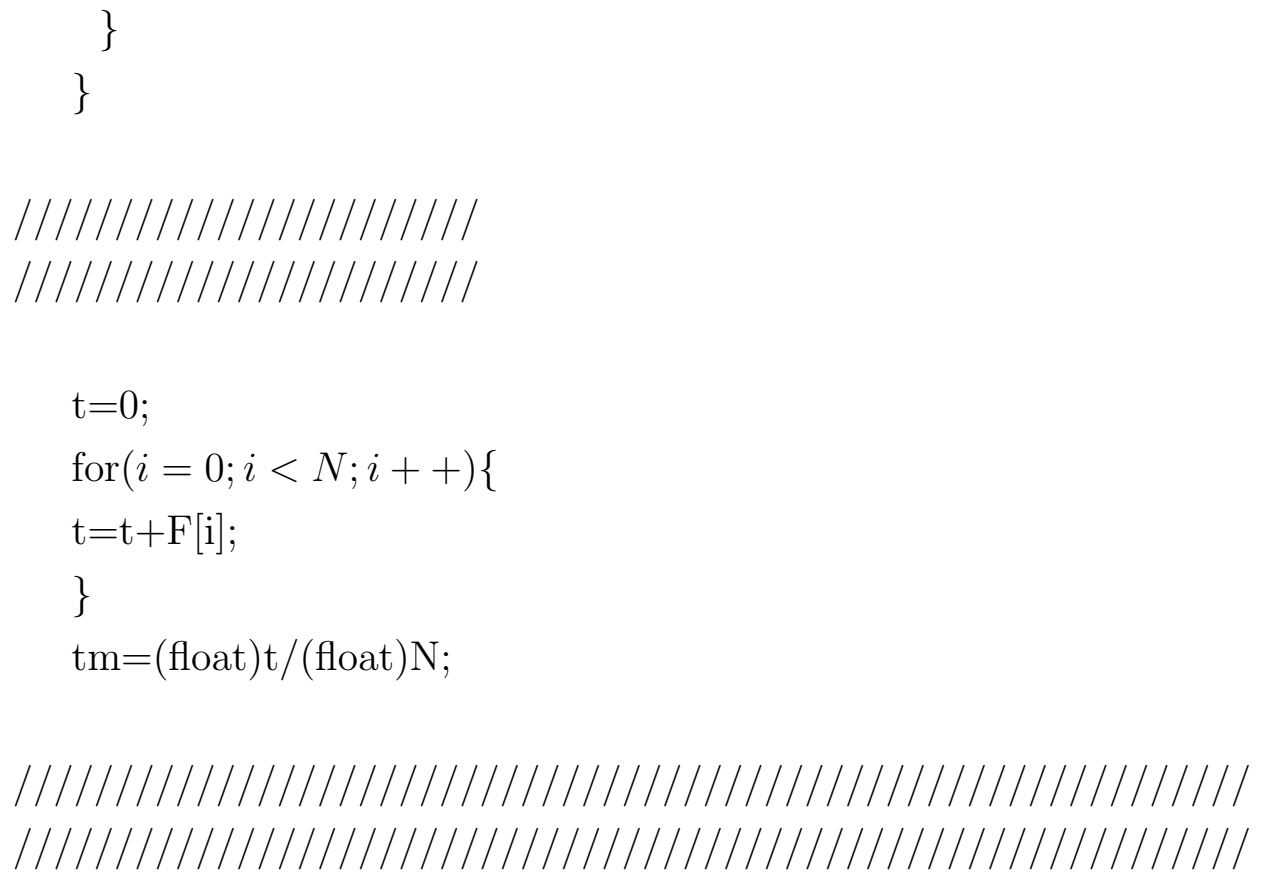

//Determinando a interseção entre a primeira e segunda vizinhança.

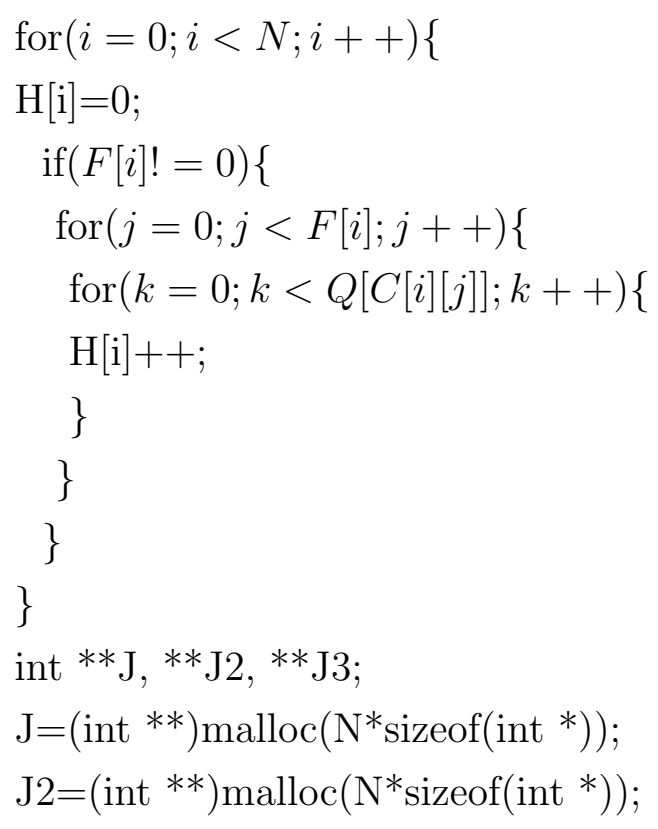




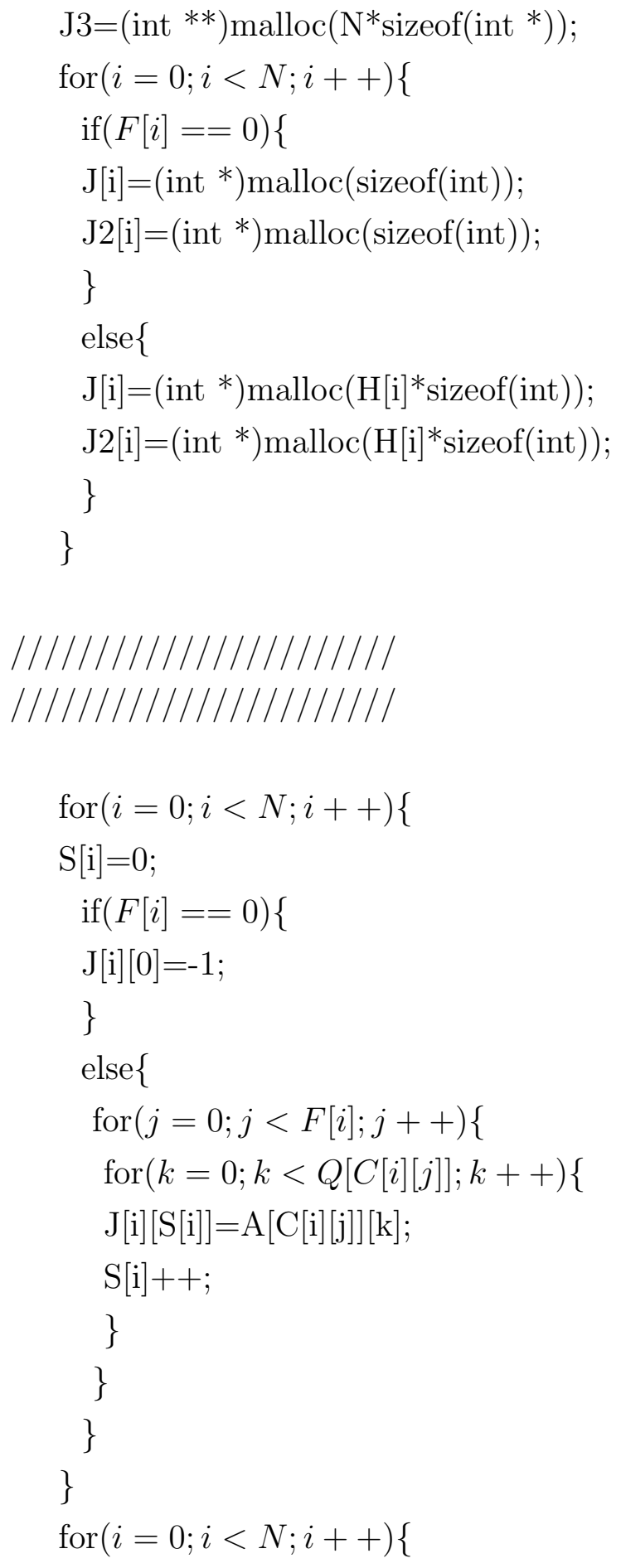




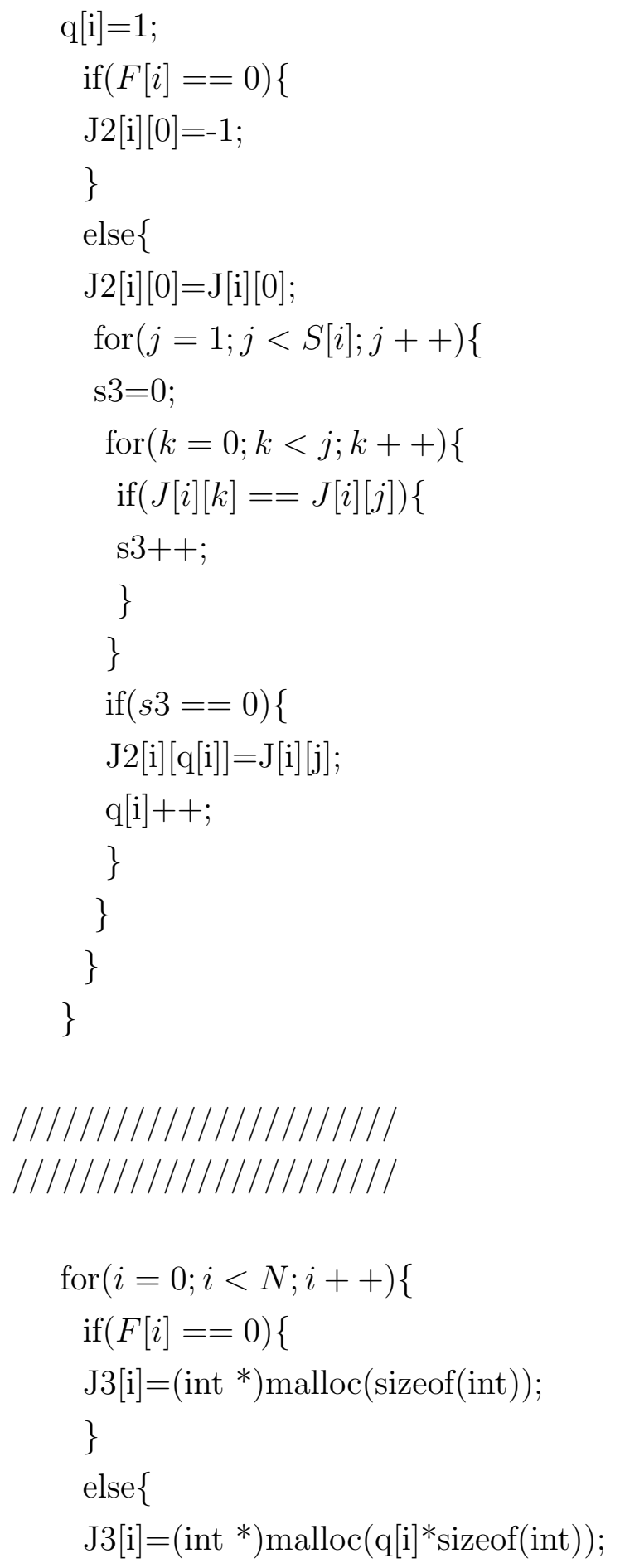




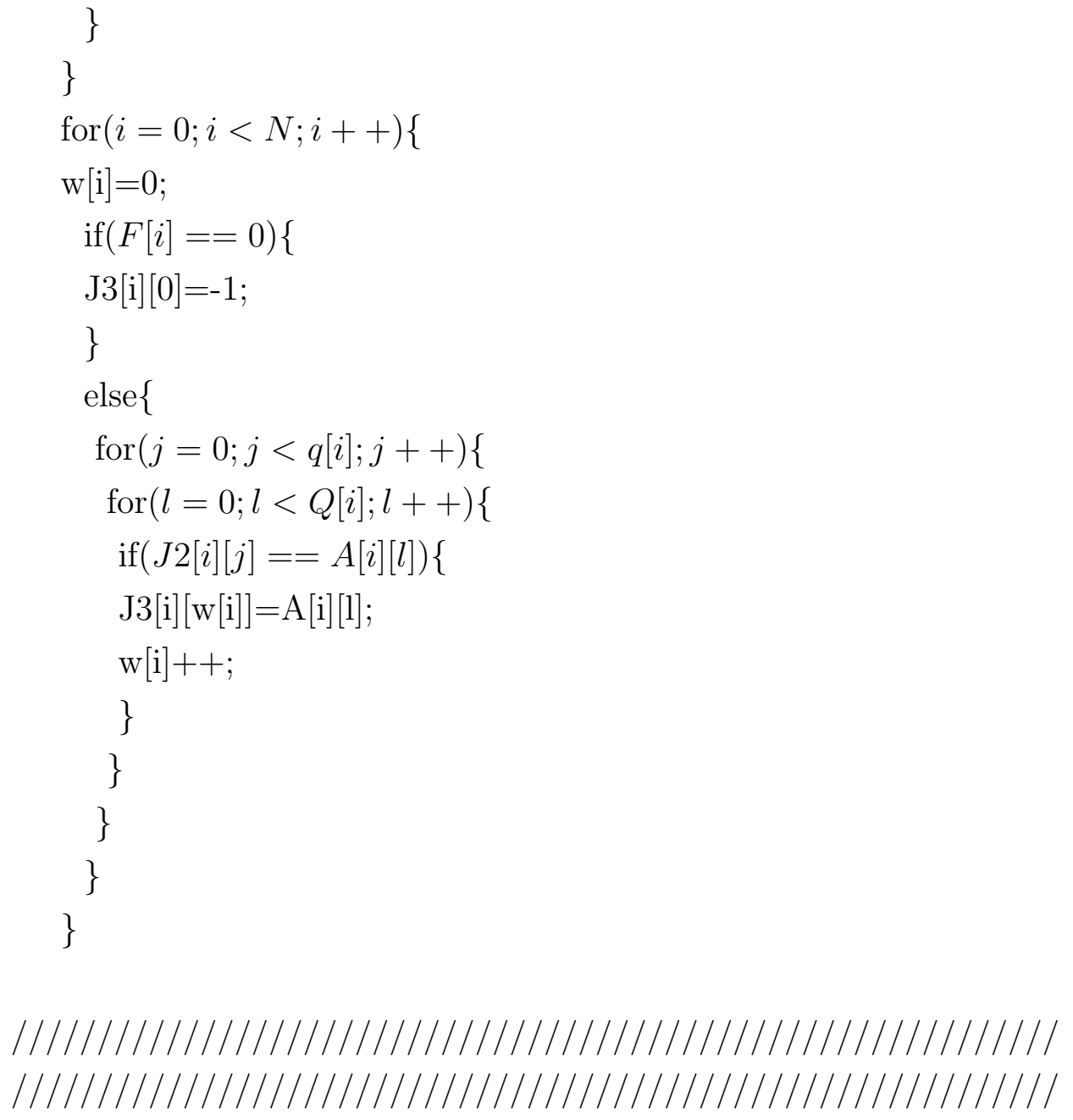

//Cálculo de $\langle l\rangle$.

$/ /\langle l\rangle$ é indetificado como sf.

float $\operatorname{lmm}[\mathrm{N}]$;

float ${ }^{* *} \mathrm{~lm}$;

int D100;

float sm, smm, sf;

$\operatorname{lm}=\left(\right.$ float $\left.{ }^{* *}\right) \operatorname{malloc}\left(\mathrm{N}^{*}\right.$ sizeof $($ float $\left.*)\right)$; 


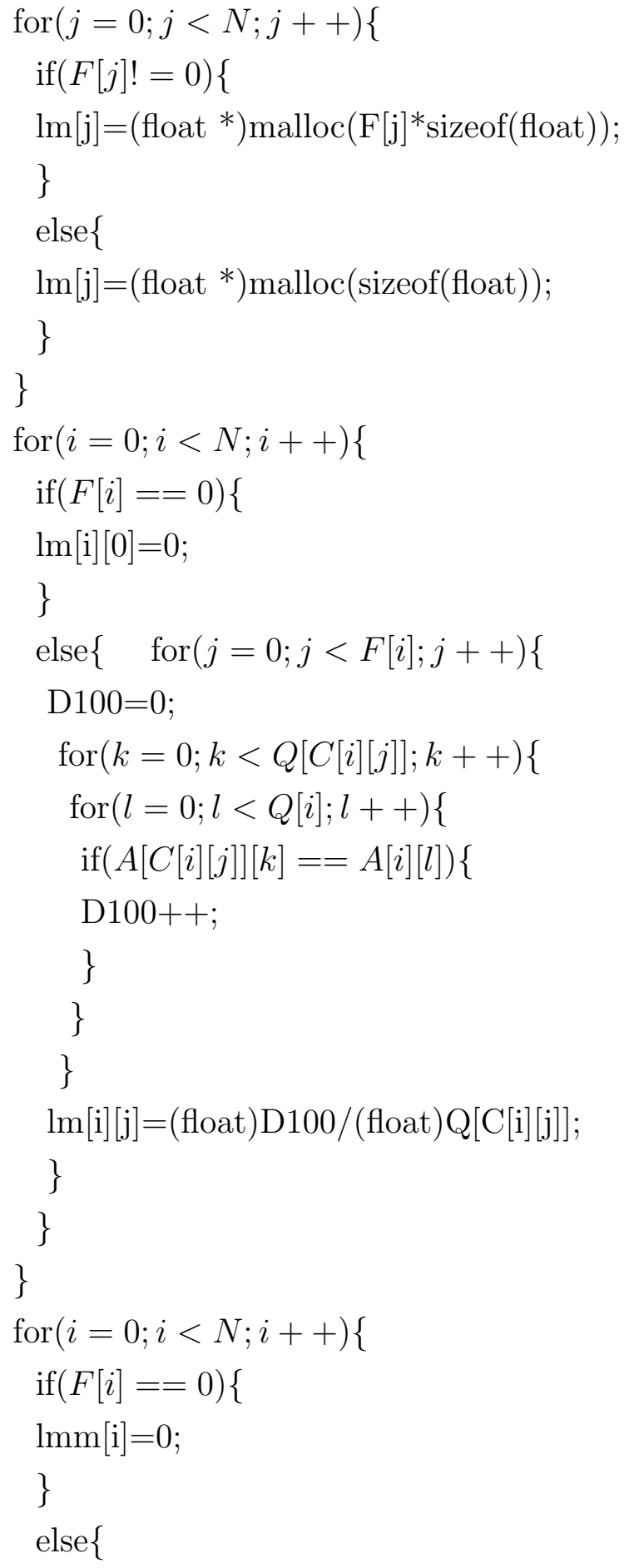




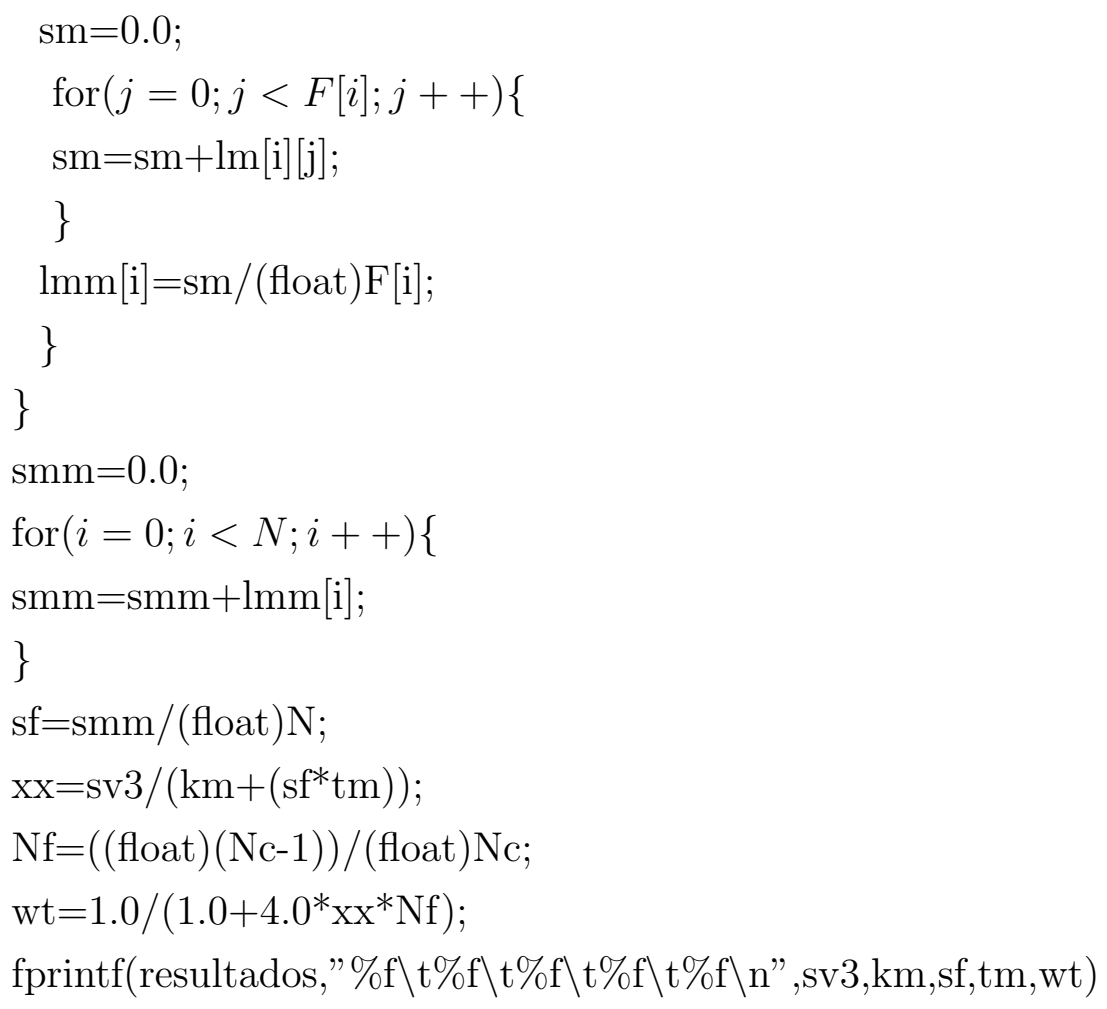

//Encerrando arquivos e ponteiros. 


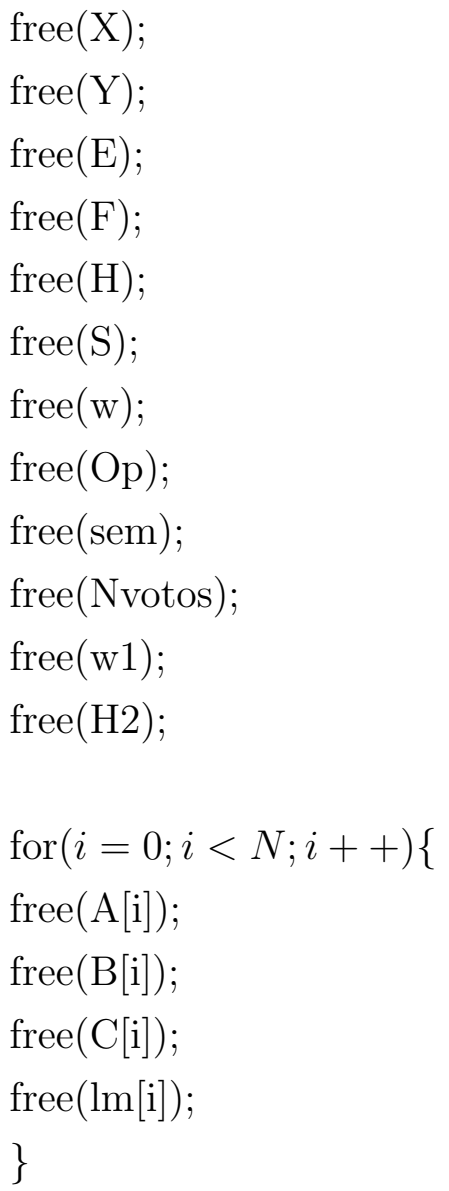




\section{Apêndice G}

\section{Algoritmo Utilizado para a Realização da Dinâmica do Modelo Sznajd Complexo}

Algoritmo de Monte Carlo utilizado para a realização da dinâmica do Modelo Sznajd Complexo em Linguagem C.

\#include $\langle$ stdio.h $\rangle$

\#include $\langle$ math.h $\rangle$

\#include $\langle$ stdlib.h $\rangle$

\#define noise 0.1

\#define TMC 100000

\#define Nc 10

\#define N 10000

\#define $\mathrm{m} 5$

\#define a $(\mathrm{m}+1) * \mathrm{~m} / 2+(\mathrm{N}-(\mathrm{m}+1))^{*} \mathrm{~m}$

//Modelo de Sznajd em uma rede BA com N vértices e conectividade mínima m. 
//Declaração de variáveis.

int main(int argc, char *argv[])\{

int ${ }^{*} \mathrm{M},{ }^{*} \mathrm{Q},{ }^{*} \mathrm{q},{ }^{*} \mathrm{Q} 2,{ }^{*} \mathrm{c},{ }^{*} \mathrm{P},{ }^{*} \mathrm{X},{ }^{*} \mathrm{Y},{ }^{*} \mathrm{E},{ }^{*} \mathrm{~F},{ }^{*} \mathrm{H}, *{ }^{*}, *{ }_{\mathrm{w}},{ }^{*} \mathrm{Op}$, *sem, *Nvotos;

int b, g, G, d, f, i, r, s, t, u, j, k, l, p, z, D, Z, h, n, v, maior, x, s3, r0, r1, dsem;

int sv, sort0, sort1, sort2, sort3;

float W1, t1, sv2, sv3, wsort;

float ${ }^{*} \mathrm{w} 1,{ }^{*} \mathrm{H} 2$;

int ${ }^{* *} \mathrm{~A},{ }^{* *} \mathrm{C}$;

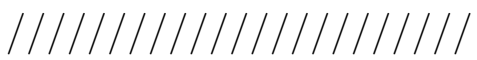

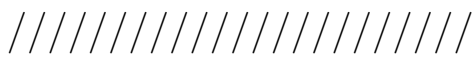

$\mathrm{M}=\left(\right.$ int $\left.^{* *}\right) \operatorname{malloc}\left(\mathrm{a}^{*} \operatorname{sizeof}\left(\right.\right.$ int $\left.\left.^{*}\right)\right)$;

for $(i=0 ; i<a ; i++)\{$

$\mathrm{M}[\mathrm{i}]=($ int $*) \operatorname{malloc}(2 * \operatorname{sizeof}($ int $))$;

\}

$\mathrm{Q}=($ int $*) \operatorname{malloc}\left(\mathrm{N}^{*} \operatorname{sizeof}(\mathrm{int})\right)$;

$\mathrm{q}=($ int $*) \operatorname{malloc}\left(\mathrm{N}^{*}\right.$ sizeof(int $)$ );

Q2=(int *)malloc $\left(\mathrm{N}^{*}\right.$ sizeof(int $)$ );

$\mathrm{c}=($ int $*) \operatorname{malloc}\left(\mathrm{N}^{*}\right.$ sizeof(int $)$; 


$$
\begin{aligned}
& \mathrm{P}=\left(\text { int }^{*}\right) \operatorname{malloc}\left(\mathrm{N}^{*} \operatorname{sizeof}(\text { int })\right) \text {; } \\
& \mathrm{X}=\left(\text { int }^{*}\right) \operatorname{malloc}\left(\mathrm{m}^{*} \text { sizeof }(\text { int })\right) \text {; } \\
& \mathrm{Y}=(\text { int } *) \operatorname{malloc}\left(\mathrm{m}^{*} \operatorname{sizeof}(\text { int })\right) \text {; } \\
& \mathrm{E}=\left(\text { int }^{*}\right) \operatorname{malloc}\left(\mathrm{N}^{*} \operatorname{sizeof}(\text { int })\right) \text {; } \\
& \mathrm{F}=(\text { int } *) \operatorname{malloc}\left(\mathrm{N}^{*} \operatorname{sizeof}(\text { int })\right) \text {; } \\
& \mathrm{H}=(\text { int } *) \operatorname{malloc}\left(\mathrm{N}^{*} \text { sizeof }(\text { int })\right) \text {; } \\
& \mathrm{S}=(\text { int } *) \operatorname{malloc}\left(\mathrm{N}^{*} \operatorname{sizeof}(\text { int })\right) \text {; } \\
& \mathrm{w}=\left(\text { int }^{*}\right) \operatorname{malloc}\left(\mathrm{N}^{*} \text { sizeof }(\text { int })\right) \text {; } \\
& \mathrm{Op}=(\text { int } *) \operatorname{malloc}\left(\mathrm{N}^{*} \operatorname{sizeof}(\text { int })\right) \text {; } \\
& \text { sem } \left.=(\text { int } *) \text { malloc }\left(\mathrm{Nc}^{*} \text { sizeof(int }\right)\right) \text {; } \\
& \text { Nvotos }=(\text { int } *) \operatorname{malloc}((\mathrm{Nc}+1) * \operatorname{sizeof}(\mathrm{int})) \text {; } \\
& \mathrm{w} 1=(\text { float } *) \operatorname{malloc}\left(\mathrm{N}^{*} \text { sizeof(float }\right) \text {; } \\
& \left.\mathrm{H} 2=\left(\text { float }{ }^{*}\right) \operatorname{malloc}\left(\mathrm{N}^{*} \text { sizeof(float }\right)\right) \text {; }
\end{aligned}
$$

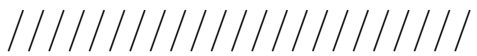

$$
\begin{aligned}
& 1 / / / / / / / / / / / / / / / / / / 1
\end{aligned}
$$

FILE *frames_com_ruido;

FILE *numero_votos_com_ruido;

frames_com_ruido=fopen("frames_com_ruido_0_1.dat","w");

numero_votos_com_ruido=fopen("numero_votos_com_ruido_0_1.dat", "w");

$\mathrm{f}=0$;

$\mathrm{g}=0$;

$\operatorname{srand}(\operatorname{atoi}(\operatorname{argv}[1]))$ 
//Configuração inicial da rede.

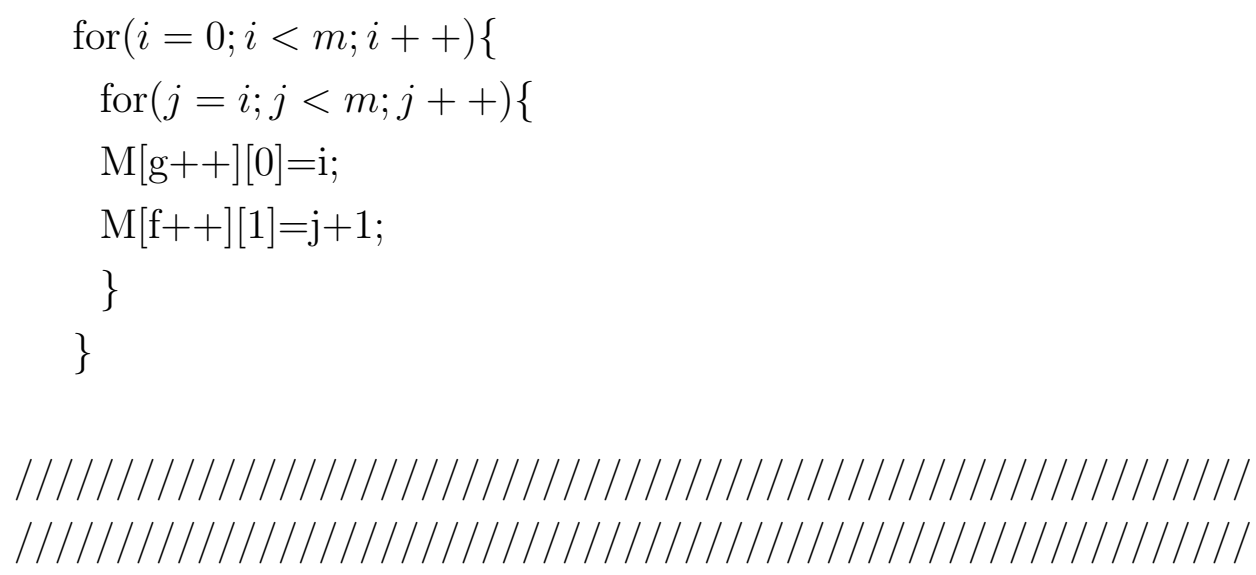

//Construindo a rede.

$\mathrm{z}=\mathrm{m}+1 ;$

for $(i=m *(m+1) / 2 ; i<=m *(m+1) / 2+m *(N-m-2) ; i=i+m)\{$ $\mathrm{r}=\operatorname{rand}() \% \mathrm{i}$;

$\mathrm{s}=\operatorname{rand}() \% 2$;

$\mathrm{X}[0]=\mathrm{M}[\mathrm{r}][\mathrm{s}]$;

$\mathrm{Y}[0]=\mathrm{M}[\mathrm{r}][\mathrm{s}]$;

$\mathrm{M}[\mathrm{i}][0]=\mathrm{z}++$;

$\mathrm{M}[\mathrm{i}][1]=\mathrm{Y}[0]$;

for $(j=1 ; j<m ;)\{$

$\mathrm{t}=\operatorname{rand}() \% \mathrm{i}$;

$\mathrm{u}=\operatorname{rand}() \% 2$;

$\mathrm{X}[\mathrm{j}]=\mathrm{M}[\mathrm{t}][\mathrm{u}] ;$ 


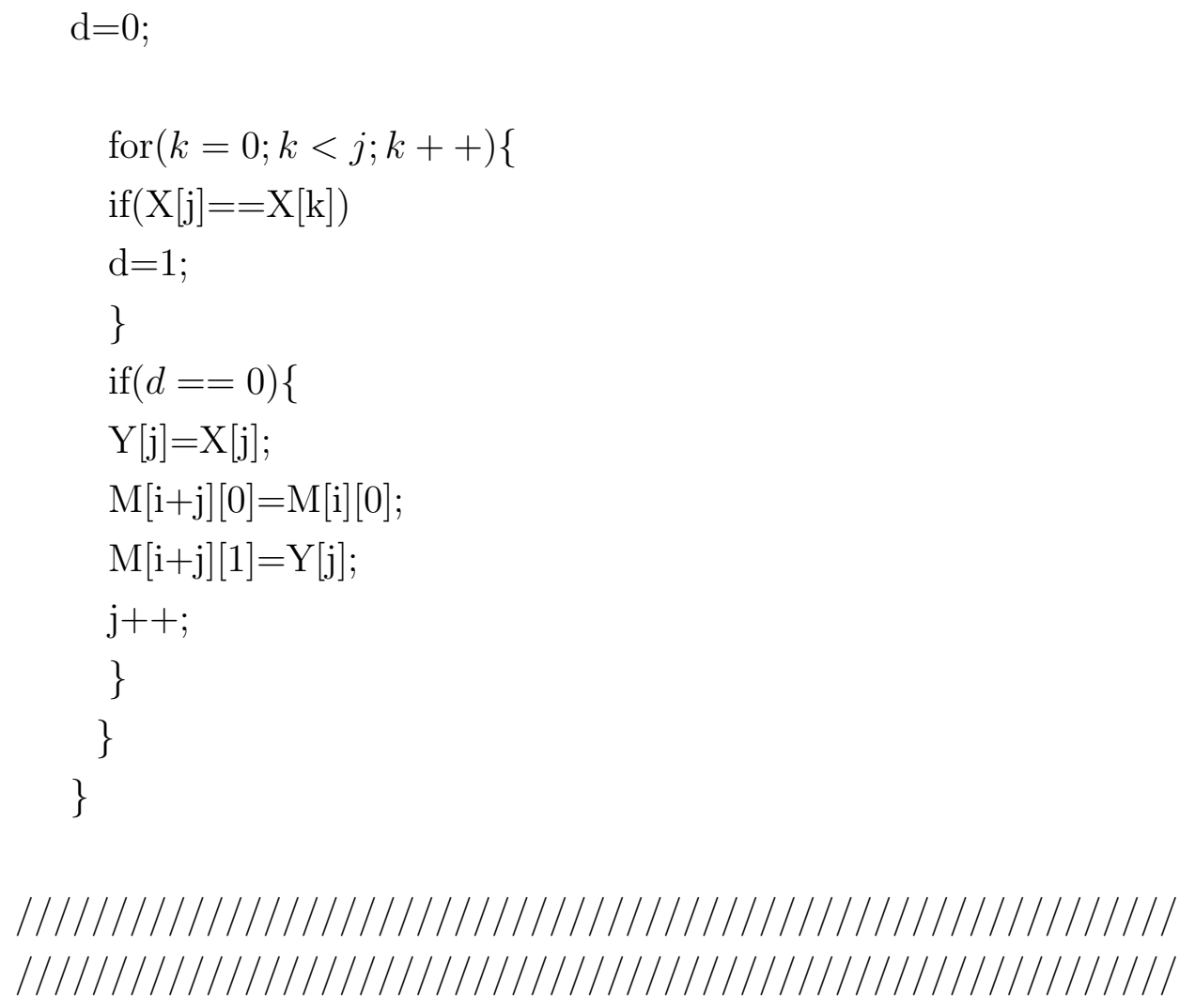

//Contabilizando o grau dos vértices.

$/ / Q[i]$ é o grau do vértice i.

$$
\begin{aligned}
& \text { for }(i=0 ; i<N ; i++)\{ \\
& \text { Q }[\mathrm{i}]=0 ; \\
& \mathrm{c}[\mathrm{i}]=0 ; \\
& \mathrm{Q} 2[\mathrm{i}]=0 ; \\
& \} \\
& \text { for }(j=0 ; j<a ; j++)\{ \\
& \quad \text { for }(k=0 ; k<2 ; k++)\{ \\
& \quad \mathrm{Q}[\mathrm{M}[\mathrm{j}][\mathrm{k}]]++;
\end{aligned}
$$




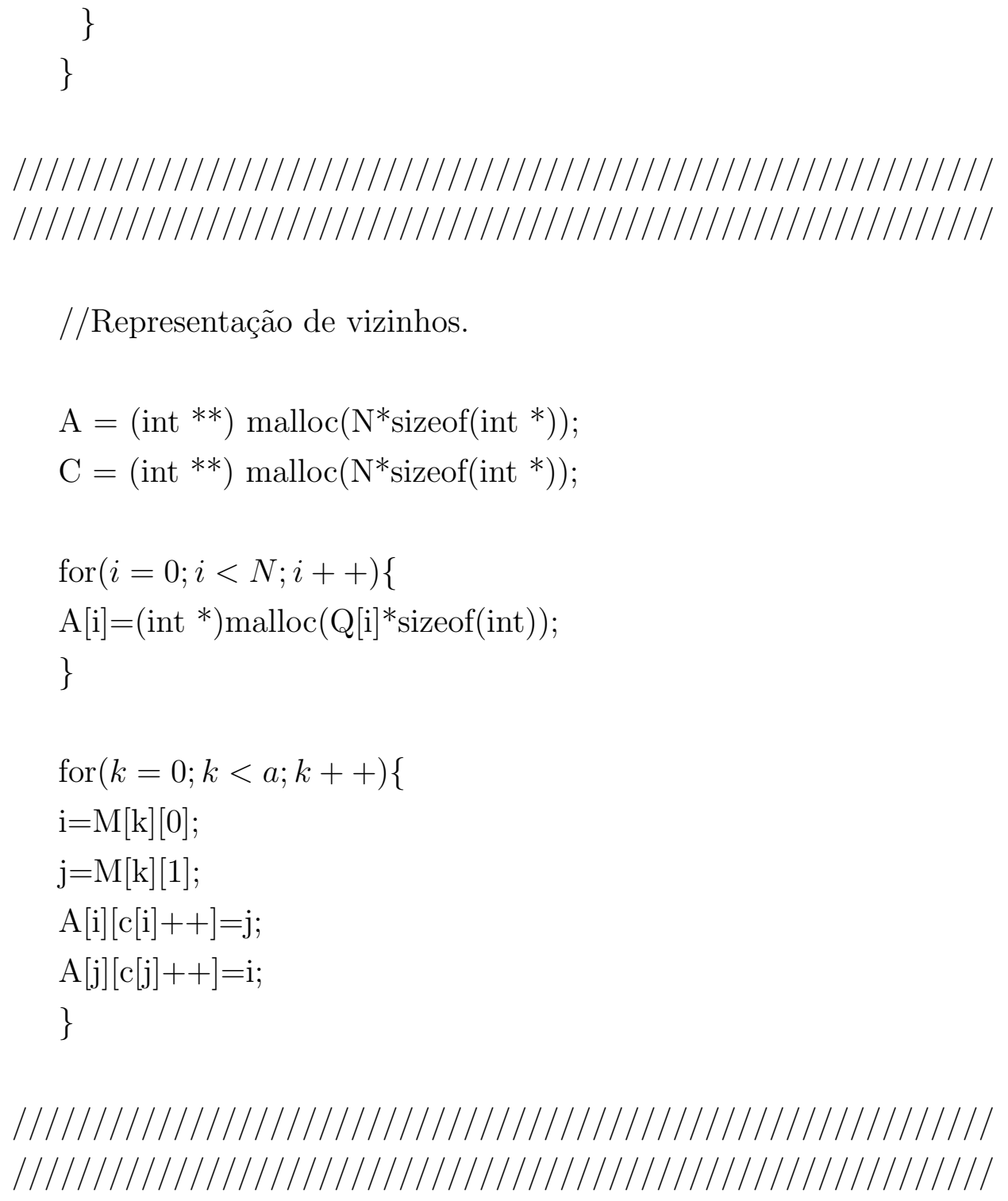

//Contabilizando os segundos vizinhos.

//F[i] será o número de segundos vizinhos do vértice i.

for $(i=0 ; i<N ; i++)\{$

$\mathrm{D}=0$; 


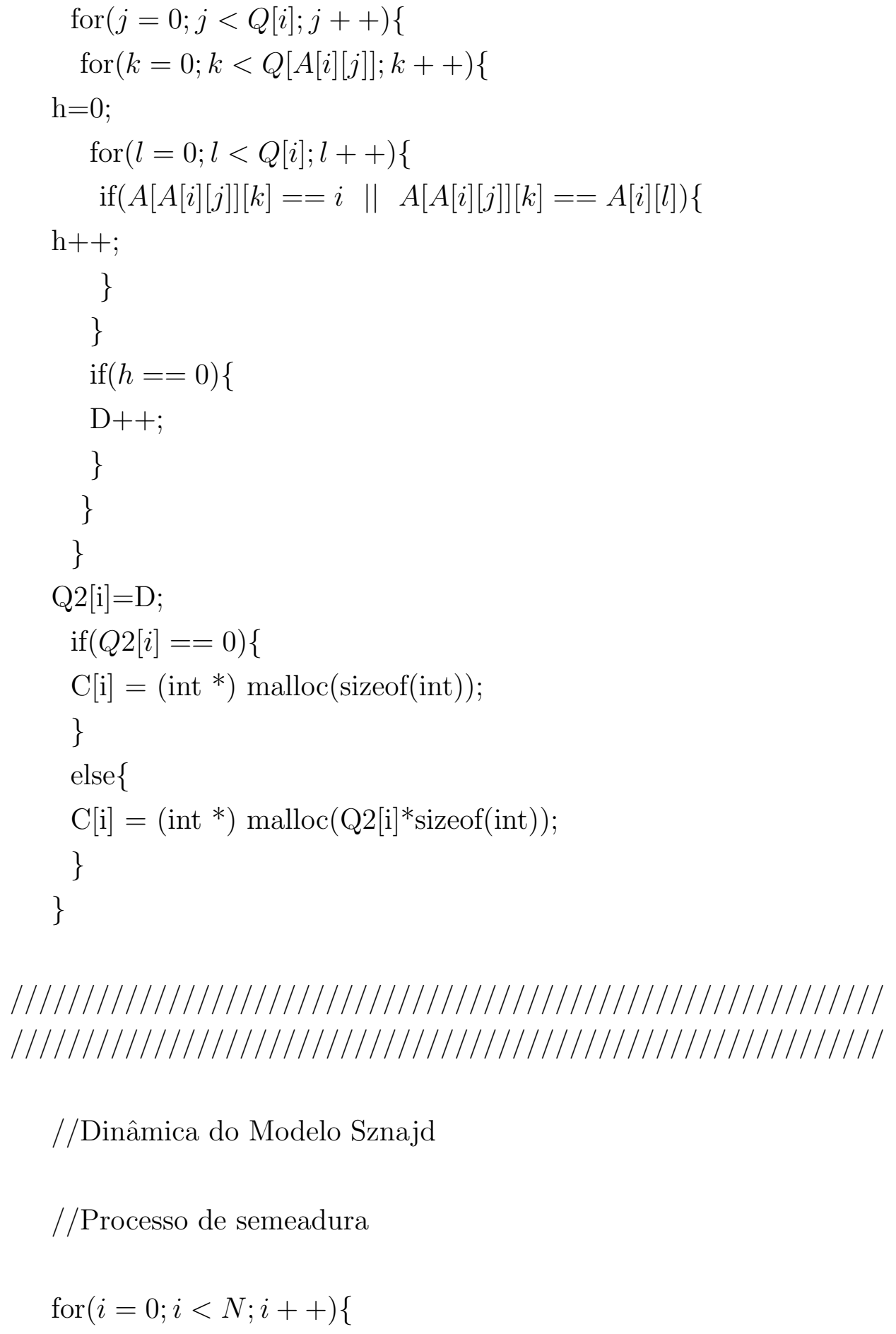

//Dinâmica do Modelo Sznajd

//Processo de semeadura

for $(i=0 ; i<N ; i++)\{$ 


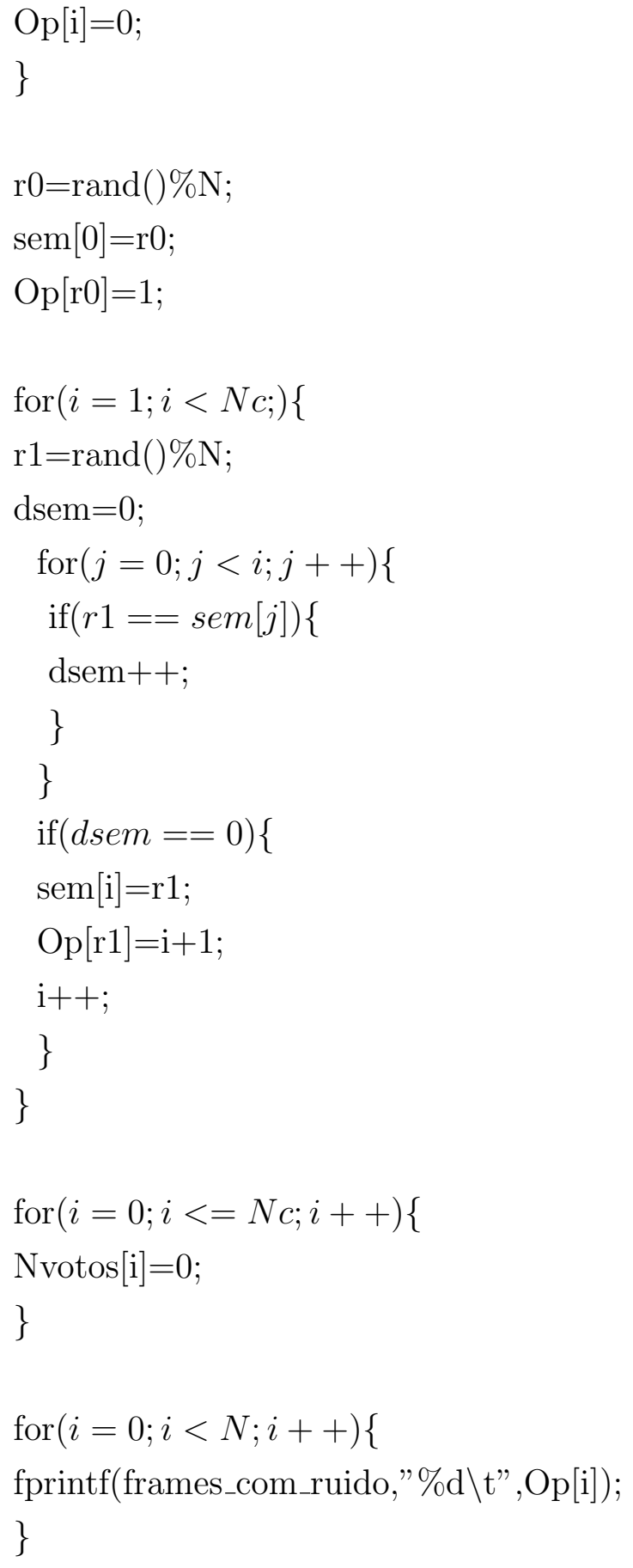




\section{fprintf(frames_com_ruido," \n");}

fprintf(numero_votos_com_ruido,"\% $/ \mathrm{d}$ t",0);

$$
\begin{aligned}
& \text { for }(i=0 ; i<=N c ; i++)\{ \\
& \quad \operatorname{for}(j=0 ; j<N ; j++)\{ \\
& \quad \text { if }(i==O p[j])\{
\end{aligned}
$$$$
\text { Nvotos }[\mathrm{i}]++ \text {; }
$$$$
\text { \} }
$$

\}

fprintf(numero_votos_com_ruido," \% d tt",Nvotos[i] );

\}

fprintf(numero_votos_com_ruido," \n");

l/////////////////I

$/ / / / / / / / / / / / / / / / / / 1$

//Executando a dinâmica

$$
\begin{aligned}
& \text { for }(t=0 ; t<=T M C ; t++)\{ \\
& \text { for }(p=0 ; p<N ; p++)\{ \\
& \text { sort } 0=\operatorname{rand}() \% \mathrm{~N} ; \\
& \text { wsort }=(\text { float }) \text { rand }() /(\text { float }) \text { RAND_MAX; } \\
& \text { if }(\text { wsort }<=\text { noise })\{ \\
& \text { sort } 1=(\operatorname{rand}() \% \mathrm{Nc})+1 ; \\
& \text { Op }[\operatorname{sort} 0]=\operatorname{sort} 1 ; \\
& \} \\
& \text { else }\{ \\
& \text { if }(O p[\text { sort } 0]==0)\{ \\
& \quad / / \text { Nada ocorre. }
\end{aligned}
$$




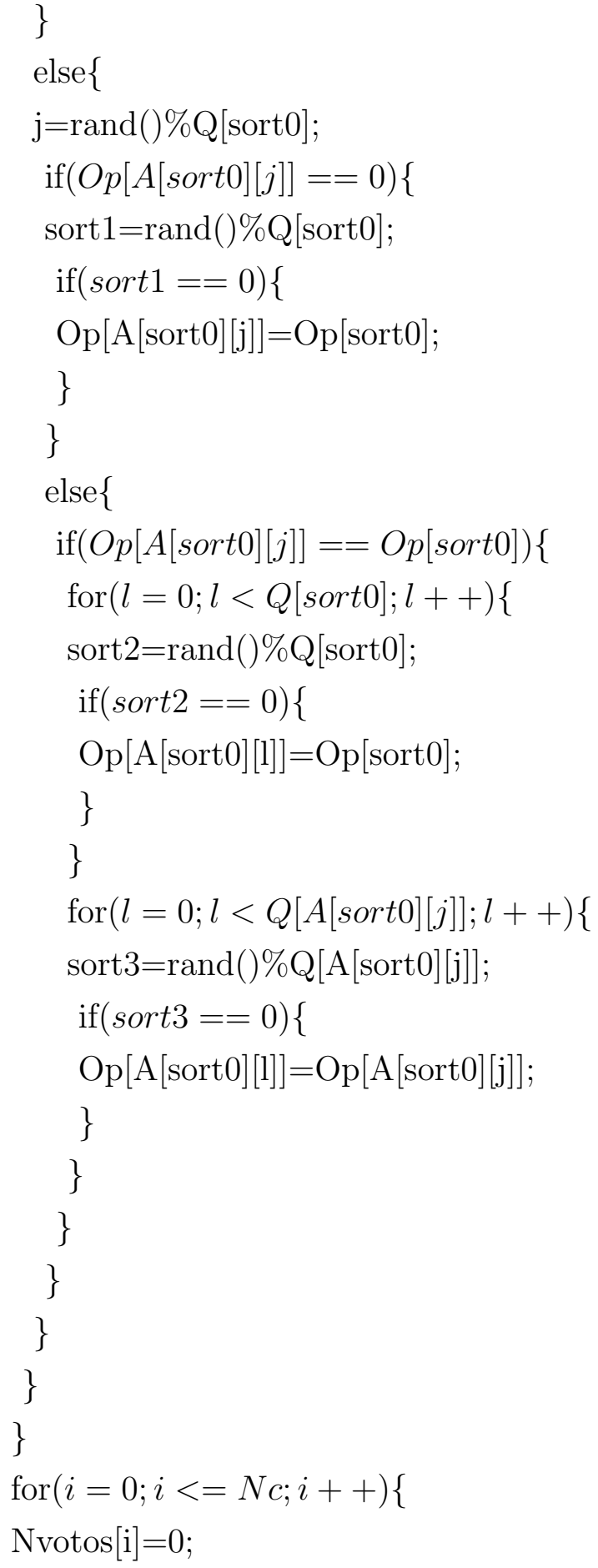




\section{\}}

for $(i=0 ; i<N ; i++)\{$

fprintf(frames_com_ruido,"\% $/ \mathrm{d} \backslash \mathrm{t}$, Op $[\mathrm{i}]$ );

\}

fprintf(frames_com_ruido," \n");

fprintf(numero_votos_com_ruido,"\% \% $\backslash \mathrm{t}$, t)

$$
\begin{aligned}
& \text { for }(i=0 ; i<=N c ; i++)\{ \\
& \operatorname{for}(j=0 ; j<N ; j++)\{ \\
& \quad \operatorname{if}(i==O p[j])\{
\end{aligned}
$$

Nvotos $[\mathrm{i}]++$;

\}

\}

fprintf(numero_votos_com_ruido," \%d $\backslash$ t" ,Nvotos[i] );

\}

fprintf(numero_votos_com_ruido," \n");

\}

//Encerrando arquivos e ponteiros.
free $(\mathrm{M}[\mathrm{i}])$;
\}
free $(\mathrm{Q})$;
free(q);
free(Q2);
free (c);
free(P);

for $(i=0 ; i<a ; i++)\{$ 


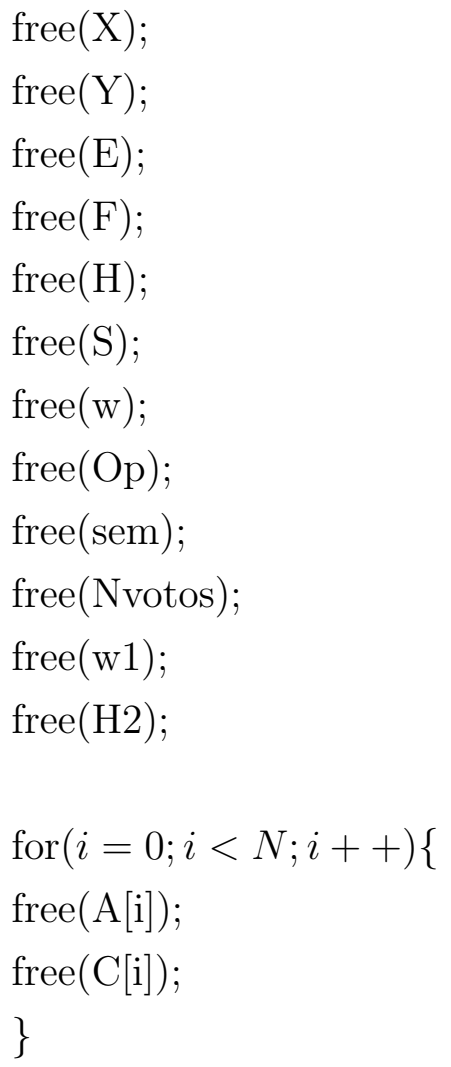

return 0 ;

\} 


\section{Referências Bibliográficas}

[1] K. Sznajd-Weron, J. Sznajd. Opinion Evolution in Closed Community. Int. J. Mod. Phys. C, 11: 1157-1165, 2000.

[2] C. Castellano, S. Fortunato, V. Loreto. Statistical Physics of Social Dynamics. Rev. Mod. Phys., v. 81: 591, 2009.

[3] R. N. Costa-Filho, M. P. Almeida, J. S. Andrade Jr., J. E. Moreira. Scaling Behaviour in a Proportional Voting Process. Phys. Rev. E, v. 60: 1067-1068, 1999.

[4] A. Timpanaro, C. P. C. Prado. Generalized Sznajd Model for Opinion Propagation. Phys. Rev. E, v. 80:21119, 2009.

[5] A. T. Bernardes, D. Stauffer, J. Kertész. Election Results and the Sznajd model on Barabasi Network. Eur. Phys. J. B, v. 25:123$127,2002$.

[6] F. S. Vannucchi. O Modelo de Sznajd em Redes Complexas. Dissertação de Mestrado, Instituto de Física, Universidade de São Paulo, 2006.

[7] R. Albert, A.-L. Barabási. Statistical Mechanics of Complex Networks. Review of Modern Physics, 74: 47, 2002.

[8] M. E. J. Newman. The Structure and Function of Complex Networks. SIAM Review, 45: 167-256, 2003. 
[9] A. S. Klovdahl, J. J. Potterat, D. E. Woodhouse, J. B. Muth, S. Q. Muth, W. W. Darrow,. Social Networks and Infectious Disease: The Colorado Springs Study. Social Science and Medicine, 38: 79, 1994.

[10] T. P. Peixoto, C. P. C. Prado. Criticalidade Auto-Organizada no Modelo Olami-Feder-Christensen para Terremotos: Dinâmica de Epicentros. Comunicação Pessoal, 2006.

[11] Wikipédia, a enciclopédia livre. Teoria dos Grafos. Disponível em http: //pt.wikipedia.org/wiki/Teoria_dos_grafos.

[12] P. Erdös, A. Rényi. On Random Graphs. Publ. Math. Debrecen, 6:290, 1959 .

[13] S. Milgram. The Small World Problem. Psycology Today, 2:60-67, 1967.

[14] G. U. Yule. A Mathematical Theory of Evolution Based on the Conclusions of Dr. J. C. Willis. Philos. Trans. R. Soc. London B, 213:21, 1925.

[15] D. J. S. Price. A General Theory of Bibliometric and Other Cumulative Advantage Processes. J. Amer. Soc. Inform. Sci., 27:292, 1976.

[16] R. Albert, A.-L. Barabási. Emergence of Scaling in Random Networks. Science, 286:506, 1999.

[17] M. C. Gonzales, A. O. Souza, H. J. Herrmann. Oppinion Formation on a Deterministic Pseudo Fractal Network. Int. J. Mod. Phys. C, v. 12: 45-57, 2004.

[18] T. Tomé, M. J. Oliveira. Dinâmica Estocástica e Irreversibilidade. Edusp, São Paulo, 2001. 
[19] S. R. A. Salinas. Introdução à Mecânica Estatística. Edusp, São Paulo, 1997.

[20] K. Sznajd-Weron,. Sznajd Model and its Applications. Acta Phys. Pol. B, 36: 2537-2547, 2005.

[21] D. Stauffer, A. O. Sousa, S. M. Oliveira. Generalization to Square Lattice of Sznajd Sociophysics Model. Int. J. Mod. Phys., v. 11: 1239-1245, 2000.

[22] C. Schulze. Advertising in the Sznajd Marketing Model. Int. J. Mod. Phys. C, v. 14: 95-98, 2003.

[23] K. Sznajd-Weron, R. Weron. How Effective is Edvertising in Duopoly Markets? Physica A, v. 324: 437, 2003.

[24] K. Sznajd-Weron, R. Weron. A Simple Model of Price Formation. Int. J. Mod. Phys. C, v. 13: 115, 2002.

[25] A. T. Bernardes, U. M. S. Costa, A. D. Araújo, D. Stauffer. Damage Spreading, Coarsening Dynamics and Distribution of Political Votes in Sznajd Model on a Square Lattice. Int. J. Mod. Phys. C, v. 12: 159-167, 2001.

[26] J. R. Sanchez. A Modified One-Dimensional Sznajd Model. condmat/0408518.

[27] L. Behera, F. Schweitzer. On Spatial Consensus Formation: Is the Sznajd Model Different from a Voter Model? Int. J. Mod. Phys. $C$, v. $14: 1331,2003$.

[28] F. Slanina, H. Lavicka. Analytical Results for the Sznajd Model of Opinion Formation. Eur. Phys. J. B, v. 35: 279-288, 2003. 
[29] M. F. He, B. Li, L. D. Luo. Sznajd Model with "Social Temperature" and Defender on Small-World Networks. Int. J. Mod. Phys. C, 15: 997, 2004.

[30] F. S. Vannucchi, C. P. C. Prado. Sznadj Model and Proportional Elections: The Role of the Topology of the Network. Int. J. Mod. Phys. C, v. 20: 979-990, 2009.

[31] G. Deffuant, D. Neau, F. Amblard, G. Weisbuch. Mixing Beliefs Among Interacting Agents. Adv. Complex Syst., v. 3: 87-98, 2001.

[32] N. Fiedler-Ferrara, C. P. C. Prado. Caos Uma Introducao. $2^{a}$ ed. Edgar Blucher Ltda, São Paulo, 1994.

[33] E. L. Lima. Álgebra Linear. Coleção Matemática Universitária, IMPA, Rio de Janeiro, 2004.

[34] L. H. A. Monteiro. Sistemas Dinâmicos. Editora Livraria da Física, São Paulo, 2006.

[35] E. L. Lima. Análise Real. Coleção Matemática Universitária, IMPA, Rio de Janeiro, 2004.

[36] J. Marro, R. Dickmann. Nonequilibrium Phase Transitions on Lattices Models. Cambridge University Press, 1995. 